Alex Jader Sant'Ana

\title{
Avaliação da responsividade cardiovascular por meio da variação de pulso com diferentes fluídos em modelo experimental de hemodiluição normovolêmica aguda
}

Tese apresentada à Faculdade de Medicina de São Paulo para obtenção do título de Doutor em Ciências Área de concentração: Anestesiologia Orientador: Prof. Dr. José Otávio Costa Auler Junior 


\section{Dados Internacionais de Catalogação na Publicação (CIP)}

Preparada pela Biblioteca da

Faculdade de Medicina da Universidade de São Paulo

Creprodução autorizada pelo autor

Sant'Ana, Alex Jader

Avaliação da responsividade cardiovascular por meio da variação de pulso com diferentes fluídos em modelo experimental de hemodiluiçao normovolêmica aguda / Alex Jader Sant'Ana. -- São Paulo, 2009.

Tese(doutorado)--Faculdade de Medicina da Universidade de São Paulo. Departamento de Cirurgia.

Área de concentração: Anestesiologia.

Orientador: José Otávio Costa Auler Junior.

Descritores: 1.Pulso arterial 2.Hemodiluição 3.Solução salina 4.Amido 5.Suínos

USP/FM/SBD-124/09 
Dedico este trabalho:

Aos meus pais, Osmar e Maria, pelo amor, carinho e compreensão em todas as fases da minha vida. Por me ensinarem a viver!

Aos meus queridos irmãos Thiago e Guilherme.

À Thelma pela paciência, amor e por estar ao meu lado em todos os momentos! 
Ao Prof. Dr. José Otávio Costa Auler Junior, pela orientação deste estudo, pelo entusiasmo, apoio e confiança em meu trabalho.

À Profa. Dra. Denise Tabacchi Fantoni, por me ter aceitado e dado a oportunidade de fazer parte de sua equipe de pesquisa e trabalho na anestesiologia!

Ao Prof. Dr. Aury Nunes de Moraes, meu pai anestesiológico! Por me ter "adotado" e acreditado no meu trabalho.

Aos Amigos e companheiros de pós graduação por fazerem parte dessa fase de estudo e trabalho!

Ao Dr. César Dias Freire, pelo companheirismo nos anos em São Paulo, pelas conversas científicas e não científicas!

Aos amigos e diretores da Associação Paulista de Anestesiologia Veterinária APAV, obrigado pelo apoio e pelo esforço dispensado ao bem comum.

À Dra. Denise Aya Otsuki pela ajuda imprescindível, pela paciência e ajuda em todas as fases do projeto. 
Ao Gilberto Nascimento pelo conhecimento e auxílio indispensável na execução do experimento no LIM-08.

À secretaria da pós graduação da anestesiologia, ao Clayton e Claudia pela ajuda e colaboração ao andamento do projeto.

À Fundação de Amparo a Pesquisa do Estado de São Paulo - FAPESP, pelo apoio financeiro imprescindível para a execução desta pesquisa 
SUMÁRIO

Resumo

Abstract

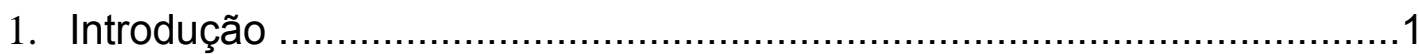

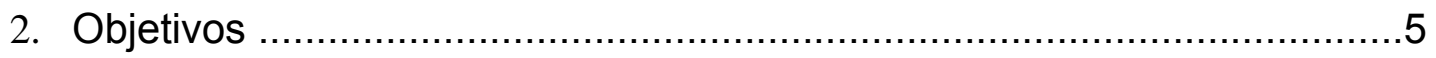

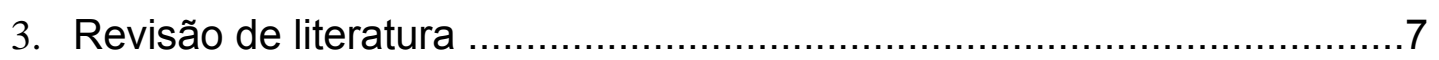

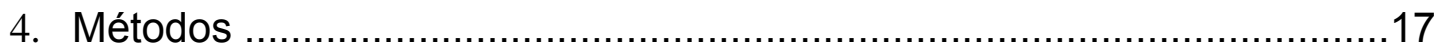

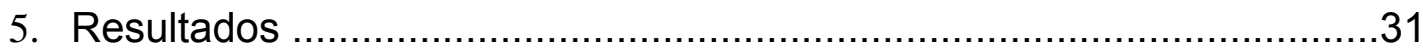

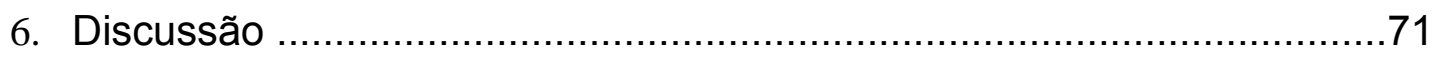

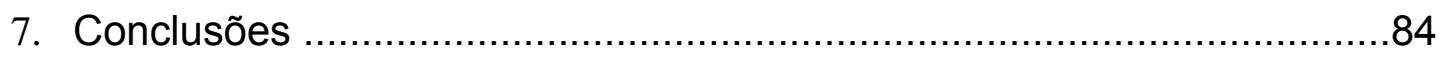

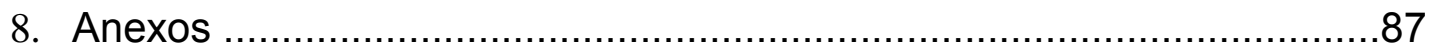

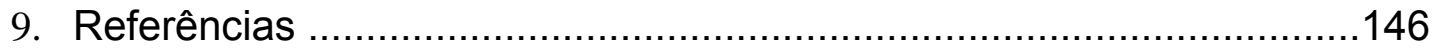




\section{Resumo}

Sant'Ana, A.J. Avaliação da responsividade cardiovascular por meio da variação de pulso com diferentes fluídos em modelo experimental de hemodiluição normovolêmica aguda. São Paulo, 2008. Tese (doutorado) - Faculdade de medicina da Universidade de São Paulo.

Introdução: $A$ variação da pressão de pulso $(\triangle \mathrm{PP})$ tem se mostrado ser uma ferramenta indicadora da responsividade à terapia de fluídos em estudos clínicos e experimentais. Entretanto, esses parâmetros ainda não foram adequadamente estudados em situações de variação aguda da viscosidade sanguínea, como ocorre durante hemodiluição normovolêmica aguda (HNA). O propósito desse estudo é comparar o comportamento do $\triangle P P$ em modelo experimental de HNA em suínos com reposição volêmica com solução salina ou colóide. Métodos: Vinte e um suínos de peso médio de $32 \mathrm{~kg}$ foram anestesiados e randomizados em três grupos de estudo: HES, o sangue removido foi reposto com solução de amido hidroxietílico $6 \%$; SS, solução salina a $0,9 \%$ utilizada na reposição volêmica, e grupo controle, no qual os animais foram somente anestesiados. Parâmetros hemodinâmicos foram mensurados no início do procedimento (basal) (T0), metade processo de HNA (T2), 1 hora e 2 horas depois da HNA (T3 e T4 respectivamente), e imediatamente e 1 hora depois da retransfusão sanguínea (T5, T6). Os dados foram submetidos à análise de variância para medidas repetidas, seguida de teste de Tukey. As distribuições das variáveis mensuradas em mais de uma condição de avaliação, sem distribuição normal, foram comparadas com o teste Friedman e os grupos foram comparados com o teste de Mann-Whitney. Os valores de $p<0,05$ foram considerados estatisticamente significantes. Resultados: Depois da HNA, os parâmetros dinâmicos de responsividade a reposição volêmica, representados pelo $\triangle \mathrm{PP}$, foram diferentes quando os animais foram tratados com solução colóide ou cristalóide (6,1 $\pm 2,5$ e 14,1 \pm 3,4 respectivamente). Os parâmetros estáticos tais como pressão de oclusão de artéria pulmonar (HES $11 \pm 3$; SS $8 \pm 3$ ) e pressão venosa central (HES $8 \pm 2$; SS $7 \pm 4$ ) são contraditórios quando comparados com $\triangle P P$. Conclusão: Durante o processo de HNA a solução colóide mantém os valores de $\triangle \mathrm{PP}$ próximos ao basal quando comparado com o grupo solução salina, provavelmente devido à diferença na meia vida do HES e da solução salina no espaço intravascular.

Descritores: pulso, hemodiluição, solução salina, amido, suínos. 


\begin{abstract}
Sant'Ana, A.J. Assessment of cardiovascular responsiveness through variation in pulse with different fluids in an experimental model of acute normovolemic hemodilution. São Paulo, 2008. Doctoral thesis - School of Medicine of the São Paulo University.

Background: Pulse pressure variation (PPV), have been shown to be useful tools to indicate fluid responsiveness in clinical and experimental studies. However, this parameter has not been adequately explored in situations of acute variation of blood viscosity, as happens in acute normovolemic hemodilution ( $\mathrm{ANH})$. The purpose of this research was to compare the behavior of PPV in a porcine model of ANH replaced with either normal saline or starch. Methods: Twenty one pigs $32 \mathrm{Kg}$ were anesthetized and randomly allocated in three groups: HES, blood removal was replaced with $6 \%$ hydroxyethyl starch; NS, normal saline was utilized as fluid replacement, and CONTROL, animals remained anesthetized without any intervention. Hemodynamic parameters were measured at baseline (T0), middle of ANH (T1), immediately after ANH (T2), $1 \mathrm{~h}$ and $2 \mathrm{~h}$ after ANH (T3 and T4 respectively), and immediately and $1 \mathrm{~h}$ after blood re-infusion (T5,T6). Data were submitted to analysis of variance for repeated measures followed by Tukey test or Friedman test followed by Mann-Whitney test when appropriate. $\mathrm{P}<0.05$ was considered statistically significant. Results: After ANH, dynamic parameters of fluid responsiveness, herein represented by PPV, presented different response when animals were treated with either starch $(6,1 \pm 2,2)$ or saline $(14,1 \pm 3,4)$. Besides, static parameters: pulmonary artery wedge pressure (HES $11 \pm 3 ; 8 \pm 3$ ) and central venous pressure (HES $8 \pm 2$; NS $7 \pm 4$ ) presented contradictory values when compared to PPV. Conclusion: During ANH process colloids restored PPV values to baseline when compared o to normal saline, probably due to a difference in half-lives of HES and normal saline in the intravascular space.
\end{abstract}

Descriptors: pulse, haemodilution, saline solution, starch, pigs 
1. INTRODUÇÃO 


\section{INTRODUÇÃO}

O grande desafio no tratamento de pacientes grave é a otimização da perfusão e oxigenação teciduais, sendo a adequação da volemia uma das principais metas. ${ }^{1}$

O paradigma durante a reposição volêmica ou mesmo durante a hemodiluição normovolêmica aguda é a avaliação correta da volemia para compensar a perda de sangue. Até o momento, nesse contexto, não foi definido um parâmetro ideal para mensurar-se a adequação volêmica, sendo comumente avaliadas a pressão de enchimento cardíaco, como a pressão venosa central (PVC) e a pressão de oclusão de artéria pulmonar (POAP) com suas limitações já conhecidas ${ }^{2}$. As alterações da complacência venosa e ventricular assim como disfunção miocárdica podem levar a modificação desses parâmetros. A ecocardiografia trans-esofágica ou trans-torácica também pode ser utilizada com a finalidade de estimar-se a volemia, entretanto dentre suas limitações, citam-se a necessidade de uma boa janela acústica, presença de um especialista e seu alto custo.

Novas modalidades de monitorização hemodinâmica englobadas com as normas de variação da pressão sistólica (VPS) ${ }^{1}$, variação da pressão de pulso $(\triangle \mathrm{PP})$ e variação do volume sistólico (VS) com a ventilação têm sido estudadas e definidas como indicadores dinâmicos de resposta ao tratamento volêmico, podendo ser úteis na otimização da perfusão e oxigenação teciduais ${ }^{3-5}$. 
Entretanto, existe uma lacuna importante que não foi devidamente explorada pela literatura: são os pacientes submetidos a hemodiluição normovolêmica aguda, nos quais os estudos clínicos de monitorização não contemplam especificamente esse aspecto, e poucos ensaios compararam taxas diferentes de hematócrito utilizando-se os parâmetros dinâmicos de responsividade aos fluídos. A HNA tem sido utilizada em vários cenários com o objetivo de se reduzir a transfusão de sangue homólogo e ensaios clínicos confirmam o benefício deste fato ${ }^{6,7}$.

Dados os riscos e a ausência de evidência considerando os benefícios da transfusão alogênica, atualmente as indicações de transfusão não devem ser ditadas por um nível específico de hemoglobina e sim pela capacidade da microcirculação de extração de oxigênio e perfusão tecidual em cada situação. Em pacientes graves, a estratégia liberal de transfusão demonstrou-se ser pior $^{1}$ em relação a sobrevida quando comparada a restritiva, na qual tolera-se menores taxas de hemoglobina. Em publicação de grande impacto, Hébert e colaboradores $(1999)^{8}$, revisaram os principais estudos randomizados, controlados e prospectivos que compararam a estratégia transfusional liberal com a restritiva em pacientes críticos, incluindo os portadores de cardiopatias. O grupo da estratégia liberal apresentou pior evolução clínica quando comparado à estratégia restritiva. Esses resultados sugerem que mesmo os pacientes com doenças cardíacas podem tolerar a hemodiluição normovolêmica aguda ou a anemia aguda desde que a normovolemia seja mantida. 
Como o sucesso da HNA depende quase que exclusivamente da monitoração adequada da volemia, este estudo visa comparar em um modelo já padronizado em nosso laboratório ${ }^{9-12}$, os índices dinâmicos de responsividade aos fluídos ( $\triangle P P$ e VPS) com índices tradicionais de fluxo obtidos pela ecocardiografia e débito cardíaco contínuo. Também serão analisados outros parâmetros como quantidade de água extra vascular pulmonar (EVLW) e volume de sangue intratorácico (ITBV). Como a HNA elícita importante resposta hemodinâmica, altera a elasticidade dos vasos e viscosidade sanguínea e interfere com a volemia central, pretende-se comparar neste modelo a acurácia dos métodos acima e fornecer para a literatura valores derivados da variação da pressão sistólica e variação da pressão de pulso, em ventilação mecânica, nesta situação hemodinâmica. 
2. OBJETIVO $+$ 


\section{OBJETIVO}

O objetivo deste estudo é avaliar os indicadores dinâmicos de responsividade à reposição volêmica durante HNA, com reposição do sangue retirado com fluídos de composições diferentes, cotejando-os com parâmetros hemodinâmicos estáticos.

Também serão avaliados:

- Os parâmetros referentes ao débito cardíaco transpulmonar, água extravascular pulmonar e sangue intratorácico (Monitor Philips Intellivue MP40 - Alemanha).

- Valores de fração de ejeção e volume de câmaras cardíacas analisados pelo ecocardiograma (M2410B-Sistema de Ultrassonografia IMAGE POINT HX - Hewlett Packard). 
3. REVISÃO DE LITERATURA 


\section{REVISÃO DE LITERATURA}

A literatura tem mostrado de maneira consistente que a sobrevida de pacientes clínicos e cirúrgicos de maior gravidade tem relação direta com a otimização da perfusão e oxigenação teciduais ${ }^{13}$. Estudos recentes demonstram que a ressuscitação volêmica precoce e eficaz é fator fundamental na redução de mortalidade e na melhora do prognóstico de pacientes que se encontram em estado perfusional comprometido, ou seja, com desequilíbrio entre oferta e demanda de oxigênio ${ }^{14}$. O sofrimento celular ocorre quando o sistema circulatório não é capaz de manter a perfusão tecidual adequada. As alterações circulatórias, depleção do volume intravascular, vasodilatação periférica, depressão miocárdica e aumento do metabolismo, resultam em desequilíbrio entre oferta sistêmica e demanda de oxigênio, podendo resultar em hipóxia tecidual. Na medida em que isso ocorre, sinais de disfunção orgânica tornam-se evidentes, e se a intervenção terapêutica não for precoce, pode ocorrer dano celular irreversível ${ }^{8}$. Nesse contexto, a adequação da volemia é meta fundamental no tratamento da disfunção do transporte de oxigênio. A expansão volêmica é utilizada com o objetivo de melhorar os parâmetros hemodinâmicos, aumentando a oferta de oxigênio e perfusão tecidual, minimizando a disfunção orgânica. Devido a relação direta entre o volume diastólico ventricular e o volume sistólico, a resposta hemodinâmica esperada à expansão volêmica é o aumento do volume diastólico final do ventrículo direito e esquerdo, com correspondente incremento do volume sistólico e débito cardíaco. A elevação do volume 
sistólico como resultado do aumento do volume diastólico é diretamente dependente da função ventricular. Porém, apenas 40 a $72 \%$ dos pacientes graves respondem à expansão volêmica com aumento significativo do volume sistólico e débito cardíaco ${ }^{2}$. Esse achado enfatiza a necessidade de fatores preditores da resposta volêmica para selecionar pacientes que vão se beneficiar ou não dessa intervenção, para se evitar expansão volêmica ineficaz ou deletéria, que resulte em piora das trocas gasosas, hemodiluição e edema conseqüente ao excesso de fluídos ${ }^{1}$.

Indicadores da pré-carga ventricular são parâmetros clássicos de avaliação da resposta volêmica. Dentre eles, as pressões de enchimento como a pressão venosa central e a pressão de oclusão da artéria pulmonar são os índices mais utilizados para guiar a terapia de reposição volêmica, e já denominados estáticos ${ }^{15}$. Outros indicadores de pré-carga ventricular como o volume diastólico final do ventrículo direito e a área final diastólica do ventrículo esquerdo também têm sido testados como preditores da resposta hemodinâmica à expansão volêmica. Entretanto, as limitações desses índices obtidos pela ecocardiografia na avaliação da volemia vêm estimulando a busca de outros preditores nesse contexto ${ }^{16}$.

Os estudos clínicos têm enfatizado a discrepância dos indicadores de pré-carga ventricular estáticos com os preditores de resposta a provas de volume. Há razões fisiológicas para explicar esses achados. Dentre elas, cita-se o fato de que a pressão venosa central, a pressão de oclusão da artéria pulmonar, o volume diastólico final do ventrículo direito e a área final diastólica do ventrículo esquerdo não representam com acurácia a pré-carga 
ventricular. Além disso, a pressão venosa central e a pressão de oclusão da artéria pulmonar não estimam adequadamente as pressões intramurais em pacientes mecanicamente ventilados, ou com PEEP ou que apresentam PEEP intrínseco. Também a pressão venosa central (PVC) e a pressão de oclusão da artéria pulmonar (POAP) são altamente dependentes da complacência ventricular, os quais estão frequentemente reduzidas nas situações de sepse, isquemia, hipertrofia ventricular ou uso de drogas vasoativas. Por serem as pressões intramurais e não as pressões intracavitárias (PVC ou PAOP) as relacionadas ao volume diastólico final, não é surpresa que esses parâmetros não sejam fidedignos na predição da resposta volêmica ${ }^{17}$.

A avaliação do volume diastólico final do ventrículo direito, pela ecocardiografia, é influenciada pela regurgitação tricúspide, frequentemente encontrada em pacientes com hipertensão pulmonar, e aqueles sob ventilação mecânica com PEEP e lesão pulmonar aguda. Nesse mesmo contexto, a medida da área final diastólica do ventrículo esquerdo nem sempre reflete o volume desta cavidade e por consequência a pré-carga do ventrículo esquerdo. O mesmo se pode dizer do volume final do ventrículo direito pelos cateteres volumétricos de artéria pulmonar.

Na avaliação da volemia, dois aspectos devem ser considerados:

a) o aumento do volume diastólico como resultado da reposição volêmica dependente da distribuição dos fluidos nos diversos compartimentos. 
b) o aumento do volume sistólico resultante do aumento do volume diastólico final dependente da função ventricular desde que a redução na contratilidade miocárdica altere a relação entre volume diastólico e pressão correspondente. Assim, um paciente pode ser não responsivo à expansão volêmica por apresentar alta complacência venosa, baixa complacência ventricular e ou disfunção miocárdica.

Assumindo que as alterações respiratórias na pressão pleural induzem maiores alterações na PVC quando o ventrículo direito encontra-se altamente complacente, Magder (2004) investigou se a redução inspiratória na PVC poderia ser utilizada para predizer resposta a prova volêmica. Dois estudos demonstraram que uma resposta positiva à reposição volêmica era provável em pacientes com redução inspiratória na $\mathrm{PVC} \geq 1 \mathrm{mmHg}$, e improvável se a redução fosse menor que $1 \mathrm{mmHg}$. Mas, a maioria dos pacientes graves ou sob anestesia geral encontram-se em ventilação mecânica, o que os torna incapazes de produzir uma redução suficiente na pressão pleural para reduzir a $\mathrm{PVC}^{18}$.

De acordo com Perel e colaboradores $(1987)^{19}$ com a variação da pressão sistólica (VPS), durante a ventilação mecânica, pode-se também avaliar a resposta à prova volêmica. Tal índice pode ser dividido em dois componentes, Delta up e Delta down, a partir do traçado da pressão arterial sistólica após curto intervalo de apnéia como valor de referência. Entretanto, este parâmetro é muito sensível durante mudanças de volume intravascular como comprovado por Nascimento e colaboradores $(2006)^{20}$, em cães, sem ser necessariamente um bom preditor do volume sanguíneo circulante, pois 
a VPS pode ser influenciada por muitos outros fatores como, por exemplo, diminuição do volume sanguíneo produzido por diminuição da pressão venosa central ou vice versa ${ }^{21}$.

Pela sua aparente simplicidade, a análise do traçado da pressão arterial, a variação da pressão sistólica, ou da pressão de pulso têm tido grande destaque na literatura médica. Carlos e colaboradores (2005) ${ }^{4}$ mostraram sua superioridade em sensibilidade para avaliar a volemia quando comparadas a índices estáticos como a pressão venosa central, pressão de oclusão da artéria pulmonar, volume diastólico final e débito cardíaco após prova de volume em pacientes anestesiados para cirurgia cardíaca.

Outro parâmetro é a variação da pressão de pulso $(\Delta \mathrm{PP})$. Alguns estudos têm mostrado que o $\triangle P P$ é de importante utilidade clínica, sendo em pacientes graves mantidos sob ventilação mecânica, indicador dinâmico bastante sensível de resposta ao tratamento volêmico. ${ }^{22,} 23 \mathrm{Em}$ estudo envolvendo 40 pacientes submetidos à ventilação mecânica com falência circulatória aguda por sepse Michard e colaboradores $(2000)^{3}$ concluíram que o $\triangle \mathrm{PP}$ foi, dentre os métodos de monitorização hemodinâmica, o mais sensível e específico, à terapia de expansão de volume.

A pressão de pulso (PP) é calculada pela pulsação base e diferença entre pressão arterial sistólica e diastólica. O máximo PP (PP max.) e o mínimo PP (PP mín.) são mensurados sobre um único ciclo respiratório. As variações da pressão de pulso $(\Delta \mathrm{PP})$ são calculados sobre o PP max e o PP min e expressa em porcentagem: 


$$
\Delta P P(\%)=100 x \frac{P P \max -P P \min }{(\underline{P P \max +P P \min })}
$$

2

As variações da pressão arterial durante o ciclo respiratório sofrem influência do estado volêmico. Na hipovolemia estas variações são acentuadas em virtude da veia cava ser mais compressível ao aumento da pressão pleural, fenômeno este atenuado com administração de líquidos ${ }^{24,}$ 25. Entretanto, conforme foi ressaltado, os métodos não foram cotejados entre si durante a HNA.

Cottis e colaboradores $(2003)^{26}$ compararam três métodos de monitorização hemodinâmica em pacientes críticos (Tabela 1), catéter de artéria pulmonar, doppler esofágico e análise de contorno de pulso (Pulseinduced contour cardiac output [PiCCO]). O PiCCO é um método no qual se torna possível estimar o volume de sangue intratorácico (ITBV), água extra vascular pulmonar (EVLW) e índice de função cardíaca (CFI). Esses parâmetros são de interesse por serem considerados mais específicos em relação a pré-carga cardíaca, preditores de edema pulmonar, contratilidade cardíaca e indicador global do desempenho cardíaco. 
Tabela 1-Comparação de débito cardíaco pela análise de contorno de pulso (PiCCO),Doppler esofágico e catéter de artéria pulmonar na monitorização hemodinâmica $\left(\right.$ Cottis et al., 2003) ${ }^{26}$

\begin{tabular}{|c|c|c|c|}
\hline & $\underline{\mathrm{PiCCO}}$ & Doppler esofágico & $\begin{array}{c}\text { Cateter de artéria } \\
\text { pulmonar }\end{array}$ \\
\hline $\begin{array}{l}\text { Método de } \\
\text { mensuração }\end{array}$ & $\begin{array}{l}\text { Termodiluição e } \\
\text { análise do contorno } \\
\text { de pulso }\end{array}$ & $\begin{array}{l}\text { Ultrassom } \\
\text { ecocardiográfico } \\
\text { diâmetro de aorta e } \\
\text { determinação de fluxo } \\
\text { pelo Doppler }\end{array}$ & Termodiluição \\
\hline Parâmetros & $\begin{array}{l}\text { - } \text { CO } \\
\text { - } \text { GEDV } \\
\text { - } \text { CFI } \\
\text { - ITBV } \\
\text { - } \text { EVLW } \\
\text { - SV } \\
\text { - SVRI } \\
\text { - } \mathrm{dp} / \mathrm{dt} \\
\text { - } \mathrm{HR} \\
\text { - } \mathrm{AP}\end{array}$ & $\begin{array}{ll}\text { - } & \text { Fluxo sanguíneo na } \\
& \text { aorta descendente } \\
\text { - } & \text { CO para fluxo } \\
& \text { sanguíneo aórtico } \\
\text { - SV } \\
\text { - HR } \\
\text { - SVR } \\
\text { - Estimativa do tempo } \\
\text { de ejeção do } \\
\text { ventrículo esquerdo }\end{array}$ & $\begin{array}{ll}\text { - } & \text { CO } \\
\text { - } & \text { CPV } \\
\text { - } & \text { PAP } \\
\text { - } & \text { POAP } \\
\text { - } & \text { PWP }\end{array}$ \\
\hline Vantagens & $\begin{array}{l}\text { - Débito cardíaco } \\
\text { contínuo } \\
\text { - Não requer acesso } \\
\text { adicional } \\
\text { - Uso pediátrico } \\
\text { - Mensura a água } \\
\text { pulmonar }\end{array}$ & $\begin{array}{l}\text { - Não requer acesso } \\
\text { vascular } \\
\text { - Monitoração } \\
\text { contínua } \\
\text { - Mínimas } \\
\text { complicações }\end{array}$ & $\begin{array}{l}\text { - Aceita variações } \\
\text { clínicas } \\
\text { - Mais preciso na } \\
\text { mensuração }\end{array}$ \\
\hline
\end{tabular}

\begin{tabular}{|c|c|c|c|}
\hline Desvantagens & $\begin{array}{l}\text { - Calibração por } \\
\text { injeção de bolus } \\
\text { - Impreciso em } \\
\text { certas condições } \\
\text { clínicas } \\
\text { - Risco de infecções } \\
\text { e trombose } \\
\text { - Método caro }\end{array}$ & 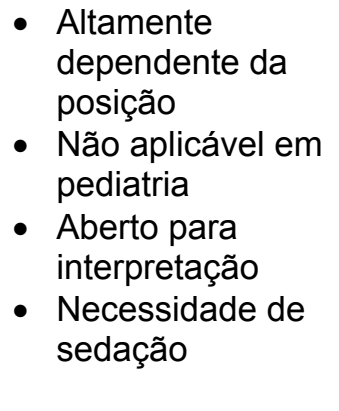 & $\begin{array}{l}\text { - Invasivo } \\
\text { - Influenciado pelo } \\
\text { ciclo respiratório } \\
\text { - Alto risco de } \\
\text { complicações } \\
\text { - Método caro, } \\
\text { demanda tempo e } \\
\text { equipamento } \\
\text { - Não aplicável em } \\
\text { pediatria }\end{array}$ \\
\hline
\end{tabular}

CO-débito cardíaco, GEDV-volume diastólico final global, CFl-índice de função cardíaca, ITBV-volume sangue intratorácico, EVLW-água extra vascular pulmonar, SV-volume sistólico, SVRI- índice resistência vascular sistêmica, dp/dt-mensuração de contratilidade, HR- frequência cardíaca, AP- pressão arterial, CVP-pressão venosa central, PAP-pressão artéria pulmonar, PAOP-pressão oclusão de artéria pulmonar, PWP-pressão em cunha artéria pulmonar. 
Para mensuração do débito cardíaco pelo PiCCO necessita-se de uma linha venosa central como jugular interna ou subclávia e catéter com sensor térmico em vaso arterial de maior calibre, como femoral ou artéria braquial. Salukhe \& Wyncoll $(2002)^{27}$ esclarecem que o PiCCO é um sistema que trabalha com o princípio de conhecimento do volume e temperatura de solução salina injetada no sistema venoso central. A injeção rapidamente se dispersa com o volume sanguíneo cardíaco e pulmonar. Quando o volume sanguíneo passa pelo sensor térmico inserido no sistema arterial a diferença de temperatura é detectada gerando-se uma curva de dissipação. A equação de Stwart Hamilton é aplicada para o cálculo do débito cardíaco. A termodiluição transpulmonar também calcula volume de pré-carga cardíaca, mas o grande benefício da técnica de PiCCO em pacientes críticos é a mensuração do ITBV e o EVLW. O PiCCO possibilita acesso aos valores de ITVB derivados do volume diastólico global final (GEDV) determinados por mensuração pela termodiluição. Lichtwarck-Ascoff e colaboradores (1992) ${ }^{28}$ mostram que ITVB reflete acuradamente o status do volume sanguíneo circulante em pacientes em cuidados intensivos mantidos sob ventilação mecânica, enquanto os padrões mais frequentemente usados, como pressão venosa central e pressão de oclusão de artéria pulmonar, não refletem a précarga cardíaca. Estimativa inadequada da pré-carga cardíaca que resulte em uso inadequado de fluídos e em má perfusão tecidual pode levar a falência de múltiplos órgãos. Por outro lado, fluídos em excesso pode resultar em edema pulmonar, piorando a função respiratória contribuindo para o 
aumento de dias em que o paciente permanece sob ventilação mecânica, maior permanência em tratamento intensivo corroborando para maior morbidade e mortalidade ${ }^{23}$. 
4. MÉTODOS

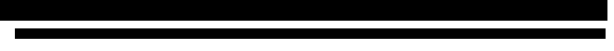




\section{MÉTODOS}

\subsection{LOCAL DE REALIZAÇÃO E COMISSÃO DE ÉTICA}

Este estudo foi realizado no Laboratório de Investigação Médica LIM08 - Laboratório de Anestesiologia Experimental da Faculdade de Medicina da Universidade de São Paulo (FMUSP), com apoio financeiro da Fundação de Amparo à Pesquisa do Estado de São Paulo (FAPSEP), por meio dos processos 06/55221-8 (Auxilio pesquisa), 06/55222-4 (bolsa DD).

O protocolo experimental foi submetido e aprovado pela Comissão de ética para análise de projeto de pesquisa (CAPPesq) do Hospital das Clínicas e Faculdade de Medicina da Universidade de São Paulo - $\mathrm{n}^{\circ}$ $686 / 05$

\subsection{ANIMAIS}

Foram empregados para o estudo 30 suínos com peso médio de 31,8 $\pm 1,2 \mathrm{~kg}$, provenientes de granjas de alto padrão sanitário. Os animais foram previamente submetidos a exame clínico e laboratorial completo sendo descartados aqueles portadores de qualquer anomalia que pudesse interferir no andamento do estudo.

Dos 30 animais, três foram utilizados para estudo piloto e 27 para a fase experimental, dos quais um veio a óbito antes do término do experimento e cinco foram descartados antes da randomização por 
apresentarem alterações hemodinâmicas e metabólicas. Portanto, foram estudados 21 animais divididos em 3 grupos experimentais.

\subsection{PROCEDIMENTOS}

\subsubsection{JEJUM}

Os animais foram submetidos a jejum alimentar de 12 horas e hídrico de duas horas.

\subsubsection{PROCEDIMENTO ANESTÉSICO}

Todos os animais do experimento receberam midazolam ${ }^{1}$ na dose de $0,25 \mathrm{mg} \cdot \mathrm{kg}^{-1}$ associado ao cloridrato de cetamina na dose de $5 \mathrm{mg} \cdot \mathrm{kg}^{-1}$ pela via intramuscular, como medicação pré-anestésica. Decorridos 15 minutos a veia marginal da orelha foi cateterizada com cateter ${ }^{2}$ de calibre $20 \mathrm{G}$ realizada a indução anestésica com propofol na dose de $5 \mathrm{mg} \cdot \mathrm{kg}^{-1}$, intubação orotraqueal com sonda de diâmetro apropriado e manutenção por meio de anestesia inalatória com isofluorano a $1,4 \%$ no ar expirado, iniciando-se então a instrumentação do animal. Após a intubação os animais permaneceram em ventilação controlada na modalidade volume controlado, $10 \mathrm{~mL} / \mathrm{kg}$, e fração inspirada de oxigênio de 40\%. Para tanto,

\footnotetext{
${ }^{1}$ Dormire - Cristália

${ }^{2}$ Abbocath Tplus - Produtos Hospitalares Abbott
} 
administrou-se infusão contínua de pancurônio ${ }^{3}$ na dose de $5 \mu \mathrm{g} \cdot \mathrm{kg}^{-1} \cdot \mathrm{min}^{-1}$ IV. A administração contínua do bloqueador neuromuscular foi feita utilizando-se bombas de infusão para seringas ${ }^{4}$.

A pressão parcial de dióxido de carbono no ar expirado $\left(\mathrm{EtCO}_{2}\right)$ foi mensurada continuamente com auxílio de capnógrafo e a saturação periférica da hemoglobina por meio de oxímetro de pulso $^{5}$ colocado na língua do animal. A pressão parcial de dióxido de carbono foi mantida entre 35 a $45 \mathrm{mmHg}$ por meio de adequação da FR. Os sinais de mecânica respiratória foram obtidos diretamente do ventilador da estação de trabalho (Cícero - Dräger Alemanha) e transferidos para o monitor multiparamétrico (Dixtal DX2020 Brasil) para mensuração do $\Delta$ PP e VPS. Antes de cada procedimento os sensores de pressão e fluxo do ventilador foram calibrados conforme as normas do fabricante.

Após a anestesia e instrumentação os animais foram randomizados em um dos três grupos em estudo.

\subsubsection{FLUIDOTERAPIA}

Os animais receberam fluidoterapia de manutenção com solução fisiológica ${ }^{6}$ na taxa de $5 \mathrm{~mL} \cdot \mathrm{kg}^{-1} \cdot$ hora $^{-1}$. Para assegurar a administração correta de fluido utilizou-se uma bomba de infusão ${ }^{7}$.

\footnotetext{
${ }^{3}$ Tracrium - Glaxo Wellcome

${ }^{4}$ Bomba de infusão - Santronic - Modelo 680

${ }^{5}$ Oximetro de pulso - Viridia Modelo 885 - Hewlett Packard

${ }^{6}$ Solução fisiológica - Baxter Hospitalar Ltda, São Paulo

${ }^{7}$ Bomba de infusão Anne - Abbott, North Chicago
} 


\subsubsection{INSTRUMENTAÇÃO}

\section{Cateter de artéria pulmonar}

Após anestesia, os animais foram posicionados em decúbito dorsal horizontal e realizou-se a dissecção da veia jugular para a introdução do cateter de artéria pulmonar ${ }^{8}$ acoplado à monitoração de $\mathrm{SvO}_{2}$ contínuo. Primeiramente o sistema transdutor-registrador foi preenchido com solução salina heparinizada ${ }^{9}$ e zerado à pressão atmosférica sendo conectado ao transdutor de pressão posicionado ao nível da linha axilar média. Quando se verificou alteração do traçado com "achatamento da curva", a pressão de oclusão da artéria pulmonar foi considerada e o cateter então fixado. Para obtenção da pressão venosa central (PVC), pressão de artéria pulmonar (PAP) e pressão de oclusão de artéria pulmonar (POAP) foi utilizado monitor multiparamétrico. Para mensuração do débito cardíaco em cada momento de observação, se fez uso de solução glicosada mantida entre $3^{\circ} \mathrm{C}$ sendo essa indicadora para registro no sensor de termodiluição do monitor Vigilance - Edwards Lifesciences- USA. A solução é injetada pela via de PVC do cateter de artéria pulmonar sendo registrada pelo sensor da extremidade distal do cateter.

\section{Cateter de pressão arterial sistêmica e PICCO}

Um cateter de polietileno ${ }^{10}$ de calibre $5 \mathrm{~F}$ foi introduzido, por dissecção, na artéria femoral e acoplado a outro transdutor de pressão para

\footnotetext{
${ }^{8}$ Swan Ganz Thermodilution and continuos $\mathrm{SvO}_{2}$ Catheter Model 744H-7,5F - Baxter Healthcare Corporation, CA, USA

${ }^{9}$ Liquemine - Produtos Roche Quimicos e Farmaceuticos S.A.

${ }^{10}$ Pulsiocath PV 2015L20
} 
obtenção da pressão arterial sistêmica pelo Monitor Philips Intellivue MP40 (Alemanha).

O mesmo cateter foi utilizado para o método transpulmonar, utilizando-se o catéter de artéria pulmonar (via proximal) para injeção da solução indicadora (solução glicosada $3^{\circ} \mathrm{C}$ ).

\section{Cateter para retirada de sangue:}

A veia e a artéria femoral do membro contra-lateral foram dissecadas para introdução de tubo de polietileno para a retirada do sangue e infusão de fluído durante o processo de hemodiluição.

\subsubsection{PROCEDIMENTO EXPERIMENTAL}

Após a estabilização da anestesia e concluída a instrumentação, foi realizada a infusão de $20 \mathrm{~mL}$. $\mathrm{Kg}^{-1}$ de solução salina $0,9 \%$ em todos os animais, antes da randomização, com o objetivo de otimização da volemia, repondo as perdas volêmicas por jejum e transporte, colocando os animais em um mesmo "patamar" volêmico. Após uma hora da estabilização da anestesia, os animais foram randomizados nos 3 grupos sendo nesse momento coletados os parâmetros basais hemodinâmicos, respiratórios, metabólicos e ecocardiográficos, bem como o hematócrito(T0). Procedeu-se então ao cálculo de volume de sangue a ser retirado e o volume do fluído de reposição, caso fosse esse o grupo a ele atribuído.

Os animais foram distribuídos em 3 grupos: 
Grupo 1 (Controle): Grupo controle, sem hemodiluição, mantido com fluidoterapia de solução salina $0,9 \% 5 \mathrm{~mL} \cdot \mathrm{kg}^{-1} \cdot \mathrm{hora}^{-1}$.

Grupo 2 (HES): submetido à hemodiluição normovolêmica aguda com utilização de solução de amido hidroxietílico ${ }^{11}(130 / 0,4)$ na proporção de 1 $\mathrm{mL}$ para cada $\mathrm{mL}$ de sangue retirado;

Grupo 3 (Solução salina $0,9 \%$ ): submetido à hemodiluição com a utilização de solução fisiológica na proporção de $3 \mathrm{~mL}$ de solução para cada $\mathrm{mL}$ de sangue retirado;

Para o cálculo do volume de sangue a ser retirado (V) foi empregada a fórmula proposta por GROSS $(1983)^{29}$ :

$$
\begin{aligned}
& V=\text { VES } X(\mathrm{Hi}-\mathrm{Hd}) / \mathrm{Hm} \text { onde: } \\
& \text { VES = volume estimado de sangue, sendo considerado } 80
\end{aligned}
$$
$\mathrm{ml} / \mathrm{kg}$ para o suíno adulto; $\mathrm{Hi}=$ hematócrito inicial do paciente; $\mathrm{Hd}=$ hematócrito desejado; $\mathrm{Hm}=$ média entre $\mathrm{Hi}$ e $\mathrm{Hd}$.

O hematócrito final da hemodiluição foi fixado em $15 \%$, sendo feito por etapas:

1) Na primeira etapa da hemodiluição o hematócrito foi reduzido para valores ao redor de $22 \%$ em 30 minutos sendo então anotado os parâmetros (T1).

2) Na segunda etapa, procedeu-se a retirada de sangue em mais 30 minutos, sendo o hematócrito levado a $15 \%$. O volume total de sangue calculado inicialmente foi retirado em 60 minutos (T2).

${ }^{11}$ Voluven - Fresenius 
Após a hemodiluição, os animais foram avaliados durante 120 minutos e os parâmetros mensurados aos 60 e 120 minutos (T3 e T4 respectivamente).

Após esse período $50 \%$ do sangue retirado foi reinfundido em 30 minutos (T5), mantendo-se observação por mais 60 minutos (T6), desta forma simulamos um procedimento cirúrgico onde houve sangramento e posterior reinfusão do sangue estocado. Procedeu-se administração do mesmo fluido empregado na hemodiluição de cada grupo (solução salina ou amido hidroxietílico) para manutenção da pressão arterial quando esta diminuiu mais de $20 \%$ em relação ao valor basal. A quantidade de fluído infundida em cada grupo foi anotada para comparação.

Após o período de observação e coleta dos dados foi realizado o sacrifício dos animais com o aumento da concentração de isoflurano, seguida de $3 \mathrm{~mL}$ de cloreto de potássio.

\subsubsection{AVALIAÇÃO PARAMÉTRICA}

\section{Parâmetros hemodinâmicos}

\section{o Frequência e ritmo cardíacos}

No animal não anestesiado a frequência cardíaca (FC) foi mensurada por meio de ausculta dos batimentos cardíacos em um minuto. Durante o decorrer da anestesia a frequência e ritmo 
cardíaco foram obtidos do monitor cardíaco (Monitor Dixtal DX2020 -Brasil)

\section{o Pressão arterial sistêmica}

A pressão arterial foi obtida por mensuração direta com o cateter da artéria femoral acoplado ao transdutor de pressão do monitor (Monitor Philips Intellivue MP40 - Alemanha), obtendo-se desta forma a pressão arterial média (PAM), sistólica (PAS) e diastólica (PAD), e suas variáveis $\triangle P P$ e VPS.

o Pressão de átrio direito (PAD), pressão da artéria pulmonar (PAP), pressão de oclusão da artéria pulmonar (POAP) Para a obtenção da pressão do átrio direito a via proximal do cateter de Swan-Ganz foi conectada ao transdutor de pressão. A pressão da artéria pulmonar foi obtida ao se conectar a via distal do cateter de Swan-Ganz ao transdutor de pressão. A pressão de oclusão da artéria pulmonar foi obtida insuflando-se o balão localizado na extremidade distal do cateter de Swan-Ganz.

As medidas foram feitas na expiração.

\section{o Débito cardíaco (DC)}

O DC foi obtido por meio de:

- Termodiluição (cateter de artéria pulmonar). Foram realizadas três medidas consecutivas sendo desprezada aquela cujo valor foi discrepante (maior que 10\%).

- Débito cardíaco contínuo (DCC; cateter de artéria pulmonar) 
- Termodiluição transpulmonar (PiCCO)

- DCC pela análise de contorno de pulso (PiCCO).

Sendo utilizado para comparação entre si os métodos de termodiluição (convencional) e termodiluição transpulmonar (PICCO).

o Volume de sangue intratorácico (VSIT) e conteúdo extravascular de água pulmonar (CEVAP)

Foram obtidos diretamente pelo "display" do Monitor Philips Intellivue MP40 - Alemanha, a cada mensuração do débito cardíaco.

o Os parâmetros referentes ao:

Índice Sistólico (IS), índice cardíaco (IC), Índice da Resistência Vascular Sistêmica (IRVS), Índice da Resistência Vascular Pulmonar (IRVP) foram obtidos do monitor Vigilance - Edwards Lifesciences (USA), calculada por fórmula padrão.

o Variação de pressão Sistólica (VPS) e variação de pressão de pulso $(\triangle P P)$

Os valores de VPS e $\triangle P P$ foram obtidos por meio do Monitor Dixtal DX2020 (Brasil) que foi acoplado ao sistema arterial por meio de torneira de 3 vias. 


\section{Parâmetros de oxigenação e eletrólitos}

Foi realizada a coleta de amostra de sangue arterial e venoso misto para análise dos gases sanguíneos e eletrólitos pelo Gasômetro ABLRadiometer (Copenhagen)
- $\mathrm{PaO}_{2}$
- $\mathrm{HCO}^{3-}$
- $\mathrm{PaCO}_{2}$
- $\mathrm{Na}^{+}$
- $\mathrm{SaO}_{2}$
- $\mathrm{K}^{-}$
- $\mathrm{PvO}_{2}$
- lactato
- $\mathrm{SvO}_{2}$
- $\mathrm{pH}$

\section{o Os parâmetros referentes ao:}

Índice de Consumo de Oxigênio $\left(\mathrm{IVO}_{2}\right)$, Índice da Oferta de Oxigênio $\left(\mathrm{IDO}_{2}\right)$, Taxa de Extração de Oxigêno $\left(\mathrm{TEO}_{2}\right)$ e Diferença artério-venosa do oxigênio $\left[\mathrm{C}(\mathrm{A}-\mathrm{V}) \mathrm{O}_{2}\right]$ foram calculados a partir das fórmulas padrões ${ }^{30}$.

\section{Estudo ecodopplercardiográfico}

O estudo ecodopplercardiográfico foi realizado, por profissional habilitado, por meio de sistema de ultrassonografia (M2410B-Sistema de Ultrassonografia IMAGE POINT HX - Hewlett Packard) com transdutor transesofágico de 7,5/5,0 MHz . 


\section{o Análise da fração de ejeção}

A fração de ejeção foi calculada por planimetria do ventrículo esquerdo (Método de Simpson), com o transdutor na posição média do coração, o que permitiu a visualização das quatro câmaras.

\section{Hematócrito e hemoglobina}

Os valores de hematócrito e hemoglobina foram obtidos do sangue coletado na artéria femoral e analisados por microhematócrito, centrifugados em alta rotação por 5 minutos em tubo capilar.

\section{Débito urinário}

O débito urinário foi mensurado por meio de cateterização vesical transuretral nas fêmeas e transvesical por laparotomia nos machos. 


\subsubsection{DELINEAMENTO DO EXPERIMENTO}

O delineamento do experimento está exposto no diagrama abaixo:

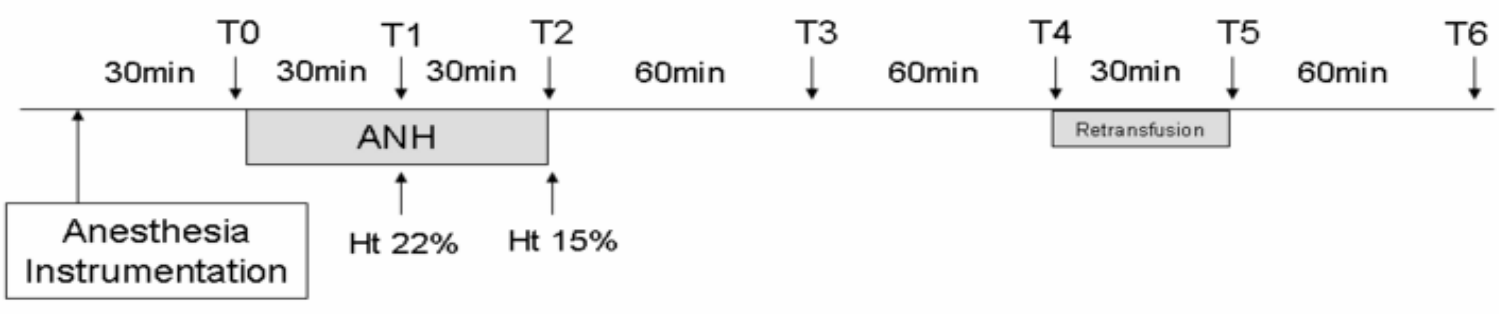

HES 1:1

$\mathrm{NaCl} 0,9 \% 1: 3$

Em cada tempo ( $T$ ) foram mensurados todos os parâmetros hemodinâmicos (dinâmicos e estáticos), de oxigenação e hematócrito. 


\subsection{ANÁLISE ESTATÍSTICA}

As variáveis com distribuição normal foram apresentadas descritivamente em tabelas contendo média, desvio padrão.

As médias das variáveis mensuradas em mais de uma condição de avaliação, com distribuição normal, foram avaliadas com análise de variância para medidas repetidas, onde as seguintes hipóteses básicas foram testadas:

$\mathrm{H}_{01}$ : Os perfis de médias são paralelos, ou seja, o comportamento dos grupos é igual ao longo do tempo;

$\mathrm{H}_{02}$ : Os perfis de médias são coincidentes, ou seja, não existe diferença de médias entre os grupos;

$\mathrm{H}_{03}$ : Não há efeito de tempo, ou seja, os perfis são paralelos ao eixo das abscissas.

Quando a hipótese $\mathrm{H}_{01}$ foi rejeitada, foram realizados contrastes entre grupos e tempos para discriminar as diferenças.

As distribuições das variáveis mensuradas em mais de uma condição de avaliação, sem distribuição normal, foram comparados com o teste de Friedman e os grupos foram comparados com o teste de Mann-Whitney.

Os valores de $p<0,05$ foram considerados estatisticamente significantes. $^{31}$ 
5. RESULTADOS

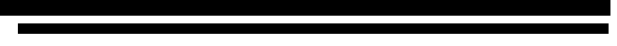




\section{RESULTADOS \\ Balanço hídrico:}

Os valores de peso, superfície corporal e balanço hídrico estão descritos na tabela 2. Não houve diferença entre os grupos em relação ao peso e à superfície corporal. Quanto à produção de urina, houve diferença significativa entre o grupo hemodiluído com solução salina a $0,9 \%$ e os demais grupos.

O volume de sangue retirado, calculado a partir do peso e hematócrito inicial não diferiu entre os grupos. O volume de fluido administrado durante e após a hemodiluição apresentou diferença significativa entre os grupos $(p<0,05)($ Tabela 2$)$.

Tabela 2 - Peso, superfície corporal, balanço hídrico dos animais do grupo Controle e submetidos à hemodiluição com amido hidroxietílico (HES) ou Solução salina $0,9 \%$ (SS) (média \pm desvio padrão)

\begin{tabular}{|c|c|c|c|c|c|c|}
\hline Grupo & Peso (kg) & $\begin{array}{c}\text { Superfície } \\
\text { Corporal } \\
\left(\mathrm{m}^{2}\right)\end{array}$ & $\begin{array}{c}\text { Débito } \\
\text { urinário }(\mathrm{ml})\end{array}$ & $\begin{array}{c}\text { Sangue } \\
\text { retirado } \\
(\mathrm{ml})\end{array}$ & $\begin{array}{c}\text { Volume } \\
\text { infundido } \\
\text { (ml) }\end{array}$ & $\begin{array}{c}\text { Volume } \\
\text { pós HD } \\
(\mathrm{ml})\end{array}$ \\
\hline
\end{tabular}

Controle $31,4 \pm 2,9 \quad 0,89 \pm 0,06 \quad 342 \pm 66$

$\begin{array}{lcccccc}\text { HES } & 32 \pm 1,6 & 0,90 \pm 0,03 & 641 \pm 338 & 1462 \pm 146 & 1462 \pm 146 & 200 \\ & & & & & & \\ \text { SS } & 32,1 \pm 3,2 & 0,91 \pm 0,06 & 2643 \pm 1097 & 1394 \pm 315 & 4183 \pm 945 & 900 \pm 668\end{array}$


Os dados hemodinâmicos obtidos diretamente, bem como aqueles calculados por meio das fórmulas padrões, os de oxigenação e ecocardiografia transesofágica estão descritos nas tabelas abaixo.

Os dados individuais de todos os parâmetros avaliados estão dispostos em tabelas no item ANEXOS. 
Tabela 3 - Parâmetros hemodinâmicos dos animais do grupo Controle e submetidos à hemodiluição com amido hidroxietílico (HES) ou solução salina $0,9 \%$ (SS) (média \pm desvio padrão)

\begin{tabular}{|c|c|c|c|c|c|c|c|c|}
\hline \multicolumn{9}{|c|}{ Tempo } \\
\hline Parâmetro & Grupo & TO & T1 & T2 & T3 & T4 & T5 & T6 \\
\hline $\begin{array}{c}\mathrm{FC} \\
\mathrm{Bpm}\end{array}$ & $\begin{array}{c}\text { Controle } \\
\text { HES } \\
\text { SS }\end{array}$ & $\begin{array}{l}104 \pm 16 \\
115 \pm 16 \\
114 \pm 10\end{array}$ & $\begin{array}{c}103 \pm 13 \\
129 \pm 18^{\star} \\
137 \pm 15^{\star a}\end{array}$ & $\begin{array}{c}103 \pm 15 \\
143 \pm 24^{*} \\
145 \pm 20^{* a}\end{array}$ & $\begin{array}{c}102 \pm 17 \\
140 \pm 24^{*} \\
145 \pm 18^{* a}\end{array}$ & $\begin{array}{c}111 \pm 16 \\
137 \pm 23^{\star} \\
142 \pm 17^{\star a}\end{array}$ & $\begin{array}{c}117 \pm 20 \\
133 \pm 20^{*} \\
135 \pm 12^{\star a}\end{array}$ & $\begin{array}{c}124 \pm 23 \\
132 \pm 16^{*} \\
134 \pm 11^{*}\end{array}$ \\
\hline $\begin{array}{l}\text { PAM } \\
\mathrm{mmHg}\end{array}$ & $\begin{array}{c}\text { Controle } \\
\text { HES } \\
\text { SS }\end{array}$ & $\begin{array}{l}89 \pm 20 \\
84 \pm 16 \\
81 \pm 11\end{array}$ & $\begin{array}{c}90 \pm 19 \\
89 \pm 8 \\
81 \pm 12\end{array}$ & $\begin{array}{l}86 \pm 17 \\
89 \pm 10 \\
78 \pm 15\end{array}$ & $\begin{array}{c}88 \pm 13 \\
84 \pm 16 \\
76 \pm 9\end{array}$ & $\begin{array}{c}87 \pm 11 \\
87 \pm 17 \\
75 \pm 9\end{array}$ & $\begin{array}{c}88 \pm 10 \\
97 \pm 10^{\star} \\
87 \pm 8^{*}\end{array}$ & $\begin{array}{c}89 \pm 12 \\
91 \pm 16 \\
80 \pm 8\end{array}$ \\
\hline $\begin{array}{c}\text { PVC } \\
\mathrm{mmHg}\end{array}$ & $\begin{array}{c}\text { Controle } \\
\text { HES } \\
\text { SS }\end{array}$ & $\begin{array}{l}7 \pm 3 \\
6 \pm 2 \\
6 \pm 2\end{array}$ & $\begin{array}{l}7 \pm 3 \\
7 \pm 2 \\
6 \pm 2\end{array}$ & $\begin{array}{l}6 \pm 2 \\
8 \pm 2^{*} \\
7 \pm 4\end{array}$ & $\begin{array}{l}6 \pm 2 \\
5 \pm 2 \\
5 \pm 2\end{array}$ & $\begin{array}{l}5 \pm 2 \\
5 \pm 2^{*} \\
5 \pm 3^{*}\end{array}$ & $\begin{array}{c}6 \pm 3 \\
8 \pm 1^{\star} \\
8 \pm 3^{* a}\end{array}$ & $\begin{array}{l}6 \pm 2 \\
6 \pm 1 \\
6 \pm 3\end{array}$ \\
\hline $\begin{array}{l}\text { PAPM } \\
\mathrm{mmHg}\end{array}$ & $\begin{array}{c}\text { Controle } \\
\text { HES } \\
\text { SS }\end{array}$ & $\begin{array}{l}21 \pm 5 \\
18 \pm 2 \\
18 \pm 3\end{array}$ & $\begin{array}{l}21 \pm 5 \\
19 \pm 3 \\
20 \pm 4\end{array}$ & $\begin{array}{l}17 \pm 8 \\
20 \pm 5 \\
20 \pm 7\end{array}$ & $\begin{array}{l}20 \pm 5 \\
19 \pm 8 \\
19 \pm 5\end{array}$ & $\begin{array}{l}19 \pm 4 \\
18 \pm 6 \\
19 \pm 5\end{array}$ & $\begin{array}{l}22 \pm 5 \\
24 \pm 7^{\star} \\
24 \pm 5^{\star}\end{array}$ & $\begin{array}{l}25 \pm 7^{*} \\
20 \pm 5^{*} \\
20 \pm 4^{*}\end{array}$ \\
\hline $\begin{array}{l}\text { POAP } \\
\mathrm{mmHg}\end{array}$ & $\begin{array}{c}\text { Controle } \\
\text { HES } \\
\text { SS }\end{array}$ & $\begin{array}{c}10 \pm 3 \\
9 \pm 2 \\
9 \pm 2\end{array}$ & $\begin{array}{l}11 \pm 4 \\
10 \pm 3 \\
10 \pm 3\end{array}$ & $\begin{array}{c}11 \pm 3 \\
11 \pm 3^{*} \\
9 \pm 3^{a b}\end{array}$ & $\begin{array}{l}11 \pm 4 \\
7 \pm 2^{\star a} \\
7 \pm 2^{a}\end{array}$ & $\begin{array}{l}11 \pm 5 \\
7 \pm 2^{\star a} \\
7 \pm 3^{a}\end{array}$ & $\begin{array}{l}10 \pm 4 \\
10 \pm 3 \\
10 \pm 3\end{array}$ & $\begin{array}{l}12 \pm 4 \\
8 \pm 2 \\
8 \pm 4\end{array}$ \\
\hline$\frac{\mathrm{IC}}{\mathrm{L} \cdot \mathrm{min}^{-1} \cdot \mathrm{m}^{-2}}$ & $\begin{array}{c}\text { Controle } \\
\text { HES } \\
\text { SS }\end{array}$ & $\begin{array}{l}5,1 \pm 0,3 \\
5,5 \pm 0,6 \\
5,3 \pm 0,6\end{array}$ & $\begin{array}{c}4,6 \pm 0,3 \\
7,2 \pm 0,5^{\star a} \\
7 \pm 1,4^{\star a}\end{array}$ & $\begin{array}{c}4,7 \pm 0,5 \\
8,6 \pm 0,8^{\star a} \\
7,3 \pm 1^{\star a b}\end{array}$ & $\begin{array}{l}4,8 \pm 0,3 \\
7 \pm 0,9^{* a} \\
5,9 \pm 1^{* a b}\end{array}$ & $\begin{array}{c}4,7 \pm 0,7 \\
6,7 \pm 0,7^{\star a} \\
5,6 \pm 0,9^{\mathrm{ab}}\end{array}$ & $\begin{array}{c}4,5 \pm 0,7 \\
6,9 \pm 0,8^{\star a} \\
6,7 \pm 1,1^{* a}\end{array}$ & $\begin{array}{c}4,6 \pm 0,6 \\
6,4 \pm 0,9^{\star a} \\
6,5 \pm 1,1^{* a}\end{array}$ \\
\hline $\begin{array}{c}\text { IRVS } \\
\text { dinas.s.cm } \\
{ }_{5} \mathrm{~m}^{-2}\end{array}$ & $\begin{array}{c}\text { Controle } \\
\text { HES } \\
\text { SS }\end{array}$ & $\begin{array}{l}1333 \pm 255 \\
1120 \pm 141 \\
1136 \pm 167\end{array}$ & $\begin{array}{l}1469 \pm 405 \\
927 \pm 107^{\star a} \\
856 \pm 163^{\star a}\end{array}$ & $\begin{array}{l}1423 \pm 274 \\
764 \pm 139^{\star a} \\
783 \pm 146^{\star^{a}}\end{array}$ & $\begin{array}{l}1455 \pm 238 \\
916 \pm 222^{\star a} \\
981 \pm 193^{\star a}\end{array}$ & $\begin{array}{c}1400 \pm 205 \\
1029 \pm 311^{a} \\
1012 \pm 184^{a}\end{array}$ & $\begin{array}{c}1422 \pm 172 \\
1056 \pm 245^{\mathrm{a}} \\
961 \pm 137^{\star^{\mathrm{a}}}\end{array}$ & $\begin{array}{c}1439 \pm 208 \\
1087 \pm 292^{a} \\
933 \pm 134^{*^{a}}\end{array}$ \\
\hline $\begin{array}{c}\text { IRVP } \\
\text { dinas.S.cm } \\
{ }^{5} \cdot \mathrm{m}^{-2}\end{array}$ & $\begin{array}{c}\text { Controle } \\
\text { HES } \\
\text { SS }\end{array}$ & $\begin{array}{l}163 \pm 47 \\
131 \pm 31 \\
149 \pm 44\end{array}$ & $\begin{array}{l}201 \pm 70 \\
101 \pm 44^{\mathrm{a}} \\
115 \pm 40^{\mathrm{a}}\end{array}$ & $\begin{array}{c}185 \pm 58 \\
90 \pm 44^{\star a} \\
136 \pm 75^{\text {ab }}\end{array}$ & $\begin{array}{c}184 \pm 37 \\
140 \pm 85^{\mathrm{a}} \\
162 \pm 49\end{array}$ & $\begin{array}{c}177 \pm 32 \\
139 \pm 76 \\
180 \pm 82^{b}\end{array}$ & $\begin{array}{c}229 \pm 56 \\
165 \pm 109^{a} \\
171 \pm 51^{a}\end{array}$ & $\begin{array}{l}253 \pm 111 \\
139 \pm 65^{a} \\
156 \pm 36^{a}\end{array}$ \\
\hline $\begin{array}{l}\text { IVS } \\
\mathrm{ml} \cdot \mathrm{m}^{-2}\end{array}$ & $\begin{array}{c}\text { Controle } \\
\text { HES } \\
\text { SS }\end{array}$ & $\begin{array}{l}50 \pm 5 \\
49 \pm 5 \\
47 \pm 5\end{array}$ & $\begin{array}{c}45 \pm 6 \\
56 \pm 8^{\star a} \\
52 \pm 6^{\star a}\end{array}$ & $\begin{array}{c}44 \pm 5 \\
62 \pm 12^{\star a} \\
51 \pm 10^{a b}\end{array}$ & $\begin{array}{c}45 \pm 4 \\
51 \pm 10^{\mathrm{a}} \\
41 \pm 7^{\star \mathrm{b}}\end{array}$ & $\begin{array}{c}42 \pm 3 \\
50 \pm 9^{a} \\
40 \pm 8^{\star b}\end{array}$ & $\begin{array}{c}40 \pm 4 \\
53 \pm 10^{\star a} \\
50 \pm 7^{a}\end{array}$ & $\begin{array}{c}38 \pm 2 \\
49 \pm 10^{a} \\
49 \pm 8^{a}\end{array}$ \\
\hline
\end{tabular}

$\overline{F C \text { : frequência cardíaca; PAM: pressão arterial média; PVC: pressão venosa central; PAPM: pressão }}$ de artéria pulmonar média; POAP: pressão de artéria pulmonar ocluída; IC: índice cardíaco; IRVS: índice de resistência vascular sistêmica;IRVP: índice de resistência vascular pulmonar; IVS: índice de volume sistólico; T0: valor basal, início hemodiluição; T1: 30 minutos início hemodiluição; T2: fim hemodiluição; T3: 60 minutos após a hemodiluição; T4: 120 minutos pós hemodiluição, início retransfusão; T5: fim retransfusão; T6: 60 minutos pós retransfusão * $p<0,05$ diferente de T0; ${ }^{a} p<$ 0,05 diferente do grupo Controle; ${ }^{b} p<0,05$ diferente do grupo HES. 
Tabela 4 - Gases sanguíneos e eletrólitos dos animais do grupo Controle e submetidos à hemodiluição com amido hidroxietílico (HES) ou Solução salina 0,9\% (SS) (média \pm desvio padrão)

\begin{tabular}{|c|c|c|c|c|c|c|c|c|}
\hline \multicolumn{9}{|c|}{ Tempo } \\
\hline Parâmetro & Grupo & TO & T1 & T2 & T3 & T4 & T.5 & T6 \\
\hline $\begin{array}{c}\mathrm{PaO}_{2} \\
\mathrm{mmHg}\end{array}$ & $\begin{array}{c}\text { Controle } \\
\text { HES } \\
\text { SS }\end{array}$ & $\begin{array}{c}148 \pm 17,01 \\
153,31 \pm 41,48 \\
144,37 \pm 44,94\end{array}$ & $\begin{array}{c}145,54 \pm 7,27 \\
149,84 \pm 27,64 \\
143,16 \pm 51,73\end{array}$ & $\begin{array}{l}140,34 \pm 17,31 \\
163,86 \pm 29,29 \\
143,49 \pm 48,19\end{array}$ & $\begin{array}{l}143,44 \pm 20,02 \\
161,03 \pm 27,13 \\
146,16 \pm 45,36\end{array}$ & $\begin{array}{l}142,73 \pm 21,66 \\
161,09 \pm 32,31 \\
149,31 \pm 42,97\end{array}$ & $\begin{array}{l}129,69 \pm 28,83 \\
160,96 \pm 31,83 \\
143,23 \pm 45,06\end{array}$ & $\begin{array}{l}131,30 \pm 28,64^{*} \\
147,53 \pm 27,93^{*} \\
133,07 \pm 41,50^{*}\end{array}$ \\
\hline $\begin{array}{l}\mathrm{PaCO}_{2} \\
\mathrm{mmHg}\end{array}$ & $\begin{array}{c}\text { Controle } \\
\text { HES } \\
\text { SS }\end{array}$ & $\begin{array}{c}44 \pm 3,4 \\
42,7 \pm 3,1 \\
45 \pm 3,5\end{array}$ & $\begin{array}{l}42,8 \pm 3,4 \\
44,4 \pm 2,4 \\
47,7 \pm 9,8\end{array}$ & $\begin{array}{l}44,6 \pm 3,9 \\
45,4 \pm 4,1 \\
47,6 \pm 3,1\end{array}$ & $\begin{array}{c}46 \pm 4,3 \\
44,9 \pm 4,1 \\
47,5 \pm 5,6\end{array}$ & $\begin{array}{c}43,8 \pm 3,1 \\
45,4 \pm 4,8 \\
48,4 \pm 4\end{array}$ & $\begin{array}{c}45,4 \pm 5,5 \\
45,8 \pm 4,4 \\
49 \pm 2,8\end{array}$ & $\begin{array}{l}41,9 \pm 4,6 \\
45,3 \pm 4,6 \\
48,4 \pm 5,2\end{array}$ \\
\hline $\begin{array}{c}\mathrm{SaO}_{2} \\
\%\end{array}$ & $\begin{array}{c}\text { Controle } \\
\text { HES } \\
\text { SS }\end{array}$ & $\begin{array}{c}99 \pm 0,3 \\
98,7 \pm 1,1 \\
98,3 \pm 1,6\end{array}$ & $\begin{array}{c}99 \pm 0,1 \\
98,8 \pm 0,6 \\
97 \pm 3,7\end{array}$ & $\begin{array}{c}98,8 \pm 0,4 \\
99 \pm 0,5 \\
97 \pm 4,2\end{array}$ & $\begin{array}{l}98,7 \pm 0,6 \\
99,1 \pm 0,5 \\
97,8 \pm 2,2\end{array}$ & $\begin{array}{c}98,7 \pm 0,7 \\
99 \pm 0,6 \\
98,2 \pm 1,5\end{array}$ & $\begin{array}{c}98 \pm 1,7 \\
99 \pm 0,6 \\
97,8 \pm 2,3\end{array}$ & $\begin{array}{l}98,5 \pm 0,9 \\
98,8 \pm 0,8 \\
97,4 \pm 2,8\end{array}$ \\
\hline $\begin{array}{c}\mathrm{PvO}_{2} \\
\mathrm{mmHg}\end{array}$ & $\begin{array}{c}\text { Controle } \\
\text { HES } \\
\text { SS }\end{array}$ & $\begin{array}{c}47,2 \pm 3,6 \\
47,8 \pm 3,8 \\
49,4 \pm 4\end{array}$ & $\begin{array}{c}46,9 \pm 2 \\
46,5 \pm 4,4 \\
50,4 \pm 5,2\end{array}$ & $\begin{array}{c}45,8 \pm 2,9^{*} \\
45 \pm 3,1^{*} \\
48,5 \pm 3,9^{*}\end{array}$ & $\begin{array}{l}46,4 \pm 3,1^{*} \\
44,7 \pm 3,4^{*} \\
45,9 \pm 4,3^{*}\end{array}$ & $\begin{array}{c}45,7 \pm 2,3^{*} \\
44,9 \pm 3,5^{*} \\
46 \pm 2,6^{*}\end{array}$ & $\begin{array}{c}47,9 \pm 3,4 \\
47,9 \pm 2,2 \\
50,7 \pm 4\end{array}$ & $\begin{array}{l}45,1 \pm 5,8 \\
47,7 \pm 3,4 \\
48,9 \pm 4,2\end{array}$ \\
\hline $\begin{array}{c}\mathrm{SvO}_{2} \\
\%\end{array}$ & $\begin{array}{c}\text { Controle } \\
\text { HES } \\
\text { SS }\end{array}$ & $\begin{array}{l}78,7 \pm 4,6 \\
79,9 \pm 2,5 \\
78,8 \pm 5,1\end{array}$ & $\begin{array}{l}79,4 \pm 2,3 \\
77,2 \pm 3,9 \\
76,7 \pm 5,7\end{array}$ & $\begin{array}{c}76,2 \pm 5,1 \\
74,1 \pm 4,6^{* a} \\
73,3 \pm 6^{* a b}\end{array}$ & $\begin{array}{c}75,5 \pm 5,6 \\
73,9 \pm 5^{*} \\
71,3 \pm 6,6^{\star a b}\end{array}$ & $\begin{array}{c}76 \pm 3,5 \\
74,6 \pm 4,4^{\star a} \\
71,2 \pm 6^{\star a}\end{array}$ & $\begin{array}{c}76,8 \pm 3,6 \\
78,6 \pm 2,7 \\
78 \pm 6\end{array}$ & $\begin{array}{c}77,1 \pm 5 \\
79,5 \pm 2,5 \\
77 \pm 5,5^{\mathrm{ab}}\end{array}$ \\
\hline $\begin{array}{c}\mathrm{pH} \\
\text { Arterial }\end{array}$ & $\begin{array}{c}\text { Controle } \\
\text { HES } \\
\text { SS }\end{array}$ & $\begin{array}{l}7,408 \pm 0,03 \\
7,424 \pm 0,06 \\
7,390 \pm 0,03\end{array}$ & $\begin{array}{c}7,436 \pm 0,01 \\
7,395 \pm 0,05^{\mathrm{a}} \\
7,316 \pm 0,06^{\text {tab }}\end{array}$ & $\begin{array}{c}7,410 \pm 0,02 \\
7,372 \pm 0,06^{* a} \\
7,267 \pm 0,04^{* a b}\end{array}$ & $\begin{array}{c}7,406 \pm 0,02 \\
7,401 \pm 0,05 \\
7,320 \pm 0,05^{\text {tab }}\end{array}$ & $\begin{array}{c}7,431 \pm 0,02 \\
7,404 \pm 0,04 \\
7,331 \pm 0,04^{\text {ab }}\end{array}$ & $\begin{array}{c}7,422 \pm 0,06 \\
7,401 \pm 0,03 \\
7,339 \pm 0,04^{\text {*ab }}\end{array}$ & $\begin{array}{c}7,468 \pm 0,03 \\
7,424 \pm 0,04^{\mathrm{a}} \\
7,367 \pm 0,05^{\mathrm{ab}}\end{array}$ \\
\hline $\begin{array}{l}\mathrm{HCO}^{3-} \\
\mathrm{mmol} / \mathrm{L}\end{array}$ & $\begin{array}{c}\text { Controle } \\
\text { HES } \\
\text { SS }\end{array}$ & $\begin{array}{l}27 \pm 1,8 \\
27,3 \pm 3 \\
26 \pm 1,7\end{array}$ & $\begin{array}{c}28 \pm 2 \\
26,4 \pm 2,6^{\mathrm{a}} \\
22,9 \pm 2,3^{\star a b}\end{array}$ & $\begin{array}{c}27,3 \pm 2,3 \\
25,4 \pm 2,4^{\star a} \\
21,1 \pm 1,9^{* a b}\end{array}$ & $\begin{array}{c}27,8 \pm 2,4 \\
27 \pm 2,1 \\
23,4 \pm 2,1^{\star a b}\end{array}$ & $\begin{array}{c}28,2 \pm 1,9 \\
27,4 \pm 2,1 \\
24,5 \pm 2,1^{\star a b}\end{array}$ & $\begin{array}{c}28,4 \pm 1,9 \\
27,5 \pm 2,1^{a} \\
25,4 \pm 2^{\text {ab }}\end{array}$ & $\begin{array}{c}29,4 \pm 1,3 \\
28,6 \pm 1,4^{*} \\
26,8 \pm 1,6^{\text {ab }}\end{array}$ \\
\hline$\underset{\mathrm{mmol} / \mathrm{L}}{\mathrm{Na}^{+}}$ & $\begin{array}{c}\text { Controle } \\
\text { HES } \\
\text { SS }\end{array}$ & $\begin{array}{l}139 \pm 2 \\
138 \pm 3 \\
139 \pm 2\end{array}$ & $\begin{array}{c}137 \pm 3 \\
138 \pm 2 \\
141 \pm 3^{\mathrm{ab}}\end{array}$ & $\begin{array}{c}138 \pm 4 \\
138 \pm 2 \\
141 \pm 2^{\mathrm{ab}}\end{array}$ & $\begin{array}{l}138 \pm 4 \\
139 \pm 2 \\
139 \pm 2\end{array}$ & $\begin{array}{l}136 \pm 1 \\
139 \pm 2 \\
138 \pm 2^{\mathrm{a}}\end{array}$ & $\begin{array}{l}137 \pm 4 \\
139 \pm 2 \\
139 \pm 1^{\mathrm{a}}\end{array}$ & $\begin{array}{l}135 \pm 2 \\
139 \pm 2 \\
138 \pm 2^{\mathrm{a}}\end{array}$ \\
\hline $\begin{array}{c}\mathrm{K}^{+} \\
\mathrm{mmol} / \mathrm{L}\end{array}$ & $\begin{array}{c}\text { Controle } \\
\text { HES } \\
\text { SS }\end{array}$ & $\begin{array}{l}3,8 \pm 0,3 \\
3,7 \pm 0,3 \\
3,7 \pm 0,3\end{array}$ & $\begin{array}{c}3,9 \pm 0,2 \\
3,6 \pm 0,1^{\mathrm{a}} \\
3,3 \pm 0,3^{\mathrm{ab}}\end{array}$ & $\begin{array}{c}4 \pm 0,3 \\
3,6 \pm 0,2^{\mathrm{a}} \\
3,4 \pm 0,3^{\text {ab }}\end{array}$ & $\begin{array}{c}4,2 \pm 0,2 \\
4 \pm 0,2^{\star^{\mathrm{a}}} \\
3,7 \pm 0,2^{\mathrm{ab}}\end{array}$ & $\begin{array}{c}4,2 \pm 0,3 \\
4 \pm 0,3^{*} \\
4 \pm 0,3^{*}\end{array}$ & $\begin{array}{l}4,2 \pm 0,3 \\
3,9 \pm 0,4^{\mathrm{a}} \\
3,8 \pm 0,3^{\mathrm{a}}\end{array}$ & $\begin{array}{c}4,2 \pm 0,3 \\
4,1 \pm 0,3^{*} \\
4 \pm 0,3^{* a}\end{array}$ \\
\hline $\begin{array}{c}\mathrm{BE} \\
\mathrm{mmol} / \mathrm{L}\end{array}$ & $\begin{array}{c}\text { Controle } \\
\text { HES } \\
\text { SS }\end{array}$ & $\begin{array}{l}2,6 \pm 1,9 \\
3,1 \pm 3,7 \\
1,4 \pm 1,7\end{array}$ & $\begin{array}{c}4 \pm 1,6 \\
1,9 \pm 3,1^{\mathrm{a}} \\
-2,7 \pm 2,6^{\mathrm{ab}}\end{array}$ & $\begin{array}{c}3 \pm 2,2 \\
0,6 \pm 3,2^{\star a} \\
-5,2 \pm 2,7^{\star b}\end{array}$ & $\begin{array}{c}3,5 \pm 2,2 \\
2,5 \pm 2,6 \\
-2,1 \pm 2,7^{\star b}\end{array}$ & $\begin{array}{c}4,5 \pm 1,8 \\
3 \pm 2,1^{\mathrm{a}} \\
-0,7 \pm 2,7^{\star b}\end{array}$ & $\begin{array}{c}4,3 \pm 2,4 \\
2,9 \pm 2,1^{a} \\
0 \pm 2,4^{* b}\end{array}$ & $\begin{array}{c}6 \pm 1,1 \\
4,4 \pm 1,5^{\star a} \\
1,8 \pm 1,8^{b}\end{array}$ \\
\hline $\begin{array}{l}\text { Lactato } \\
\text { arterial } \\
\mathrm{mmol} / \mathrm{L}\end{array}$ & $\begin{array}{c}\text { Controle } \\
\text { HES } \\
\text { SS }\end{array}$ & $\begin{array}{c}1,13 \pm 0,36 \\
1,67 \pm 0,23 \\
1,9 \pm 0,26\end{array}$ & $\begin{array}{c}1,2 \pm 0,39 \\
1,53 \pm 0,33^{\text {ª }} \\
1,46 \pm 0,33^{\text {*a }}\end{array}$ & $\begin{array}{c}1,1 \pm 0,37 \\
1,39 \pm 0,24^{\star a} \\
1,3 \pm 0,35^{*}\end{array}$ & $\begin{array}{c}1,13 \pm 0,38 \\
1,3 \pm 0,37^{*} \\
1,31 \pm 0,42^{*}\end{array}$ & $\begin{array}{c}0,89 \pm 0,30 \\
1,21 \pm 0,32^{\text {*a }} \\
1,27 \pm 0,51^{\text {*a }}\end{array}$ & $\begin{array}{c}0,83 \pm 0,32 \\
1,24 \pm 0,32^{\star a} \\
1,11 \pm 0,37^{\star a}\end{array}$ & $\begin{array}{l}0,82 \pm 0,28 \\
1,09 \pm 0,15^{*} \\
0,94 \pm 0,38^{*}\end{array}$ \\
\hline
\end{tabular}

$\mathrm{PaO}_{2}$ : pressão parcial de oxigênio no sangue arterial; $\mathrm{SaO}_{2}$ : saturação de oxigênio no sangue arterial; $\mathrm{PvO}_{2}$ : pressão parcial de oxigênio no sangue venoso misto; $\mathrm{SvO}_{2}$ : saturação de oxigênio no sangue venoso misto; $\mathrm{HCO}^{3-}$ : bicarbonato; $\mathrm{Na}^{+}$: sódio sérico; $\mathrm{K}^{+}$: potássio sérico; $\mathrm{BE}$ : excesso de base; T0: valor basal, início hemodiluição; $\mathrm{T} 1$ : 30 minutos início hemodiluição; T2: fim hemodiluição; T3: 60 minutos após a hemodiluição; T4: 120 minutos pós hemodiluição, início retransfusão; T5: fim retransfusão; T6: 60 minutos pós retransfusão * $p<0,05$ diferente de T0; ${ }^{a} p<0,05$ diferente do grupo Controle; ${ }^{b} p<0,05$ diferente do grupo HES. 
Tabela 5 - Parâmetros de oxigenação dos animais do grupo Controle e submetidos à hemodiluição com amido hidroxietílico (HES) ou Solução salina 0,9\% (SS) (média \pm desvio padrão)

\begin{tabular}{|c|c|c|c|c|c|c|c|c|}
\hline & & & & Tempo & & & & \\
\hline Parâmetro & Grupo & TO & T1 & T2 & T3 & T4 & T5 & T6 \\
\hline$\underset{\text { g.dL }}{\mathrm{Hb}}$ & $\begin{array}{c}\text { Controle } \\
\text { HES } \\
\text { SS }\end{array}$ & $\begin{array}{c}9 \pm 0,9 \\
9,1 \pm 0,4 \\
8,9 \pm 0,7\end{array}$ & $\begin{array}{l}9,2 \pm 0,8 \\
6,2 \pm 0,2^{*} \\
6,4 \pm 0,4^{*}\end{array}$ & $\begin{array}{c}9,4 \pm 0,9 \\
4,8 \pm 0,3^{* a} \\
5 \pm 0,2^{* a}\end{array}$ & $\begin{array}{c}9,2 \pm 1,1 \\
5,6 \pm 0,7^{\star a} \\
6,6 \pm 0,9^{\star a b}\end{array}$ & $\begin{array}{c}9,5 \pm 1,3 \\
6,4 \pm 0,9^{\star a} \\
7,1 \pm 1,4^{\star a b}\end{array}$ & $\begin{array}{c}10,3 \pm 1 \\
7,3 \pm 0,5^{\star a} \\
7,5 \pm 1^{* a}\end{array}$ & $\begin{array}{c}10,4 \pm 0,9 \\
8,3 \pm 0,5 \\
8,2 \pm 0,9\end{array}$ \\
\hline$\underset{\mathrm{mL} \cdot \mathrm{dL}^{-1}}{\mathrm{CaO}_{2}}$ & $\begin{array}{c}\text { Controle } \\
\text { HES } \\
\text { SS }\end{array}$ & $\begin{array}{c}12,8 \pm 1,29 \\
12,83 \pm 0,55 \\
12,45 \pm 0,77\end{array}$ & $\begin{array}{l}13,05 \pm 1,16 \\
8,94 \pm 0,34^{\star a} \\
9,02 \pm 0,61^{\star a}\end{array}$ & $\begin{array}{l}13,21 \pm 1,28 \\
7,13 \pm 0,34^{\star a} \\
7,11 \pm 0,45^{\star a}\end{array}$ & $\begin{array}{c}13,02 \pm 1,53 \\
8,2 \pm 1,06^{* a} \\
9,35 \pm 1,03^{\star a b}\end{array}$ & $\begin{array}{c}13,42 \pm 1,78 \\
9,3 \pm 1,14^{\star a} \\
10,10 \pm 1,91^{\star a b}\end{array}$ & $\begin{array}{c}14,27 \pm 1,31 \\
10,47 \pm 0,72^{* a} \\
10,61 \pm 1,15^{\star a}\end{array}$ & $\begin{array}{c}14,52 \pm 1,35 \\
11,71 \pm 0,59^{* a} \\
11,36 \pm 0,98^{* a}\end{array}$ \\
\hline $\begin{array}{l}\mathrm{CvO}_{2} \\
\mathrm{~mL} \cdot \mathrm{dL}^{-1}\end{array}$ & $\begin{array}{c}\text { Controle } \\
\text { HES } \\
\text { SS }\end{array}$ & $\begin{array}{c}9,99 \pm 1,46 \\
10,15 \pm 0,45 \\
9,81 \pm 1,20\end{array}$ & $\begin{array}{l}10,26 \pm 1,10 \\
6,76 \pm 0,31^{* a} \\
6,93 \pm 0,49^{* a}\end{array}$ & $\begin{array}{l}10,03 \pm 1,58 \\
5,08 \pm 0,36^{\star a} \\
5,18 \pm 0,32^{\star a}\end{array}$ & $\begin{array}{c}9,79 \pm 1,67 \\
5,88 \pm 0,85^{\star a} \\
6,6 \pm 0,66^{\star a}\end{array}$ & $\begin{array}{l}10,14 \pm 1,56 \\
6,79 \pm 1,08^{* a} \\
7,12 \pm 1,42^{* a}\end{array}$ & $\begin{array}{l}11,02 \pm 1,16 \\
8,07 \pm 0,67^{\star a} \\
8,29 \pm 1,28^{\star a}\end{array}$ & $\begin{array}{l}11,19 \pm 1,29 \\
9,21 \pm 0,52^{\star a} \\
8,83 \pm 1,15^{\star} a\end{array}$ \\
\hline $\begin{array}{c}\mathrm{DO}_{2} \mathrm{I} \\
\mathrm{mL} \cdot \mathrm{min}^{-1} \cdot \mathrm{m}^{-2}\end{array}$ & $\begin{array}{c}\text { Controle } \\
\text { HES } \\
\text { SS }\end{array}$ & $\begin{array}{l}655 \pm 53 \\
707 \pm 74 \\
692 \pm 89\end{array}$ & $\begin{array}{c}597 \pm 59 \\
639 \pm 40 \\
641 \pm 128\end{array}$ & $\begin{array}{c}628 \pm 103 \\
616 \pm 69^{*} \\
517 \pm 75^{\star 2 a b}\end{array}$ & $\begin{array}{c}622 \pm 59 \\
572 \pm 108^{*} \\
547 \pm 89^{*}\end{array}$ & $\begin{array}{l}622 \pm 73 \\
619 \pm 84^{*} \\
537 \pm 77^{*}\end{array}$ & $\begin{array}{c}637 \pm 73 \\
727 \pm 98^{\mathrm{a}} \\
692 \pm 127^{\mathrm{a}}\end{array}$ & $\begin{array}{l}669 \pm 68 \\
749 \pm 91^{a} \\
746 \pm 131\end{array}$ \\
\hline $\begin{array}{c}\mathrm{VO}_{2} \mathrm{I} \\
\mathrm{mL} \cdot \mathrm{min}^{-1} \cdot \mathrm{m}^{-2}\end{array}$ & $\begin{array}{c}\text { Controle } \\
\text { HES } \\
\text { SS }\end{array}$ & $\begin{array}{l}135 \pm 19 \\
147 \pm 14 \\
135 \pm 11\end{array}$ & $\begin{array}{l}128 \pm 15 \\
156 \pm 26 \\
141 \pm 17\end{array}$ & $\begin{array}{c}141 \pm 22 \\
175 \pm 26^{*} \\
139 \pm 24^{*}\end{array}$ & $\begin{array}{c}144 \pm 20 \\
160 \pm 35^{\star} \\
157 \pm 28^{*}\end{array}$ & $\begin{array}{c}146 \pm 15 \\
167 \pm 23^{*} \\
159 \pm 27^{\star}\end{array}$ & $\begin{array}{c}143 \pm 15 \\
166 \pm 23^{*} \\
149 \pm 15^{*}\end{array}$ & $\begin{array}{c}151 \pm 16 \\
159 \pm 18^{*} \\
162 \pm 28^{*}\end{array}$ \\
\hline TEO2 \% & $\begin{array}{c}\text { Controle } \\
\text { HES } \\
\text { SS }\end{array}$ & $\begin{array}{l}21 \pm 3 \\
21 \pm 3 \\
21 \pm 5\end{array}$ & $\begin{array}{c}21 \pm 2 \\
24 \pm 3^{\star a} \\
23 \pm 4^{\star a}\end{array}$ & $\begin{array}{c}23 \pm 3 \\
29 \pm 4^{\star a} \\
27 \pm 4^{* a}\end{array}$ & $\begin{array}{c}23 \pm 3 \\
28 \pm 5^{\star a} \\
29 \pm 5^{\star a}\end{array}$ & $\begin{array}{c}24 \pm 3 \\
27 \pm 5^{\star a} \\
30 \pm 5^{\star a}\end{array}$ & $\begin{array}{l}23 \pm 3 \\
23 \pm 2 \\
22 \pm 6\end{array}$ & $\begin{array}{l}23 \pm 4 \\
21 \pm 3 \\
22 \pm 5\end{array}$ \\
\hline
\end{tabular}

$\mathrm{Hb}$ : Hemoglobina; $\mathrm{CaO}_{2}$ : Conteúdo arterial de oxigênio; $\mathrm{CvO}_{2}$ : Conteúdo venoso de oxigênio; $\mathrm{DO}_{2}$ l: Índice de transporte de oxigênio; $\mathrm{VO}_{2}$ I: Índice de consumo de oxigênio; $\mathrm{TEO}_{2}$ : taxa de extração; T0: valor basal, início hemodiluição; T1: 30 minutos início hemodiluição; T2: fim hemodiluição; T3: 60 minutos após a hemodiluição; T4: 120 minutos pós hemodiluição, início retransfusão; T5: fim retransfusão; T6: 60 minutos pós retransfusão ${ }^{*} p<0,05$ diferente de T0; ${ }^{a} p<0,05$ diferente do grupo Controle; ${ }^{b} p<0,05$ diferente do grupo HES. 
Tabela 6 - Parâmetros de ecocardiografia dos animais do grupo Controle e submetidos à hemodiluição com amido hidroxietílico (HES) ou Solução salina 0,9\% (SS) (média \pm desvio padrão)

\begin{tabular}{|c|c|c|c|c|c|c|c|c|}
\hline \multicolumn{9}{|c|}{ Tempo } \\
\hline Parâmetro & Grupo & Tо & T1 & T2 & T3 & $\mathrm{T} 4$ & T5 & T6 \\
\hline $\mathrm{EF} \%$ & $\begin{array}{c}\text { Controle } \\
\text { HES } \\
\text { SS }\end{array}$ & $\begin{array}{l}67,2 \pm 4,4 \\
70,9 \pm 3,3 \\
68,8 \pm 2,1\end{array}$ & $\begin{array}{c}66,5 \pm 5,5 \\
72,9 \pm 5^{\mathrm{a}} \\
70,9 \pm 5,3^{\mathrm{a}}\end{array}$ & $\begin{array}{l}65,7 \pm 4,7 \\
70,8 \pm 1,9^{\mathrm{a}} \\
70,1 \pm 4,8^{\mathrm{a}}\end{array}$ & $\begin{array}{l}65,3 \pm 3 \\
71 \pm 4,7^{\mathrm{a}} \\
69 \pm 4,2^{\mathrm{a}}\end{array}$ & $\begin{array}{c}64,9 \pm 3,5 \\
70,2 \pm 4^{\mathrm{a}} \\
65,3 \pm 8,1^{\mathrm{a}}\end{array}$ & $\begin{array}{c}65,6 \pm 4,8 \\
68,8 \pm 4,5^{\mathrm{a}} \\
68,7 \pm 5^{\mathrm{a}}\end{array}$ & $\begin{array}{l}65,8 \pm 3,2 \\
68,2 \pm 4,3^{\mathrm{a}} \\
70,2 \pm 5,3^{\mathrm{a}}\end{array}$ \\
\hline EDV mL & $\begin{array}{c}\text { Controle } \\
\text { HES } \\
\text { SS }\end{array}$ & $\begin{array}{c}42,45 \pm 7,2 \\
38,9 \pm 10,63 \\
39,35 \pm 8,54\end{array}$ & $\begin{array}{c}39,76 \pm 6,66 \\
39,89 \pm 10,03 \\
38,88 \pm 12,45\end{array}$ & $\begin{array}{c}39,61 \pm 7,94 \\
45,9 \pm 9,09^{* \mathrm{a}} \\
30,43 \pm 6,5^{\star \mathrm{b}}\end{array}$ & $\begin{array}{c}40,39 \pm 9,54 \\
39,64 \pm 12,47 \\
27,95 \pm 5,96^{\star a b}\end{array}$ & $\begin{array}{c}37,29 \pm 9,45 \\
38,3 \pm 14,28 \\
26,6 \pm 10,28^{* a b}\end{array}$ & $\begin{array}{c}34,54 \pm 9,98 \\
45,56 \pm 10,57^{\star a} \\
40,35 \pm 6,65^{\mathrm{ab}}\end{array}$ & $\begin{array}{c}36,76 \pm 10,52 \\
40,95 \pm 10,98 \\
39,95 \pm 7,07\end{array}$ \\
\hline ESV mL & $\begin{array}{c}\text { Controle } \\
\text { HES } \\
\text { SS }\end{array}$ & $\begin{array}{l}13,87 \pm 4,01 \\
11,46 \pm 4,06 \\
12,42 \pm 2,39\end{array}$ & $\begin{array}{c}13,52 \pm 4,3 \\
11,07 \pm 4,35^{\mathrm{a}} \\
10,7 \pm 3,09^{\mathrm{a}}\end{array}$ & $\begin{array}{c}13,85 \pm 4,52 \\
13,51 \pm 3,33^{*} \\
9,08 \pm 2,46^{* a b}\end{array}$ & $\begin{array}{c}14,45 \pm 4,67 \\
11,09 \pm 4,10^{\mathrm{a}} \\
8,51 \pm 1,82^{* a b}\end{array}$ & $\begin{array}{c}13,17 \pm 4,15 \\
11,18 \pm 4,05^{\mathrm{a}} \\
8,85 \pm 2,5^{\star a b}\end{array}$ & $\begin{array}{l}12,15 \pm 4,91 \\
13,96 \pm 2,99^{*} \\
11,95 \pm 2,39^{b}\end{array}$ & $\begin{array}{l}12,54 \pm 3,84 \\
12,73 \pm 3,12 \\
10,94 \pm 2,29\end{array}$ \\
\hline LVAD $\mathrm{cm}^{2}$ & $\begin{array}{c}\text { Controle } \\
\text { HES } \\
\text { SS }\end{array}$ & $\begin{array}{l}16,45 \pm 1,99 \\
15,53 \pm 2,83 \\
14,81 \pm 2,34\end{array}$ & $\begin{array}{c}16,21 \pm 1,62 \\
15,64 \pm 2,56 \\
14,7 \pm 2,91\end{array}$ & $\begin{array}{c}16,02 \pm 2,63 \\
16,47 \pm 3,75 \\
13,34 \pm 1,72^{\mathrm{ab}}\end{array}$ & $\begin{array}{c}15,55 \pm 2,05 \\
15,39 \pm 3,44 \\
12,25 \pm 1,61^{\star a b}\end{array}$ & $\begin{array}{c}14,52 \pm 2,09 \\
15,07 \pm 3,77 \\
12,59 \pm 2,29^{* a b}\end{array}$ & $\begin{array}{c}14,5 \pm 3,18 \\
17,24 \pm 2,77^{\star^{a}} \\
15,27 \pm 1,62^{\mathrm{b}}\end{array}$ & $\begin{array}{c}12,79 \pm 2,32 \\
15,86 \pm 2,93^{\mathrm{a}} \\
15,21 \pm 2,08^{\mathrm{a}}\end{array}$ \\
\hline LVAS $\mathrm{cm}^{2}$ & $\begin{array}{c}\text { Controle } \\
\text { HES } \\
\text { SS }\end{array}$ & $\begin{array}{l}7,88 \pm 1,31 \\
7,15 \pm 1,67 \\
6,83 \pm 1,19\end{array}$ & $\begin{array}{l}7,98 \pm 1,56 \\
6,83 \pm 1,89^{\mathrm{a}} \\
6,81 \pm 1,42^{\mathrm{a}}\end{array}$ & $\begin{array}{c}8,05 \pm 1,66 \\
7,83 \pm 1,41 \\
6,12 \pm 1,18^{\mathrm{ab}}\end{array}$ & $\begin{array}{c}8,19 \pm 1,5 \\
7,01 \pm 1,58^{\mathrm{a}} \\
5,82 \pm 1,03^{\star \mathrm{ab}}\end{array}$ & $\begin{array}{c}7,75 \pm 1,66 \\
6,99 \pm 1,66 \\
6,05 \pm 1,19^{\mathrm{ab}}\end{array}$ & $\begin{array}{c}7,34 \pm 2,03 \\
8,04 \pm 1,24^{*} \\
7,37 \pm 1,04\end{array}$ & $\begin{array}{r}7,49 \pm 1,4 \\
7,57 \pm 1,4 \\
7,04 \pm 0,97\end{array}$ \\
\hline $\begin{array}{l}\text { EF: Fraçã } \\
\text { esquerdo } \\
30 \text { minuto } \\
\text { pós hemo } \\
\text { diferente c }\end{array}$ & $\begin{array}{l}\text { ição, } \\
0 ;{ }^{a} p\end{array}$ & $\begin{array}{l}\text { o; EDV } \\
\text { LVAS:á } \\
\text { odiluiçã } \\
\text { cio retra } \\
, 05 \text { difer }\end{array}$ & $\begin{array}{l}\text { Dlume o } \\
\text { do vent } \\
\text { 2: fim h } \\
\text { usão; T } \\
\text { e do gru }\end{array}$ & $\begin{array}{l}\text { lico fina } \\
\text { lo esque } \\
\text { diluição } \\
\text { im retra } \\
\text { Controle }\end{array}$ & $\begin{array}{l}\text { V: Volum } \\
\text { m sístole } \\
60 \text { minutc } \\
\text { ão; T6: } 6 \\
0,05 \text { difere }\end{array}$ & $\begin{array}{l}\text { istólico fi } \\
\text { : valor ba } \\
\text { pós a her } \\
\text { hinutos pc } \\
\text { do grupo }\end{array}$ & $\begin{array}{l}\text { LVAD: áre } \\
\text { início hemo } \\
\text { tiluição; T4: } \\
\text { retransfusãc }\end{array}$ & $\begin{array}{l}\text { a ventrículc } \\
\text { diluição; T1 } \\
120 \text { minutos } \\
\text { * p < } 0,05\end{array}$ \\
\hline
\end{tabular}


Tabela 7 - Parâmetros hemodinâmicos dos animais do grupo Controle e submetidos à hemodiluição com amido hidroxietílico (HES) ou Solução salina 0,9\% (SS) (média \pm desvio padrão)

\begin{tabular}{|c|c|c|c|c|c|c|c|c|}
\hline \multicolumn{9}{|c|}{ Tempo } \\
\hline Parâmetro & Grupo & TO & T1 & T2 & T3 & T4 & T5 & T6 \\
\hline $\begin{array}{l}\text { ICEVAP } \\
\text { mL. } \mathrm{kg}^{-1}\end{array}$ & $\begin{array}{c}\text { Controle } \\
\text { HES } \\
\text { SS }\end{array}$ & $\begin{array}{c}9,1 \pm 1,1 \\
9,4 \pm 1 \\
10,2 \pm 1,4\end{array}$ & $\begin{array}{c}9,4 \pm 0,8 \\
9,7 \pm 1 \\
9,9 \pm 1,7\end{array}$ & $\begin{array}{c}9,5 \pm 1 \\
9,8 \pm 1,1 \\
10 \pm 1,7\end{array}$ & $\begin{array}{l}9,7 \pm 1,1 \\
9,9 \pm 1,1 \\
9,8 \pm 1,8\end{array}$ & $\begin{array}{c}10,1 \pm 1,5 \\
10 \pm 1 \\
9,8 \pm 1,7\end{array}$ & $\begin{array}{c}9,8 \pm 1,4 \\
10,2 \pm 1,6^{*} \\
10,7 \pm 2,1^{*}\end{array}$ & $\begin{array}{c}10,1 \pm 1,4 \\
10,2 \pm 1,6^{*} \\
10,8 \pm 2,5^{*}\end{array}$ \\
\hline $\begin{array}{l}\text { IVSIT } \\
\mathrm{mL} . \mathrm{m}^{-2}\end{array}$ & $\begin{array}{c}\text { Controle } \\
\text { HES } \\
\text { SS }\end{array}$ & $\begin{array}{l}865 \pm 76 \\
833 \pm 63 \\
822 \pm 83\end{array}$ & $\begin{array}{l}854 \pm 123 \\
899 \pm 67^{\mathrm{a}} \\
821 \pm 78^{\mathrm{b}}\end{array}$ & $\begin{array}{c}821 \pm 87 \\
947 \pm 85^{\star a} \\
775 \pm 37^{\text {ab }}\end{array}$ & $\begin{array}{c}818 \pm 104 \\
819 \pm 55 \\
720 \pm 61^{\text {*ab }}\end{array}$ & $\begin{array}{c}789 \pm 75 \\
799 \pm 60 \\
700 \pm 75^{\text {ab }}\end{array}$ & $\begin{array}{c}818 \pm 113 \\
878 \pm 83^{* a} \\
788 \pm 79^{b}\end{array}$ & $\begin{array}{c}757 \pm 72 \\
814 \pm 68^{\mathrm{a}} \\
781 \pm 72\end{array}$ \\
\hline $\mathrm{dp} \max$ & $\begin{array}{c}\text { Controle } \\
\text { HES } \\
\text { SS }\end{array}$ & $\begin{array}{l}290 \pm 59 \\
293 \pm 75 \\
293 \pm 24\end{array}$ & $\begin{array}{c}311 \pm 62 \\
333 \pm 79^{\star} \\
343 \pm 62^{*}\end{array}$ & $\begin{array}{c}324 \pm 60 \\
348 \pm 83^{*} \\
357 \pm 101^{*}\end{array}$ & $\begin{array}{c}336 \pm 60 \\
352 \pm 79^{*} \\
328 \pm 91^{*}\end{array}$ & $\begin{array}{c}353 \pm 67 \\
351 \pm 86^{*} \\
356 \pm 77^{*}\end{array}$ & $\begin{array}{c}349 \pm 97 \\
341 \pm 85^{*} \\
332 \pm 4^{*}\end{array}$ & $\begin{array}{c}357 \pm 100 \\
348 \pm 114^{*} \\
323 \pm 80^{*}\end{array}$ \\
\hline $\begin{array}{l}\text { ICEVAP: } \\
\text { intratoráci } \\
\text {; } 1: 30 \text { mi } \\
\text { minutos p } \\
<0,05 \text { dife }\end{array}$ & $\begin{array}{l}\text { dice cc } \\
\text { dp mas } \\
\text { os iníci } \\
\text { hemodi } \\
\text { hte de T }\end{array}$ & $\begin{array}{l}\text { eúdo es } \\
\text { ndice de } \\
\text { emodilu } \\
\text { ão, iníci }\end{array}$ & $\begin{array}{l}\text {; T2: } f \\
\text { transfi }\end{array}$ & $\begin{array}{l}\text { água } \\
\text { ventricula } \\
\text { modiluiçã } \\
\text { T5: fim re } \\
\text { rrupo Con }\end{array}$ & $\begin{array}{l}\text { monar; } \\
\text { squerda; } \\
\text { T3: } 60 \text { m } \\
\text { nsfusão; }\end{array}$ & $\begin{array}{l}\text { T: Índi } \\
\text { valor b } \\
\text { os após } \\
60 \text { min }\end{array}$ & $\begin{array}{l}\text { volume } \\
\text { início he } \\
\text { pós retra } \\
\text { podiluiç }\end{array}$ & $\begin{array}{l}\text { e sangue } \\
\text { nodiluição } \\
\mathrm{o} ; \mathrm{T} 4: 120 \\
\text { sfusão * p }\end{array}$ \\
\hline
\end{tabular}


Tabela 8 - Índices de reposição volêmica do grupo Controle e submetidos à hemodiluição com amido hidroxietílico (HES) ou Solução salina 0,9\% (SS) (média \pm desvio padrão)

\begin{tabular}{|c|c|c|c|c|c|c|c|c|}
\hline \multicolumn{9}{|c|}{ Tempo } \\
\hline Parâmetro & Grupo & TO & T1 & T2 & T3 & T4 & T5 & T6 \\
\hline$\underset{\text { L.min }}{\mathrm{IC}}$ & $\begin{array}{c}\text { Controle } \\
\text { HES } \\
\text { SS }\end{array}$ & $\begin{array}{c}5,32 \pm 0,39 \\
5,63 \pm 0,3 \\
5,46 \pm 0,43\end{array}$ & $\begin{array}{l}5,05 \pm 0,64 \\
6,9 \pm 0,28^{\star a} \\
6,9 \pm 0,96^{\star a}\end{array}$ & $\begin{array}{c}4,82 \pm 0,61 \\
8,14 \pm 0,63^{\star a} \\
6,99 \pm 0,78^{* a b}\end{array}$ & $\begin{array}{c}5,03 \pm 0,37 \\
6,65 \pm 0,57^{\star a} \\
5,87 \pm 0,92^{a b}\end{array}$ & $\begin{array}{c}4,95 \pm 0,43 \\
6,41 \pm 0,4^{\star a} \\
5,57 \pm 0,74^{\text {ab }}\end{array}$ & $\begin{array}{c}5,19 \pm 0,6 \\
6,81 \pm 0,6^{\star a} \\
6,59 \pm 0,91^{\star a}\end{array}$ & $\begin{array}{c}5,05 \pm 0,44 \\
6,27 \pm 0,64^{\star a} \\
6,37 \pm 0,84^{\star a}\end{array}$ \\
\hline$\triangle \mathrm{PP} \%$ & $\begin{array}{c}\text { Controle } \\
\text { HES } \\
\text { SS }\end{array}$ & $\begin{array}{l}9,1 \pm 3,1 \\
8,9 \pm 2,1 \\
9,3 \pm 1,5\end{array}$ & $\begin{array}{c}9,6 \pm 2,4 \\
7,6 \pm 1,6 \\
10,1 \pm 1,9\end{array}$ & $\begin{array}{c}10,6 \pm 3 \\
6,1 \pm 2,2^{\star a} \\
14,1 \pm 3,4^{\star a b}\end{array}$ & $\begin{array}{c}9,8 \pm 4 \\
8,3 \pm 2,5 \\
19,7 \pm 5,4^{* a b}\end{array}$ & $\begin{array}{c}10 \pm 3,4 \\
10,4 \pm 3 \\
18,9 \pm 4,5^{\star a b}\end{array}$ & $\begin{array}{c}11,3 \pm 2,9 \\
7,2 \pm 2^{\mathrm{a}} \\
9 \pm 2,5^{\mathrm{b}}\end{array}$ & $\begin{array}{c}10,4 \pm 2,5 \\
7,8 \pm 2,3^{\mathrm{a}} \\
9,6 \pm 2,7\end{array}$ \\
\hline $\begin{array}{l}\text { VPS } \\
\mathrm{mmHg}\end{array}$ & $\begin{array}{c}\text { Controle } \\
\text { HES } \\
\text { SS }\end{array}$ & $\begin{array}{c}4,9 \pm 2,1 \\
5,3 \pm 2 \\
5,6 \pm 1,3\end{array}$ & $\begin{array}{l}4,9 \pm 1,7 \\
4,5 \pm 1,7 \\
7,1 \pm 2^{* a b}\end{array}$ & $\begin{array}{c}5,3 \pm 1,7 \\
4,1 \pm 1,3 \\
9,7 \pm 4,6^{\star a b}\end{array}$ & $\begin{array}{c}5,5 \pm 2,2 \\
6 \pm 2,2 \\
11,5 \pm 3,4^{\star a b}\end{array}$ & $\begin{array}{c}5,9 \pm 2,2 \\
7,2 \pm 3^{*} \\
11,1 \pm 3,6^{\star a b}\end{array}$ & $\begin{array}{c}6,1 \pm 1,7 \\
4,3 \pm 1,6^{a} \\
6,1 \pm 2^{b}\end{array}$ & $\begin{array}{l}6,5 \pm 1,5 \\
5,3 \pm 2,2 \\
6,4 \pm 1,7\end{array}$ \\
\hline VVS \% & $\begin{array}{c}\text { Controle } \\
\text { HES } \\
\text { SS }\end{array}$ & $\begin{array}{c}9 \pm 1 \\
8 \pm 2 \\
10 \pm 3\end{array}$ & $\begin{array}{l}9 \pm 2 \\
8 \pm 2 \\
9 \pm 2\end{array}$ & $\begin{array}{c}10 \pm 3 \\
9 \pm 4 \\
12 \pm 3^{b}\end{array}$ & $\begin{array}{c}10 \pm 3 \\
12 \pm 3^{*} \\
16 \pm 4^{* a b}\end{array}$ & $\begin{array}{c}9 \pm 2 \\
12 \pm 4^{\star a} \\
16 \pm 4^{\star a b}\end{array}$ & $\begin{array}{c}10 \pm 3 \\
9 \pm 4 \\
10 \pm 4^{a}\end{array}$ & $\begin{array}{c}10 \pm 2 \\
12 \pm 5^{*} \\
14 \pm 8^{*}\end{array}$ \\
\hline $\begin{array}{c}\Delta \mathrm{up} \\
\mathrm{mmHg}\end{array}$ & $\begin{array}{c}\text { Controle } \\
\text { HES } \\
\text { SS }\end{array}$ & $\begin{array}{l}3,3 \pm 1,6 \\
2,8 \pm 0,8 \\
2,8 \pm 0,7\end{array}$ & $\begin{array}{l}2,9 \pm 0,9 \\
2,7 \pm 0,6 \\
3,1 \pm 0,8\end{array}$ & $\begin{array}{l}3,3 \pm 1,5 \\
2,1 \pm 0,5 \\
3,7 \pm 0,9\end{array}$ & $\begin{array}{l}3,6 \pm 1,9 \\
2,8 \pm 0,6 \\
4,5 \pm 2,1\end{array}$ & $\begin{array}{c}3,9 \pm 1,7 \\
3,1 \pm 0,5 \\
4 \pm 1,1\end{array}$ & $\begin{array}{c}4,2 \pm 1,5 \\
2,4 \pm 0,8 \\
3 \pm 0,7\end{array}$ & $\begin{array}{l}3,4 \pm 0,7 \\
2,6 \pm 0,8 \\
3,1 \pm 0,8\end{array}$ \\
\hline $\begin{array}{l}\Delta \text { down } \\
\mathrm{mmHg}\end{array}$ & $\begin{array}{c}\text { Controle } \\
\text { HES } \\
\text { SS }\end{array}$ & $\begin{array}{l}1,8 \pm 1,1 \\
2,5 \pm 1,6 \\
2,6 \pm 1,2\end{array}$ & $\begin{array}{l}2,1 \pm 0,7 \\
1,9 \pm 1,1 \\
4,1 \pm 2,1\end{array}$ & $\begin{array}{c}2,1 \pm 1 \\
2,1 \pm 1,4 \\
6 \pm 5,1^{*}\end{array}$ & $\begin{array}{c}2,1 \pm 0,8 \\
3,3 \pm 2 \\
6,9 \pm 2,6^{*}\end{array}$ & $\begin{array}{c}2 \pm 1,4 \\
4,1 \pm 3,1 \\
7 \pm 3,5^{*}\end{array}$ & $\begin{array}{c}2,2 \pm 1,1 \\
1,8 \pm 0,8 \\
3 \pm 1,6\end{array}$ & $\begin{array}{l}3,1 \pm 0,9 \\
2,7 \pm 1,8 \\
3,2 \pm 1,2\end{array}$ \\
\hline
\end{tabular}

IC: índice cardíaco pelo método de PiCCO ; $\triangle \mathrm{PP}$ : Variação da pressão de pulso; VPS: Variação da pressão sistólica; VVS: Variação do volume sistólico; $\Delta$ up: delta up; $\Delta$ down: delta down; T0: valor basal, início hemodiluição ;T1: 30 minutos início hemodiluição; T2: fim hemodiluição; T3: 60 minutos após a hemodiluição; T4: 120 minutos pós hemodiluição, início retransfusão; T5: fim retransfusão; T6: 60 minutos pós retransfusão * $p<0,05$ diferente de T0; ${ }^{a} p<0,05$ diferente do grupo Controle; ${ }^{b} p<0,05$ diferente do grupo HES. 


\section{Grupo Controle}

Todos os parâmetros avaliados não apresentaram alterações no decorrer do protocolo.

\section{Grupo HES}

Quanto ao grupo tratado com amido hidroxietílico (HES), verificou-se que os animais apresentaram alterações hemodinâmicas características decorrentes da hemodiluição.

Observou-se aumento não significativo $(p<0,05)$ da pressão venosa central, logo após o término da hemodiluição (T2), antes $(p>0,05)$ e depois da retransfusão $(p>0,05)$ (Tabela 3). Quanto à pressão de artéria pulmonar ocluída houve aumento significativo nos valores logo após a hemodiluição $(p<0,05)$

Em relação ao índice cardíaco, houve aumento significativo à partir da segunda metade da hemodiluição $(p<0,05)$ que perdurou em todos os momentos posteriores. Os diferentes métodos de avaliação do débito cardíaco, contínuo e por termodiluição, mostraram resultados semelhantes.

O índice de resistência vascular sistêmica foi significativamente menor, nos dois grupos intervenção, em todos os momentos após T0 em relação ao grupo controle $(p<0,05)$, no entanto sem diferença entre os grupos com reposição com amido hidroxietílico e solução salina a 0,9\%. Em relação ao índice de volume sistólico foi observado aumento logo após o 
início da hemodiluição (T1 e T2: p<0.05) voltando ao basal em T3 e T4 e se elevando novamente em T5 $(p<0,05)$ e voltando a valores próximos do basal em T6.

A pressão parcial de oxigênio no sangue venoso, assim como a saturação de oxigênio no sangue venoso misto diminuíram significativamente após a hemodiluição em relação ao momento T0 (T2:p<0,05, T3: $p<0,05$ e T4: $p<0,05)$ voltando a valores próximos ao basal após a retransfusão em T5 e T6. A saturação de oxigênio venoso medida de forma contínua apresentou valores próximos dos analisados pelo gasômetro.

O lactato sérico apresentou diminuição significativa em todos os momentos após T0 (T1: $p<0,05$, T2: $p<0,05$ eT3: $p<0,05$, T4: $p<0,05$, T5: $p<0,05$, T6: $p<0,05)$.

Quanto aos cálculos de oxigenação, a diminuição da hemoglobina resultou em diminuição significativa no conteúdo arterial e venoso de oxigênio ( $\mathrm{T} 1, \mathrm{~T} 2, \mathrm{~T} 3, \mathrm{~T} 4, \mathrm{~T} 5, \mathrm{~T} 6: \mathrm{P}<0,05)$, no índice de transporte de oxigênio do final da hemodiluição até a retransfusão $(T 2, T 3$ e T4: $p<0,05)$ e consequentemente, aumento compensatório da taxa de extração (T1, T2, T3 e T4: $p<0,05)$ até a retrasnfusão em T5 (Tabela 5).

A fração de ejeção na ecocardiografia transesofágica mostrou aumento em seus valores do grupo HES em comparação ao grupo controle em todos os momentos após o início da hemodiluição $(p<0,05)$. Assim como o volume diastólico final mensurado pela ecocardiografia que aumentou somente após a hemodiluição $(T 2 ; p<0,05)$ e a retransfusão $(T 5 ; p<0,05)$ voltando a valores basais nos momentos seguintes. 
A variação da pressão de pulso apresentou pequena diminuição logo após a hemodiluição T2 $(p<0,05)$. A variação da pressão sistólica apresentou aumento significativo antes da retransfusão em T4 $(p<0,05)$.

\section{Grupo Solução salina a 0,9\%}

Verificou-se diminuição significativa da pressão venosa central (T4: $p<0,05)$ duas horas após a hemodiluição e aumento significativo logo após a retransfusão (T5: $p<0,05)$. A pressão de artéria pulmonar ocluída não se alterou ao longo do tempo.

O índice cardíaco apresentou aumento significativo desde T1 $(p<0,05)$ até T3 $(p<0,05)$, voltando a valores basais em T4 e aumentando novamente após a retansfusão em T5 e T6 $(p<0,05)$.

O índice de resistência vascular sistêmica também diminuiu significativamente em todos os momentos $(p<0,05)$ exceto em T4 ( 2 horas após hemodiluição). Os diferentes métodos de avaliação do débito cardíaco, contínuo e por termodiluição, mostraram resultados semelhantes. Assim como entre os métodos de termodiluição transpulmonar e convencional.

O índice de trabalho do ventrículo direito apresentou aumento significativo em T5 $(p<0,05)$, após a retransfusão, em relação ao tempo basal T0.

Verificou-se diminuição significativa da pressão parcial de oxigênio no sangue arterial em T6 $(p<0,05)$. Houve diminuição no $\mathrm{pH}$ arterial, em todos os momentos $(p<0,05)$ exceto em T6 (1 hora após retransfusão) em relação 
ao valor basal. Houve queda significativa no bicarbonato arterial à partir de T1 até T5 $(p<0,05)$, quando comparado ao valor basal, observando uma recuperação progressiva. Quanto ao sangue venoso misto, houve diminuição significativa na pressão parcial de oxigênio e na saturação venosa mista nos momentos após a hemodiluição (T2: $p<0,05, T 3: p<0,05$ e T4: $p<0,05)$ em relação aos valores obtidos em T0, voltando a valores basais após a retransfusão. A saturação venosa mista contínua também apresentou diminuição de forma igual aos valores obtidos pela gasometria. O lactato arterial apresentou diminuição significativa $(p<0,05)$ em todos os momentos em relação aos valores basais.

Em relação aos parâmetros de oxigenação, houve diminuição significativa no conteúdo arterial e venoso $(p<0,05)$, em todos os momentos após a hemodiluição e aumento compensatório na taxa de extração do oxigênio $(p<0,05)$ até a retransfusão. $O$ índice de transporte de oxigênio, apresentou diminuição significativa em T2, T3 e T4 $(p<0,05)$.

A variação da pressão de pulso apresentou aumento siginificativo desde o final da hemodiluição, T2, até a retransfusão, T4 $(p<0,05)$. A variação da pressão sistólica apresentou comportamento semelhante, já com aumento significativo desde T1 até T4 $(p<0,05)$.

A fração de ejeção mensurada pela ecocardiografia transesofágica elevou-se em todos os momentos após a hemodiluição em relação ao grupo controle $(p<0,05)$. No entanto, o volume diastólico final também mensurado pela ecocardiografia apresentou diminuição em seus valores após T1 $(p<0,05)$ voltando a valores basais em T5 e T6. 


\section{Controle X HES x Solução salina a 0,9\%}

\section{Parâmetros hemodinâmicos}

\section{o Frequência cardíaca}

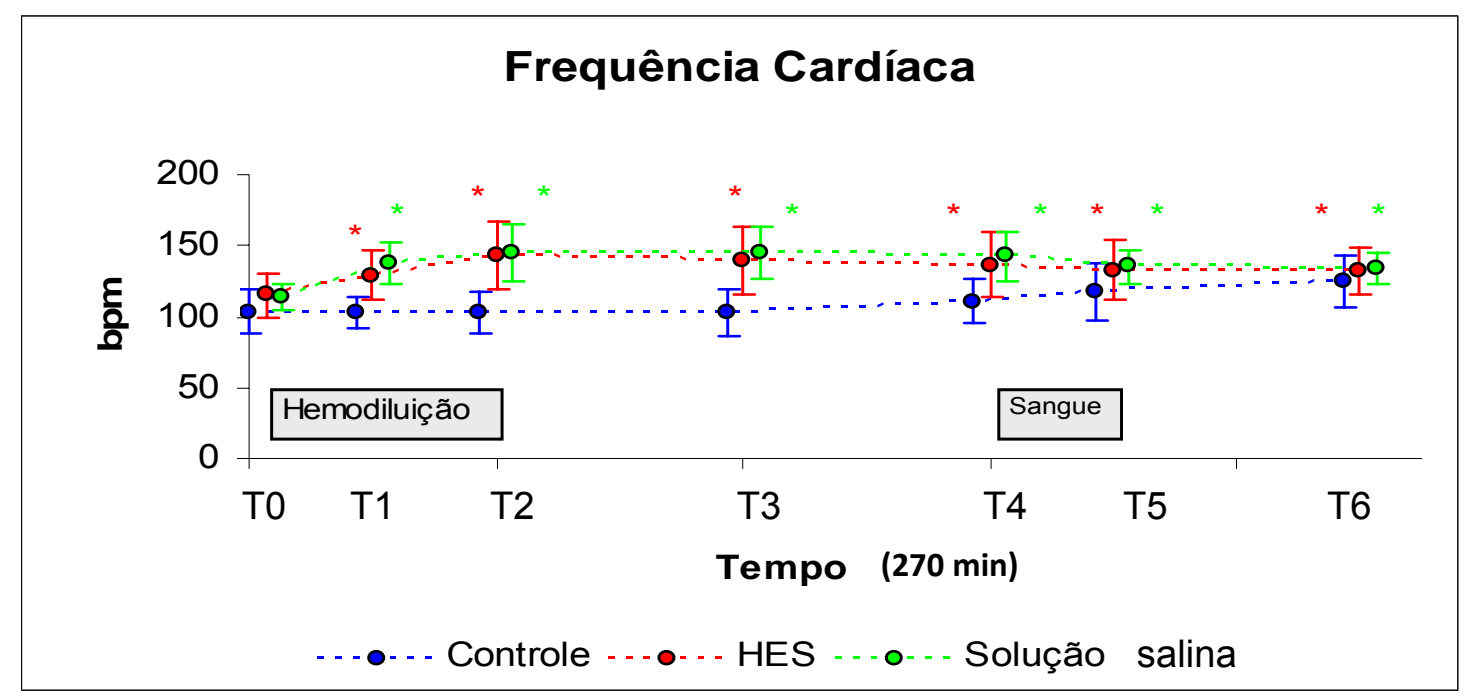

Figura 1 - Variação da frequência cardíaca (média e desvio padrão) em porcos submetidos à ventilação controlada (Grupo controle), hemodiluídos com amido hidroxietílico (Grupo HES) e hemodiluídos com Solução salina 0,9\% (Grupo SS). (*: p<0,05 diferente de T0) 


\section{o Pressão arterial}

Não foram observadas diferenças entre os grupos em relação à pressão arterial média.

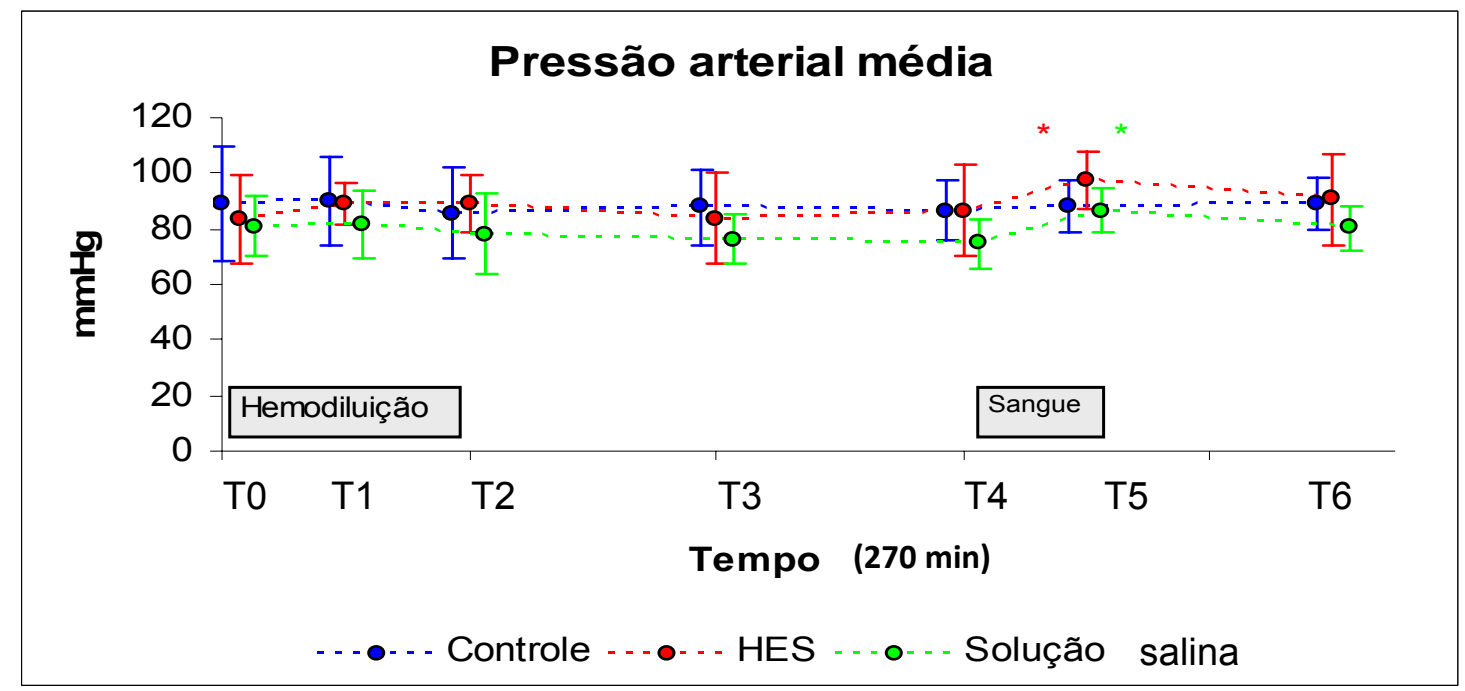

Figura 2 - Variação da pressão arterial média (média e desvio padrão) em porcos submetidos à ventilação controlada (Grupo controle), hemodiluídos com amido hidroxietílico (Grupo HES) e hemodiluídos com Solução salina $0,9 \%$ (Grupo SS). (*: $p<0,05$ diferente de T0) 


\section{o Pressão venosa central}

A pressão venosa central aumentou nos grupos submetidos à hemodiluição logo após a hemodiluição (T2) e após a retransfusão (T5), embora o aumento em T2 tenha sido significativo apenas no grupo HES após a hemodiluição, comparado ao controle. Entre os grupos hemodiluídos não houve diferença significativa.

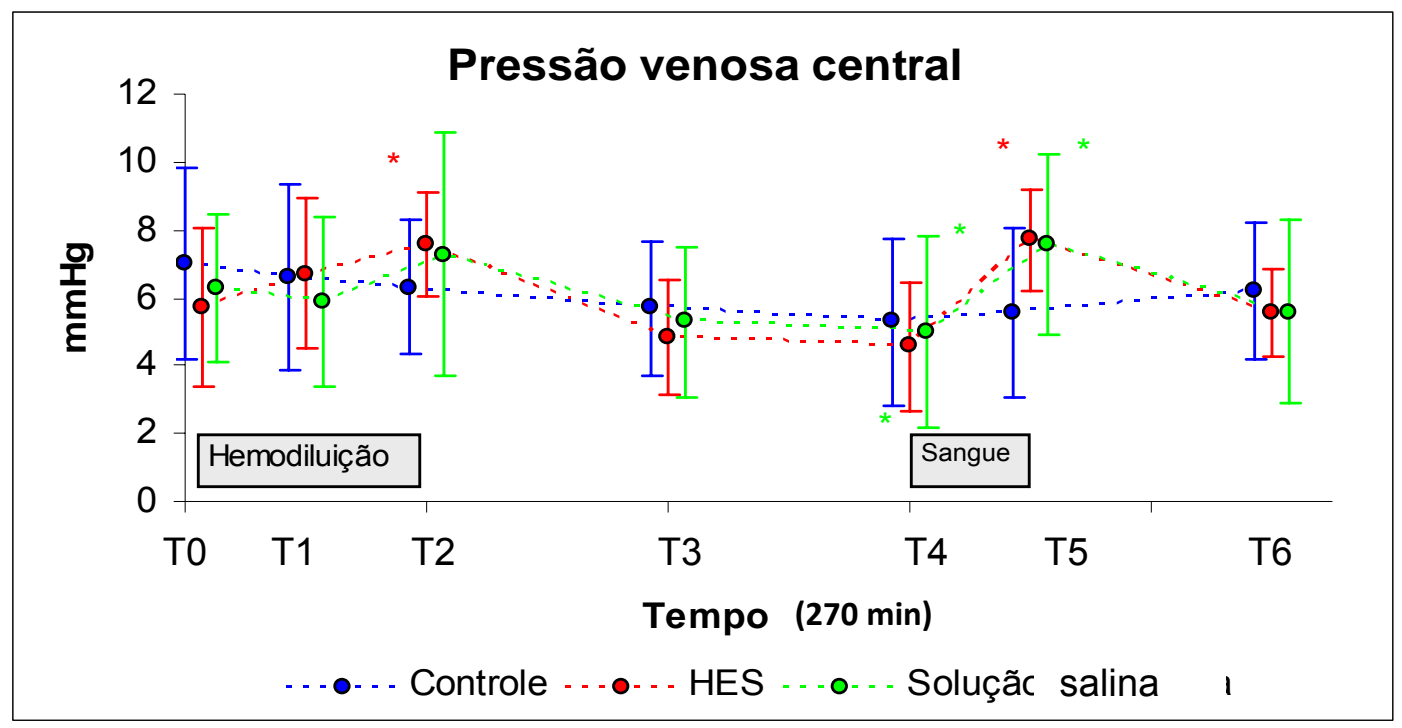

Figura 3 - Variação da pressão venosa central (média e desvio padrão) em porcos submetidos à ventilação controlada (Grupo controle), hemodiluídos com amido hidroxietílico (Grupo HES) e hemodiluídos com Solução salina $0,9 \%$ (Grupo SS). (*: $p<0,05$ diferente de T0) 
o Pressão de artéria pulmonar e pressão de oclusão da artéria pulmonar

Quanto à pressão de oclusão da artéria pulmonar, foi verificada diferença entre o grupo HES e o controle nos momentos T3 e T4 $(p<0,05)$ e entre os momentos T1, T2, T3 e T4 entre o grupo solução salina $0,9 \%$ e controle; entre os grupos hemodiluídos houve diferença apenas ao final da hemodiluição (T2) $(p<0,05)$ (Figura 5). Quanto à pressão de artéria pulmonar média, não foi observada diferença significativa entre os grupos, mas sim entre os momentos T5 e T6 $(p<0,05)$ com relação ao basal (Figura 4).

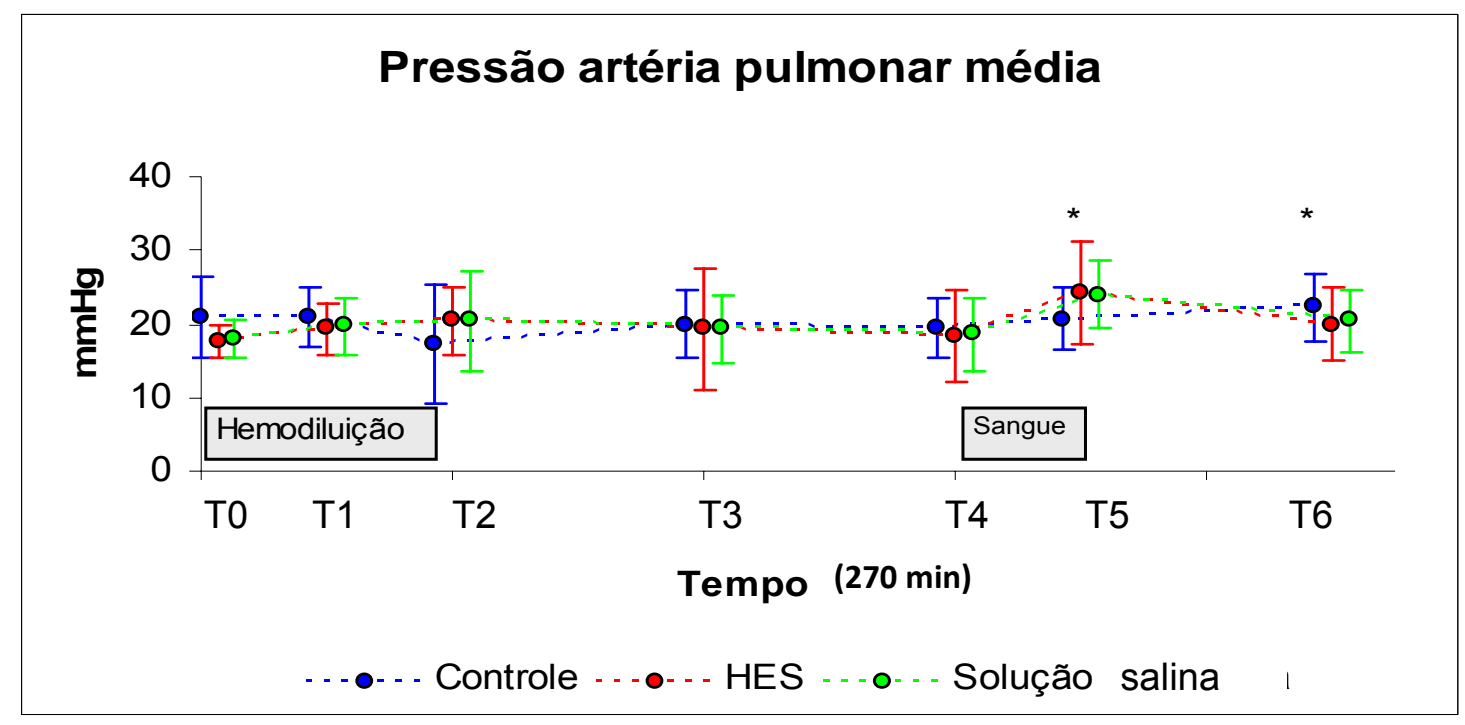

Figura 4 - Variação da pressão de artéria pulmonar média(média e desvio padrão) em porcos submetidos à ventilação controlada (Grupo controle), hemodiluídos com amido hidroxietílico (Grupo HES) e hemodiluídos com Solução salina $0,9 \%$ (Grupo SS). (*: $p<0,05$ diferente de TO) 


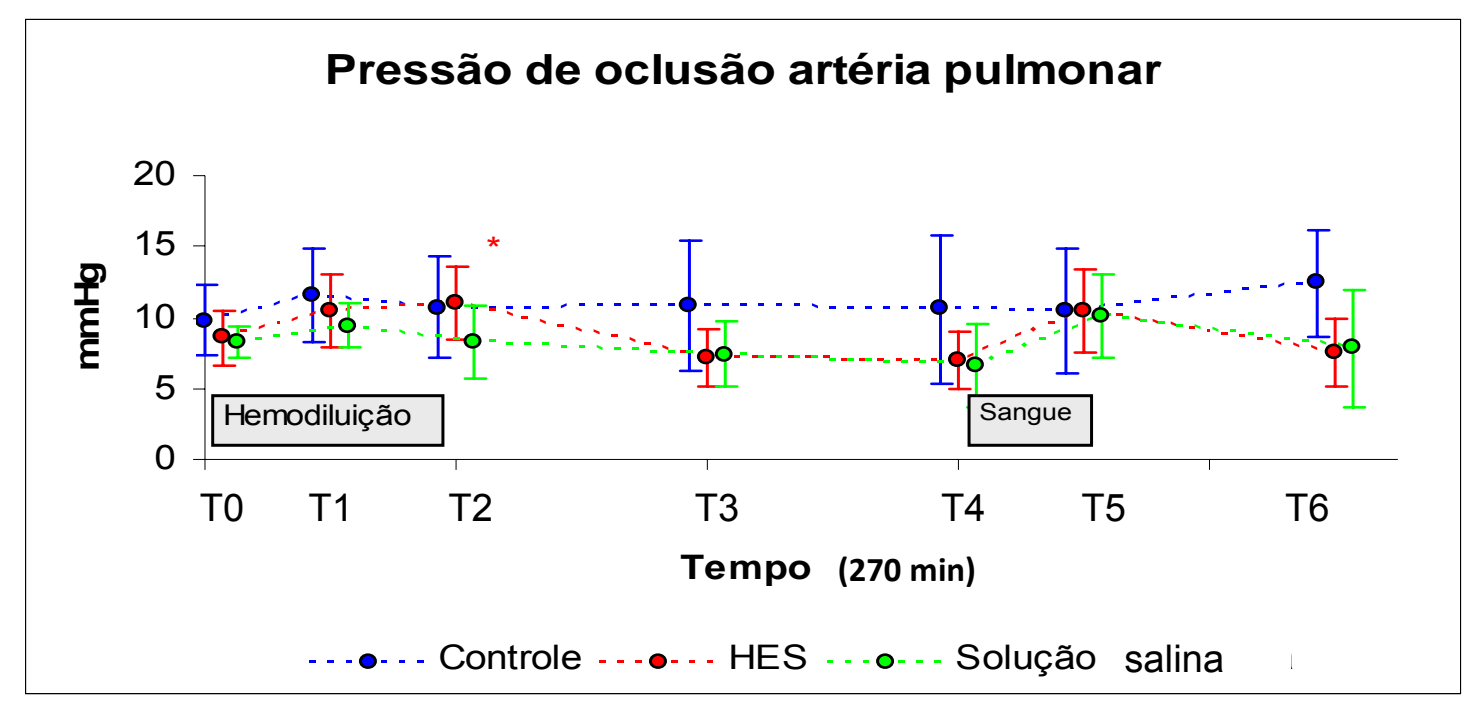

Figura 5 - Variação da pressão de oclusão da artéria pulmonar (média e desvio padrão) em porcos submetidos à ventilação controlada (Grupo controle), hemodiluídos com amido hidroxietílico (Grupo HES) e hemodiluídos com Solução salina 0,9\% (Grupo SS). (*: $\mathrm{p}<0,05$ diferente de T0). 


\section{o Índice cardíaco}

Em relação ao desempenho cardíaco, o índice cardíaco foi maior no grupo HES em relação ao grupo hemodiluído com a solução cristalóide em T2, T3 e T4 $(p<0,05)$ e maiores que o grupo controle em todos os momentos após T0. Os valores do grupo Solução salina foram maiores que o grupo controle em todos os momentos após T0 $(p<0,05)$ (Figura 6$)$.

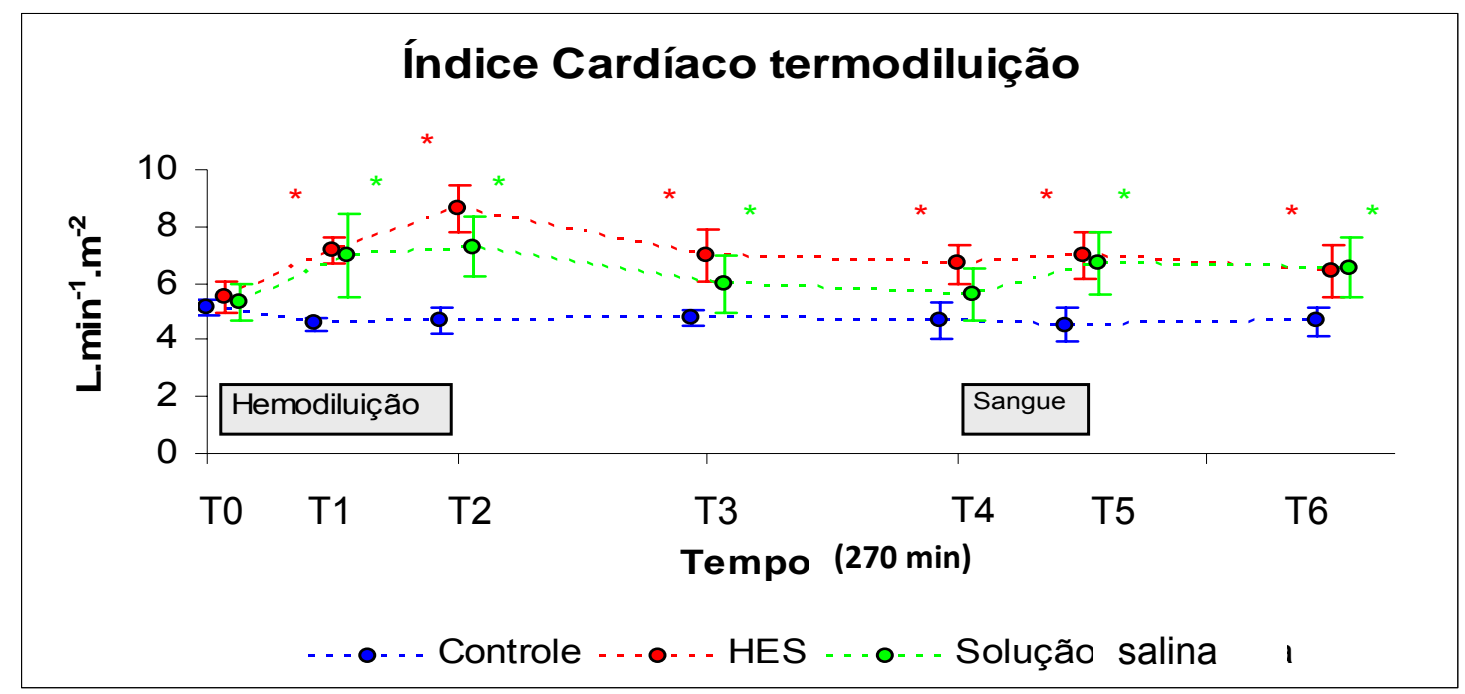

Figura 6 - Variação do índice cardíaco convencional (média e desvio padrão) em porcos submetidos à ventilação controlada (Grupo controle), hemodiluídos com amido hidroxietílico (Grupo HES) e hemodiluídos com Solução salina $0,9 \%$ (Grupo SS). (*: $\mathrm{p}<0,05$ diferente de T0) 


\section{o Índice de resistência vascular sistêmica}

O índice de resistência vascular sistêmica foi menor nos grupos HES e Solução salina em todos os momentos comparados ao grupo Controle, $(p<0,05)$ e sem diferença significativa entre os grupos intervenção. (Figura 7).

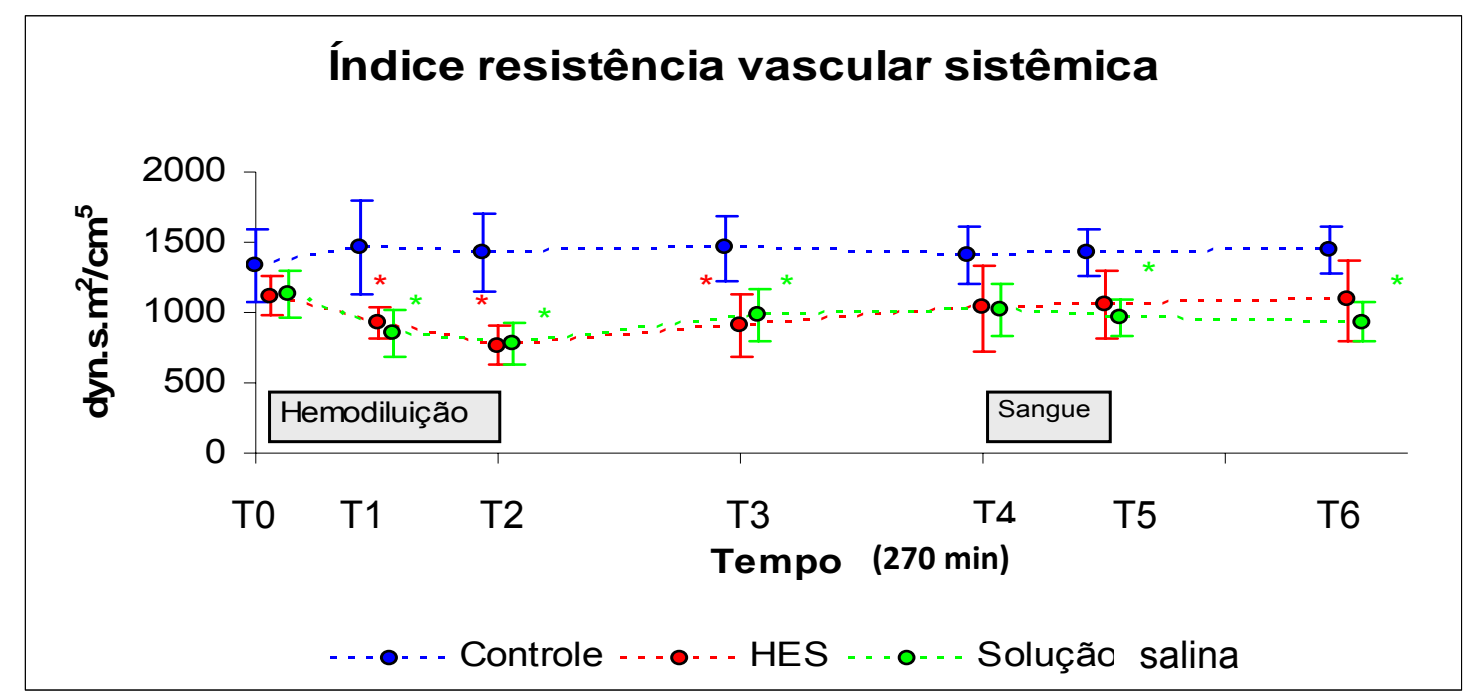

Figura 7 - Variação do índice de resistência vascular sistêmica (média e desvio padrão) em porcos submetidos à ventilação controlada (Grupo controle), hemodiluídos com amido hidroxietílico (Grupo HES) e hemodiluídos com Solução salina $0,9 \%$ (Grupo SS).(*: $p<0,05$ diferente de T0) 


\section{o Índice de resistência vascular pulmonar}

O índice de resistência vascular pulmonar foi menor que o controle no grupo HES nos momentos T1, T2, T3, T5 e T6 $(p<0,05)$ e o grupo solução salina menor que o controle nos momentos T1, T2, T5 e T6 $(p<0,05)$. Os grupos foram diferentes entre si nos momentos T2 e T4 $(p<0,05)$.

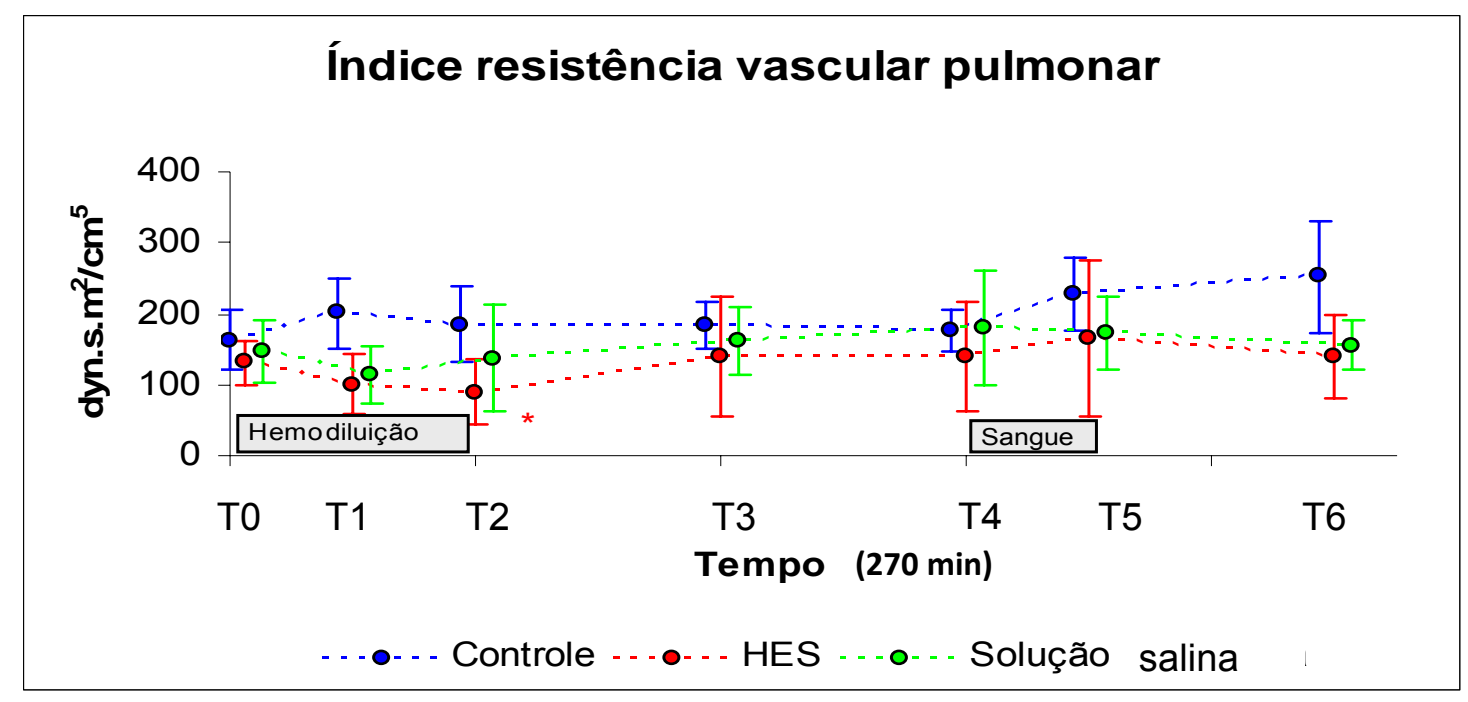

Figura 8 - Variação do índice de resistência vascular pulmonar (média e desvio padrão) em porcos submetidos à ventilação controlada (Grupo controle), hemodiluídos com amido hidroxietílico (Grupo HES) e hemodiluídos com Solução salina a $0,9 \%$ (Grupo SS). $\left({ }^{*}: p<0,05\right.$ diferente de T0) 


\section{o Índice de volume sistólico}

O índice de volume sistólico aumentou significativamente em todos os momentos após o início da hemodiluição no grupo HES $(p<0,05)$; no grupo solução salina esse aumento acontece nos momentos T1 e T2 $(p<0,05)$ voltando a valores basais em T3 e T4, voltando a se elevar em T5 e T6 $(p<0,05)$. Entre os grupos HES e solução salina há diferença significativa apenas nos momentos T2, T3 e T4 $(p<0,05)$, onde os valores do grupo solução salina são menores que os do grupo HES (Figura 9).

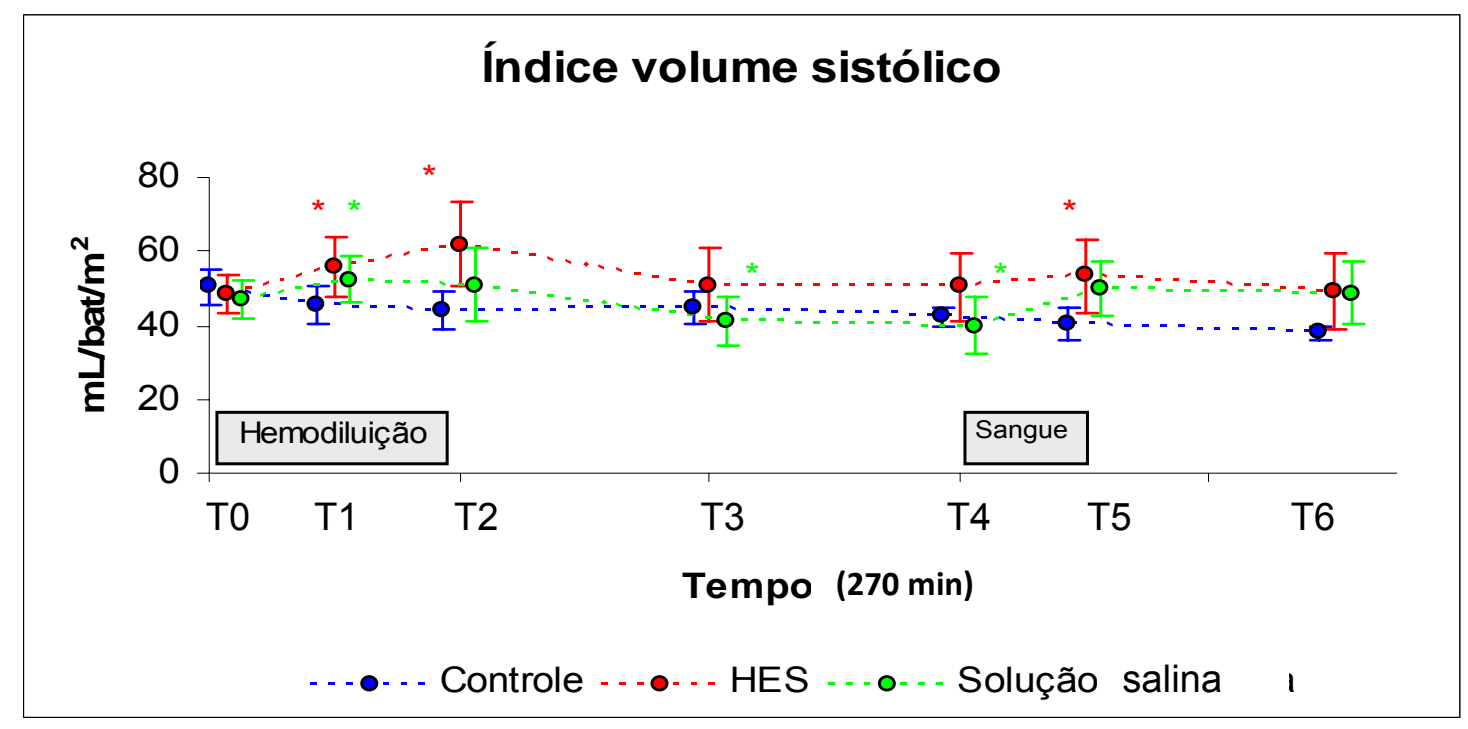

Figura 9 - Índice de volume sistólico (média e desvio padrão) em porcos submetidos à ventilação controlada (Grupo controle), hemodiluídos com amido hidroxietílico (Grupo HES) e hemodiluídos com Solução salina $0,9 \%$ (Grupo SS). (** $p<0,05$ diferente de T0) 


\section{Parâmetros de oxigenação}

\section{o Transporte de oxigênio}

Quanto à oferta de oxigênio (Figura 10), ambos os grupos que foram hemodiluídos apresentaram diminuição significativa em relação a T0 desde o final da hemodiluição T2 $(p<0,05)$ até o início da retransfusão T4. Apenas aqueles que receberam solução salina apresentaram diminuição significativa em relação ao grupo controle no momento logo ao final da hemodiluição $(T 2)(p<0,05)$.

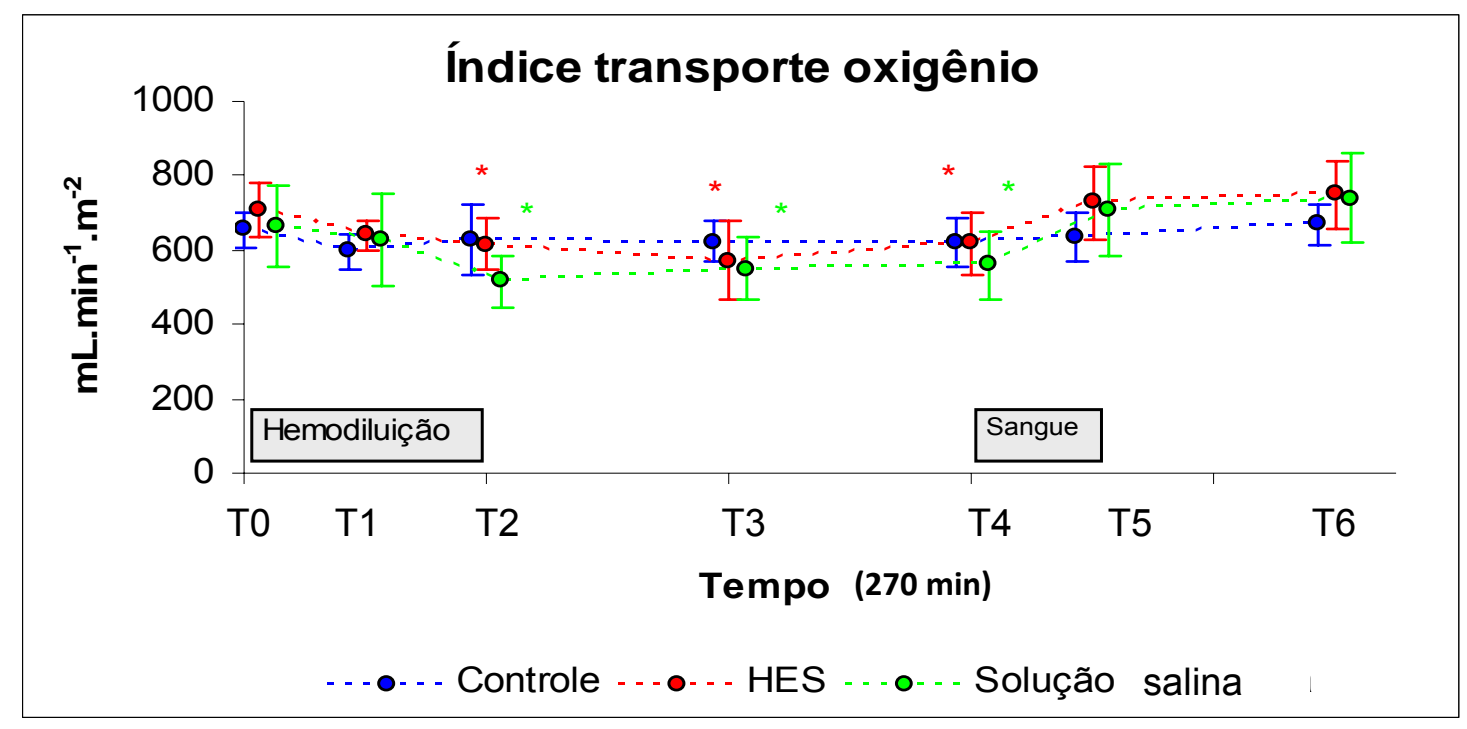

Figura 10 - Variação do índice de transporte de oxigênio (média e desvio padrão) em porcos submetidos à ventilação controlada (Grupo controle), hemodiluídos com amido hidroxietílico (Grupo HES) e hemodiluídos com Solução salina 0,9\% (Grupo SS). (*: $p<0,05$ diferente de T0) 


\section{o Consumo de oxigênio}

O consumo de oxigênio (Figura 11) apresentou aumento após a hemodiluição. Não houve diferença entre os grupos.

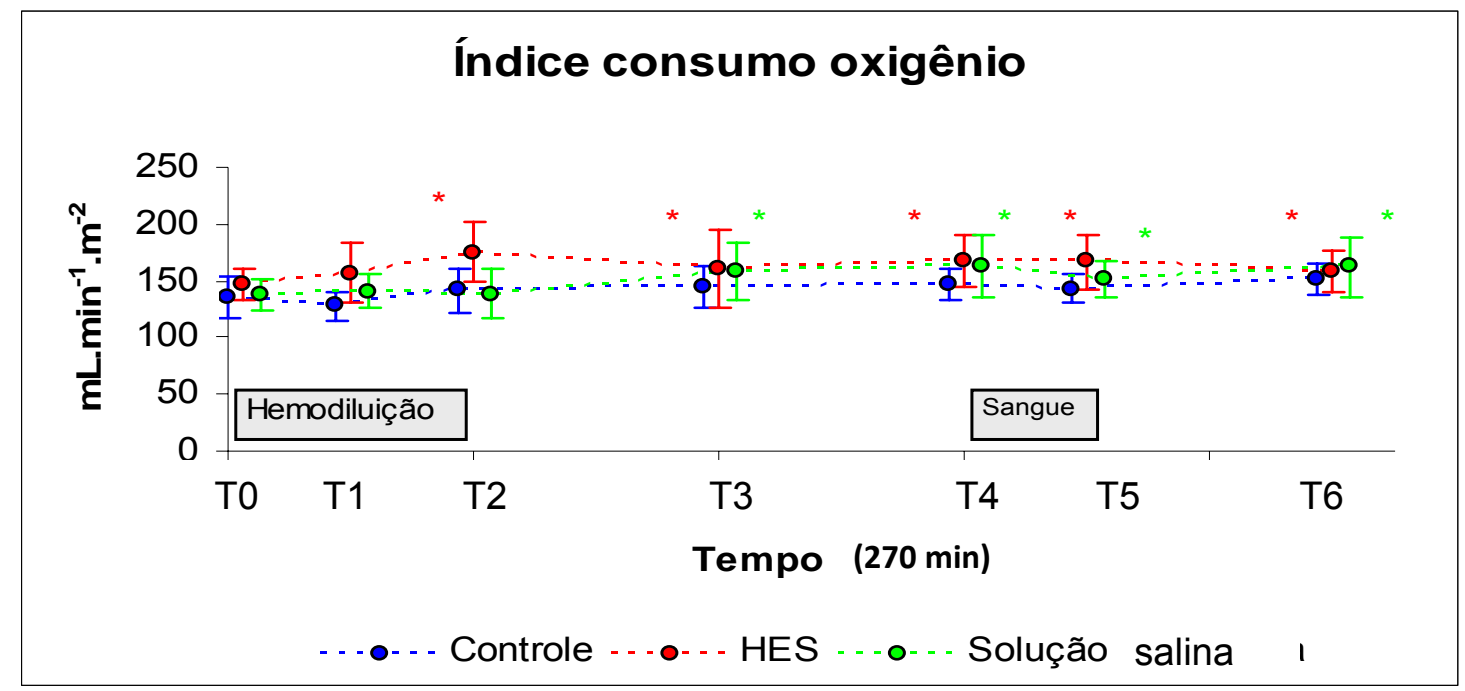

Figura 11 - Variação do índice de consumo de oxigênio (média e desvio padrão) em porcos submetidos à ventilação controlada (Grupo Controle), hemodiluídos com amido hidroxietílico (Grupo HES) e hemodiluídos com Solução salina 0,9\% (Grupo SS). $\left({ }^{*}: p<0,05\right.$ diferente de T0) 


\section{o Taxa de extração de oxigênio}

A taxa de extração de oxigênio elevou-se nos grupos submetidos à hemodiluição desde a metade do procedimento de hemodiluição (T1) até o início da retransfusão $(\mathrm{T} 4)(p<0,05)$, em relação ao grupo controle, voltando a valores basais após a retransfusão (T5)

(Figura 12).

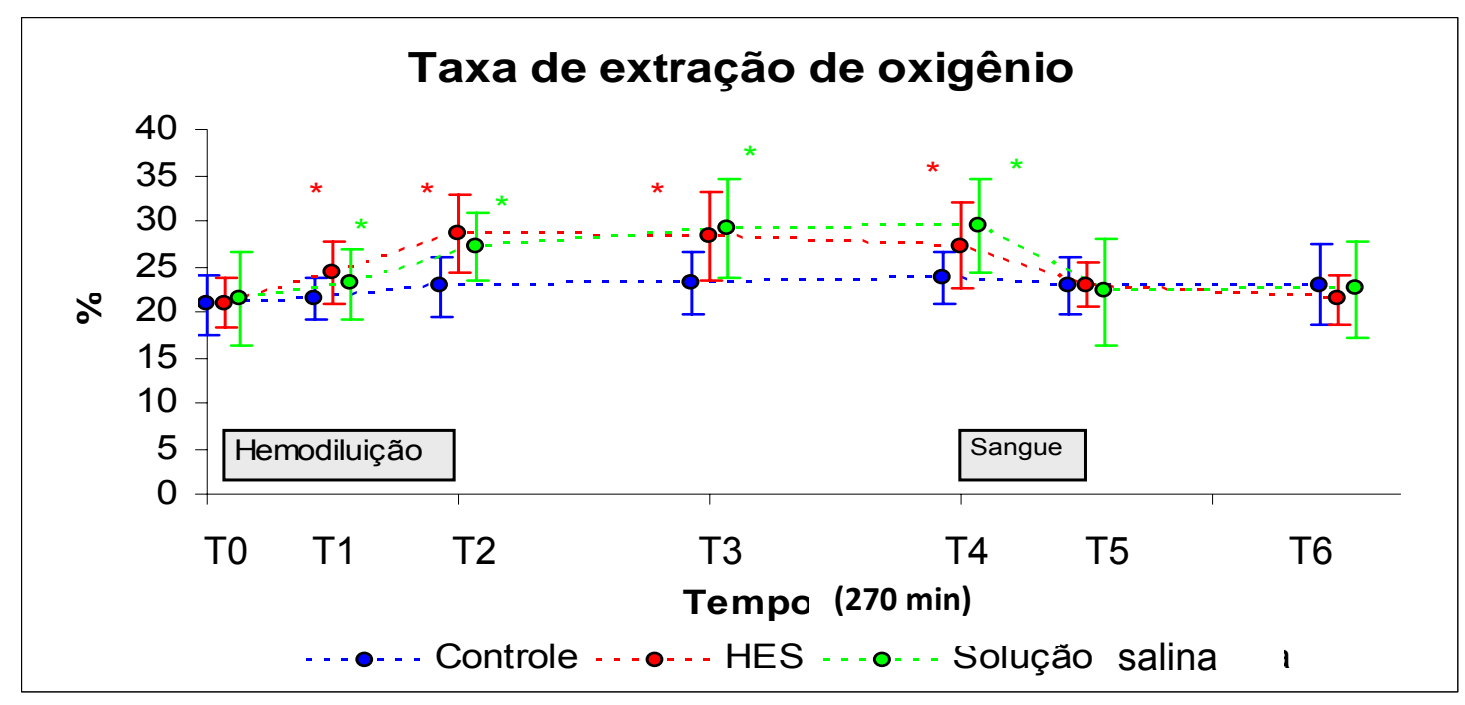

Figura 12 - Variação da taxa de extração (média e desvio padrão) em porcos submetidos à ventilação controlada (Grupo Controle), hemodiluídos com amido hidroxietílico (Grupo HES) e hemodiluídos com Solução salina $0,9 \%$ (Grupo SS). $\left({ }^{*}: p<\right.$ 0,05 diferente de T0) 


\section{o Hemogasometria}

Em relação aos dados da hemogasometria, $\mathrm{o} p \mathrm{pH}$ diminuiu significativamente no grupo que recebeu solução salina em todos os momentos após T0, em relação ao controle e ao grupo HES $(p<0,05)$ (Figura 13). Já o grupo HES apresentou diminuição nos momentos T1, T2 e T6 em relação ao controle $(p<0,05)$ (Figura 13).

A quantidade de sódio aumentou no grupo Solução salina 0,9\% em relação ao grupo controle nos momentos T1, T2, T4, T5 e T6 $(p<0,05)$ em relação ao grupo controle e nos momentos T1 e T2 em relação ao grupo HES $(p<0,05)$.

Os dois grupos hemodiluídos apresentam valores de saturação venosa mista menores que o grupo controle desde o final da hemodiluição (T2) até o final da retransfusão $(T 5)(p<0,05)$. A concentração de lactato arterial tanto no grupo HES como no grupo Solução salina elevou-se nos momentos T1, T2, T4 e T5 $(p<0,05)$ em relação ao controle, voltando a valores próximos do basal em T3 e T6, entre os dois grupos intervenção não há diferença estatística. 


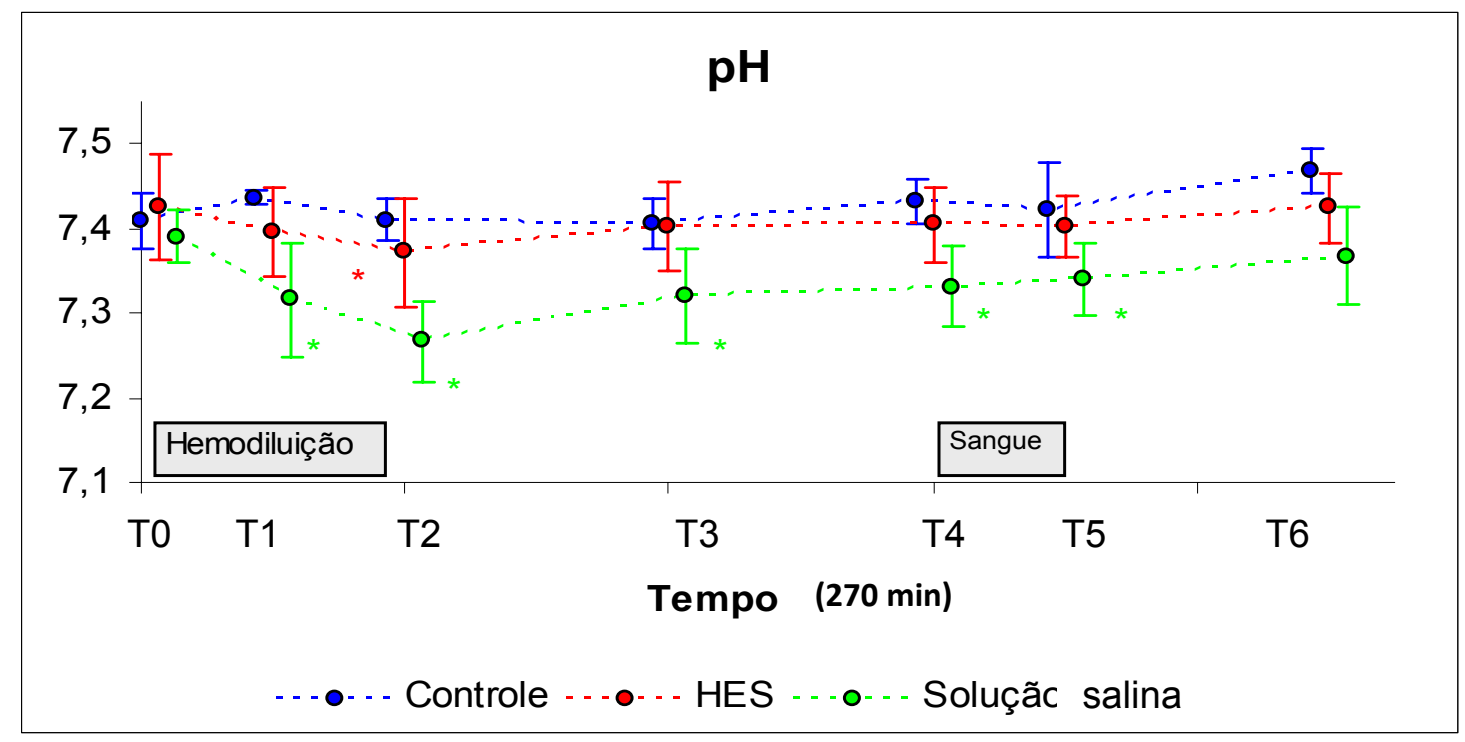

Figura 13 - Variação do pH (média e desvio padrão) em porcos submetidos à ventilação controlada (Grupo Controle), hemodiluídos com amido hidroxietílico (Grupo HES) e hemodiluídos com Solução salina $0,9 \%$ (Grupo SS). $\left({ }^{*}: p<0,05\right.$ diferente de T0) 


\section{o Responsividade aos fluídos}

Os valores de $\triangle P P$ do grupo HES apresentaram diferença estatisticamente significante com o grupo controle em T2, T5 e T6 $(p<0,05)$. Já o grupo Solução salina apresentou diferença significativa $(p<0,05)$ nos momentos T2, T3 e T4 em relação ao grupo controle. Entre os grupos intervenção há diferença entre os momentos T1, T2, T3 e T4 $(p<0,05)$ (Figura 14).

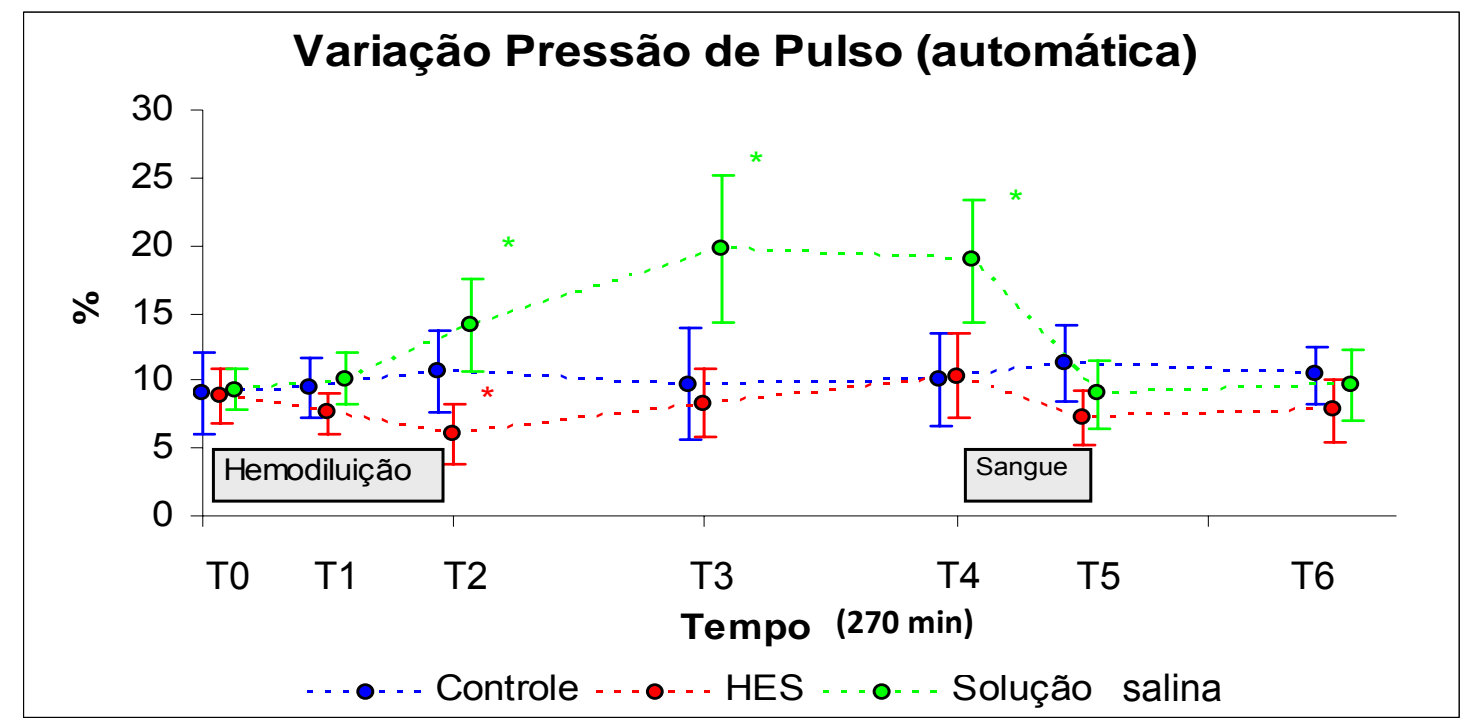

Figura 14 - Variação do $\triangle \mathrm{PP}$ (média e desvio padrão) em porcos submetidos à ventilação controlada (Grupo Controle), hemodiluídos com amido hidroxietílico (Grupo HES) e hemodiluídos com Solução salina $0,9 \%$ (Grupo SS). $\left({ }^{*}: p<0,05\right.$ diferente de T0)

Quanto à VPS, os valores do grupo HES são diferentes do grupo controle apenas em T5 $(p<0,05)$. O grupo Solução salina mostra diferença significativa nos momentos T1, T2, T3 e T4 $(p<0,05)$ em relação ao grupo controle. Entre os grupos intervenção há diferença $(p<0,05)$ entre os momentos T1, T2, T3, T4 e T5 $(p<0,05)$ (Figura 15). 


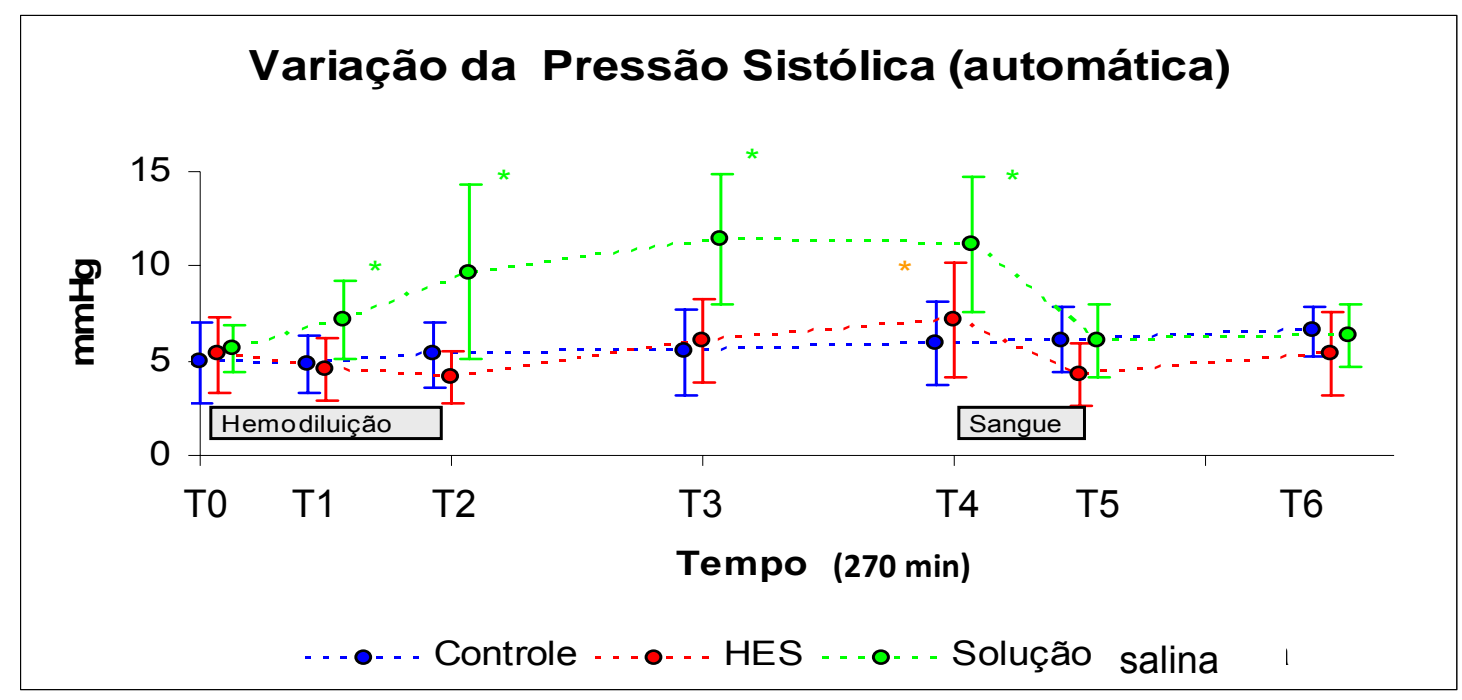

Figura 15 - Variação da VPS (média e desvio padrão) em porcos submetidos à ventilação controlada (Grupo Controle), hemodiluídos com amido hidroxietílico (Grupo HES) e hemodiluídos com Solução salina $0,9 \%$ (Grupo SS). $\left({ }^{*}: p<0,05\right.$ diferente de T0)

Os valores da VVS (Figura 16) são diferentes $(p<0,05)$ no grupo HES quando comparado ao grupo controle no momento T4, no início da retransfusão. No grupo Solução salina há diferença significativa nos momentos T3, T4 e T6 quando comparado ao grupo controle apesar de já haver aumento nos seus valores a partir de T2, mas sem diferença estatística, entre os grupos intervenção há diferença $(p<0,05)$ nos momentos T2, T3 e T4. 


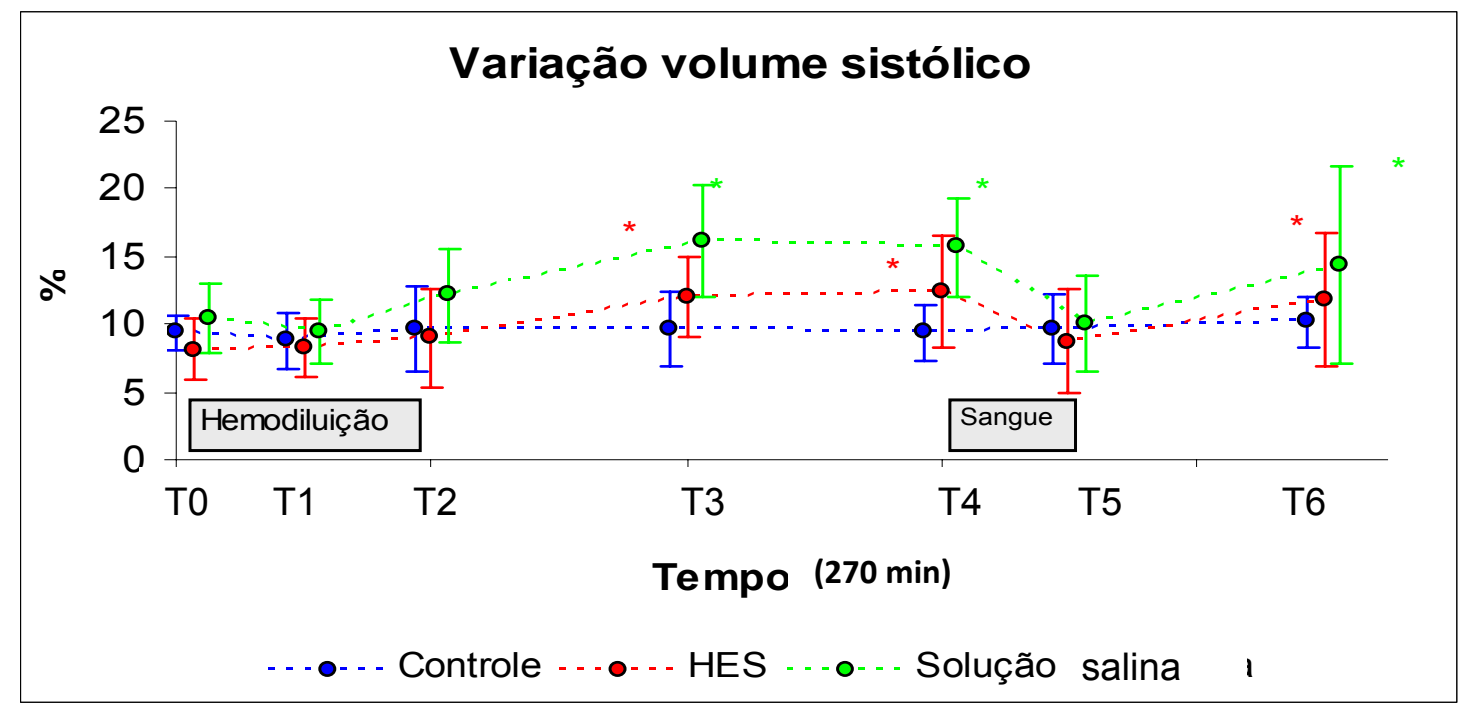

Figura 16 - Variação da VVS (média e desvio padrão) em porcos submetidos à ventilação controlada (Grupo Controle), hemodiluídos com amido hidroxietílico (Grupo HES) e hemodiluídos com Solução salina $0,9 \%$ (Grupo SS). $\left({ }^{*}: p<0,05\right.$ diferente de T0)

Os valores do índice de conteúdo de água extravascular pulmonar (ICEVAP) não tiveram diferença entre os três grupos, apenas diferença entre 0 valor basal nos momentos T5 e T6 $(p<0,05)$ (Figura 25). O índice do volume de sangue intratorácico (IVSIT) se mostrou maior no grupo HES quando comparado ao grupo controle nos momentos T1, T2, T5 e T6 $(p<0,05)$. Já o grupo Solução salina apresentou valores menores que o grupo controle nos momentos T2, T3 e T4 $(p<0,05)$. Os grupos hemodiluídos foram diferentes entre si desde T1 até T5 $(p<0,05)$ (Figura 17). 


\section{Índice água extravascular pulmonar}

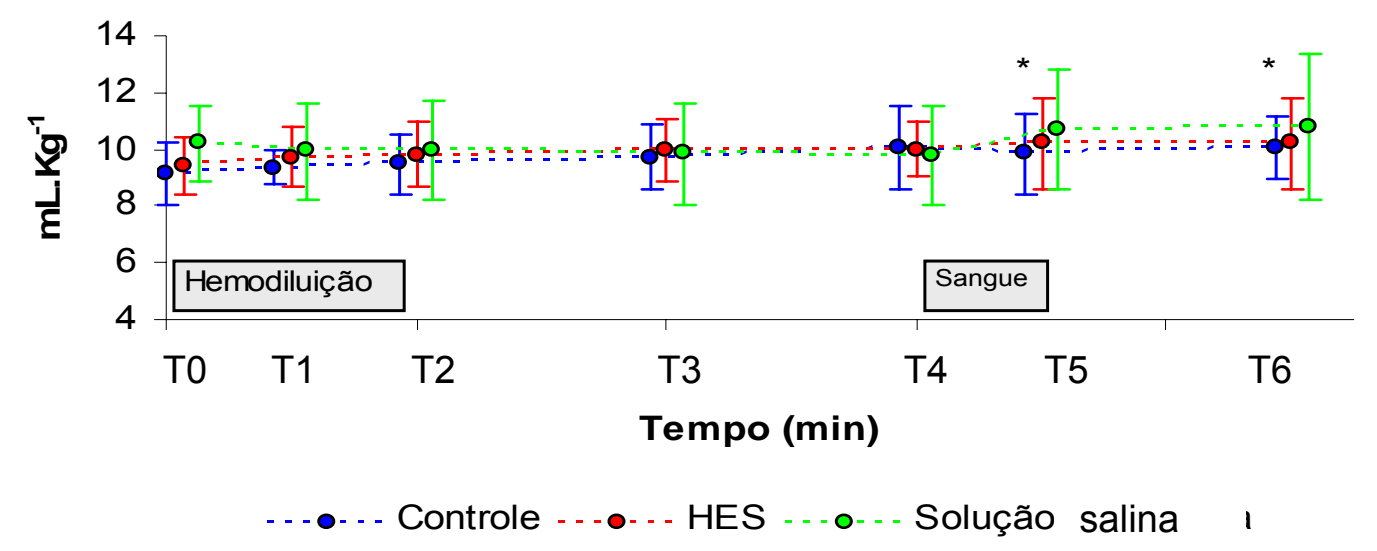

Figura 17 - Variação do índice de conteúdo de água extravascular pulmonar (média e desvio padrão) em porcos submetidos à ventilação controlada (Grupo Controle), hemodiluídos com amido hidroxietílico (Grupo HES) e hemodiluídos com Solução salina $0,9 \%$ (Grupo SS). ${ }^{*}: p<0,05$ diferente de T0)

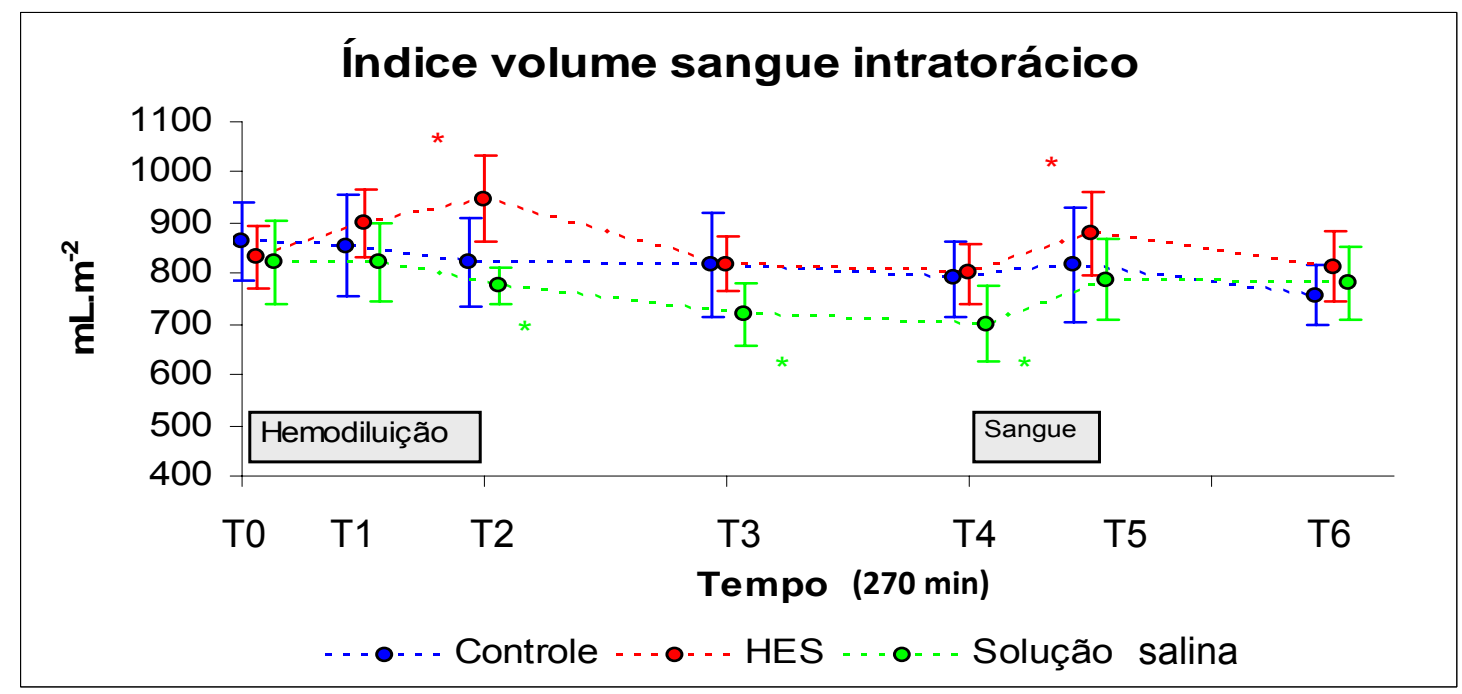

Figura 18 - Variação do índice de volume de sangue intratorácico (média e desvio padrão) em porcos submetidos à ventilação controlada (Grupo Controle), hemodiluídos com amido hidroxietílico (Grupo HES) e hemodiluídos com Solução salina $0,9 \%$ (Grupo SS). $\left({ }^{*}: p\right.$ $<0,05$ diferente de T0) 


\section{Ecocardiografia}

\section{o Fração de ejeção}

A fração de ejeção foi maior após T0 nos grupos hemodiluídos em relação ao grupo controle $(p<0,05)$. Não houve diferença entre os momentos nos três grupos. Não houve diferença entre o grupo HES e o grupo Solução salina.

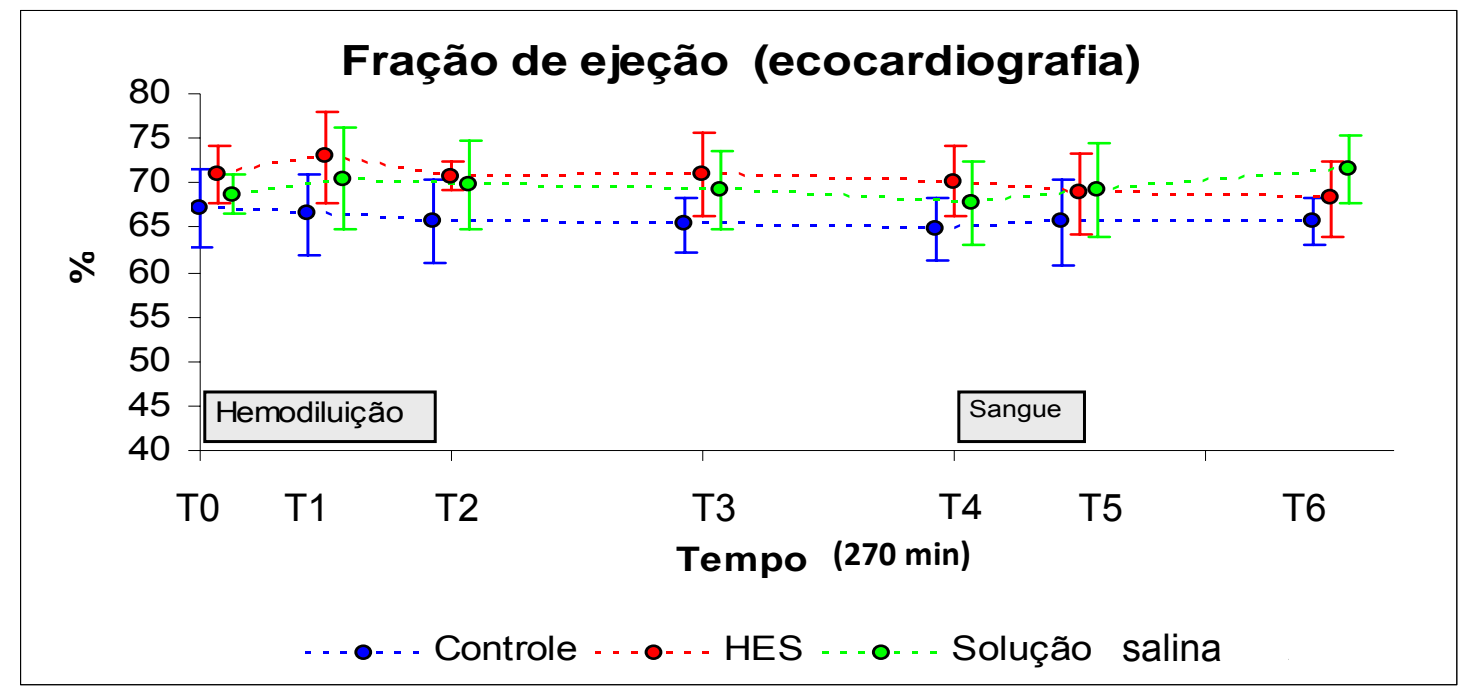

Figura 19 - Variação da fração de ejeção (média e desvio padrão) em porcos submetidos à ventilação controlada (Grupo Controle), hemodiluídos com amido hidroxietílico (Grupo HES) e hemodiluídos com Solução salina 0,9\% (Grupo SS). 


\section{o Volume diastólico final}

O volume diastólico final no grupo HES foi diferente do grupo controle logo ao final da hemodiluição (T2) e final da retransfusão (T5) $(p<0,05)$. O grupo Solução salina foi diferente do controle em todo o período entre o final da hemodiluição e final da retrasnfusão (T2, T3, T4 e T5) $(p<0,05)$. Os grupos hemodiluídos também foram diferentes entre o final da hemodiluição e o final da retransfusão $(p<0,05)$.

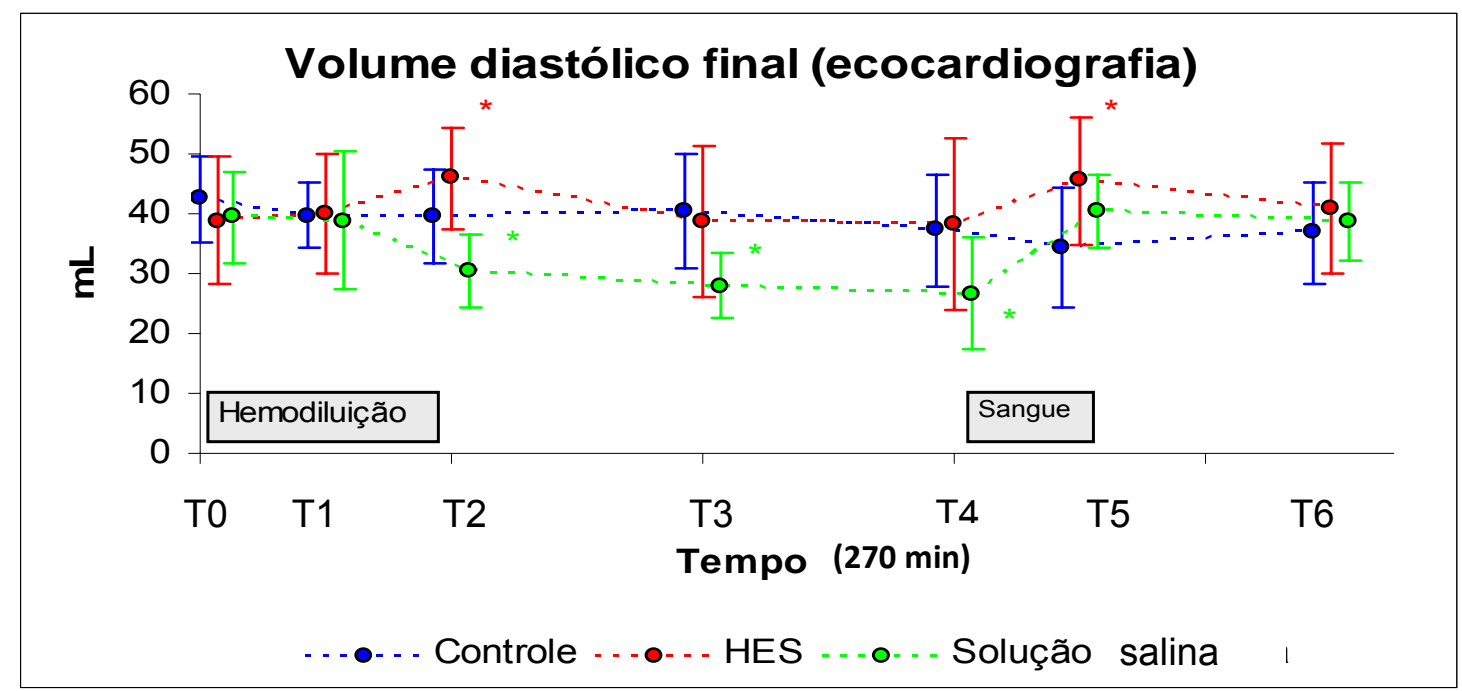

Figura 20 - Variação do volume diastólico final (média e desvio padrão) em porcos submetidos à ventilação controlada (Grupo Controle), hemodiluídos com amido hidroxietílico (Grupo HES) e hemodiluídos com Solução salina 0,9\% (Grupo SS). ( ${ }^{*}$ : $p<0,05$ diferente de T0) 


\section{Correlação}

As figuras a seguir mostram a dispersão dos valores dos parâmetros dos sete animais de cada grupo em estudo e a correlação das variáveis estáticas (PVC, PAP, POAP) com o $\triangle \mathrm{PP}$.

O $\triangle$ PP não apresentou boa correlação com nenhum dos parâmetros estáticos estudados, em nenhum dos 3 grupos.

\section{o Controle}

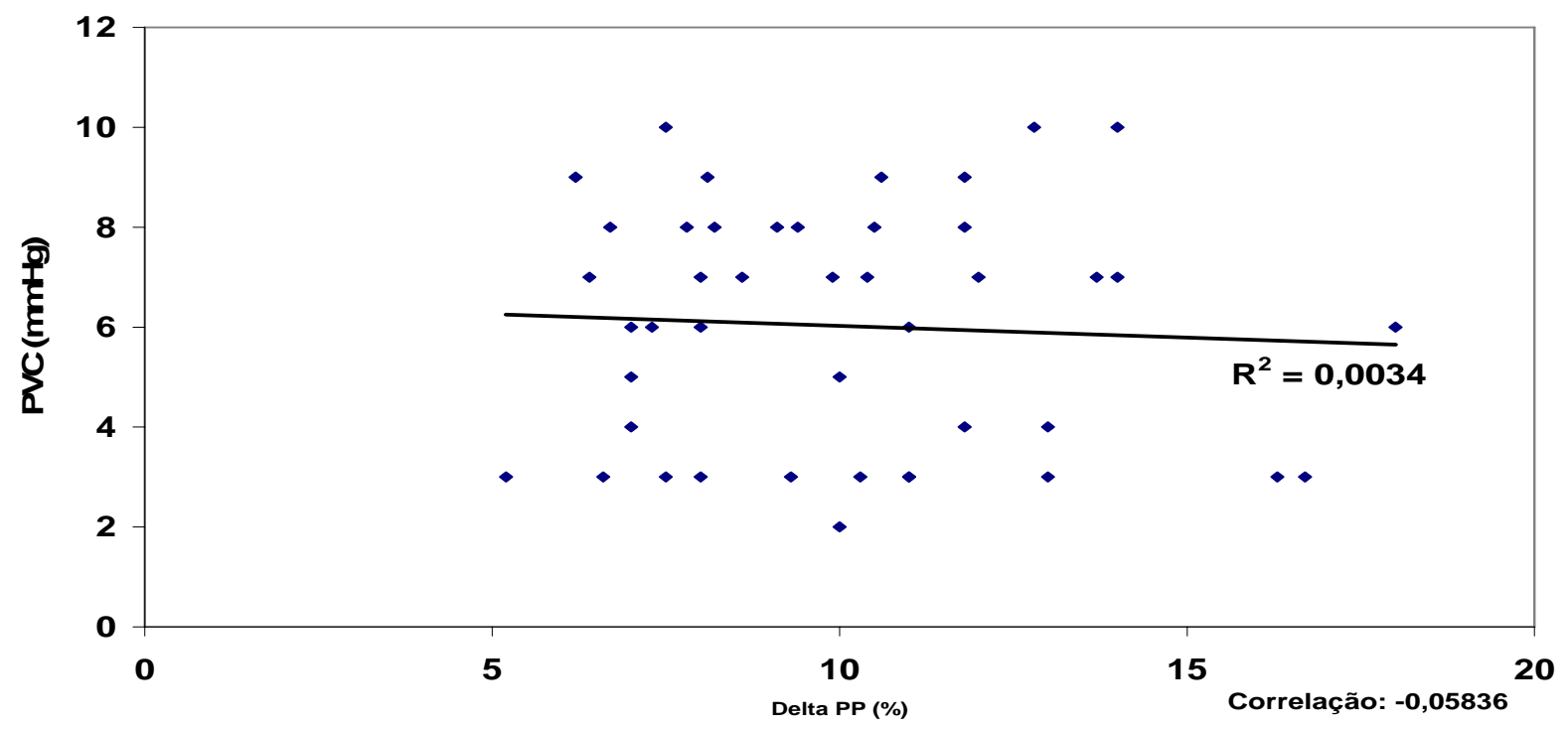

Figura 21 - Diagrama de dispersão da variação do Delta PP e PVC em porcos submetidos à ventilação controlada no grupo controle. 


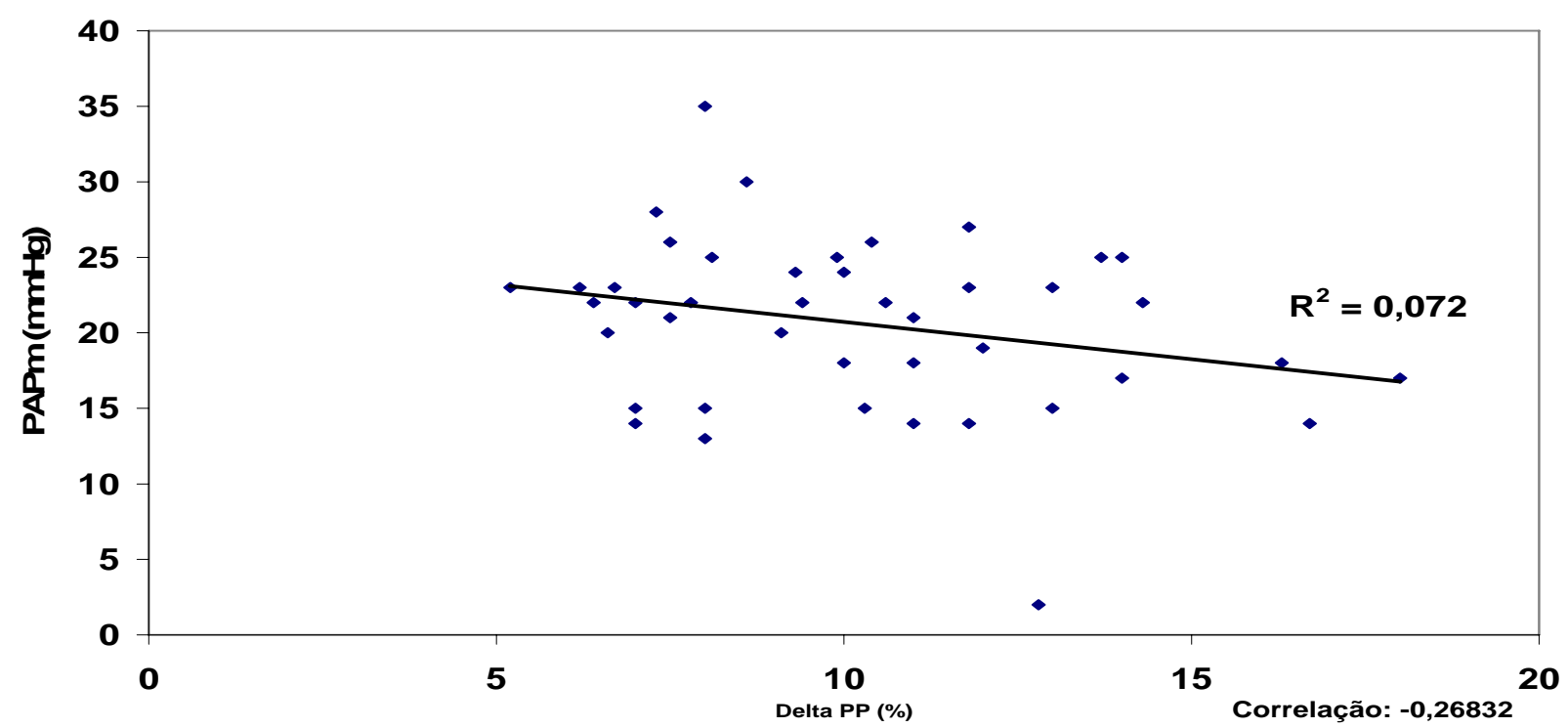

Figura 22 - Diagrama de dispersão da variação do Delta PP e PAPm em porcos submetidos à ventilação controlada no grupo controle.

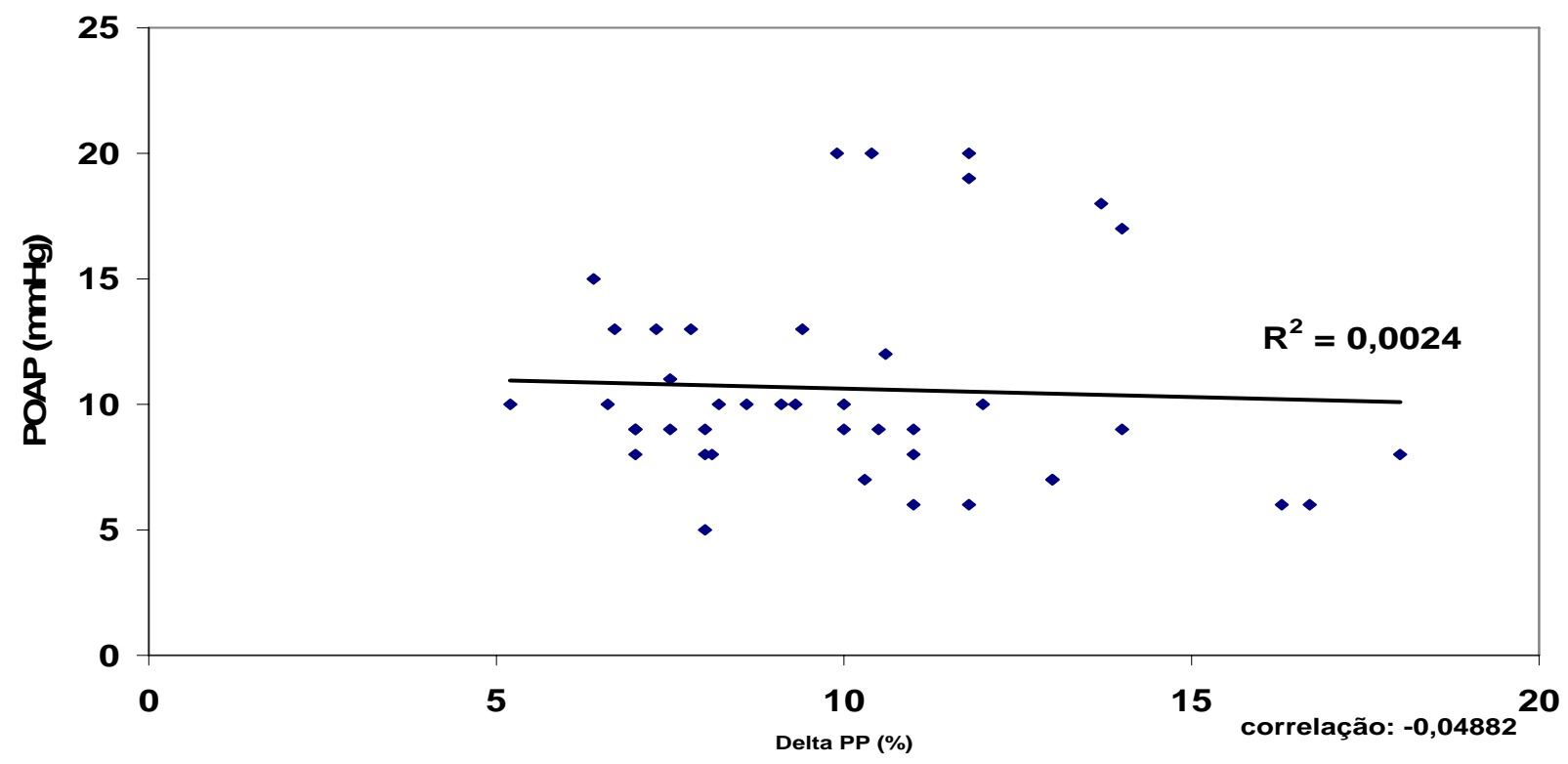

Figura 23 - Diagrama de dispersão da variação do Delta PP e POAP em porcos submetidos à ventilação controlada no grupo controle. 


\section{O HES}

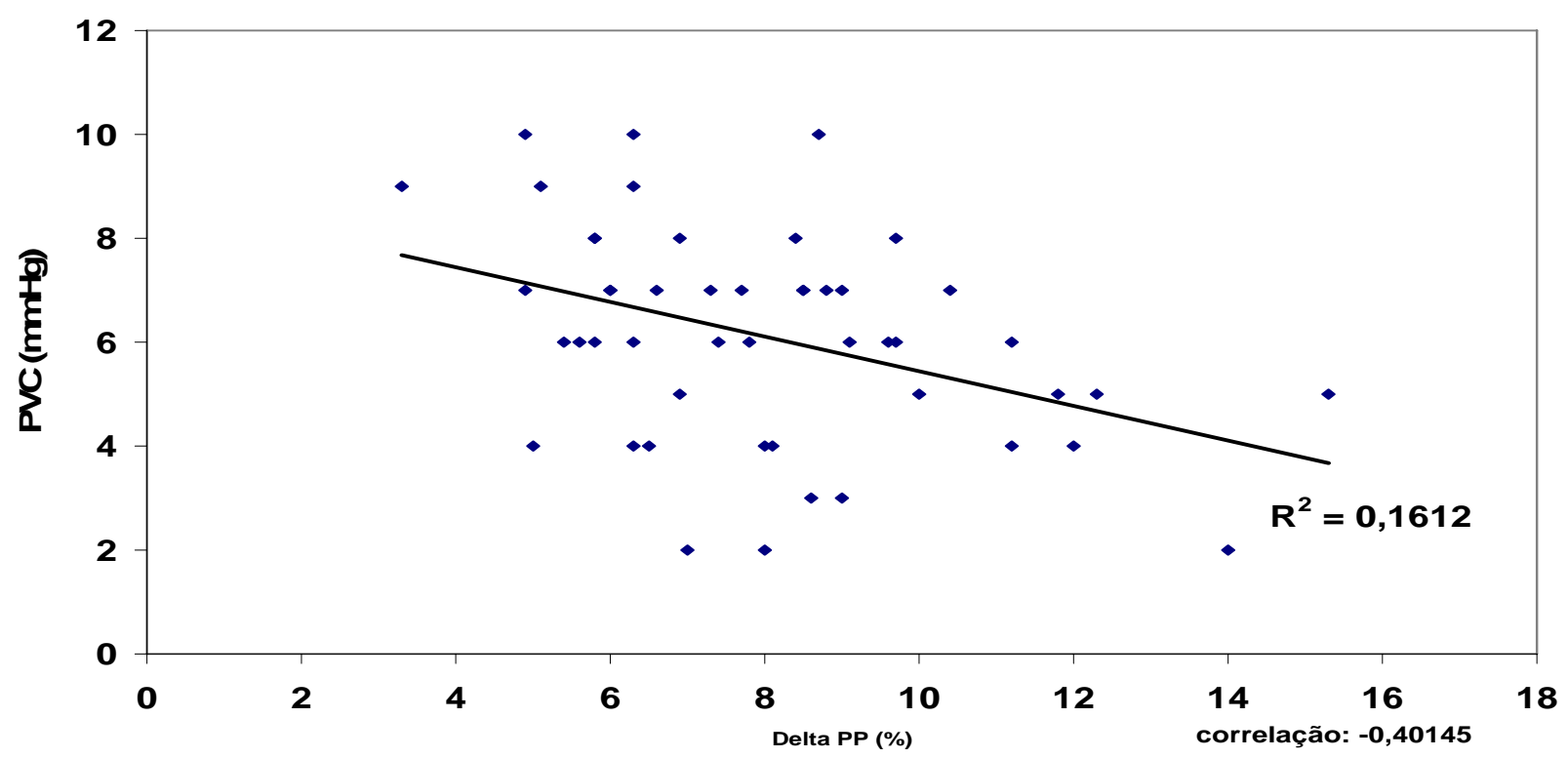

Figura 24 - Diagrama de dispersão da variação do Delta PP e PVC em porcos submetidos à ventilação controlada e hemodiluídos com amido hidroxietílico.

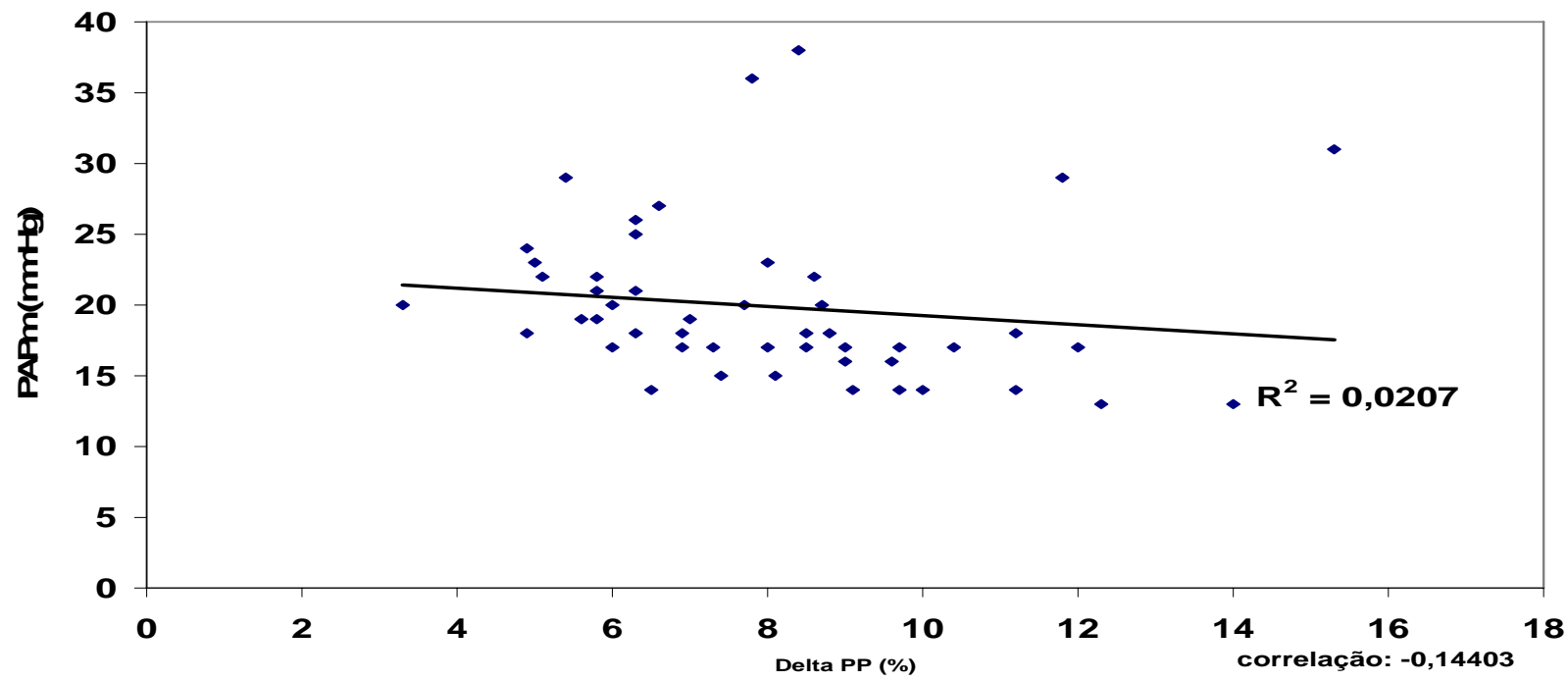

Figura 25 - Diagrama de dispersão da variação do Delta PP e PAPm em porcos submetidos à ventilação controlada e hemodiluídos com amido hidroxietílico. 


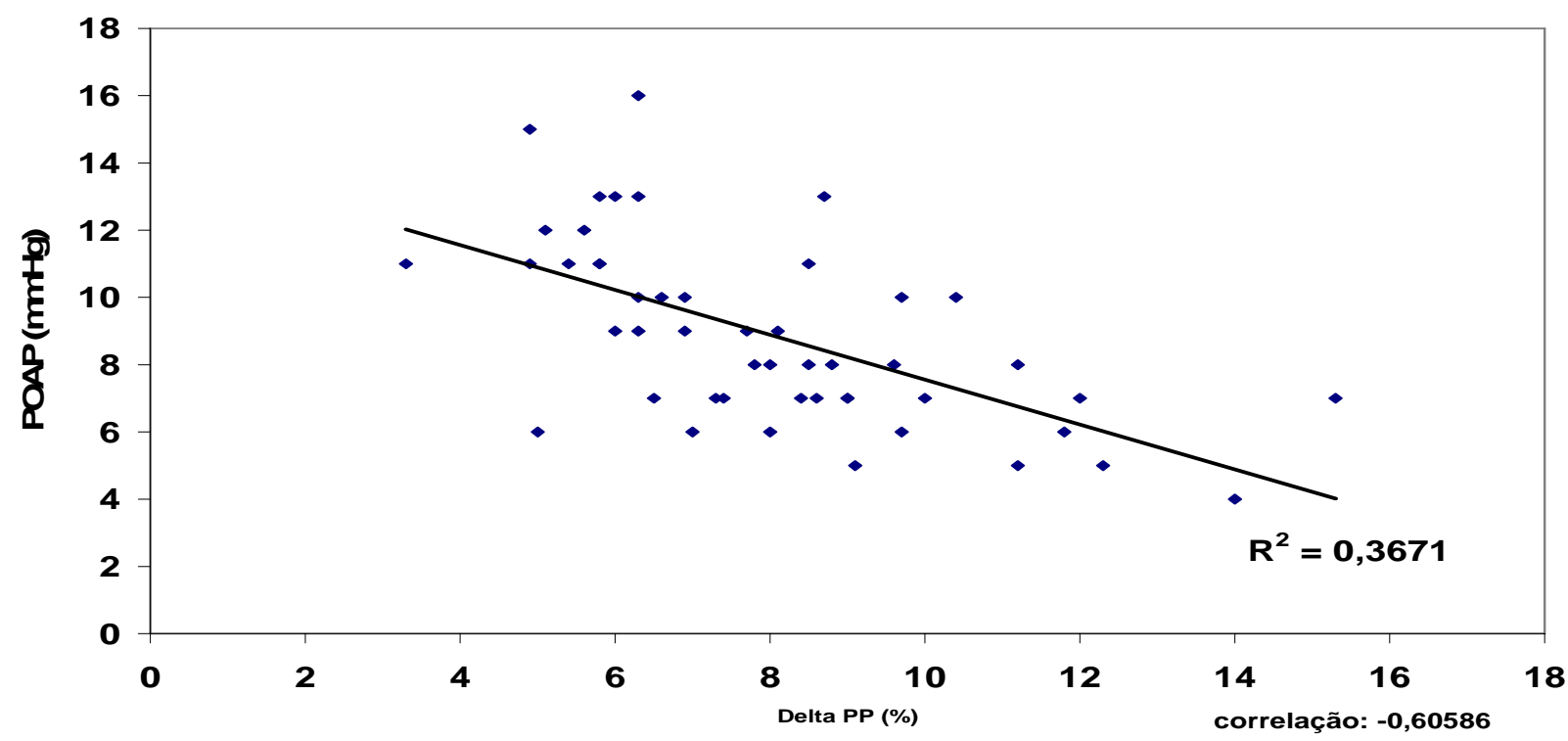

Figura 26 - Diagrama de dispersão da variação do Delta PP e POAP em porcos submetidos à ventilação controlada e hemodiluídos com amido hidroxietílico. 
o Solução salina a $0,9 \%$

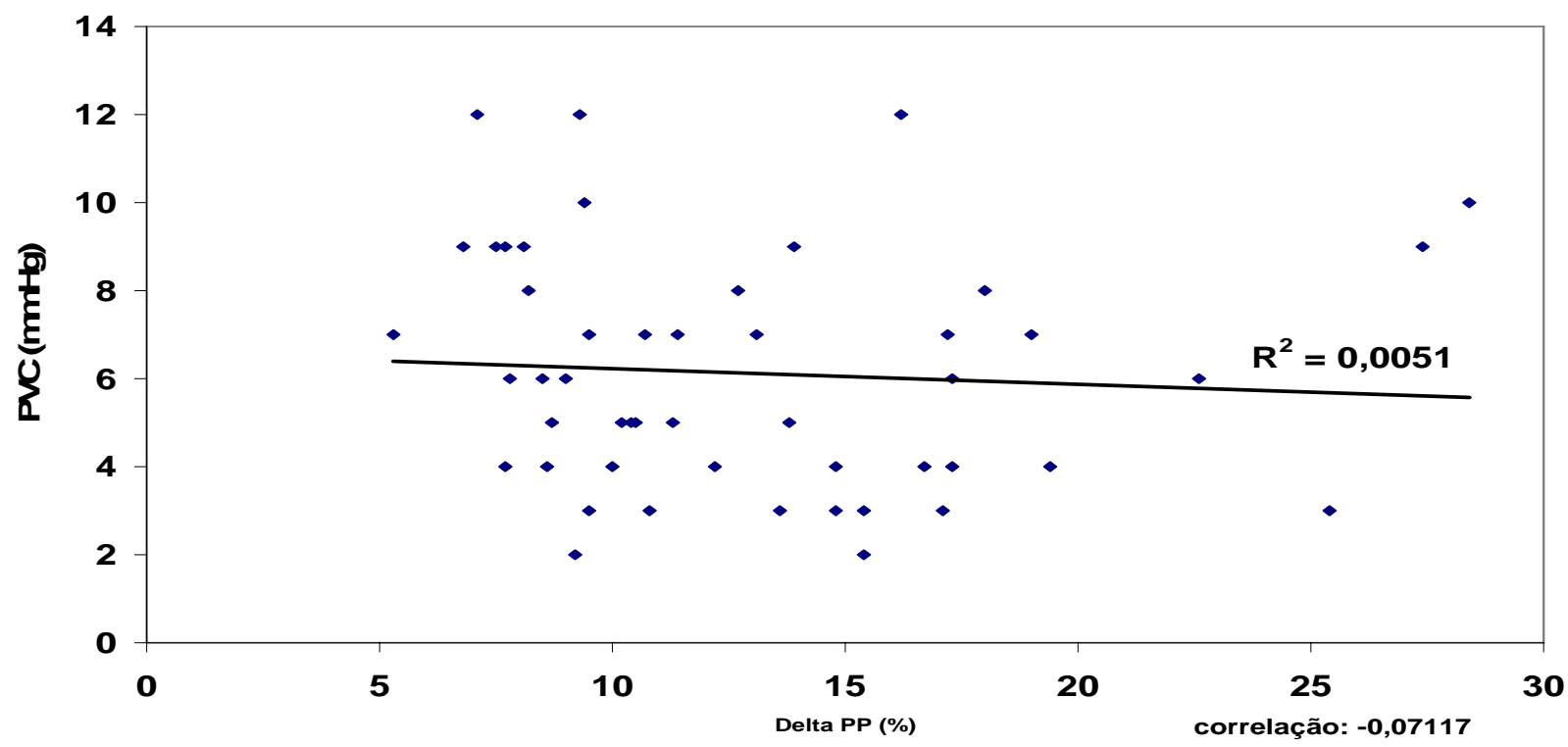

Figura 27 - Diagrama de dispersão da variação do Delta PP e PVC em porcos submetidos à ventilação controlada e hemodiluídos com Solução salina $0,9 \%$.

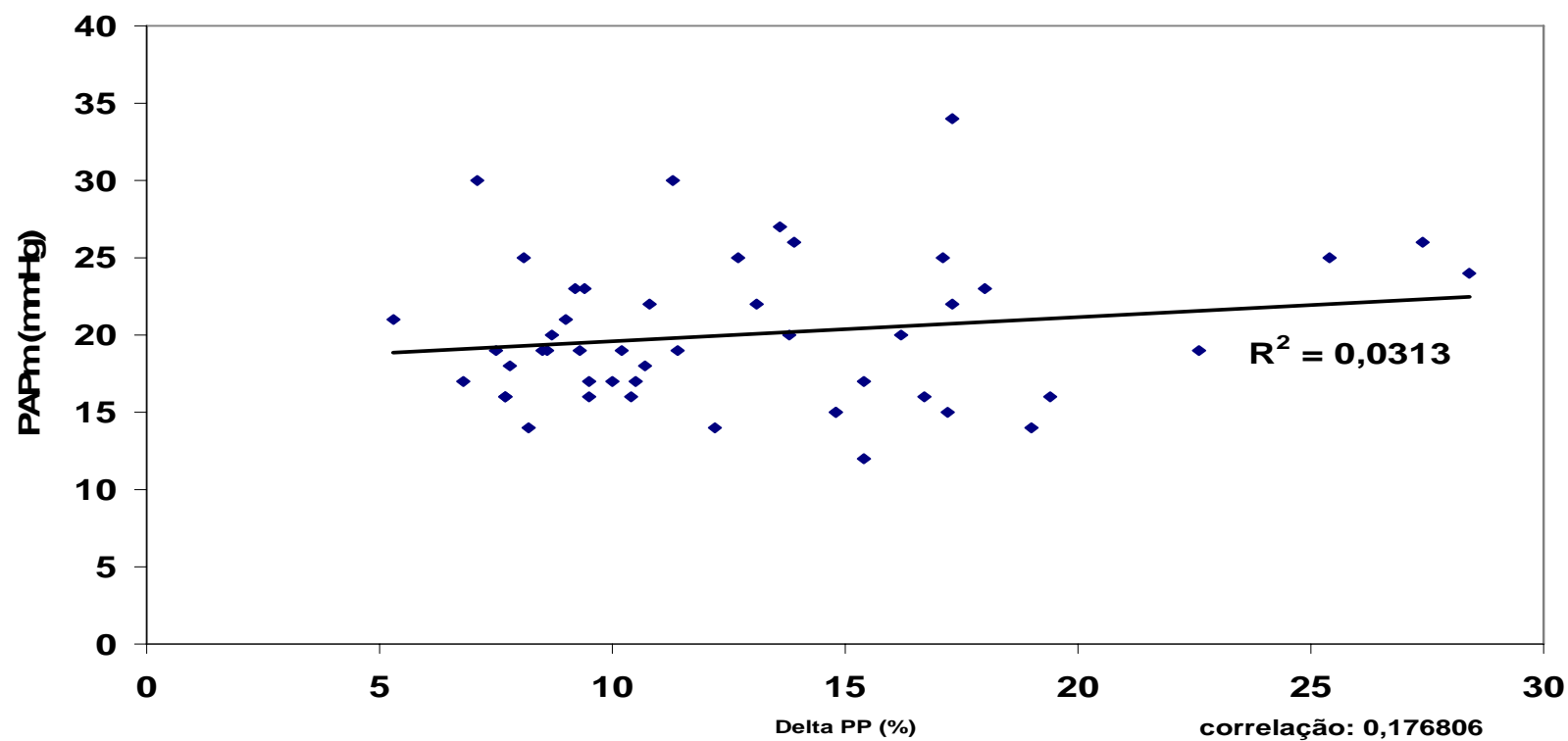

Figura 28 - Diagrama de dispersão da variação do Delta PP e PAPm em porcos submetidos à ventilação controlada e hemodiluídos com Solução salina $0,9 \%$. 


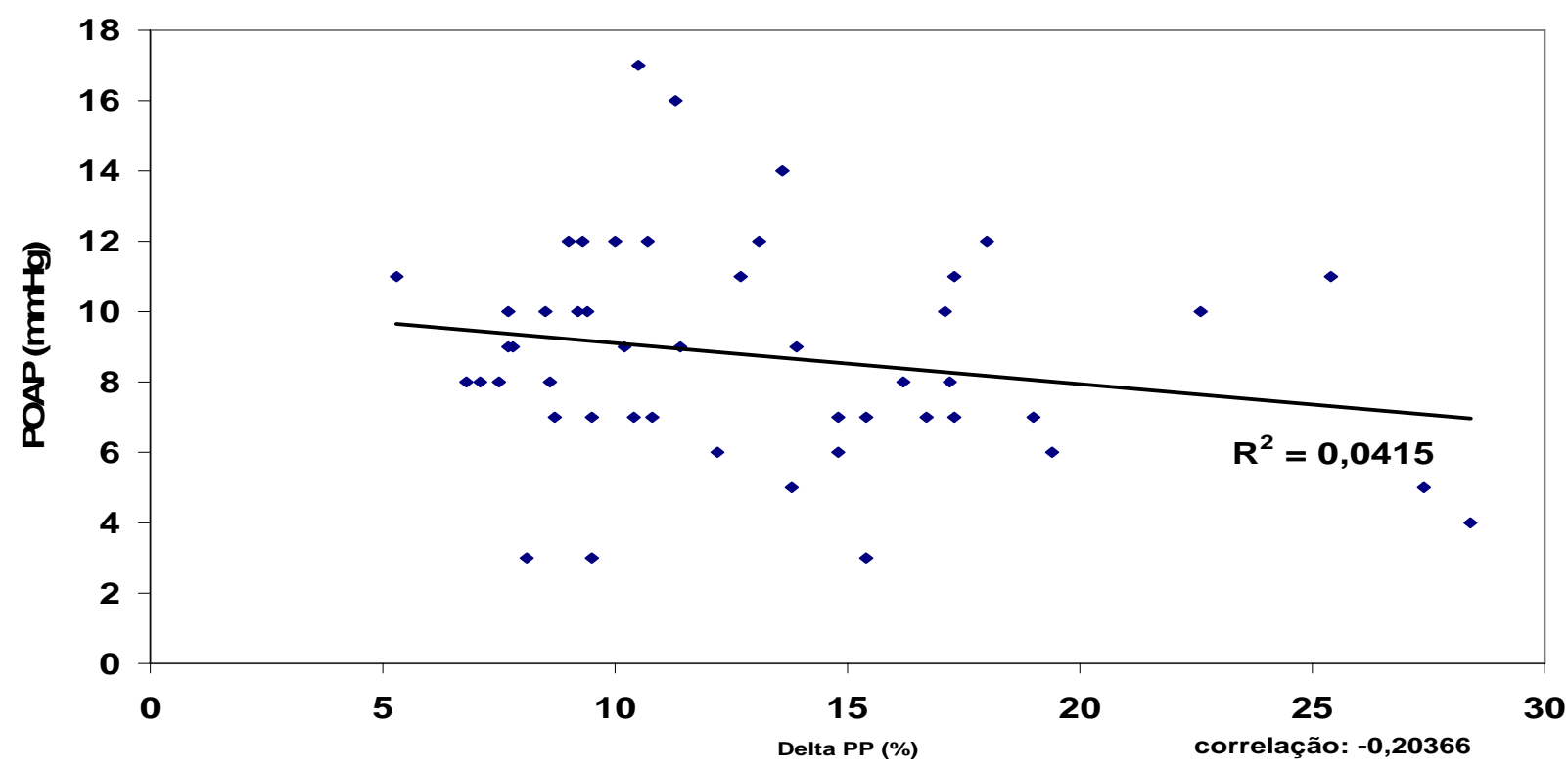

Figura 29 - Diagrama de dispersão da variação do Delta PP e POAP em porcos submetidos à ventilação controlada e hemodiluídos com Solução salina $0,9 \%$. 
6. DISCUSSÃO - 


\section{DISCUSSÃO}

O principal resultado deste estudo foi a diferença de comportamento do $\triangle \mathrm{PP}$ na hemodiluição normovolêmica aguda com dois tipos de solução de reposição, amido e solução salina. O grupo tratado com solução salina, do final do procedimento de hemodiluição até o início da retransfusão sanguínea apresentou-se com valores elevados de $\triangle \mathrm{PP}$. Enquanto o grupo tratado com amido apresentou valores de $\triangle \mathrm{PP}$ próximos ao do grupo controle. A mesma tendência também foi observada quanto à variável VPS e VVS.

A solução colóide permanece na circulação por mais tempo, o que pode explicar valores menores de $\Delta \mathrm{PP}$. Quanto aos cristalóides representados pela solução salina, o $\triangle \mathrm{PP}$ permaneceu elevado, traduzindo a fuga do fluído para o espaço extravascular. $O \Delta P P$ pode não ser uma ferramenta precisa de avaliação à responsividade volêmica quando em situações de diminuição aguda de hematócrito e reposição com soluções cristalóides.

$\mathrm{Na}$ literatura atual verifica-se uma lacuna de informações no que diz respeito à avaliação da resposta aos fluídos nos pacientes em situação de baixa hemoglobina, em anemia controlada ou não.

O modelo suíno tem sido alvo de pesquisas em hemodinâmica pela semelhança ao sistema cardiovascular humano ${ }^{32}$. 


\section{HES $(130 / 0,4)$}

O fluido ideal para reposição durante a técnica de HNA ou mesmo em pacientes graves como repositor volêmico ainda é fator de discussão na literatura $^{33}$. O amido hidroxietílico (HES 130/0,4) Voluven ${ }^{\circledR}$ - Fresenius, foi o colóide escolhido para a comparação na reposição da HNA com a solução fisiológica $0,9 \%$ pois sua efetividade tem sido tema de discussão ${ }^{9,10}$.

O objetivo principal para o uso de uma solução coloidal para reposição do volume plasmático é restabelecer a pressão oncótica sanguínea, a qual é primordialmente estabelecida pela albumina plasmática. Ao perder-se certo volume de sangue, é necessário restabelecer a pressão oncótica por meio de substitutos funcionais da albumina. O Voluven ${ }^{\circledR}$ compreende a terceira geração deste grupo de colóides, foi desenvolvida com o objetivo de manter as mesmas propriedades terapêuticas dos amidos de médio peso molecular, amplamente utilizados, como restabelecimento da pressão oncótica e expansão plasmática.

O peso molecular médio in vivo do Voluven ${ }^{\circledR}$, após sua infusão é de 70.000 - 80.000 Daltons, o qual permanece acima do limiar de filtração renal durante todo período terapêutico.

O volume de distribuição é de aproximadamente 5,9 litros. Após 30 minutos da infusão, o nível plasmático de Voluven ${ }^{\circledR}$ ainda é de $75 \%$ da concentração máxima. Após 6 horas o nível plasmático decresce 14\%. Após 24 horas de uma dose única de $500 \mathrm{ml}$ de hidroxietilamido 130/0,4 tem-se o retorno aos níveis plasmáticos iniciais. 


\section{REPOSIÇÃO VOLÊMICA E AVALIAÇÃO HEMODINÂMICA}

Atualmente, alguns estudos têm mostrado que o $\Delta \mathrm{PP}$ é um preditor da responsividade à terapia de fluidos, semelhante às variações de volume sistólico pelo contorno de pulso ou variação no período de pré-carga ${ }^{36}$. Por outro lado o $\triangle \mathrm{PP}$ tem mostrado não ser somente o mais simples, mas também o de grande especificidade em detectar a responsividade dos fluídos. Em recente estudo, Auler e colaboradores (2008) mostraram que o cálculo automático de monitorização do $\Delta \mathrm{PP}$ é possível com o uso de monitores à beira de leito e pode ser usado como preditor hemodinâmico dos efeitos da administração de fluidos intravascular em pacientes em pós operatório de cirurgia cardíaca ${ }^{37}$.

O objetivo neste estudo foi reduzir a taxa de hemoglobina promovendo um cenário de anemia normovolêmica controlada para avaliar desta forma os parâmetros dinâmicos cotejados aos estáticos frente a duas soluções de reposição volêmica. Pode-se deduzir que anemia aguda pode acontecer frequentemente durante emergência ou mesmo em cirurgias eletivas. Quantidades variadas de soluções cristalóides, colóides ou ambos podem ser usados para manter a volemia por diferentes períodos de tempo antes da transfusão sanguínea, levando a quadros de anemia aguda. Recomendações para transfusão alogênica dizem que o uso de sangue para cirurgias eletivas não deveriam ser consideradas antes de níveis de hemoglobina ao redor de 7.0 ou $6{\mathrm{~g} . \mathrm{dL}^{-1}}^{38,39}$. Esta idéia pressupõe que 
concentrações de hemoglobina durante a hemodiluição normovolêmica aguda efetiva deveriam ser ainda menores ${ }^{40}$.

$\mathrm{Na}$ hemodiluição, realizada de forma correta, a volemia é rigorosamente mantida, impedindo o hipofluxo tecidual: isquemia é evento inaceitável, implicando reinfusão imediata de sangue. Sendo assim, os estudos comparativos entre a reposição com cristalóide e colóide na ressuscitação volêmica podem apenas nortear inicialmente os estudos de hemodiluição, entretanto os resultados não são intercambiáveis.

No presente estudo a quantidade de sangue retirada na hemodiluição foi calculada, assim como a quantidade de fluido de reposição, seja colóide ou cristalóide nas devidas proporções.

No momento basal (T0) todos os dados nos três grupos apresentavam-se sem diferença significativa, o que garante a padronização dos animais de todos os grupos no início do experimento. O aumento da frequência cardíaca pode representar resposta compensatória da estimulação simpática, mas também pode ser visto como um sinal de hipovolemia $^{41}$. Nesse caso, se esperaria aumento maior no grupo hemodiluído com solução salina, que teoricamente apresenta menor efetividade na manutenção da normovolemia. Já que a resposta foi semelhante entre colóide e cristalóide, supõe-se que o aumento da frequência cardíaca resulta principalmente por resposta simpática.

O índice cardíaco apresentou um aumento compensatório esperado durante a hemodiluição ${ }^{42}$. Esse aumento ocorreu nos dois grupos tratados, no entanto, o grupo hemodiluído com solução salina a resposta traduzida em 
índice cardíaco foi menor que o daqueles tratados com HES nos momentos que compreendem o final da hemodiluição e o início da retransfusão (T2, T3, T4). Isso se deve, provavelmente, pela maior permanência do colóide no espaço intravascular. Durante a hemodiluição, além do aumento do índice cardíaco, também houve aumento do volume sistólico, frequência cardíaca e pressões de enchimento em resposta à diminuição da viscosidade do sangue. Essas respostas são determinantes da oferta de oxigênio, mesmo com a diminuição da hemoglobina ${ }^{42}$.

O aumento da taxa de extração nos dois grupos submetidos à hemodiluição é outro mecanismo compensatório à diminuição da oferta de oxigênio pela diminuição progressiva da hemoglobina e ocorre mesmo com o aumento do débito cardíaco. Quando o aumento da taxa de extração alcançar seu limite, encontra-se o ponto crítico, onde o consumo se torna dependente da oferta de oxigênio ${ }^{43}$. No presente estudo, embora ocorra aumento na taxa de extração de oxigênio ao redor de $40 \%$, não houve queda significativa no consumo de oxigênio nos grupos hemodiluídos.

Observou-se aumento do volume sistólico nos dois grupos, mas no grupo onde a reposição volêmica foi realizada com solução salina houve uma queda voltando a valores basais uma hora após o fim da hemodiluição (T3). Essa tendência em diminuição pode ser explicada pelo tempo de permanência intravascular do cristalóide ser menor que o do colóide.

A monitorização da reposição de fluidos é aspecto fundamental para que sejam evitadas as complicações causadas pela sobrecarga ou falta de volume no espaço intravascular ${ }^{44}$. Parâmetros como POAP e PVC são 
utilizados respectivamente, por $93 \%$ e $58 \%$ dos médicos, no processo de decisão para expansão volêmica ${ }^{45}$. Entretanto, estudos mostram que esses valores apresentam alguma imprecisão quanto à avaliação da volemia, em particular em pacientes sob ventilação mecânica ${ }^{46,47}$. Isto ocorre porque as mudanças no volume sistólico induzidas pela pré-carga dependem também da contratilidade e da pós-carga, que não são avaliadas por estes parâmetros ${ }^{48}$. Assim, devido às limitações dos indicadores já comentados, pautados principalmente na pressão diastólica final das câmaras cardíacas, tem surgido na literatura discussão sobre outros métodos que possam avaliar melhor a pré-carga ideal para a função cardíaca ${ }^{17,48}$.

No presente estudo observa-se aumento na PVC nos dois grupos hemodiluídos, mas apesar de apresentar diferença estatística do grupo hemodiluído com solução salina em relação ao grupo controle, verificou-se que este aumento é de apenas $1 \mathrm{mmHg}$ em média, sendo não significativo em relação a quantidade de solução infundida. Após este período de hemodiluição até a retransfusão de $50 \%$ do volume sanguíneo retirado, verificou-se uma tendência das pressões de enchimento (parâmetros estáticos) em manterem-se em valores mais baixos, principalmente no grupo hemodiluído com solução salina, onde os valores de POAP, PVC, pressão diastólica, PAM, já começam a decair antes mesmo do final da hemodiluição. Cotejando os parâmetros estáticos já citados com parâmetros dinâmicos como o $\triangle P P$, observa-se que há uma divergência de comportamento entre os grupos com soluções de reposição distintas. O $\triangle \mathrm{PP}$ e a PVC no grupo hemodiluído com solução salina entre os momentos T1 e 
T2 se comportam de forma diretamente proporcionais, parâmetros esses que deveriam se comportar inversamente proporcionais. No entanto, após esse momento o $\triangle \mathrm{PP}$ comporta-se de maneira constante e progressiva condizendo a partir de então com a PVC do grupo solução salina. Não há correlação entre o $\triangle \mathrm{PP}$ e a PVC, no grupo solução salina, com coeficiente 0,07117, no grupo HES o coeficiente de correlação entre a PVC e o $\triangle P P$ é 0,4014 . Com relação à $P O A P$ e o $\triangle P P$ do grupo solução salina há comportamento inversamente proporcional esperado em todos os momentos, com um coeficiente de correlação de -0,2036. Já o grupo HES tem comportamento estável e inversamente proporcional (esperado) entre o $\triangle \mathrm{PP}$ e as pressões de enchimento POAP e PVC, no entanto o $\triangle \mathrm{PP}$, ao contrário do grupo solução salina, no grupo HES se comporta entre valores de normovolemia, entre 10 e $15 \%^{21}$. O coeficiente de correlação do $\triangle P P$ com a PVC é $-0,4014$ e -0,6058 com a POAP no grupo HES. A VPS tem comportamento muito semelhante ao $\triangle \mathrm{PP}$ nos dois grupos hemodiluídos.

$\mathrm{O} \triangle \mathrm{PP}$, como um instrumento para detectar pacientes responsivos e não responsivos à reposição volêmica, tem sido testado e provado ser mais preciso, e extremamente sensível e específico quando substitui parâmetros hemodinâmicos tradicionais ${ }^{14,}{ }^{37}$. Entretanto indicadores dinâmicos dependem da variação respiratória e do volume de enchimento, que em pacientes, sob ventilação mecânica, tem-se mostrado superior que indicadores estáticos em predizer a responsividade à terapia de fluídos ${ }^{3,17}$.

Do ponto de vista fisiológico, a base para aplicação do $\triangle \mathrm{PP}$ na otimização da fluidoterapia consiste em maximizar o volume de enchimento 
cardíaco (até que o volume de enchimento chegue ao platô da curva de Frank-Starling). Esta estratégia requer a mensuração do volume de enchimento pela monitorização do débito cardíaco. Com o aumento da précarga cardíaca, o volume de enchimento induz a uma movimentação da relação do volume de enchimento e pré-carga e desde já uma diminuição do $\triangle P P$. Corações que se encontram no platô da curva de Frank-Starling podem ser identificados quando $\triangle \mathrm{PP}$ é baixo. Provavelmente, devido à composição molecular que mantém a pressão oncótica plasmática, amidos permanecem no espaço intravascular, o que eleva ao platô da curva de Frank- Starling e a condição volêmica do paciente ${ }^{49}$. Este fato é corroborado pelos altos valores de débito cardíaco e valores dentro da normovolemia (abaixo $12 \%$ ) de $\triangle \mathrm{PP}$ em animais tratados com amidos. Por outro lado, a infusão de cristalóides rapidamente deixa o espaço intravascular o que explica os baixos valores de débito cardíaco observados neste grupo de animais. De forma simplificada, animais hemodiluídos de forma uniforme na proporção de 3:1 de solução salina infundida e sangue removido, permanecendo, teoricamente, com a curva de Frank-Starling desviada para a esquerda, $\triangle \mathrm{PP}$ permanece com valores altos (levando em consideração o valor de $12 \%$ como limite), indicando que mais fluído deveria ser empregado. A questão principal é: deveria o $\triangle \mathrm{PP}$, em animais tratados com solução fisiológica, ter um limite estabelecido de 12\% como normal? A relação aqui pré-estabelecida e já estudada de reposição de três partes de cristalóide para cada parte de sangue removida é realmente efetiva para manutenção do estado volêmico? 
Alguns dados sobre reologia sanguínea devem ser discutidos quando se quer confrontar diferentes composições de fluídos como expansores volêmicos. O fluído melhor e mais adequado para reposição em situações de perda sanguínea, tem sido alvo de discussão, principalmente quanto as propriedades moleculares. Uma questão chave nessa controvérsia são as particularidades naturais da reologia dos expansores plasmáticos que garantem a perfusão orgânica vital. A perfusão sanguínea é determinada pela pressão de perfusão, resistência vascular, e viscosidade plasmática. Em um tubo rígido, mudanças na viscosidade dos fluídos levam a uma mudança na resistência e mútua mudança no fluxo de acordo com a equação de Poiseuille's ${ }^{50}$. Um estudo do grupo de Intaglietta utilizando hamster em modelo de microscopia intravital, o qual é muito utilizado para estudo microvascular, pôde ajudar a entender algumas questões cruciais, como, as propriedades reológicas de dois fluídos em baixas condições de hematócrito ${ }^{51}$. Utilizando esta metodologia, alguns estudos têm mostrado que se a viscosidade sanguínea é fortemente diminuída pela hemodiluição, a função microvascular é prejudicada, ocasionando uma má perfusão e distribuição do fluxo sanguíneo afetando o transporte de oxigênio, sendo perigoso para a manutenção tecidual. Claramente, um limite é alcançado quando a hemodiluição extrema não é longa, conseguindo manter os requerimentos metabólicos teciduais. Entretanto, estudos microvasculares em hemodiluição profunda mostraram que este limite pode ser significantemente menor que a "corrente de gatilhos transfusionais" se a viscosidade sanguínea é parcialmente mantida durante hemodiluição pelo 
aumento da viscosidade do plasma ${ }^{52,53}$. O efeito do aumento da viscosidade sanguínea durante hemodiluição extrema na perfusão capilar e oxigenação tecidual foi investigado por Tsai e colaboradores em modelo de microscopia intravital em hamster, com duas técnicas de hemodiluição normovolêmica, com alta ou baixa viscosidade empregando Dextran 70 a 6\%. Nesse estudo, Tsai e colaboradores mostraram que há uma diminuição de fluxo em arteríolas e vênulas depois de baixa viscosidade e somente em arteríolas depois de alta viscosidade. Foi concluído que o aumento na pressão arterial média e a diminuição da tensão são dependentes de alguns fatores de relaxamento do endotélio, e são possivelmente mecanismos que melhoram o fluxo sanguíneo em arteríolas e vênulas assim como densidade capilar depois de alta viscosidade versus baixa viscosidade ${ }^{52}$.

No presente estudo, a pressão arterial média do grupo tratado com solução salina permaneceu inferior que daqueles tratados com solução de amido, e foram parcialmente normalizados depois da retransfusão onde um esperado aumento na viscosidade deve acontecer. $\mathrm{A}$ variação do $\Delta \mathrm{PP}$ sob ventilação controlada é dependente da pré-carga e a diminuição da tensão do endotélio, o qual, na diminuição da viscosidade, pode diminuir a resistência arterial promovendo hipotensão. Então a questão chave deste estudo o qual pode explicar o comportamento distinto do $\triangle \mathrm{PP}$ sob reposição volêmica com dois tipos de fluídos, pode ser a viscosidade e peso molecular. A alteração da reologia, fluxo de fluído do espaço intravascular, ou ambos podem explicar essas diferenças no $\triangle \mathrm{PP}$ ? Uma combinação de modelos de microscopia intravital e monitorização do $\triangle \mathrm{PP}$ sob diferentes condições de 
reologia sanguínea poderiam esclarecer estas questões, mas tais procedimentos são mais distantes dos objetivos deste estudo. É importante comentar que os animais tratados com solução salina apresentaram diminuição significante nos níveis de bicarbonato de sódio, $\mathrm{pH}$ e excesso de base, as quais poderiam ser traduzidas em acidose metabólica, mas com valores normais e sem variação no lactato sérico e $\mathrm{SvO}_{2}$. Estes fatos podem ser explicados pela hipercloremia inerente ao alto volume de solução salina.

A mensuração pela ecocardiografia mostra fração de ejeção sem diferença significativa entre os dois grupos hemodiluídos à partir do início do processo, mas percentuais maiores em relação o grupo controle $(P<0,05)$. $O$ volume diastólico final não sofre alterações até a metade da hemodiluição (T1), em T2 (fim da hemodiluição) verifica-se aumento do volume diastólico final no grupo HES e diminuição no grupo solução fisiológica em relação ao controle. O grupo HES volta a valores basais e mantêm-se igual ao controle até T4 (início da retransfusão) em T5 (após retransfusão) os valores voltam a subir, mas se equivalem ao término do experimento (T6) entre os três grupos. No grupo solução salina, a partir do final da hemodiluição os valores mantêm-se baixos até o fim da retransfusão, onde elevam-se em relação ao grupo controle voltando a valores próximos do basal em T6.

Atualmente, a literatura tem mostrado a influência da ventilação mecânica no volume sistólico, que pode ser expressa na curva de pressão arterial como uma alternativa na avaliação do estado volêmico ${ }^{19,}{ }^{54-57}$. De acordo com a literatura, a magnitude das mudanças no volume sistólico 
durante a ventilação mecânica pode ser um bom indicador da dependência do débito cardíaco aos valores da pré-carga ${ }^{45}$.

Margarido e colaboradores (2007) observaram uma significante diminuição na osmolaridade sérica em animais hemodiluídos com cristalóide podendo ser esta uma causa a contribuir para o edema pulmonar. No entanto, apesar de no presente estudo terem sido utilizadas soluções cristalóides como fluido de reposição e supondo, assim, como no estudo citado de também haver promovido uma diminuição na osmolaridade, não se observou aumento da água extravascular pulmonar nos grupos hemodiluídos em relação ao grupo controle. A importância da pressão colóido-osmótica se dá por ser um mecanismo de proteção ao edema, devendo ser também considerado ${ }^{10}$.

Embora existam dificuldades para extrapolar os resultados experimentais para a prática clínica, a mensagem do estudo é centrada em que o $\triangle \mathrm{PP}$ parece não ser um indicador preciso de pré-carga quando a reposição volêmica é feita de forma maciça com cristalóides, na vigência de perda sanguínea.

Concluindo, foi possível demonstrar diferenças do $\triangle \mathrm{PP}$ como indicador de responsividade quando fluídos de diferentes viscosidades são usados para reposição volêmica. Para extrapolar esses resultados para uso clínico, mais estudos são necessários para determinar se $\Delta \mathrm{PP}$ pode ser usado em todos pacientes e, mais importante, se decisões terapêuticas baseadas nesse parâmetro pode ter alguma influência no tratamento. 


\section{CONCLUSÕES}

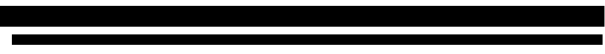




\section{CONCLUSÕES}

Com os resultados da presente pesquisa, pode-se concluir que:

A hemodiluição normovolêmica aguda reposta com amido hidroxietílico 6\% (130/04) produz maior estabilidade volêmica, quando comparada à realizada com solução cristalóide provavelmente pela meia-vida do amido no espaço intravascular ser maior que a da solução cristalóide.

$>$ Os índices dinâmicos, VPS e $\triangle \mathrm{PP}$, de responsividade à reposição volêmica possuem comportamento distinto, com diferentes fluídos de reposição, na hemodiluição normovolêmica aguda.

$\mathrm{O}$ parâmetro estático que apresentou melhor correlação com o $\Delta \mathrm{PP}$ na hemodiluição normovolêmica aguda, realizada com amido hidroxietílico, foi a pressão de oclusão de artéria pulmonar.

As mensurações de débito cardíaco por diferentes métodos (termodiluição convencional e transpulmonar) não apresenta diferença na hemodiluição normovolêmica aguda com diferentes fluídos de reposição.

As variáveis de volume de enchimento cardíaco mensuradas pela ecocardiografia aproximam-se da normalidade quando a hemodiluição 
normovolêmica aguda foi realizada com solução de amido hidroxietílico.

O índice de água extravascular pulmonar não apresentou diferença entre os grupos 
8. ANEXOS

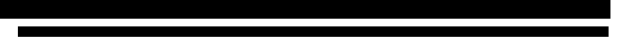


8. ANEXOS

Grupo Controle

\begin{tabular}{|c|c|c|c|}
\hline & $\begin{array}{ll}\text { VOL.INF. S.RET. } & \text { S. } \\
\end{array}$ & ASC & $\mathbf{K g}$ \\
\hline & & 0,88 & 30 \\
\hline & & 0,83 & 28 \\
\hline & & 0,94 & 34 \\
\hline & & 0,91 & 32,4 \\
\hline & & 0,92 & 33 \\
\hline & & 0,81 & 27,5 \\
\hline & & 0,96 & 35 \\
\hline Média & & 0,89 & 31,41 \\
\hline DP & & 0,06 & 2,94 \\
\hline
\end{tabular}

Grupo HES

\begin{tabular}{|c|c|c|c|c|}
\hline & VOL.INF. & S.RET. & ASC & Kg \\
\hline & 1371 & 1371 & 0,88 & 30,5 \\
\hline & 1317,85 & 1317,85 & 0,88 & 30,7 \\
\hline & 1264 & 1264 & 0,9 & 31,6 \\
\hline & 1538,23 & 1538,23 & 0,9 & 31,8 \\
\hline & 1600 & 1600 & 0,95 & 34,4 \\
\hline & 1499,5 & 1499,5 & 0,88 & 31 \\
\hline & 1644 & 1644 & 0,94 & 34 \\
\hline Média & 1462,1 & 1462,1 & 0,90 & 32,00 \\
\hline DP & 145,9 & 145,9 & 0,03 & 1,58 \\
\hline \multicolumn{5}{|c|}{ Grupo solução salina } \\
\hline & VOL.INF. & S.RET. & ASC & $\mathbf{K g}$ \\
\hline & 3600 & 1200 & 0,87 & 30 \\
\hline & 3600 & 1200 & 0,87 & 30 \\
\hline & 3324 & 1108 & 0,93 & 33 \\
\hline & 3861 & 1287 & 0,87 & 30 \\
\hline & 4643,7 & 1547,9 & 0,91 & 32 \\
\hline & 5514,42 & 1838,14 & 1,02 & 38 \\
\hline & 5600,01 & 1866,67 & 0,96 & 35 \\
\hline & 3321 & 1107 & 0,86 & 30 \\
\hline Média & 4183,0 & 1394,3 & 0,91 & 32,14 \\
\hline DP & 945,5 & 315,2 & 0,06 & 3,18 \\
\hline
\end{tabular}


Grupo controle DÉBITO URINÁRIO mL

\begin{tabular}{|c|c|c|c|c|c|c|c|}
\hline ANIMAL & T0 & T1 & T2 & T3 & T4 & T5 & T6 \\
\hline 1 & & 130 & 150 & 204 & 240 & 350 & 342 \\
\hline 2 & & 120 & 160 & 204 & 250 & 200 & 350 \\
\hline 3 & & 100 & 120 & 170 & 200 & 200 & 230 \\
\hline 4 & & 100 & 150 & 200 & 300 & 350 & 400 \\
\hline 5 & & 120 & 150 & 200 & 220 & 280 & 380 \\
\hline 6 & & 130 & 200 & 250 & 300 & 330 & 350 \\
\hline 7 & & 150 & 180 & 200 & 230 & 280 & 350 \\
\hline Média & & 121 & 159 & 204 & 249 & 284 & 343 \\
\hline DP & & 18 & 25 & 24 & 38 & 65 & 54 \\
\hline \multicolumn{2}{|c|}{ Grupo HES } & \multicolumn{2}{|c|}{ DÉBITO URINÁRIO } & & & & \\
\hline ANIMAL & T0 & T1 & T2 & T3 & T4 & T5 & T6 \\
\hline 1 & & 100 & 180 & 300 & 400 & 500 & 600 \\
\hline 2 & & 180 & 290 & 300 & 400 & 660 & 520 \\
\hline 4 & & 300 & 600 & 900 & 1000 & 1100 & 1200 \\
\hline 7 & & 180 & 320 & 500 & 600 & 650 & 700 \\
\hline 8 & & 60 & 100 & 35 & 500 & 550 & 70 \\
\hline 9 & & 250 & 300 & 400 & 50 & 550 & 600 \\
\hline 10 & & 200 & 250 & 500 & 550 & 630 & 800 \\
\hline Média & & 181 & 291 & 419 & 500 & 663 & 641 \\
\hline \multirow[t]{2}{*}{ DP } & & 82 & 156 & 265 & 284 & 202 & 338 \\
\hline & \multicolumn{3}{|c|}{ Grupo Solução salina } & \multicolumn{2}{|c|}{ DÉBITO URINÁRIO } & & \\
\hline ANIMAL & T0 & T1 & T2 & T3 & T4 & T5 & T6 \\
\hline 1 & & 550 & 1200 & 1700 & 2100 & 2500 & 1800 \\
\hline 2 & & 400 & 900 & 1200 & 1600 & 1800 & 1900 \\
\hline 3 & & 800 & 1400 & 2800 & 3600 & 4100 & 4600 \\
\hline 4 & & 100 & 600 & 1000 & 1100 & 1200 & 1400 \\
\hline 5 & & 500 & 1000 & 1400 & 1700 & 2000 & 2500 \\
\hline 6 & & 500 & 1100 & 2000 & 2300 & 2700 & 3000 \\
\hline 7 & & 1000 & 1900 & 2200 & 2700 & 3000 & 3300 \\
\hline Média & & 550 & 1157 & 1757 & 2157 & 2471 & 2643 \\
\hline DP & & 287 & 412 & 627 & 820 & 938 & 1097 \\
\hline
\end{tabular}




\begin{tabular}{|c|c|c|c|c|c|c|c|}
\hline \multicolumn{2}{|c|}{ Grupo controle } & \multicolumn{2}{|c|}{ Delta PP automático } & \multicolumn{4}{|l|}{$\%$} \\
\hline ANIMAL & T0 & T1 & T2 & T3 & T4 & T5 & T6 \\
\hline 1 & 12 & 9,8 & 14 & 18 & 10 & 11 & 10,5 \\
\hline 2 & 8 & 8 & 7 & 7 & 8 & 13 & 9,5 \\
\hline 3 & 11,8 & 10,3 & 13 & 11 & 16,7 & 16,3 & 14,3 \\
\hline 4 & 5,2 & 7,5 & 7 & 6,6 & 9,3 & 11 & 10 \\
\hline 5 & 12,8 & 14 & 13,7 & 10,4 & 11,8 & 11,8 & 9,9 \\
\hline 6 & 6,2 & 9,4 & 10,6 & 6,7 & 6,4 & 7,8 & 7,3 \\
\hline 7 & 7,5 & 8,1 & 9,1 & 8,6 & 8 & 8,2 & 10,5 \\
\hline Média & 9,1 & 9,6 & 10,6 & 9,8 & 10,0 & 11,3 & 10,3 \\
\hline DP & 3,1 & 2,2 & 3,0 & 4,0 & 3,4 & 2,9 & 2,1 \\
\hline \multicolumn{2}{|c|}{ Grupo HES } & \multicolumn{2}{|c|}{ Delta PP automático } & & & & \\
\hline ANIMAL & T0 & T1 & T2 & T3 & T4 & T5 & T6 \\
\hline 1 & 12 & 10 & 9,6 & 11,2 & 14 & 11,2 & 7,7 \\
\hline 2 & 9 & 8,8 & 8,5 & 12,3 & 9,7 & 6,9 & 9,1 \\
\hline 4 & 7 & 6,3 & 5,4 & 8 & 8,6 & 6,6 & 5 \\
\hline 7 & 5,8 & 6 & 3,3 & 7,8 & 15,3 & 8,4 & 11,8 \\
\hline 8 & 9,7 & 6,3 & 4,9 & 6 & 7,4 & 5,1 & 7,3 \\
\hline 9 & 8,1 & 6,9 & 4,9 & 6,5 & 9 & 5,8 & 8 \\
\hline 10 & 10,4 & 8,7 & 5,8 & 6,3 & 8,5 & 6,3 & 5,6 \\
\hline Média & 8,9 & 7,6 & 6,1 & 8,3 & 10,4 & 7,2 & 7,8 \\
\hline \multirow[t]{2}{*}{ DP } & 2,1 & 1,6 & 2,2 & 2,5 & 3,0 & 2,0 & 2,3 \\
\hline & \multicolumn{3}{|c|}{ Grupo Sol. salina } & \multicolumn{3}{|c|}{ Delta PP automático } & \\
\hline ANIMAL & T0 & T1 & T2 & T3 & T4 & T5 & T6 \\
\hline 1 & 8,2 & 7,7 & 9,3 & 17,2 & 19 & 9,4 & 6,8 \\
\hline 2 & 11,4 & 9 & 18 & 22,6 & 17,3 & 12,7 & 13,1 \\
\hline 3 & 7,8 & 10,2 & 12,2 & 13,8 & 15,4 & 8,7 & 9,5 \\
\hline 4 & 10 & 10,5 & 10,7 & 14,8 & 15,4 & 5,3 & 7,7 \\
\hline 5 & 7,5 & 13,9 & 16,2 & 27,4 & 28,4 & 7,1 & 8,1 \\
\hline 6 & 10,8 & 9,2 & 17,3 & 25,4 & 17,1 & 11,3 & 13,6 \\
\hline 7 & 9,5 & 10,4 & 14,8 & 16,7 & 19,4 & 8,5 & 8,6 \\
\hline Média & 9,3 & 10,1 & 14,1 & 19,7 & 18,9 & 9,0 & 9,6 \\
\hline DP & 1,5 & 1,9 & 3,4 & 5,4 & 4,5 & 2,5 & 2,7 \\
\hline
\end{tabular}




\begin{tabular}{cccccccc}
\multicolumn{2}{c}{ Grupo controle } & \multicolumn{2}{l}{ VPS automático } & $\mathbf{m m H g}$ & & & \\
\hline ANIMAL & T0 & T1 & T2 & T3 & T4 & T5 & T6 \\
\hline $\mathbf{1}$ & 7 & & 7 & 8 & 6 & 7 & \\
$\mathbf{2}$ & 3 & 3 & 3 & 3 & 3 & 4 & \\
$\mathbf{3}$ & 7,5 & 5,6 & 6,3 & 8,1 & 10 & 9,2 & 9,1 \\
$\mathbf{4}$ & 2,3 & 3 & 3,1 & 2,5 & 4,5 & 4,5 & 5 \\
$\mathbf{5}$ & 6,5 & 7,3 & 7,1 & 6,1 & 6,8 & 6,8 & 6 \\
$\mathbf{6}$ & 3,4 & 4,9 & 5,3 & 4,4 & 4,7 & 5,7 & 6,3 \\
$\mathbf{7}$ & 4,7 & 5,3 & 5,6 & 6,1 & 6,3 & 5,6 & 6,3 \\
\hline Média & 4,9 & 4,9 & 5,3 & 5,5 & 5,9 & 6,1 & 6,5 \\
DP & 2,1 & 1,7 & 1,7 & 2,2 & 2,2 & 1,7 & 1,5 \\
\hline & & & & & & & \\
Grupo HES & VPS automático & & & & \\
\hline ANIMAL & T0 & T1 & T2 & T3 & T4 & T5 & T6 \\
\hline $\mathbf{1}$ & 9,4 & 8,1 & 6,8 & 10,1 & 11,8 & 7,3 & 8,2 \\
$\mathbf{2}$ & 5,5 & 4,9 & 4,9 & 6,9 & 6,9 & 3,3 & 5,5 \\
$\mathbf{4}$ & 4,9 & 4,4 & 3,9 & 6,5 & 6,5 & 4,5 & 4,7 \\
$\mathbf{7}$ & 3,8 & 3,6 & 3,8 & 6,6 & 10,8 & 5,4 & 8,5 \\
$\mathbf{8}$ & 5,8 & 3,5 & 3 & 4,2 & 5,3 & 2,6 & 3,5 \\
$\mathbf{9}$ & 3,2 & 3,5 & 3,1 & 3,5 & 4,4 & 3,9 & 3,7 \\
$\mathbf{1 0}$ & 4,6 & 3,7 & 3,3 & 4,5 & 4,4 & 3,1 & 3,2 \\
\hline Média & 5,3 & 4,5 & 4,1 & 6,0 & 7,2 & 4,3 & 5,3 \\
DP & 2,0 & 1,7 & 1,3 & 2,2 & 3,0 & 1,6 & 2,2 \\
\hline
\end{tabular}

Grupo Sol. salina

\begin{tabular}{cccccccc} 
& \multicolumn{7}{c}{ VPS automático } \\
\hline ANIMAL & T0 & T1 & T2 & T3 & T4 & T5 & T6 \\
\hline $\mathbf{1}$ & $\mathbf{5 , 1}$ & $\mathbf{6 , 6}$ & $\mathbf{6 , 2}$ & $\mathbf{9 , 9}$ & $\mathbf{1 0 , 8}$ & $\mathbf{5 , 8}$ & $\mathbf{5}$ \\
$\mathbf{2}$ & 6,2 & 4,7 & 8 & 10,6 & 7 & 4,9 & 5,7 \\
$\mathbf{3}$ & 5,5 & 6,2 & 6,7 & 8 & 9 & 4,9 & 6,1 \\
$\mathbf{4}$ & 5 & 7,3 & 6,4 & 8,2 & 8,3 & 3,3 & 4,2 \\
$\mathbf{5}$ & 4,6 & 9,8 & 13 & 17,5 & 16,8 & 7,3 & 7,9 \\
$\mathbf{6}$ & 8,3 & 9,9 & 18,6 & 14,4 & 14,9 & 9,3 & 9 \\
$\mathbf{7}$ & 4,8 & 5,5 & 8,9 & 11,7 & 10,9 & 6,9 & 6,6 \\
\hline Média & 5,6 & 7,1 & 9,7 & 11,5 & 11,1 & 6,1 & 6,4 \\
DP & 1,3 & 2,0 & 4,6 & 3,4 & 3,6 & 2,0 & 1,7 \\
\hline
\end{tabular}




\begin{tabular}{cccccccc}
\multicolumn{2}{l}{ Grupo controle } & Delta up & \multicolumn{7}{c}{$\mathbf{~ m m H g}$} \\
\hline ANIMAL & T0 & T1 & T2 & T3 & T4 & T5 & T6 \\
\hline $\mathbf{1}$ & 5 & & 6 & 7 & 6 & 7 & \\
$\mathbf{2}$ & 4 & 2 & 2 & 2 & 2 & 4 & \\
$\mathbf{3}$ & 4,7 & 3,5 & 4,2 & 5 & 5,1 & 5,1 & 4,6 \\
$\mathbf{4}$ & 1,5 & 2 & 1,6 & 1,9 & 2,5 & 2,7 & 3 \\
$\mathbf{5}$ & 4 & 4,3 & 3,5 & 3,7 & 3,5 & 4,2 & 3,2 \\
$\mathbf{6}$ & 0,8 & 2,3 & 2,8 & 2,1 & 2,6 & 3 & 3,1 \\
$\mathbf{7}$ & 2,9 & 3,3 & 3,1 & 3,5 & 5,7 & 3,5 & 3,1 \\
\hline Média & 3,3 & 2,9 & 3,3 & 3,6 & 3,9 & 4,2 & 3,4 \\
DP & 1,6 & 0,9 & 1,5 & 1,9 & 1,7 & 1,5 & 0,7 \\
\hline
\end{tabular}

\section{Grupo HES Delta up}

\begin{tabular}{|c|c|c|c|c|c|c|c|}
\hline ANIMAL & T0 & T1 & T2 & T3 & T4 & T5 & T6 \\
\hline 1 & 3,9 & 4 & 1,8 & 3,1 & 3 & 4 & 3,7 \\
\hline 2 & 2,4 & 2,8 & 3,1 & 3,8 & 3,7 & 2,1 & 3,4 \\
\hline 4 & 2 & 2,3 & 2 & 2,5 & 4 & 2,1 & 1,8 \\
\hline 7 & 2,2 & 2,2 & 1,7 & 2,9 & 2,6 & 2,9 & 2,6 \\
\hline 8 & 3,6 & 2,6 & 2,1 & 3,1 & 2,9 & 2 & 2,5 \\
\hline 9 & 2,4 & 2,1 & 2,1 & 2,2 & 2,9 & 2,4 & 2,6 \\
\hline 10 & 3,4 & 2,7 & 1,8 & 2 & 2,6 & 1,6 & 1,6 \\
\hline Média & 2,8 & 2,7 & 2,1 & 2,8 & 3,1 & 2,4 & 2,6 \\
\hline DP & 0,8 & 0,6 & 0,5 & 0,6 & 0,5 & 0,8 & 0,8 \\
\hline \multicolumn{4}{|c|}{ Grupo Sol. salina } & \multicolumn{4}{|c|}{ Delta up } \\
\hline ANIMAL & TO & T1 & T2 & T3 & T4 & T5 & T6 \\
\hline 1 & 3 & 4 & 3,9 & 5,8 & 5,7 & 3,5 & $\overline{2,9}$ \\
\hline 2 & 4,1 & 2,8 & 4,1 & 4,7 & 4,2 & 2,8 & 3,4 \\
\hline 3 & 2,5 & 3,3 & 3,5 & 3,3 & 3,8 & 2,9 & 3,1 \\
\hline 4 & 1,9 & 2,1 & 2,6 & 2,4 & 2,6 & 2,1 & 2,4 \\
\hline 5 & 2,5 & 4,2 & 5,3 & 8,6 & 5 & 3,2 & 3,7 \\
\hline 6 & 2,8 & 2,3 & 2,7 & 3,3 & 2,9 & 4,1 & 4,3 \\
\hline 7 & 3 & 3,1 & 3,8 & 3,4 & 4,1 & 2,5 & 2,1 \\
\hline Média & 2,8 & 3,1 & 3,7 & 4,5 & 4,0 & 3,0 & 3,1 \\
\hline DP & 0,7 & 0,8 & 0,9 & 2,1 & 1,1 & 0,7 & 0,8 \\
\hline
\end{tabular}




\begin{tabular}{cccccccc}
\multicolumn{2}{l}{ Grupo controle } & Delta down & \multicolumn{5}{c}{$\mathbf{\text { mm Hg }}$} \\
\hline ANIMAL & T0 & T1 & T2 & T3 & T4 & T5 & T6 \\
\hline $\mathbf{1}$ & 2 & & 1 & 2 & 1 & 1 & \\
$\mathbf{2}$ & 0 & 2 & 1 & 2 & 1 & 1 & \\
$\mathbf{3}$ & 2,9 & 2,1 & 2,2 & 3,1 & 4,9 & 4,2 & 4,5 \\
$\mathbf{4}$ & 0,8 & 1 & 1,5 & 0,6 & 2,1 & 1,9 & 2 \\
$\mathbf{5}$ & 2,7 & 2,9 & 3,8 & 2,4 & 2,3 & 2,6 & 2,9 \\
$\mathbf{6}$ & 2,6 & 2,7 & 2,6 & 2,1 & 2,1 & 2,6 & 3,2 \\
$\mathbf{7}$ & 1,9 & 1,9 & 2,5 & 2,7 & 0,6 & 2,1 & 3,1 \\
\hline Média & 1,8 & 2,1 & 2,1 & 2,1 & 2,0 & 2,2 & 3,1 \\
DP & 1,1 & 0,7 & 1,0 & 0,8 & 1,4 & 1,1 & 0,9 \\
\hline
\end{tabular}

\section{Grupo HES}

\section{Delta down}

\begin{tabular}{cccccccc}
\hline ANIMAL & T0 & T1 & T2 & T3 & T4 & T5 & T6 \\
\hline $\mathbf{1}$ & 5,6 & 4,1 & 5,1 & 7 & 8,7 & 3,1 & 4,4 \\
$\mathbf{2}$ & 2,9 & 2 & 1,8 & 3,1 & 3,2 & 1,3 & 2,1 \\
$\mathbf{4}$ & 2,8 & 2,2 & 1,9 & 4,1 & 2,7 & 2,3 & 2,9 \\
$\mathbf{7}$ & 1,8 & 1,4 & 2,1 & 3,6 & 8,3 & 2,4 & 5,9 \\
$\mathbf{8}$ & 2,2 & 0,8 & 0,9 & 1,2 & 2,2 & 0,6 & 1 \\
$\mathbf{9}$ & 0,7 & 1,3 & 1,1 & 1,4 & 1,5 & 1,6 & 1,1 \\
$\mathbf{1 0}$ & 1,5 & 1,2 & 1,5 & 2,6 & 1,8 & 1,4 & 1,5 \\
\hline Média & 2,5 & 1,9 & 2,1 & 3,3 & 4,1 & 1,8 & 2,7 \\
DP & 1,6 & 1,1 & 1,4 & 2,0 & 3,1 & 0,8 & 1,8 \\
\hline
\end{tabular}

Grupo Sol. salina

Delta down

\begin{tabular}{cccccccc}
\hline ANIMAL & T0 & T1 & T2 & T3 & T4 & T5 & T6 \\
\hline $\mathbf{1}$ & $\mathbf{2}$ & $\mathbf{2 , 7}$ & $\mathbf{2 , 1}$ & $\mathbf{4 , 1}$ & $\mathbf{5 , 1}$ & $\mathbf{2 , 3}$ & $\mathbf{2 , 2}$ \\
$\mathbf{2}$ & 2,3 & 2 & 3,7 & 5,5 & 2,9 & 1,7 & 2,3 \\
$\mathbf{3}$ & 2,3 & 2,9 & 2,9 & 4,6 & 4,9 & 2 & 3 \\
$\mathbf{4}$ & 2,6 & 5,2 & 3,9 & 5,8 & 5,6 & 1,3 & 1,8 \\
$\mathbf{5}$ & 2 & 5,6 & 7,9 & 8,9 & 11,8 & 4,2 & 4 \\
$\mathbf{6}$ & 5,4 & 7,6 & 16,7 & 11,2 & 12 & 5,2 & 4,7 \\
$\mathbf{7}$ & 1,8 & 2,7 & 5,1 & 8,5 & 6,7 & 4,5 & 4,5 \\
\hline Média & 2,6 & 4,1 & 6,0 & 6,9 & 7,0 & 3,0 & 3,2 \\
DP & 1,2 & 2,1 & 5,1 & 2,6 & 3,5 & 1,6 & 1,2 \\
\hline
\end{tabular}




\begin{tabular}{cccccccc} 
& \multicolumn{2}{c}{ Grupo controle } & \multicolumn{2}{c}{ DC termodiluição } & L/min & & \\
\hline ANIMAL & T0 & T1 & T2 & T3 & T4 & T5 & T6 \\
\hline $\mathbf{1}$ & 4,2 & 4 & 3,6 & 3,8 & 3,3 & 3 & 3,2 \\
$\mathbf{2}$ & 4,5 & 4 & 4 & 4,5 & 4,3 & 4 & 4,5 \\
$\mathbf{3}$ & 4 & 3,9 & 3,5 & 3,8 & 3,5 & 3,4 & 3,5 \\
$\mathbf{4}$ & 5 & 4,4 & 4,9 & 4,8 & 5 & 4,6 & 4,7 \\
$\mathbf{5}$ & 5 & 4,4 & 4,5 & 4,4 & 4,3 & 4,8 & 4,8 \\
$\mathbf{6}$ & 4 & 3,7 & 4,1 & 3,7 & 3,6 & 3,6 & 3,6 \\
$\mathbf{7}$ & 5 & 3,9 & 4,2 & 4,6 & 5,3 & 4,7 & 4,5 \\
\hline Média & 4,5 & 4,0 & 4,1 & 4,2 & 4,2 & 4,0 & 4,1 \\
DP & 0,5 & 0,3 & 0,5 & 0,4 & 0,8 & 0,7 & 0,7 \\
\hline
\end{tabular}

Grupo HES DC termodiluição

\begin{tabular}{cccccccc}
\hline ANIMAL & T0 & T1 & T2 & T3 & T4 & T5 & T6 \\
\hline $\mathbf{1}$ & 5,1 & 6,8 & 8,4 & 6,8 & 6,4 & 5,8 & 6,5 \\
$\mathbf{2}$ & 5 & 5,8 & 6,4 & 4,8 & 5,7 & 6 & 5,1 \\
$\mathbf{3}$ & 4,9 & 6 & 7 & 5,5 & 4,8 & 5,1 & 4,6 \\
$\mathbf{4}$ & 5,8 & 7 & 8,4 & 6,8 & 6,4 & 5,8 & 6,1 \\
$\mathbf{5}$ & 5,1 & 6,8 & 8,6 & 7,1 & 6,5 & 7 & 6 \\
$\mathbf{6}$ & 4 & 6,4 & 7,6 & 5,9 & 5,8 & 6,5 & 5,2 \\
$\mathbf{7}$ & 5,1 & 6,4 & 8,4 & 7,3 & 6,8 & 7,8 & 7,1 \\
\hline Média & 5,0 & 6,5 & 7,8 & 6,3 & 6,1 & 6,3 & 5,8 \\
DP & 0,5 & 0,4 & 0,9 & 0,9 & 0,7 & 0,9 & 0,9 \\
\hline
\end{tabular}

Grupo Solução salina DC termodiluição

\begin{tabular}{cccccccc}
\hline ANIMAL & T0 & T1 & T2 & T3 & T4 & T5 & T6 \\
\hline $\mathbf{1}$ & 4,6 & 5,9 & 7 & 4,6 & 4,8 & 5,5 & 6,3 \\
$\mathbf{2}$ & 4,7 & 4,7 & 4,6 & 3,7 & 3,8 & 3,9 & 3,7 \\
$\mathbf{3}$ & 5,1 & 7 & 6,5 & 6,4 & 5,6 & 6 & 6,2 \\
$\mathbf{4}$ & 5,7 & 7,9 & 7,8 & 5,5 & 5,4 & 7 & 6,7 \\
$\mathbf{5}$ & 4,9 & 7,6 & 7,2 & 6,9 & 5,3 & 7 & 6,5 \\
$\mathbf{6}$ & 5,3 & 7,8 & 7,2 & 5,4 & 4,6 & 6,7 & 7 \\
$\mathbf{7}$ & 3,7 & 5 & 5,9 & 5,3 & 6,1 & 6,6 & 5,5 \\
\hline Média & 4,9 & 6,6 & 6,6 & 5,4 & 5,1 & 6,1 & 6,0 \\
DP & 0,6 & 1,4 & 1,1 & 1,1 & 0,8 & 1,1 & 1,1 \\
\hline
\end{tabular}




\begin{tabular}{|c|c|c|c|c|c|c|c|}
\hline & \multicolumn{2}{|c|}{ Grupo controle } & FC & bpm & & & \\
\hline ANIMAL & T0 & T1 & T2 & T3 & T4 & T5 & T6 \\
\hline 1 & 93 & 95 & 88 & 95 & 93 & 98 & 95 \\
\hline 2 & 105 & 100 & 89 & 78 & 117 & 128 & 120 \\
\hline 3 & 86 & 90 & 92 & 90 & 92 & 90 & 95 \\
\hline 4 & 105 & 104 & 125 & 116 & 125 & 117 & 138 \\
\hline 5 & 122 & 121 & 119 & 120 & 119 & 145 & 151 \\
\hline 6 & 92 & 90 & 95 & 96 & 98 & 106 & 108 \\
\hline 7 & 124 & 109 & 112 & 122 & 131 & 136 & 128 \\
\hline Média & 104 & 101 & 103 & 102 & 111 & 117 & 119 \\
\hline DP & 15 & 11 & 15 & 17 & 16 & 20 & 21 \\
\hline
\end{tabular}

\begin{tabular}{cccccccc}
\multicolumn{2}{c}{ Grupo HES } & FC & & & & \\
\hline ANIMAL & T0 & T1 & T2 & T3 & T4 & T5 & T6 \\
\hline $\mathbf{1}$ & 109 & 110 & 127 & 135 & 138 & 107 & 117 \\
$\mathbf{2}$ & 116 & 125 & 126 & 123 & 123 & 119 & 119 \\
$\mathbf{3}$ & 124 & 137 & 144 & 150 & 141 & 130 & 139 \\
$\mathbf{4}$ & 144 & 155 & 181 & 190 & 183 & 165 & 164 \\
$\mathbf{5}$ & 101 & 127 & 157 & 126 & 130 & 132 & 134 \\
$\mathbf{6}$ & 112 & 145 & 156 & 133 & 130 & 155 & 130 \\
$\mathbf{7}$ & 98 & 106 & 109 & 120 & 111 & 123 & 122 \\
\hline Média & 115 & 129 & 143 & 140 & 137 & 133 & 132 \\
DP & 16 & 18 & 24 & 24 & 23 & 20 & 16 \\
\hline
\end{tabular}

Grupo Solução salina FC

\begin{tabular}{cccccccc}
\hline ANIMAL & T0 & T1 & T2 & T3 & T4 & T5 & T6 \\
\hline $\mathbf{1}$ & 114 & 117 & 119 & 116 & 110 & 115 & 125 \\
$\mathbf{2}$ & 131 & 126 & 131 & 134 & 137 & 128 & 125 \\
$\mathbf{3}$ & 117 & 139 & 140 & 143 & 149 & 142 & 151 \\
$\mathbf{4}$ & 110 & 147 & 146 & 145 & 134 & 136 & 130 \\
$\mathbf{5}$ & 104 & 153 & 155 & 160 & 158 & 130 & 126 \\
$\mathbf{6}$ & 118 & 155 & 181 & 172 & 155 & 149 & 139 \\
$\mathbf{7}$ & 103 & 125 & 140 & 147 & 153 & 145 & 145 \\
\hline Média & 114 & 137 & 145 & 145 & 142 & 135 & 134 \\
DP & 10 & 15 & 20 & 18 & 17 & 12 & 11 \\
\hline
\end{tabular}




\begin{tabular}{cccccccc}
\multicolumn{1}{c}{ Grupo controle } & PAS & $\mathbf{m m H g}$ & & & \\
\hline ANIMAL & T0 & T1 & T2 & T3 & T4 & T5 & T6 \\
\hline $\mathbf{1}$ & 110 & 107 & 94 & 99 & 98 & 96 & 100 \\
$\mathbf{2}$ & 96 & 105 & 100 & 111 & 111 & 116 & 110 \\
$\mathbf{3}$ & 88 & 90 & 101 & 102 & 103 & 102 & 105 \\
$\mathbf{4}$ & 126 & 117 & 111 & 108 & 109 & 107 & 99 \\
$\mathbf{5}$ & 100 & 91 & 93 & 102 & 105 & 110 & 109 \\
$\mathbf{6}$ & 128 & 104 & 132 & 135 & 131 & 126 & 126 \\
$\mathbf{7}$ & 134 & 135 & 130 & 125 & 111 & 113 & 113 \\
\hline Média & 112 & 107 & 109 & 112 & 110 & 110 & 109 \\
DP & 17 & 14 & 15 & 12 & 10 & 9 & 8 \\
\hline
\end{tabular}

\section{Grupo HES}

\begin{tabular}{cccccccc}
\hline ANIMAL & T0 & T1 & T2 & T3 & T4 & T5 & T6 \\
\hline $\mathbf{1}$ & 113 & 115 & 119 & 117 & 129 & 131 & 137 \\
$\mathbf{2}$ & 112 & 110 & 113 & 94 & 104 & 110 & 101 \\
$\mathbf{3}$ & 102 & 120 & 122 & 124 & 132 & 132 & 128 \\
$\mathbf{4}$ & 123 & 115 & 115 & 125 & 118 & 130 & 115 \\
$\mathbf{5}$ & 106 & 111 & 115 & 100 & 100 & 107 & 105 \\
$\mathbf{6}$ & 77 & 98 & 99 & 77 & 77 & 102 & 82 \\
$\mathbf{7}$ & 86 & 101 & 89 & 95 & 94 & 110 & 97 \\
\hline Média & 103 & 110 & 110 & 105 & 108 & 117 & 109 \\
DP & 16 & 8 & 12 & 18 & 20 & 13 & 19 \\
\hline
\end{tabular}

Grupo Solução salina

PAS

\begin{tabular}{cccccccc}
\hline ANIMAL & T0 & T1 & T2 & T3 & T4 & T5 & T6 \\
\hline $\mathbf{1}$ & 123 & 128 & 125 & 115 & 108 & 126 & 120 \\
$\mathbf{2}$ & 98 & 84 & 81 & 81 & 75 & 85 & 82 \\
$\mathbf{3}$ & 103 & 101 & 88 & 95 & 96 & 109 & 100 \\
$\mathbf{4}$ & 99 & 95 & 88 & 78 & 77 & 102 & 84 \\
$\mathbf{5}$ & 108 & 94 & 88 & 96 & 95 & 113 & 105 \\
$\mathbf{6}$ & 86 & 115 & 127 & 90 & 99 & 118 & 102 \\
$\mathbf{7}$ & 95 & 103 & 97 & 99 & 94 & 104 & 101 \\
\hline Média & 102 & 103 & 99 & 93 & 92 & 108 & 99 \\
DP & 12 & 15 & 19 & 12 & 12 & 13 & 13 \\
\hline
\end{tabular}




\begin{tabular}{|c|c|c|c|c|c|c|c|}
\hline & \multicolumn{2}{|c|}{ Grupo controle } & PAD & $\mathrm{mmHs}$ & & & \\
\hline ANIMAL & T0 & T1 & T2 & T3 & T4 & T5 & T6 \\
\hline 1 & 69 & 70 & 58 & 61 & 60 & 58 & 60 \\
\hline 2 & 51 & 55 & 56 & 63 & 72 & 71 & 70 \\
\hline 3 & 53 & 57 & 66 & 67 & 69 & 68 & 73 \\
\hline 4 & 95 & 89 & 83 & 79 & 82 & 83 & 72 \\
\hline 5 & 59 & 52 & 53 & 57 & 61 & 68 & 70 \\
\hline 6 & 93 & 92 & 86 & 89 & 88 & 81 & 81 \\
\hline 7 & 103 & 100 & 97 & 92 & 74 & 84 & 86 \\
\hline Média & 75 & 74 & 71 & 73 & 72 & 73 & 73 \\
\hline DP & 22 & 20 & 17 & 14 & 10 & 10 & 8 \\
\hline
\end{tabular}

Grupo HES

\begin{tabular}{cccccccc}
\hline ANIMAL & T0 & T1 & T2 & T3 & T4 & T5 & T6 \\
\hline $\mathbf{1}$ & 77 & 74 & 78 & 79 & 84 & 95 & 95 \\
$\mathbf{2}$ & 79 & 77 & 80 & 58 & 71 & 74 & 78 \\
$\mathbf{3}$ & 69 & 77 & 75 & 85 & 91 & 92 & 96 \\
$\mathbf{4}$ & 96 & 88 & 83 & 93 & 86 & 94 & 83 \\
$\mathbf{5}$ & 70 & 73 & 76 & 61 & 64 & 76 & 74 \\
$\mathbf{6}$ & 48 & 67 & 70 & 50 & 46 & 72 & 52 \\
$\mathbf{7}$ & 52 & 61 & 50 & 57 & 56 & 75 & 60 \\
\hline Média & 70 & 74 & 73 & 69 & 71 & 83 & 77 \\
DP & 16 & 8 & 11 & 16 & 17 & 10 & 17 \\
\hline
\end{tabular}

Grupo Solução salina

PAD

\begin{tabular}{cccccccc}
\hline ANIMAL & T0 & T1 & T2 & T3 & T4 & T5 & T6 \\
\hline $\mathbf{1}$ & 84 & 84 & 76 & 76 & 72 & 80 & 75 \\
$\mathbf{2}$ & 67 & 54 & 50 & 54 & 55 & 62 & 61 \\
$\mathbf{3}$ & 67 & 62 & 51 & 58 & 60 & 73 & 64 \\
$\mathbf{4}$ & 62 & 60 & 58 & 50 & 48 & 67 & 59 \\
$\mathbf{5}$ & 71 & 50 & 48 & 58 & 56 & 70 & 66 \\
$\mathbf{6}$ & 54 & 76 & 76 & 72 & 65 & 77 & 70 \\
$\mathbf{7}$ & 54 & 59 & 54 & 60 & 58 & 63 & 62 \\
\hline Média & 66 & 64 & 59 & 61 & 59 & 70 & 65 \\
DP & 10 & 12 & 12 & 9 & 8 & 7 & 6 \\
\hline
\end{tabular}




\begin{tabular}{|c|c|c|c|c|c|c|c|}
\hline & \multicolumn{2}{|c|}{ Grupo controle } & \multirow{2}{*}{$\frac{\text { PAM }}{\text { T2 }}$} & $\mathrm{mmHg}$ & \multirow[b]{2}{*}{ T4 } & \multirow[b]{2}{*}{ T5 } & \multirow[b]{2}{*}{ T6 } \\
\hline ANIMAL & TO & T1 & & T3 & & & \\
\hline 1 & 85 & 84 & 71 & 75 & 73 & 72 & 73 \\
\hline 2 & 67 & 70 & 73 & 81 & 86 & 88 & 88 \\
\hline 3 & 66 & 70 & 79 & 81 & 82 & 80 & 85 \\
\hline 4 & 106 & 99 & 94 & 89 & 92 & 92 & 85 \\
\hline 5 & 77 & 69 & 69 & 76 & 79 & 87 & 87 \\
\hline 6 & 108 & 100 & 105 & 107 & 106 & 100 & 103 \\
\hline 7 & 115 & 112 & 108 & 105 & 89 & 97 & 98 \\
\hline Média & 89 & 86 & 86 & 88 & 87 & 88 & 88 \\
\hline DP & 20 & 18 & 17 & 13 & 11 & 10 & 10 \\
\hline
\end{tabular}

\begin{tabular}{cccccccc}
\multicolumn{2}{c}{ Grupo HES } & PAM & \multicolumn{7}{l}{} \\
\hline ANIMAL & T0 & T1 & T2 & T3 & T4 & T5 & T6 \\
\hline $\mathbf{1}$ & 93 & 91 & 91 & 91 & 99 & 110 & 111 \\
$\mathbf{2}$ & 92 & 92 & 93 & 71 & 85 & 89 & 89 \\
$\mathbf{3}$ & 84 & 95 & 96 & 102 & 108 & 107 & 110 \\
$\mathbf{4}$ & 106 & 99 & 98 & 107 & 100 & 108 & 95 \\
$\mathbf{5}$ & 84 & 90 & 95 & 78 & 81 & 91 & 88 \\
$\mathbf{6}$ & 61 & 81 & 84 & 64 & 61 & 86 & 66 \\
$\mathbf{7}$ & 65 & 77 & 68 & 73 & 73 & 90 & 77 \\
\hline Média & 84 & 89 & 89 & 84 & 87 & 97 & 91 \\
DP & 16 & 8 & 10 & 16 & 17 & 10 & 16 \\
\hline
\end{tabular}

\begin{tabular}{|c|c|c|c|c|c|c|c|}
\hline & \multicolumn{3}{|c|}{ Grupo Solução salina } & \multicolumn{2}{|l|}{ PAM } & & \\
\hline$\overline{\text { ANIMAL }}$ & T0 & T1 & T2 & T3 & T4 & T5 & T6 \\
\hline 1 & 100 & 101 & 97 & 91 & 87 & 96 & 93 \\
\hline 2 & 80 & 66 & 64 & 66 & 64 & 72 & 70 \\
\hline 3 & 82 & 80 & 69 & 76 & 78 & 89 & 80 \\
\hline 4 & 79 & 78 & 72 & 65 & 63 & 83 & 71 \\
\hline 5 & 87 & 72 & 68 & 76 & 76 & 89 & 84 \\
\hline 6 & 68 & 94 & 100 & 80 & 81 & 95 & 84 \\
\hline 7 & 70 & 79 & 75 & 80 & 74 & 83 & 80 \\
\hline Média & 81 & 81 & 78 & 76 & 75 & 87 & 80 \\
\hline DP & 11 & 12 & 15 & 9 & 9 & 8 & 8 \\
\hline
\end{tabular}




\begin{tabular}{|c|c|c|c|c|c|c|c|}
\hline & \multicolumn{2}{|c|}{ Grupo controle } & \multirow{2}{*}{$\frac{\text { PVC }}{\text { T2 }}$} & $\mathrm{mmHg}$ & \multirow[b]{2}{*}{ T4 } & \multirow[b]{2}{*}{ T5 } & \multirow[b]{2}{*}{ T6 } \\
\hline ANIMAL & TO & T1 & & T3 & & & \\
\hline 1 & 7 & 7 & 7 & 6 & 5 & 6 & 8 \\
\hline 2 & 6 & 5 & 4 & 6 & 3 & 3 & 4 \\
\hline 3 & 4 & 3 & 4 & 3 & 3 & 3 & 5 \\
\hline 4 & 3 & 3 & 5 & 3 & 3 & 3 & 2 \\
\hline 5 & 10 & 10 & 7 & 7 & 9 & 8 & 7 \\
\hline 6 & 9 & 8 & 9 & 8 & 7 & 8 & 6 \\
\hline 7 & 10 & 9 & 8 & 7 & 7 & 8 & 8 \\
\hline Média & 7,0 & 6,4 & 6,3 & 5,7 & 5,3 & 5,6 & 5,7 \\
\hline DP & 2,8 & 2,8 & 2,0 & 2,0 & 2,4 & 2,5 & 2,2 \\
\hline
\end{tabular}

\begin{tabular}{cccccccc}
\multicolumn{2}{c}{ Grupo HES } & PVC & \multicolumn{2}{c}{} \\
\hline ANIMAL & T0 & T1 & T2 & T3 & T4 & T5 & T6 \\
\hline $\mathbf{1}$ & 4 & 5 & 6 & 4 & 2 & 6 & 7 \\
$\mathbf{2}$ & 7 & 7 & 7 & 5 & 6 & 8 & 6 \\
$\mathbf{3}$ & 2 & 4 & 6 & 2 & 3 & 7 & 4 \\
$\mathbf{4}$ & 8 & 7 & 9 & 6 & 5 & 8 & 5 \\
$\mathbf{5}$ & 8 & 9 & 10 & 7 & 6 & 9 & 7 \\
$\mathbf{6}$ & 4 & 5 & 7 & 4 & 3 & 6 & 4 \\
$\mathbf{7}$ & 7 & 10 & 8 & 6 & 7 & 10 & 6 \\
\hline Média & 5,7 & 6,7 & 7,6 & 4,9 & 4,6 & 7,7 & 5,6 \\
DP & 2,4 & 2,2 & 1,5 & 1,7 & 1,9 & 1,5 & 1,3 \\
\hline
\end{tabular}

Grupo Solução salina

PVC

\begin{tabular}{cccccccc}
\hline ANIMAL & T0 & T1 & T2 & T3 & T4 & T5 & T6 \\
\hline $\mathbf{1}$ & 8 & 9 & 12 & 7 & 7 & 10 & 9 \\
$\mathbf{2}$ & 7 & 6 & 8 & 6 & 6 & 8 & 7 \\
$\mathbf{3}$ & 6 & 5 & 4 & 5 & 2 & 5 & 3 \\
$\mathbf{4}$ & 4 & 5 & 7 & 3 & 3 & 7 & 4 \\
$\mathbf{5}$ & 9 & 9 & 12 & 9 & 10 & 12 & 9 \\
$\mathbf{6}$ & 3 & 2 & 4 & 3 & 3 & 5 & 3 \\
$\mathbf{7}$ & 7 & 5 & 4 & 4 & 4 & 6 & 4 \\
\hline Média & 6,3 & 5,9 & 7,3 & 5,3 & 5,0 & 7,6 & 5,6 \\
DP & 2,1 & 2,5 & 3,6 & 2,2 & 2,8 & 2,6 & 2,7 \\
\hline
\end{tabular}




\begin{tabular}{cccccccc} 
& \multicolumn{2}{c}{ Grupo controle } & \multicolumn{2}{c}{ PAP média } & mmHg & \\
\hline ANIMAL & T0 & T1 & T2 & T3 & T4 & T5 & T6 \\
\hline $\mathbf{1}$ & 19 & 20 & 17 & 17 & 18 & 18 & 16 \\
$\mathbf{2}$ & 13 & 15 & 15 & 14 & 15 & 23 & 23 \\
$\mathbf{3}$ & 15 & 14 & 15 & 15 & 14 & 14 & 17 \\
$\mathbf{4}$ & 22 & 19 & 23 & 21 & 22 & 20 & 24 \\
$\mathbf{5}$ & 24 & 23 & 2 & 25 & 25 & 26 & 27 \\
$\mathbf{6}$ & 25 & 22 & 23 & 22 & 22 & 23 & 22 \\
$\mathbf{7}$ & 28 & 27 & 26 & 25 & 20 & 30 & 35 \\
\hline Média & 20,9 & 20,0 & 17,3 & 19,9 & 19,4 & 22,0 & 23,4 \\
DP & 5,5 & 4,5 & 8,0 & 4,6 & 4,0 & 5,3 & 6,4 \\
\hline
\end{tabular}

\begin{tabular}{cccccccc}
\multicolumn{2}{c}{ Grupo HES } & PAP & \multicolumn{7}{c}{} \\
\hline ANIMAL & T0 & T1 & T2 & T3 & T4 & T5 & T6 \\
\hline $\mathbf{1}$ & 17 & 14 & 16 & 14 & 13 & 18 & 20 \\
$\mathbf{2}$ & 17 & 18 & 17 & 13 & 14 & 18 & 14 \\
$\mathbf{3}$ & 19 & 25 & 29 & 23 & 22 & 27 & 23 \\
$\mathbf{4}$ & 22 & 20 & 20 & 36 & 31 & 38 & 29 \\
$\mathbf{5}$ & 17 & 21 & 24 & 17 & 15 & 22 & 17 \\
$\mathbf{6}$ & 15 & 17 & 18 & 14 & 16 & 21 & 17 \\
$\mathbf{7}$ & 17 & 20 & 19 & 18 & 18 & 26 & 19 \\
\hline Média & 17,7 & 19,3 & 20,4 & 19,3 & 18,4 & 24,3 & 19,9 \\
DP & 2,2 & 3,5 & 4,6 & 8,1 & 6,3 & 7,0 & 4,9 \\
\hline
\end{tabular}

\begin{tabular}{|c|c|c|c|c|c|c|c|}
\hline & \multicolumn{3}{|c|}{ Grupo Solução salina } & \multicolumn{2}{|l|}{ PAP } & & \\
\hline ANIMAL & T0 & T1 & T2 & T3 & T4 & T5 & T6 \\
\hline 1 & 14 & 16 & 19 & 15 & 14 & 23 & 17 \\
\hline 2 & 19 & 21 & 23 & 19 & 22 & 25 & 22 \\
\hline 3 & 18 & 19 & 14 & 20 & 17 & 20 & 17 \\
\hline 4 & 17 & 17 & 18 & 15 & 12 & 21 & 16 \\
\hline 5 & 19 & 26 & 20 & 26 & 24 & 30 & 25 \\
\hline 6 & 22 & 23 & 34 & 25 & 25 & 30 & 27 \\
\hline 7 & 16 & 16 & 15 & 16 & 16 & 19 & 19 \\
\hline Média & 17,9 & 19,7 & 20,4 & 19,4 & 18,6 & 24,0 & 20,4 \\
\hline DP & 2,5 & 3,8 & 6,7 & 4,6 & 5,1 & 4,5 & 4,3 \\
\hline
\end{tabular}




\begin{tabular}{|c|c|c|c|c|c|c|c|}
\hline & \multicolumn{2}{|c|}{ Grupo controle } & POAP & $\mathrm{mmHg}$ & & & \\
\hline ANIMAL & TO & T1 & T2 & T3 & T4 & T5 & T6 \\
\hline 1 & 10 & 11 & 9 & 8 & 9 & 8 & 9 \\
\hline 2 & 8 & 10 & 9 & 8 & 5 & 7 & 9 \\
\hline 3 & 6 & 7 & 7 & 6 & 6 & 6 & 7 \\
\hline 4 & 11 & 17 & 18 & 20 & 20 & 19 & 20 \\
\hline 5 & 9 & 13 & 12 & 13 & 15 & 13 & 13 \\
\hline 6 & 11 & 8 & 10 & 10 & 9 & 10 & 9 \\
\hline 7 & 14 & 13 & 10 & 11 & 10 & 10 & 11 \\
\hline Média & $\overline{9,9}$ & 11,3 & 10,7 & 10,9 & 10,6 & 10,4 & 11,1 \\
\hline DP & 2,5 & 3,4 & 3,5 & 4,6 & 5,3 & 4,4 & 4,3 \\
\hline
\end{tabular}

\section{Grupo HES}

\begin{tabular}{cccccccc}
\hline ANIMAL & T0 & T1 & T2 & T3 & T4 & T5 & T6 \\
\hline $\mathbf{1}$ & 7 & 7 & 8 & 5 & 4 & 8 & 9 \\
$\mathbf{2}$ & 7 & 8 & 8 & 5 & 6 & 9 & 5 \\
$\mathbf{3}$ & 6 & 9 & 11 & 6 & 7 & 10 & 6 \\
$\mathbf{4}$ & 11 & 13 & 11 & 8 & 7 & 7 & 6 \\
$\mathbf{5}$ & 10 & 13 & 15 & 9 & 7 & 12 & 7 \\
$\mathbf{6}$ & 9 & 10 & 11 & 7 & 7 & 11 & 8 \\
$\mathbf{7}$ & 10 & 13 & 13 & 10 & 11 & 16 & 12 \\
\hline Média & 8,6 & 10,4 & 11,0 & 7,1 & 7,0 & 10,4 & 7,6 \\
DP & 1,9 & 2,6 & 2,5 & 2,0 & 2,1 & 3,0 & 2,4 \\
\hline
\end{tabular}

Grupo Solução salina POAP

\begin{tabular}{cccccccc}
\hline ANIMAL & T0 & T1 & T2 & T3 & T4 & T5 & T6 \\
\hline $\mathbf{1}$ & 8 & 9 & 12 & 8 & 7 & 10 & 8 \\
$\mathbf{2}$ & 9 & 12 & 12 & 10 & 11 & 11 & 12 \\
$\mathbf{3}$ & 9 & 9 & 6 & 5 & 3 & 7 & 3 \\
$\mathbf{4}$ & 12 & 17 & 12 & 6 & 7 & 11 & 10 \\
$\mathbf{5}$ & 8 & 9 & 8 & 5 & 4 & 8 & 3 \\
$\mathbf{6}$ & 7 & 10 & 7 & 11 & 10 & 16 & 14 \\
$\mathbf{7}$ & 10 & 10 & 6 & 6 & 5 & 9 & 7 \\
\hline Média & 8,9 & 10,4 & 8,8 & 7,3 & 6,6 & 10,3 & 8,1 \\
DP & 1,8 & 3,0 & 2,8 & 2,3 & 2,8 & 2,7 & 3,9 \\
\hline
\end{tabular}


Grupo controle SPO2\%

\begin{tabular}{cccccccc}
\hline ANIMAL & T0 & T1 & T2 & T3 & T4 & T5 & T6 \\
\hline $\mathbf{1}$ & 99 & 98 & 98 & 99 & 100 & 100 & 100 \\
$\mathbf{2}$ & 98 & 99 & 98 & 97 & 95 & 92 & 99 \\
$\mathbf{3}$ & 96 & 100 & 92 & 91 & 92 & 98 & 97 \\
$\mathbf{4}$ & 100 & 100 & 100 & 98 & 97 & 97 & 99 \\
$\mathbf{5}$ & 96 & 98 & 98 & 98 & 98 & 94 & 97 \\
$\mathbf{6}$ & 94 & 94 & 92 & 94 & 93 & 92 & 92 \\
$\mathbf{7}$ & 97 & 97 & 97 & 97 & 97 & 96 & 100 \\
\hline Média & 97 & 98 & 96 & 96 & 96 & 96 & 98 \\
DP & $\mathbf{2}$ & 2 & 2 & 3 & 3 & 3 & 3 \\
\hline
\end{tabular}

\section{Grupo HES}

SPO2

\begin{tabular}{cccccccc}
\hline ANIMAL & T0 & T1 & T2 & T3 & T4 & T5 & T6 \\
\hline $\mathbf{1}$ & 100 & 100 & 100 & 100 & 100 & 100 & 100 \\
$\mathbf{2}$ & 100 & 100 & 100 & 100 & 100 & 100 & 100 \\
$\mathbf{3}$ & 99 & 100 & 100 & 100 & 100 & 99 & 97 \\
$\mathbf{4}$ & 98 & 97 & 98 & 100 & 100 & 100 & 100 \\
$\mathbf{5}$ & 100 & 100 & 100 & 100 & 100 & 100 & 100 \\
$\mathbf{6}$ & 100 & 100 & 100 & 100 & 100 & 100 & 100 \\
$\mathbf{7}$ & 100 & 100 & 100 & 100 & 100 & 97 & 100 \\
\hline Média & 100 & 100 & 100 & 100 & 100 & 99 & 100 \\
DP & 1 & 1 & 1 & 0 & 0 & 1 & 1 \\
\hline
\end{tabular}

Grupo Solução salina $\quad$ SPO2

\begin{tabular}{cccccccc}
\hline ANIMAL & T0 & T1 & T2 & T3 & T4 & T5 & T6 \\
\hline $\mathbf{1}$ & 100 & 100 & 100 & 100 & 100 & 100 & 100 \\
$\mathbf{2}$ & 100 & 96 & 98 & 98 & 98 & 99 & 95 \\
$\mathbf{3}$ & 98 & 100 & 100 & 100 & 100 & 100 & 96 \\
$\mathbf{4}$ & 100 & 100 & 100 & 100 & 100 & 100 & 100 \\
$\mathbf{5}$ & 99 & 99 & 99 & 100 & 100 & 100 & 100 \\
$\mathbf{6}$ & 98 & 100 & 100 & 100 & 100 & 100 & 100 \\
$\mathbf{7}$ & 99 & 98 & 100 & 99 & 100 & 100 & 100 \\
\hline Média & 99 & 99 & 100 & 100 & 100 & 100 & 99 \\
DP & 1 & 2 & 1 & 1 & 1 & 0 & 2 \\
\hline
\end{tabular}


Grupo controle IC termo

L/min/m2

\begin{tabular}{cccccccc}
\hline ANIMAL & T0 & T1 & T2 & T3 & T4 & T5 & T6 \\
\hline $\mathbf{1}$ & 4,9 & 4,5 & 4,2 & 4,4 & 3,8 & 3,5 & 3,7 \\
$\mathbf{2}$ & 5,1 & 4,6 & 4,7 & 4,8 & 4,7 & 4,5 & 4,6 \\
$\mathbf{3}$ & 4,8 & 4,6 & 4,2 & 4,6 & 4,2 & 4,1 & 4,1 \\
$\mathbf{4}$ & 5,5 & 4,8 & 5,4 & 5,3 & 5,5 & 5,1 & 5,2 \\
$\mathbf{5}$ & 5,4 & 4,8 & 4,9 & 4,8 & 4,7 & 5,2 & 5,2 \\
$\mathbf{6}$ & 4,9 & 4,6 & 5,1 & 4,6 & 4,4 & 4,4 & 4,4 \\
$\mathbf{7}$ & 5,2 & 4,1 & 4,3 & 4,8 & 5,5 & 4,9 & 4,7 \\
\hline Média & 5,12 & 4,57 & 4,68 & 4,75 & 4,68 & 4,53 & 4,55 \\
DP & 0,27 & 0,24 & 0,47 & 0,28 & 0,64 & 0,60 & 0,55 \\
\hline
\end{tabular}

Grupo HES

IC termo

\begin{tabular}{cccccccc}
\hline ANIMAL & T0 & T1 & T2 & T3 & T4 & T5 & T6 \\
\hline $\mathbf{1}$ & 5,8 & 7,7 & 9,5 & 7,7 & 7,3 & 6,6 & 7,4 \\
$\mathbf{2}$ & 5,7 & 6,6 & 7,3 & 5,5 & 6,5 & 6,8 & 5,8 \\
$\mathbf{3}$ & 5,4 & 6,7 & 7,8 & 6,1 & 5,3 & 5,7 & 5,1 \\
$\mathbf{4}$ & 6,4 & 7,8 & 9,3 & 7,6 & 7,1 & 6,4 & 6,8 \\
$\mathbf{5}$ & 5,4 & 7,2 & 9,1 & 7,5 & 6,8 & 7,4 & 6,3 \\
$\mathbf{6}$ & 4,5 & 7,3 & 8,6 & 6,7 & 6,6 & 7,4 & 5,9 \\
$\mathbf{7}$ & 5,4 & 6,8 & 8,9 & 7,8 & 7,2 & 8,3 & 7,6 \\
\hline Média & 5,51 & 7,16 & 8,64 & 6,99 & 6,69 & 6,94 & 6,41 \\
DP & 0,57 & 0,48 & 0,81 & 0,90 & 0,68 & 0,84 & 0,90 \\
\hline
\end{tabular}

Grupo Solução salina IC termo

\begin{tabular}{cccccccc}
\hline ANIMAL & T0 & T1 & T2 & T3 & T4 & T5 & T6 \\
\hline $\mathbf{1}$ & 5,3 & 5,3 & 8 & 5,3 & 5,5 & 6,3 & 7,2 \\
$\mathbf{2}$ & 5,4 & 5,4 & 5,3 & 4,3 & 4,4 & 4,5 & 4,3 \\
$\mathbf{3}$ & 5,6 & 8 & 7,5 & 7,4 & 6,4 & 6,9 & 6,8 \\
$\mathbf{4}$ & 6,3 & 8,7 & 8,6 & 6 & 5,9 & 7,7 & 7,4 \\
$\mathbf{5}$ & 4,8 & 7,5 & 7,1 & 6,8 & 5,2 & 6,9 & 6,4 \\
$\mathbf{6}$ & 5,5 & 8,1 & 7,5 & 5,6 & 4,8 & 7 & 7,3 \\
$\mathbf{7}$ & 4,3 & 5,8 & 6,9 & 6,2 & 7,1 & 7,7 & 6,4 \\
\hline Média & 5,31 & 6,97 & 7,27 & 5,94 & 5,61 & 6,71 & 6,54 \\
DP & 0,63 & 1,43 & 1,04 & 1,01 & 0,93 & 1,09 & 1,07 \\
\hline
\end{tabular}




\begin{tabular}{cccccccc} 
& \multicolumn{2}{c}{ Grupo controle } & \multicolumn{2}{c}{ IRVS termo } & \multicolumn{2}{c}{ dyn.s.m $\mathbf{I c m}^{\mathbf{5}}$} \\
\hline ANIMAL & T0 & T1 & T2 & T3 & T4 & T5 & T6 \\
\hline $\mathbf{1}$ & 1267 & 1250 & 1207 & 1263 & 1418 & 1523 & 1401 \\
$\mathbf{2}$ & 1321 & 1430 & 1621 & 1716 & 1426 & 1183 & 1300 \\
$\mathbf{3}$ & 1040 & 1153 & 1436 & 1369 & 1492 & 1518 & 1520 \\
$\mathbf{4}$ & 1500 & 1588 & 1322 & 1304 & 1296 & 1409 & 1286 \\
$\mathbf{5}$ & 986 & 987 & 1014 & 1154 & 1198 & 1211 & 1227 \\
$\mathbf{6}$ & 1604 & 1611 & 1517 & 1734 & 1782 & 1656 & 1746 \\
$\mathbf{7}$ & 1615 & 2006 & 1846 & 1648 & 1188 & 1454 & 1536 \\
\hline Média & 1333 & 1432 & 1423 & 1455 & 1400 & 1422 & 1431 \\
DP & 255 & 340 & 274 & 238 & 205 & 172 & 182 \\
\hline
\end{tabular}

\section{Grupo HES}

\begin{tabular}{cccccccc}
\hline ANIMAL & T0 & T1 & T2 & T3 & T4 & T5 & T6 \\
\hline $\mathbf{1}$ & 1229 & 890 & 712 & 901 & 1067 & 1262 & 1126 \\
$\mathbf{2}$ & 1197 & 1032 & 946 & 968 & 976 & 950 & 1146 \\
$\mathbf{3}$ & 1205 & 1092 & 926 & 1309 & 1575 & 1412 & 1659 \\
$\mathbf{4}$ & 1217 & 946 & 763 & 1069 & 1271 & 1241 & 1062 \\
$\mathbf{5}$ & 1133 & 905 & 751 & 760 & 877 & 890 & 1026 \\
$\mathbf{6}$ & 1003 & 836 & 713 & 716 & 704 & 866 & 839 \\
$\mathbf{7}$ & 855 & 787 & 537 & 690 & 730 & 771 & 752 \\
\hline Média & 1120 & 927 & 764 & 916 & 1029 & 1056 & 1087 \\
DP & 141 & 107 & 139 & 222 & 311 & 245 & 292 \\
\hline
\end{tabular}

Grupo Solução salina

\begin{tabular}{cccccccc}
\hline ANIMAL & T0 & T1 & T2 & T3 & T4 & T5 & T6 \\
\hline $\mathbf{1}$ & 1392 & 1085 & 845 & 1271 & 1160 & 1088 & 928 \\
$\mathbf{2}$ & 1081 & 889 & 847 & 1129 & 1062 & 1142 & 1185 \\
$\mathbf{3}$ & 1108 & 746 & 696 & 772 & 945 & 974 & 908 \\
$\mathbf{4}$ & 958 & 673 & 607 & 821 & 809 & 790 & 728 \\
$\mathbf{5}$ & 1299 & 676 & 635 & 792 & 1016 & 898 & 942 \\
$\mathbf{6}$ & 942 & 906 & 1024 & 1095 & 1302 & 1032 & 889 \\
$\mathbf{7}$ & 1171 & 1018 & 828 & 987 & 790 & 803 & 951 \\
\hline Média & 1136 & 856 & 783 & 981 & 1012 & 961 & 933 \\
DP & 167 & 163 & 146 & 193 & 184 & 137 & 134 \\
\hline
\end{tabular}




\begin{tabular}{cccccccc} 
& \multicolumn{2}{c}{ Grupo controle } & \multicolumn{2}{c}{ IRVP termo } & \multicolumn{2}{c}{ dyn.s. $\mathbf{m}^{\mathbf{2}} \mathbf{c m}^{\mathbf{5}}$} \\
\hline ANIMAL & T0 & T1 & T2 & T3 & T4 & T5 & T6 \\
\hline $\mathbf{1}$ & 146 & 155 & 151 & 165 & 188 & 231 & 151 \\
$\mathbf{2}$ & 108 & 130 & 141 & 137 & 172 & 223 & 230 \\
$\mathbf{3}$ & 151 & 120 & 153 & 158 & 151 & 158 & 170 \\
$\mathbf{4}$ & 163 & 182 & 185 & 184 & 177 & 229 & 235 \\
$\mathbf{5}$ & 132 & 167 & 164 & 201 & 171 & 199 & 215 \\
$\mathbf{6}$ & 227 & 245 & 205 & 210 & 234 & 234 & 234 \\
$\mathbf{7}$ & 215 & 273 & 295 & 235 & 145 & 327 & 410 \\
\hline Média & 163 & 182 & 185 & 184 & 177 & 229 & 235 \\
DP & 43 & 57 & 53 & 34 & 29 & 51 & 84 \\
\hline
\end{tabular}

Grupo HES IRVP termo

\begin{tabular}{cccccccc}
\hline ANIMAL & T0 & T1 & T2 & T3 & T4 & T5 & T6 \\
\hline $\mathbf{1}$ & 138 & 72 & 67 & 93 & 99 & 109 & 119 \\
$\mathbf{2}$ & 141 & 121 & 99 & 117 & 99 & 106 & 124 \\
$\mathbf{3}$ & 191 & 192 & 185 & 223 & 225 & 240 & 266 \\
$\mathbf{4}$ & 137 & 72 & 77 & 296 & 270 & 385 & 390 \\
$\mathbf{5}$ & 104 & 89 & 80 & 86 & 94 & 109 & 127 \\
$\mathbf{6}$ & 106 & 77 & 65 & 84 & 109 & 108 & 122 \\
$\mathbf{7}$ & 103 & 82 & 54 & 82 & 77 & 96 & 74 \\
\hline Média & 131 & 101 & 90 & 140 & 139 & 165 & 175 \\
DP & 31 & 44 & 44 & 85 & 76 & 109 & 112 \\
\hline
\end{tabular}

Grupo Solução salina IRVP termo

\begin{tabular}{cccccccc}
\hline ANIMAL & T0 & T1 & T2 & T3 & T4 & T5 & T6 \\
\hline $\mathbf{1}$ & 106 & 83 & 70 & 106 & 101 & 165 & 99 \\
$\mathbf{2}$ & 148 & 133 & 166 & 169 & 201 & 250 & 188 \\
$\mathbf{3}$ & 142 & 99 & 86 & 163 & 174 & 151 & 165 \\
$\mathbf{4}$ & 115 & 74 & 93 & 132 & 108 & 135 & 141 \\
$\mathbf{5}$ & 200 & 172 & 147 & 177 & 216 & 163 & 138 \\
$\mathbf{6}$ & 217 & 158 & 288 & 256 & 334 & 229 & 208 \\
$\mathbf{7}$ & 112 & 83 & 105 & 130 & 124 & 104 & 150 \\
\hline Média & 149 & 115 & 136 & 162 & 180 & 171 & 156 \\
DP & 44 & 40 & 75 & 49 & 82 & 51 & 36 \\
\hline
\end{tabular}


Grupo controle SVI termo $m L \cdot b^{-1} \cdot m^{-2}$

\begin{tabular}{cccccccc}
\multicolumn{1}{c}{ Grupo controle } & SVI termo & \multicolumn{3}{c}{$\mathbf{m L} \cdot \mathbf{b}^{-\mathbf{}} \cdot \mathbf{m}^{-2}$} \\
\hline ANIMAL & T0 & T1 & T2 & T3 & T4 & T5 & T6 \\
\hline $\mathbf{1}$ & 53 & 50 & 48 & 46 & 41 & 35 & 39 \\
$\mathbf{2}$ & 52 & 47 & 38 & 45 & 40 & 45 & 43 \\
$\mathbf{3}$ & 55 & 52 & 45 & 51 & 46 & 45 & 42 \\
$\mathbf{4}$ & 52 & 46 & 43 & 45 & 44 & 43 & 37 \\
$\mathbf{5}$ & 45 & 40 & 41 & 40 & 39 & 36 & 35 \\
$\mathbf{6}$ & 54 & 51 & 53 & 48 & 45 & 42 & 41 \\
$\mathbf{7}$ & 42 & 38 & 39 & 39 & 42 & 36 & 37 \\
\hline Média & 50,4 & 46,3 & 43,9 & 44,9 & 42,4 & 40,3 & 39,1 \\
DP & 4,9 & 5,4 & 5,3 & 4,2 & 2,6 & 4,5 & 3,0 \\
\hline
\end{tabular}

\begin{tabular}{cccccccc}
\multicolumn{2}{c}{ Grupo HES } & SVI & termo & & & \\
\hline ANIMAL & T0 & T1 & T2 & T3 & T4 & T5 & T6 \\
\hline $\mathbf{1}$ & 53 & 70 & 75 & 57 & 53 & 62 & 63 \\
$\mathbf{2}$ & 49 & 53 & 58 & 44 & 53 & 57 & 49 \\
$\mathbf{3}$ & 44 & 49 & 54 & 41 & 38 & 44 & 37 \\
$\mathbf{4}$ & 45 & 50 & 52 & 40 & 39 & 39 & 41 \\
$\mathbf{5}$ & 53 & 56 & 58 & 59 & 53 & 56 & 47 \\
$\mathbf{6}$ & 41 & 50 & 55 & 50 & 51 & 48 & 45 \\
$\mathbf{7}$ & 55 & 64 & 82 & 65 & 65 & 67 & 62 \\
\hline Média & 48,6 & 56,0 & 62,0 & 50,9 & 50,3 & 53,3 & 49,1 \\
DP & 5,3 & 8,1 & 11,6 & 9,7 & 9,3 & 10,0 & 9,9 \\
\hline
\end{tabular}

\begin{tabular}{|c|c|c|c|c|c|c|c|}
\hline & \multicolumn{3}{|c|}{ Grupo Solução salina } & \multirow{2}{*}{$\begin{array}{c}\text { SVI } \\
\text { T3 } \\
\end{array}$} & \multicolumn{3}{|l|}{ termo } \\
\hline ANIMAL & T0 & T1 & T2 & & T4 & T5 & T6 \\
\hline 1 & 46 & 58 & 68 & 46 & 50 & 55 & 58 \\
\hline 2 & 41 & 43 & 40 & 32 & 32 & 35 & 34 \\
\hline 3 & 48 & 58 & 53 & 51 & 43 & 49 & 45 \\
\hline 4 & 57 & 59 & 59 & 42 & 44 & 57 & 57 \\
\hline 5 & 46 & 49 & 46 & 42 & 33 & 53 & 51 \\
\hline 6 & 47 & 52 & 41 & 33 & 31 & 47 & 52 \\
\hline 7 & 42 & 47 & 49 & 42 & 46 & 53 & 44 \\
\hline Média & 46,7 & 52,3 & 50,9 & 41,1 & 39,9 & 49,9 & 48,7 \\
\hline DP & 5,2 & 6,3 & 10,1 & 6,7 & 7,7 & 7,4 & 8,4 \\
\hline
\end{tabular}




\begin{tabular}{cccccccc}
\multicolumn{2}{c}{ Grupo controle } & \multicolumn{2}{c}{ sVO2 cont. } & \% & & \\
\hline ANIMAL & T0 & T1 & T2 & T3 & T4 & T5 & T6 \\
\hline $\mathbf{1}$ & & & & & & & \\
$\mathbf{2}$ & 74 & 72 & 76 & 73 & 85 & 84 & 82 \\
$\mathbf{3}$ & & & & & & & \\
$\mathbf{4}$ & 77 & 78 & 83 & 80 & 84 & 82 & 84 \\
$\mathbf{5}$ & 78 & 72 & 72 & 69 & 78 & 77 & 78 \\
$\mathbf{6}$ & 86 & 82 & 83 & 82 & 81 & 86 & 84 \\
$\mathbf{7}$ & 87 & 86 & 80 & 78 & 81 & 80 & 79 \\
\hline Média & 80 & 78 & 79 & 76 & 82 & 82 & 81 \\
DP & 6 & 6 & 5 & 5 & 3 & 3 & 3 \\
\hline
\end{tabular}

Grupo HES SVO2 cont.

\begin{tabular}{cccccccc}
\hline ANIMAL & T0 & T1 & T2 & T3 & T4 & T5 & T6 \\
\hline $\mathbf{1}$ & 75 & 76 & 78 & 75 & 76 & 78 & 80 \\
$\mathbf{2}$ & 78 & 74 & 74 & 72 & 69 & 76 & 77 \\
$\mathbf{3}$ & & & & & & & \\
$\mathbf{4}$ & 79 & 75 & 77 & 76 & 76 & 78 & 80 \\
$\mathbf{5}$ & 82 & 82 & 80 & 79 & 79 & 85 & 83 \\
$\mathbf{6}$ & 77 & 77 & 78 & 77 & 79 & 84 & 81 \\
$\mathbf{7}$ & 79 & 73 & 60 & 62 & 64 & 75 & 78 \\
\hline Média & 78 & 76 & 75 & 74 & 74 & 79 & 80 \\
DP & 2 & 3 & 7 & 6 & 6 & 4 & 2 \\
\hline
\end{tabular}

\begin{tabular}{|c|c|c|c|c|c|c|c|}
\hline & \multicolumn{3}{|c|}{ Grupo Solução salina } & \multirow{2}{*}{$\begin{array}{c}\text { SVO2 } \\
\text { T3 }\end{array}$} & cont & \multirow[b]{2}{*}{ T5 } & \multirow[b]{2}{*}{ T6 } \\
\hline$\overline{\text { ANIMAL }}$ & T0 & T1 & T2 & & T4 & & \\
\hline 1 & 81 & 74 & 72 & 68 & 68 & 73 & 78 \\
\hline 2 & 82 & 76 & 73 & 63 & 67 & 76 & 68 \\
\hline 3 & 79 & 80 & 78 & 78 & 79 & 84 & 84 \\
\hline 4 & & & & & & & \\
\hline 5 & 75 & 72 & 66 & 68 & 72 & 77 & 75 \\
\hline 6 & 81 & 78 & 66 & 70 & 76 & 81 & 78 \\
\hline 7 & 70 & 73 & 69 & 68 & 72 & 79 & 76 \\
\hline Média & 78 & 76 & 71 & 69 & 72 & 78 & 77 \\
\hline DP & 5 & 3 & 5 & 5 & 5 & 4 & 5 \\
\hline
\end{tabular}




\begin{tabular}{cccccccc}
\multicolumn{7}{c}{ Grupo controle } & \multicolumn{2}{c}{ TEMPERAT. } & ${ }^{\circ} \mathbf{C}$ & & \\
\hline ANIMAL & T0 & T1 & T2 & T3 & T4 & T5 & T6 \\
\hline $\mathbf{1}$ & 37,1 & 37,2 & 37,5 & 38 & 38,7 & 39,2 & 39,7 \\
$\mathbf{2}$ & 38,5 & 38,7 & 39 & 39,6 & 39,4 & 39,9 & 39,5 \\
$\mathbf{3}$ & 37,3 & 37,3 & 37,4 & 37,7 & 38,2 & 38,7 & 38,5 \\
$\mathbf{4}$ & 37,4 & 37,9 & 38,3 & 38,6 & 38,5 & 38,6 & 38,6 \\
$\mathbf{5}$ & 38,1 & 38,2 & 38,5 & 38,9 & 38,9 & 38,7 & 38,7 \\
$\mathbf{6}$ & 37,5 & 37,9 & 38,1 & 38,6 & 38,9 & 38,5 & 38,4 \\
$\mathbf{7}$ & 37,6 & 38 & 38,4 & 38,7 & 38,9 & 38,9 & 38,6 \\
\hline Média & 37,6 & 37,9 & 38,2 & 38,6 & 38,8 & 38,9 & 38,8 \\
DP & 0,5 & 0,3 & 0,6 & 0,6 & 0,4 & 0,5 & 0,5 \\
\hline
\end{tabular}

Grupo HES

\begin{tabular}{cccccccc}
\hline ANIMAL & T0 & T1 & T2 & T3 & T4 & T5 & T6 \\
\hline $\mathbf{1}$ & 38,1 & 37,8 & 37,8 & 37,6 & 37,9 & 37,8 & 37,9 \\
$\mathbf{2}$ & 38,3 & 38,2 & 38 & 38,3 & 38,5 & 38,2 & 38,6 \\
$\mathbf{3}$ & 37,5 & 38,2 & 38,2 & 38,7 & 38,7 & 38,3 & 38,2 \\
$\mathbf{4}$ & 37,7 & 37,8 & 38 & 38,3 & 38,4 & 38,6 & 38,8 \\
$\mathbf{5}$ & 37,3 & 37,4 & 37,4 & 37,3 & 37,3 & 37,1 & 37,2 \\
$\mathbf{6}$ & 38,4 & 38,2 & 37,9 & 37,9 & 37,9 & 37,5 & 37,6 \\
$\mathbf{7}$ & 37,6 & 38 & 38,2 & 38,8 & 38,6 & 38,1 & 38 \\
\hline Média & 37,8 & 37,9 & 37,9 & 38,1 & 38,2 & 37,9 & 38,0 \\
DP & 0,4 & 0,3 & 0,3 & 0,6 & 0,5 & 0,5 & 0,6 \\
\hline
\end{tabular}

Grupo Solução salina

\begin{tabular}{cccccccc}
\hline ANIMAL & T0 & T1 & T2 & T3 & T4 & T5 & T6 \\
\hline $\mathbf{1}$ & 38,6 & 38,5 & 38,4 & 39,3 & 39,9 & 39,8 & 40 \\
$\mathbf{2}$ & 37,7 & 38,2 & 38,1 & 38,7 & 38,8 & 38,6 & 38,7 \\
$\mathbf{3}$ & 38 & 38,3 & 38 & 38 & 38,5 & 38,4 & 38,9 \\
$\mathbf{4}$ & 37,2 & 37,6 & 37 & 37,5 & 38 & 37,9 & 38,1 \\
$\mathbf{5}$ & 38,3 & 38,1 & 37,7 & 37,7 & 37,9 & 37,2 & 37,6 \\
$\mathbf{6}$ & 37 & 37,5 & 37,4 & 37,8 & 37,9 & 37,6 & 37,9 \\
$\mathbf{7}$ & 38,9 & 38,5 & 38,3 & 38,3 & 38,5 & 37,9 & 37,8 \\
\hline Média & 38,0 & 38,1 & 37,8 & 38,2 & 38,5 & 38,2 & 38,4 \\
DP & 0,7 & 0,4 & 0,5 & 0,6 & 0,7 & 0,8 & 0,8 \\
\hline
\end{tabular}




\begin{tabular}{cccccccc} 
& \multicolumn{2}{c}{ Grupo controle } & \multicolumn{2}{c}{ DCC termo } & L.min $^{-1}$ & & \\
\hline ANIMAL & T0 & T1 & T2 & T3 & T4 & T5 & T6 \\
\hline $\mathbf{1}$ & 4,7 & 4,5 & 4,1 & 4,1 & 4,1 & 3,7 & 3,7 \\
$\mathbf{2}$ & 3,6 & 4 & 4,2 & 3,5 & 5 & 4,8 & 4,6 \\
$\mathbf{3}$ & 4,1 & 4,4 & 4,2 & 4,1 & 4 & 4 & 4,2 \\
$\mathbf{4}$ & 5,8 & 4,8 & 5 & 5 & 4,8 & 4,6 & 4,9 \\
$\mathbf{5}$ & 5,3 & 4,9 & 4,6 & 4,8 & 4,7 & 5,4 & 5,7 \\
$\mathbf{6}$ & 4,4 & 4,2 & 4,3 & 4,2 & 3,8 & 4 & 4 \\
$\mathbf{7}$ & 5,3 & 4,1 & 4,1 & 4,8 & 4,9 & 5,6 & 4,5 \\
\hline Média & 4,7 & 4,4 & 4,4 & 4,4 & 4,5 & 4,6 & 4,5 \\
DP & 0,8 & 0,3 & 0,3 & 0,5 & 0,5 & 0,7 & 0,7 \\
\hline
\end{tabular}

Grupo HES DCC termo

\begin{tabular}{cccccccc}
\hline ANIMAL & T0 & T1 & T2 & T3 & T4 & T5 & T6 \\
\hline $\mathbf{1}$ & 5,6 & 6 & 7,7 & 7,1 & 6,7 & 6,5 & 6,3 \\
$\mathbf{2}$ & 4,8 & 5,9 & 5,2 & 5,7 & 5,4 & 5,6 & 5,6 \\
$\mathbf{3}$ & 5 & 6 & 6,2 & 5,9 & 4,5 & 4,7 & 4,9 \\
$\mathbf{4}$ & 6,5 & 5,6 & 8,8 & 5,5 & 6,9 & 6 & 6,2 \\
$\mathbf{5}$ & 5 & 7 & 8,1 & 7,8 & 7,2 & 7,5 & 7,4 \\
$\mathbf{6}$ & 5 & 6,5 & 7,8 & 6,1 & 5,6 & 6,2 & 5,7 \\
$\mathbf{7}$ & 5,3 & 6 & 6,3 & 7,1 & 6,1 & 6,3 & 7,1 \\
\hline Média & 5,3 & 6,1 & 7,2 & 6,5 & 6,1 & 6,1 & 6,2 \\
DP & 0,6 & 0,5 & 1,3 & 0,9 & 1,0 & 0,9 & 0,9 \\
\hline
\end{tabular}

Grupo Solução salina DCC termo

\begin{tabular}{cccccccc}
\hline ANIMAL & T0 & T1 & T2 & T3 & T4 & T5 & T6 \\
\hline $\mathbf{1}$ & 4,9 & 4,8 & 5,3 & 4,7 & 4,3 & 5 & 5,3 \\
$\mathbf{2}$ & 5,1 & 5,7 & 5,3 & 4,4 & 4 & 4,6 & 4,3 \\
$\mathbf{3}$ & 5,3 & 7,3 & 6,8 & 7 & 6,3 & 6,2 & 6,3 \\
$\mathbf{4}$ & 5,4 & 6,8 & 8,1 & 6 & 5,5 & 6,6 & 6,7 \\
$\mathbf{5}$ & 4,8 & 8,1 & 7,9 & 4,9 & 5,1 & 6,8 & 6,3 \\
$\mathbf{6}$ & 6,2 & 8,1 & 8,8 & 5,2 & 4,9 & 6,8 & 6,8 \\
$\mathbf{7}$ & 4,4 & 6 & 6,1 & 4,9 & 5,4 & 5,9 & 5,8 \\
\hline Média & 5,2 & 6,7 & 6,9 & 5,3 & 5,1 & 6,0 & 5,9 \\
DP & 0,6 & 1,3 & 1,4 & 0,9 & 0,8 & 0,9 & 0,9 \\
\hline
\end{tabular}




\begin{tabular}{cccccccc} 
& \multicolumn{2}{c}{ Grupo controle } & \multicolumn{2}{c}{ EDV termo } & mL & & \\
\hline ANIMAL & T0 & T1 & T2 & T3 & T4 & T5 & T6 \\
\hline $\mathbf{1}$ & 136 & 135 & 126 & 119 & 115 & 109 & 110 \\
$\mathbf{2}$ & 124 & 128 & 131 & 137 & 147 & 162 & 150 \\
$\mathbf{3}$ & 135 & 136 & 131 & 136 & 137 & 140 & 137 \\
$\mathbf{4}$ & 145 & 142 & 134 & 144 & 134 & 134 & 121 \\
$\mathbf{5}$ & 155 & 155 & 166 & 169 & 165 & 169 & 179 \\
$\mathbf{6}$ & 141 & 138 & 138 & 142 & 145 & 149 & 145 \\
$\mathbf{7}$ & 151 & 133 & 139 & 146 & 169 & 177 & 172 \\
\hline Média & 141 & 138 & 138 & 142 & 145 & 149 & 145 \\
DP & 11 & 9 & 13 & 15 & 19 & 23 & 25 \\
\hline
\end{tabular}

\begin{tabular}{cccccccc}
\multicolumn{2}{c}{ Grupo HES } & EDV & termo & & & \\
\hline ANIMAL & T0 & T1 & T2 & T3 & T4 & T5 & T6 \\
\hline $\mathbf{1}$ & 130 & 127 & 135 & 128 & 124 & 131 & 170 \\
$\mathbf{2}$ & 156 & 153 & 147 & 143 & 141 & 147 & 145 \\
$\mathbf{3}$ & 103 & 117 & 118 & 115 & 106 & 113 & 106 \\
$\mathbf{4}$ & 163 & 170 & 176 & 176 & 178 & 163 & 161 \\
$\mathbf{5}$ & 127 & 121 & 115 & 112 & 109 & 105 & 109 \\
$\mathbf{6}$ & 143 & 154 & 164 & 147 & 143 & 148 & 153 \\
$\mathbf{7}$ & 172 & 170 & 167 & 173 & 156 & 160 & 167 \\
\hline Média & 142 & 145 & 146 & 142 & 137 & 138 & 144 \\
DP & 24 & 23 & 24 & 26 & 26 & 23 & 27 \\
\hline
\end{tabular}

Grupo Solução salina EDV termo

\begin{tabular}{cccccccc}
\hline ANIMAL & T0 & T1 & T2 & T3 & T4 & T5 & T6 \\
\hline $\mathbf{1}$ & & & & & & & \\
$\mathbf{2}$ & 137 & 174 & 169 & 131 & 168 & 151 & 160 \\
$\mathbf{3}$ & 139 & 160 & 158 & 154 & 143 & 149 & 142 \\
$\mathbf{4}$ & & & & & & & \\
$\mathbf{5}$ & 123 & 144 & 138 & 95 & 98 & 150 & 130 \\
$\mathbf{6}$ & 152 & 150 & 154 & 127 & 114 & 163 & 159 \\
$\mathbf{7}$ & 136 & 135 & 127 & 121 & 120 & 143 & 127 \\
\hline Média & 137 & 153 & 149 & 126 & 129 & 151 & 144 \\
DP & 10 & 15 & 17 & 21 & 27 & 7 & 16 \\
\hline
\end{tabular}




\begin{tabular}{|c|c|c|c|c|c|c|c|}
\hline & \multicolumn{2}{|c|}{ Grupo controle } & \multicolumn{2}{|c|}{ EF termo } & \multicolumn{2}{|l|}{$\%$} & \multirow[b]{2}{*}{ T6 } \\
\hline ANIMAL & T0 & T1 & T2 & T3 & T4 & T5 & \\
\hline 1 & 38 & 35 & 36 & 36 & 29 & 26 & 27 \\
\hline 2 & 24 & 27 & 26 & 33 & 29 & 29 & 30 \\
\hline 3 & 37 & 29 & 24 & 26 & 25 & 27 & 30 \\
\hline 4 & 36 & 33 & 35 & 32 & 31 & 29 & 30 \\
\hline 5 & 29 & 26 & 23 & 24 & 24 & 22 & 21 \\
\hline 6 & 23 & 21 & 20 & 22 & 18 & 17 & 20 \\
\hline 7 & 29 & 29 & 26 & 27 & 23 & 23 & 22 \\
\hline Média & 31 & 29 & 27 & 29 & 26 & 25 & 26 \\
\hline DP & 6 & 5 & 6 & 5 & 4 & 4 & 5 \\
\hline
\end{tabular}

Grupo HES

EF termo

\begin{tabular}{cccccccc}
\hline ANIMAL & T0 & T1 & T2 & T3 & T4 & T5 & T6 \\
\hline $\mathbf{1}$ & 40 & 44 & 46 & 43 & 41 & 37 & 31 \\
$\mathbf{2}$ & 27 & 32 & 30 & 31 & 29 & 31 & 32 \\
$\mathbf{3}$ & 39 & 38 & 37 & 35 & 29 & 30 & 32 \\
$\mathbf{4}$ & 28 & 26 & 30 & 23 & & 22 & 23 \\
$\mathbf{5}$ & 40 & 45 & 53 & 49 & 51 & 49 & 47 \\
$\mathbf{6}$ & 30 & 30 & 32 & 30 & 31 & 28 & 27 \\
$\mathbf{7}$ & 31 & 34 & 36 & 34 & 36 & 33 & 34 \\
\hline Média & 34 & 36 & 38 & 35 & 36 & 33 & 32 \\
DP & 6 & 7 & 9 & 9 & 9 & 8 & 7 \\
\hline
\end{tabular}

Grupo Solução salina EF termo

\begin{tabular}{cccccccc}
\hline ANIMAL & T0 & T1 & T2 & T3 & T4 & T5 & T6 \\
\hline $\mathbf{1}$ & 40 & 39 & 42 & 40 & 38 & 37 & 37 \\
$\mathbf{2}$ & 27 & 25 & 24 & 25 & 47 & 23 & 20 \\
$\mathbf{3}$ & 33 & 33 & 31 & 32 & 30 & 29 & 29 \\
$\mathbf{4}$ & & & & & & & \\
$\mathbf{5}$ & 36 & 41 & 39 & 32 & 33 & 36 & 36 \\
$\mathbf{6}$ & 33 & 36 & 35 & 29 & 29 & 28 & 29 \\
$\mathbf{7}$ & 32 & 36 & 36 & 28 & 29 & 29 & 32 \\
\hline Média & 34 & 35 & 35 & 31 & 34 & 30 & 31 \\
DP & 4 & 6 & 6 & 5 & 7 & 5 & 6 \\
\hline
\end{tabular}




\begin{tabular}{cccccccc} 
& \multicolumn{2}{c}{ Grupo controle } & \multicolumn{2}{c}{ EDVI termo } & $\mathbf{~ m L . ~}^{-2}$ & & \\
\hline ANIMAL & T0 & T1 & T2 & T3 & T4 & T5 & T6 \\
\hline $\mathbf{1}$ & 158 & 153 & 147 & 137 & 133 & 126 & 128 \\
$\mathbf{2}$ & 149 & 154 & 145 & 152 & 164 & 180 & 180 \\
$\mathbf{3}$ & 162 & 163 & 164 & 158 & 163 & 165 & 145 \\
$\mathbf{4}$ & 159 & 156 & 147 & 158 & 147 & 148 & 133 \\
$\mathbf{5}$ & 169 & 169 & 180 & 183 & 180 & 184 & 195 \\
$\mathbf{6}$ & & & & & & & \\
$\mathbf{7}$ & 159 & 138 & 145 & 152 & 177 & 184 & 179 \\
\hline Média & 159 & 156 & 155 & 157 & 161 & 165 & 160 \\
DP & 6 & 11 & 14 & 15 & 18 & 23 & 28 \\
\hline
\end{tabular}

\section{Grupo HES}

\begin{tabular}{cccccccc}
\hline ANIMAL & T0 & T1 & T2 & T3 & T4 & T5 & T6 \\
\hline $\mathbf{1}$ & 148 & 144 & 154 & 146 & 141 & 149 & 193 \\
$\mathbf{2}$ & 178 & 174 & 167 & 162 & 160 & 167 & 165 \\
$\mathbf{3}$ & 114 & 130 & 131 & 128 & 118 & 126 & 118 \\
$\mathbf{4}$ & 181 & 188 & 195 & 175 & 160 & 181 & 179 \\
$\mathbf{5}$ & 134 & 127 & 121 & 118 & 115 & 111 & 115 \\
$\mathbf{6}$ & 162 & 175 & 186 & 167 & 163 & 168 & 174 \\
$\mathbf{7}$ & 183 & 181 & 177 & 184 & 166 & 170 & 177 \\
\hline Média & 157 & 160 & 162 & 154 & 146 & 153 & 160 \\
DP & 26 & 25 & 28 & 25 & 22 & 26 & 31 \\
\hline
\end{tabular}

Grupo Solução salina EDVI termo

\begin{tabular}{cccccccc}
\hline ANIMAL & T0 & T1 & T2 & T3 & T4 & T5 & T6 \\
\hline $\mathbf{1}$ & & & & & & & \\
$\mathbf{2}$ & 158 & 200 & 195 & 150 & 194 & 174 & 184 \\
$\mathbf{3}$ & 152 & 184 & 181 & 177 & 164 & 172 & 163 \\
$\mathbf{4}$ & & & & & & & \\
$\mathbf{5}$ & 121 & 142 & 133 & 94 & 97 & 147 & 127 \\
$\mathbf{6}$ & 158 & 156 & 161 & 116 & 119 & 169 & 166 \\
$\mathbf{7}$ & 158 & 156 & 147 & 140 & 139 & 166 & 147 \\
\hline Média & 149 & 168 & 163 & 135 & 143 & 166 & 157 \\
DP & 16 & 24 & 25 & 32 & 38 & 11 & 21 \\
\hline
\end{tabular}




\begin{tabular}{cccccccc}
\multicolumn{1}{c}{ Grupo controle } & \multicolumn{2}{c}{ ESV termo } & mL & & \\
\hline ANIMAL & T0 & T1 & T2 & T3 & T4 & T5 & T6 \\
\hline $\mathbf{1}$ & 85 & 83 & 81 & 75 & 82 & 80 & 80 \\
$\mathbf{2}$ & 94 & 97 & 96 & 91 & 105 & 115 & 120 \\
$\mathbf{3}$ & 85 & 97 & 103 & 96 & 101 & 99 & 105 \\
$\mathbf{4}$ & 92 & 95 & 88 & 97 & 92 & 95 & 84 \\
$\mathbf{5}$ & 110 & 114 & 128 & 129 & 126 & 131 & 141 \\
$\mathbf{6}$ & 161 & 180 & 175 & 155 & 175 & 178 & 152 \\
$\mathbf{7}$ & 109 & 95 & 103 & 107 & 132 & 136 & 134 \\
\hline Média & 105 & 109 & 111 & 107 & 116 & 119 & 117 \\
DP & 27 & 33 & 32 & 27 & 31 & 33 & 28 \\
\hline
\end{tabular}

\begin{tabular}{cccccccc}
\multicolumn{2}{l}{ Grupo HES } & ESV & termo & & & \\
\hline ANIMAL & T0 & T1 & T2 & T3 & T4 & T5 & T6 \\
\hline $\mathbf{1}$ & 78 & 70 & 72 & 74 & 74 & 82 & 118 \\
$\mathbf{2}$ & 114 & 105 & 103 & 98 & 100 & 100 & 99 \\
$\mathbf{3}$ & 63 & 73 & 74 & 75 & 75 & 79 & 72 \\
$\mathbf{4}$ & 117 & 125 & 123 & 121 & & 126 & 124 \\
$\mathbf{5}$ & 77 & 66 & 55 & 57 & 53 & 54 & 58 \\
$\mathbf{6}$ & 100 & 108 & 112 & 103 & 99 & 106 & 112 \\
$\mathbf{7}$ & 119 & 112 & 107 & 114 & 9 & 107 & 111 \\
\hline Média & 95 & 94 & 92 & 92 & 68 & 93 & 99 \\
DP & 23 & 24 & 25 & 24 & 34 & 24 & 25 \\
\hline
\end{tabular}

\begin{tabular}{|c|c|c|c|c|c|c|c|}
\hline & \multicolumn{3}{|c|}{ Grupo Solução salina } & \multirow{2}{*}{$\frac{\text { ESV }}{\text { T3 }}$} & termo & \multirow[b]{2}{*}{ T5 } & \multirow[b]{2}{*}{ T6 } \\
\hline ANIMAL & T0 & $\mathrm{T} 1$ & $\mathrm{~T} 2$ & & T4 & & \\
\hline 1 & 62 & 67 & 65 & 62 & 63 & 73 & 72 \\
\hline 2 & 101 & 131 & 128 & 98 & 90 & 116 & 128 \\
\hline 3 & 89 & 107 & 108 & 105 & 101 & 106 & 100 \\
\hline 4 & & & & & & & \\
\hline 5 & 79 & 85 & 83 & 65 & 65 & 96 & 83 \\
\hline 6 & 101 & 96 & 100 & 79 & 81 & 117 & 113 \\
\hline 7 & 93 & 87 & 81 & 87 & 85 & 101 & 87 \\
\hline Média & 88 & 96 & 94 & 83 & 81 & 102 & 97 \\
\hline DP & 15 & 22 & 22 & 17 & 15 & 16 & 21 \\
\hline
\end{tabular}




\begin{tabular}{cccccccc} 
& \multicolumn{1}{c}{ Grupo controle } & sV termo & \multicolumn{5}{c}{ mL.bat $^{-1}$} \\
\hline ANIMAL & T0 & T1 & T2 & T3 & T4 & T5 & T6 \\
\hline $\mathbf{1}$ & 51 & 49 & 46 & 43 & 33 & 28 & 30 \\
$\mathbf{2}$ & 29 & 30 & 34 & 46 & 43 & 47 & 45 \\
$\mathbf{3}$ & 50 & 38 & 33 & 35 & 34 & 37 & 39 \\
$\mathbf{4}$ & 52 & 47 & 47 & 46 & 41 & 39 & 37 \\
$\mathbf{5}$ & 45 & 41 & 38 & 40 & 39 & 38 & 38 \\
$\mathbf{6}$ & 48 & 48 & 44 & 43 & 38 & 37 & 38 \\
$\mathbf{7}$ & 44 & 38 & 37 & 39 & 38 & 41 & 38 \\
\hline Média & 46 & 42 & 40 & 42 & 38 & 38 & 38 \\
DP & 8 & 7 & 6 & 4 & 4 & 6 & 4 \\
\hline
\end{tabular}

Grupo HES SV termo

\begin{tabular}{cccccccc}
\hline ANIMAL & T0 & T1 & T2 & T3 & T4 & T5 & T6 \\
\hline $\mathbf{1}$ & 52 & 56 & 62 & 55 & 51 & 49 & 52 \\
$\mathbf{2}$ & 42 & 49 & 44 & 44 & 41 & 46 & 46 \\
$\mathbf{3}$ & 40 & 44 & 44 & 40 & 31 & 34 & 34 \\
$\mathbf{4}$ & 45 & 45 & 53 & 37 & & 37 & 37 \\
$\mathbf{5}$ & 50 & 55 & 60 & 55 & 56 & 52 & 51 \\
$\mathbf{6}$ & 43 & 46 & 52 & 45 & 44 & 41 & 41 \\
$\mathbf{7}$ & 53 & 58 & 60 & 59 & 57 & 52 & 56 \\
\hline Média & 46 & 50 & 54 & 48 & 47 & 44 & 45 \\
DP & 5 & 6 & 8 & 8 & 10 & 7 & 8 \\
\hline
\end{tabular}

Grupo Solução salina SV termo

\begin{tabular}{cccccccc}
\hline ANIMAL & T0 & T1 & T2 & T3 & T4 & T5 & T6 \\
\hline $\mathbf{1}$ & 41 & 43 & 47 & 41 & 38 & 43 & 42 \\
$\mathbf{2}$ & 37 & 43 & 41 & 33 & 79 & 35 & 33 \\
$\mathbf{3}$ & 44 & 53 & 49 & 49 & 42 & 44 & 42 \\
$\mathbf{4}$ & 47 & 47 & 55 & 43 & 42 & 50 & 52 \\
$\mathbf{5}$ & 44 & 59 & 53 & 31 & 33 & 54 & 46 \\
$\mathbf{6}$ & 51 & 54 & 54 & 32 & 33 & 46 & 46 \\
$\mathbf{7}$ & 44 & 49 & 46 & 34 & 35 & 41 & 40 \\
\hline Média & 44 & 50 & 49 & 38 & 43 & 45 & 43 \\
DP & 4 & 6 & 5 & 7 & 16 & 6 & 6 \\
\hline
\end{tabular}


Grupo controle

dp max

\begin{tabular}{cccccccc}
\hline ANIMAL & T0 & T1 & T2 & T3 & T4 & T5 & T6 \\
\hline $\mathbf{1}$ & 395 & 380 & 375 & 389 & 370 & 425 & 444 \\
$\mathbf{2}$ & 308 & 320 & 330 & 356 & 435 & 461 & 470 \\
$\mathbf{3}$ & 275 & 299 & 288 & 303 & 309 & 265 & 270 \\
$\mathbf{4}$ & 230 & 218 & 220 & 230 & 231 & 228 & 244 \\
$\mathbf{5}$ & 330 & 335 & 350 & 383 & 390 & 399 & 404 \\
$\mathbf{6}$ & 236 & 390 & 398 & 386 & 394 & 415 & 440 \\
$\mathbf{7}$ & 259 & 314 & 304 & 304 & 345 & 253 & 254 \\
\hline Média & 290 & 322 & 324 & 336 & 353 & 349 & 361 \\
DP & 59 & 57 & 60 & 60 & 67 & 97 & 100 \\
\hline
\end{tabular}

Grupo HES

dp max

\begin{tabular}{cccccccc}
\hline ANIMAL & T0 & T1 & T2 & T3 & T4 & T5 & T6 \\
\hline $\mathbf{1}$ & 415 & 426 & 289 & 438 & 395 & 431 & 528 \\
$\mathbf{2}$ & 259 & 294 & 319 & 294 & 314 & 291 & 270 \\
$\mathbf{3}$ & 374 & 460 & 526 & 444 & 479 & 479 & 443 \\
$\mathbf{4}$ & 263 & 286 & 348 & 406 & 420 & 361 & 419 \\
$\mathbf{5}$ & 294 & 333 & 356 & 344 & 343 & 283 & 268 \\
$\mathbf{6}$ & 223 & 266 & 288 & 248 & 269 & 260 & 228 \\
$\mathbf{7}$ & 221 & 265 & 308 & 288 & 235 & 279 & 280 \\
\hline Média & 293 & 333 & 348 & 352 & 351 & 341 & 348 \\
DP & 75 & 79 & 83 & 79 & 86 & 85 & 114 \\
\hline
\end{tabular}

Grupo Solução salina dp $\max$

\begin{tabular}{cccccccc}
\hline ANIMAL & T0 & T1 & T2 & T3 & T4 & T5 & T6 \\
\hline $\mathbf{1}$ & 291 & 390 & 345 & 316 & 293 & 341 & 388 \\
$\mathbf{2}$ & 273 & 274 & 261 & 331 & 256 & 211 & 205 \\
$\mathbf{3}$ & 293 & 320 & 293 & 316 & 334 & 331 & 355 \\
$\mathbf{4}$ & 278 & 305 & 256 & 251 & 260 & 281 & \\
$\mathbf{5}$ & 341 & 458 & 526 & 506 & 484 & 455 & 404 \\
$\mathbf{6}$ & 274 & 330 & 451 & 223 & 333 & 344 & 244 \\
$\mathbf{7}$ & 299 & 321 & 369 & 354 & 325 & 358 & 340 \\
\hline Média & 293 & 343 & 357 & 328 & 326 & 332 & 323 \\
DP & 24 & 62 & 101 & 91 & 77 & 74 & 80 \\
\hline
\end{tabular}




\begin{tabular}{cccccccc}
\multicolumn{7}{c}{ Grupo controle } & V V S \% \\
\hline ANIMAL & T0 & T1 & T2 & T3 & T4 & T5 & T6 \\
\hline $\mathbf{1}$ & 8 & 9 & 10 & 8 & 9 & 7 & 8 \\
$\mathbf{2}$ & 9 & 9 & 8 & 13 & 7 & 7 & 8 \\
$\mathbf{3}$ & 11 & 13 & 13 & 14 & 13 & 13 & 12 \\
$\mathbf{4}$ & 11 & 8 & 9 & 8 & 10 & 12 & 13 \\
$\mathbf{5}$ & 10 & 7 & 7 & 7 & 8 & 9 & 8 \\
$\mathbf{6}$ & 9 & 9 & 7 & 8 & 8 & 8 & 10 \\
$\mathbf{7}$ & 8 & 7 & 8 & 10 & 11 & 12 & 12 \\
\hline Média & 9,4 & 8,9 & 8,9 & 9,7 & 9,4 & 9,7 & 10,1 \\
DP & 1,3 & 2,0 & 2,1 & 2,8 & 2,1 & 2,6 & 2,2 \\
\hline
\end{tabular}

\begin{tabular}{cccccccc}
\multicolumn{2}{c}{ Grupo HES } & \multicolumn{2}{c}{ V V S } \\
\hline ANIMAL & T0 & T1 & T2 & T3 & T4 & T5 & T6 \\
\hline $\mathbf{1}$ & 9 & 11 & 16 & 11 & 17 & 16 & 10 \\
$\mathbf{2}$ & 12 & 8 & 9 & 10 & 10 & 6 & 19 \\
$\mathbf{3}$ & 6 & 8 & 6 & 16 & 8 & 5 & 9 \\
$\mathbf{4}$ & 10 & 10 & 10 & 16 & 19 & 11 & 19 \\
$\mathbf{5}$ & 6 & 6 & 6 & 8 & 9 & 6 & 9 \\
$\mathbf{6}$ & 7 & 10 & 10 & 12 & 11 & 9 & 9 \\
$\mathbf{7}$ & 7 & 5 & 6 & 11 & 13 & 8 & 8 \\
\hline Média & 8,1 & 8,3 & 9,0 & 12,0 & 12,4 & 8,7 & 11,9 \\
DP & 2,3 & 2,2 & 3,6 & 3,0 & 4,2 & 3,8 & 4,9 \\
\hline
\end{tabular}

\section{Grupo Solução salina}

\begin{tabular}{cccccccc}
\hline ANIMAL & T0 & T1 & T2 & T3 & T4 & T5 & T6 \\
\hline $\mathbf{1}$ & 10 & 7 & 11 & 18 & 16 & 7 & 6 \\
$\mathbf{2}$ & 15 & 11 & 11 & 21 & 17 & 16 & 28 \\
$\mathbf{3}$ & 11 & 8 & 11 & 11 & 11 & 9 & 10 \\
$\mathbf{4}$ & 7 & 8 & 7 & 12 & 12 & 5 & 8 \\
$\mathbf{5}$ & 8 & 14 & 17 & 20 & 21 & 11 & 17 \\
$\mathbf{6}$ & 12 & 10 & 16 & 18 & 19 & 12 & 16 \\
$\mathbf{7}$ & 10 & 8 & 12 & 13 & 14 & 10 & 9 \\
\hline Média & 10,4 & 9,4 & 12,1 & 16,1 & 15,7 & 10,0 & 14,3 \\
DP & 2,6 & 2,4 & 3,4 & 4,1 & 3,6 & 3,6 & 7,9 \\
\hline
\end{tabular}


Grupo controle CEVAP $\mathrm{mL}$

\begin{tabular}{cccccccc}
\hline ANIMAL & T0 & T1 & T2 & T3 & T4 & T5 & T6 \\
\hline $\mathbf{1}$ & 254 & 255 & 258 & 253 & 255 & 330 & 341 \\
$\mathbf{2}$ & 285 & 280 & 281 & 282 & 284 & 248 & 260 \\
$\mathbf{3}$ & 319 & 289 & 323 & 294 & 310 & 248 & 270 \\
$\mathbf{4}$ & 276 & 274 & 281 & 280 & 271 & 284 & 258 \\
$\mathbf{5}$ & 279 & 296 & 319 & 335 & 364 & 339 & 331 \\
$\mathbf{6}$ & 236 & 266 & 271 & 309 & 315 & 315 & 288 \\
$\mathbf{7}$ & 338 & 332 & 330 & 364 & 400 & 374 & 371 \\
\hline Média & 283,9 & 284,6 & 294,7 & 302,4 & 314,1 & 305,4 & 302,7 \\
DP & 35,2 & 25,0 & 28,6 & 37,3 & 51,9 & 47,6 & 44,8 \\
\hline
\end{tabular}

\section{Grupo HES CEVAP}

\begin{tabular}{cccccccc}
\hline ANIMAL & T0 & T1 & T2 & T3 & T4 & T5 & T6 \\
\hline $\mathbf{1}$ & 315 & 328 & 293 & 339 & 321 & 281 & 331 \\
$\mathbf{2}$ & 265 & 267 & 256 & 263 & 274 & 287 & 266 \\
$\mathbf{3}$ & 255 & 277 & 275 & 297 & 316 & 316 & 281 \\
$\mathbf{4}$ & 334 & 353 & 374 & 326 & 319 & 340 & 363 \\
$\mathbf{5}$ & 282 & 293 & 314 & 314 & 303 & 314 & 322 \\
$\mathbf{6}$ & 313 & 326 & 343 & 359 & 360 & 424 & 408 \\
$\mathbf{7}$ & 352 & 347 & 358 & 337 & 349 & 329 & 329 \\
\hline Média & 302,3 & 313,0 & 316,1 & 319,3 & 320,3 & 327,3 & 328,6 \\
DP & 36,0 & 34,1 & 44,1 & 31,7 & 28,5 & 47,6 & 47,8 \\
\hline
\end{tabular}

Grupo Solução salina CEVAP

\begin{tabular}{cccccccc}
\hline ANIMAL & T0 & T1 & T2 & T3 & T4 & T5 & T6 \\
\hline $\mathbf{1}$ & 276 & 271 & 257 & 236 & 236 & 270 & 272 \\
$\mathbf{2}$ & 296 & 278 & 287 & 266 & 247 & 253 & 254 \\
$\mathbf{3}$ & 333 & 266 & 307 & 315 & 320 & 335 & 315 \\
$\mathbf{4}$ & 343 & 325 & 315 & 305 & 301 & 342 & 358 \\
$\mathbf{5}$ & 418 & 414 & 417 & 410 & 409 & 495 & 508 \\
$\mathbf{6}$ & 447 & 474 & 468 & 468 & 454 & 503 & 543 \\
$\mathbf{7}$ & 263 & 265 & 248 & 271 & 283 & 283 & 259 \\
\hline Média & 339,4 & 327,6 & 328,4 & 324,4 & 321,4 & 354,4 & 358,4 \\
DP & 70,2 & 83,9 & 82,9 & 84,2 & 81,6 & 104,0 & 120,1 \\
\hline
\end{tabular}




\begin{tabular}{cccccccc}
\multicolumn{1}{c}{ Grupo controle } & VSIT & mL & & & \\
\hline ANIMAL & T0 & T1 & T2 & T3 & T4 & T5 & T6 \\
\hline $\mathbf{1}$ & 707 & 680 & 657 & 679 & 637 & 728 & 750 \\
$\mathbf{2}$ & 839 & 820 & 775 & 733 & 721 & 793 & 805 \\
$\mathbf{3}$ & 808 & 872 & 791 & 861 & 781 & 857 & 780 \\
$\mathbf{4}$ & 842 & 817 & 828 & 759 & 722 & 690 & 654 \\
$\mathbf{5}$ & 724 & 715 & 701 & 701 & 710 & 670 & 700 \\
$\mathbf{6}$ & 641 & 632 & 618 & 628 & 626 & 626 & 613 \\
$\mathbf{7}$ & 790 & 733 & 702 & 686 & 674 & 680 & 651 \\
\hline Média & 764,4 & 752,7 & 724,6 & 721,0 & 695,9 & 720,6 & 707,6 \\
DP & 75,6 & 86,2 & 76,0 & 74,4 & 54,1 & 79,6 & 72,6 \\
\hline
\end{tabular}

\section{Grupo HES}

VSIT

\begin{tabular}{cccccccc}
\hline ANIMAL & T0 & T1 & T2 & T3 & T4 & T5 & T6 \\
\hline $\mathbf{1}$ & 816 & 869 & 968 & 780 & 745 & 894 & 767 \\
$\mathbf{2}$ & 735 & 767 & 798 & 708 & 745 & 792 & 746 \\
$\mathbf{3}$ & 652 & 699 & 737 & 650 & 622 & 662 & 613 \\
$\mathbf{4}$ & 801 & 851 & 871 & 741 & 693 & 778 & 734 \\
$\mathbf{5}$ & 778 & 830 & 908 & 821 & 762 & 834 & 750 \\
$\mathbf{6}$ & 748 & 811 & 858 & 755 & 754 & 798 & 774 \\
$\mathbf{7}$ & 754 & 878 & 867 & 746 & 749 & 810 & 777 \\
\hline Média & 754,9 & 815,0 & 858,1 & 743,0 & 724,3 & 795,4 & 737,3 \\
DP & 53,9 & 63,5 & 74,3 & 54,0 & 50,4 & 70,2 & 57,0 \\
\hline
\end{tabular}

Grupo Solução

\begin{tabular}{cccccccc}
\multicolumn{7}{c}{ salina } & VSIT \\
\hline ANIMAL & T0 & T1 & T2 & T3 & T4 & T5 & T6 \\
\hline $\mathbf{1}$ & 645 & 661 & 678 & 601 & 595 & 631 & 643 \\
$\mathbf{2}$ & 612 & 639 & 620 & 554 & 550 & 598 & 589 \\
$\mathbf{3}$ & 754 & 700 & 694 & 692 & 676 & 712 & 705 \\
$\mathbf{4}$ & 744 & 734 & 749 & 630 & 616 & 740 & 718 \\
$\mathbf{5}$ & 866 & 831 & 789 & 765 & 689 & 862 & 856 \\
$\mathbf{6}$ & 920 & 936 & 767 & 765 & 795 & 871 & 852 \\
$\mathbf{7}$ & 739 & 733 & 638 & 585 & 539 & 620 & 626 \\
\hline Média & 754,3 & 747,7 & 705,0 & 656,0 & 637,1 & 719,1 & 712,7 \\
DP & 109,9 & 103,6 & 65,1 & 85,8 & 90,0 & 112,7 & 106,2 \\
\hline
\end{tabular}


Grupo controle IC transpulmonar $\quad$ L. $\min ^{-1} \cdot \mathrm{m}^{-2}$

\begin{tabular}{cccccccc}
\hline ANIMAL & T0 & T1 & T2 & T3 & T4 & T5 & T6 \\
\hline $\mathbf{1}$ & 5,1 & 4,7 & 4,21 & 4,6 & 4,2 & 4,44 & 4,59 \\
$\mathbf{2}$ & 4,66 & 4,3 & 4,06 & 5,1 & 4,76 & 6,32 & 6,2 \\
$\mathbf{3}$ & 5,71 & 6,01 & 5,2 & 5,52 & 5,04 & 5,42 & 5,2 \\
$\mathbf{4}$ & 5,68 & 5,24 & 5,8 & 5,47 & 5,58 & 5,11 & 5,37 \\
$\mathbf{5}$ & 5,64 & 5,05 & 5,04 & 5,11 & 5,15 & 5,35 & 5,6 \\
$\mathbf{6}$ & 5,19 & 4,62 & 4,91 & 4,63 & 4,74 & 4,74 & 5,04 \\
$\mathbf{7}$ & 5,23 & 4,33 & 4,5 & 4,81 & 5,15 & 4,94 & 4,65 \\
\hline Média & 5,3 & 4,9 & 4,8 & 5,0 & 4,9 & 5,2 & 5,2 \\
DP & 0,4 & 0,6 & 0,6 & 0,4 & 0,4 & 0,6 & 0,6 \\
\hline
\end{tabular}

Grupo HES

IC transpulmonar

\begin{tabular}{cccccccc}
\hline ANIMAL & T0 & T1 & T2 & T3 & T4 & T5 & T6 \\
\hline $\mathbf{1}$ & 5,86 & 7,23 & 9,27 & 7,09 & 6,57 & 6,91 & 6,61 \\
$\mathbf{2}$ & 5,69 & 6,65 & 7,39 & 5,75 & 6,6 & 6,94 & 6,11 \\
$\mathbf{3}$ & 5,5 & 6,54 & 7,47 & 6,09 & 5,53 & 5,64 & 5,28 \\
$\mathbf{4}$ & 6,03 & 7,25 & 8,32 & 7,09 & 6,62 & 6,54 & 6,88 \\
$\mathbf{5}$ & 5,57 & 6,85 & 8,31 & 7,23 & 6,65 & 6,91 & 5,73 \\
$\mathbf{6}$ & 5,08 & 7,08 & 8,22 & 6,48 & 6,38 & 7,18 & 6,18 \\
$\mathbf{7}$ & 5,7 & 6,73 & 8,01 & 6,85 & 6,49 & 7,53 & 7,07 \\
\hline Média & 5,6 & 6,9 & 8,1 & 6,7 & 6,4 & 6,8 & 6,3 \\
DP & 0,3 & 0,3 & 0,6 & 0,6 & 0,4 & 0,6 & 0,6 \\
\hline
\end{tabular}

Grupo Solução salina

IC transpulmonar

\begin{tabular}{cccccccc}
\hline ANIMAL & T0 & T1 & T2 & T3 & T4 & T5 & T6 \\
\hline $\mathbf{1}$ & 5,17 & 6,13 & 7,33 & 5,22 & 5,24 & 5,94 & 6,79 \\
$\mathbf{2}$ & 5,13 & 5,46 & 5,45 & 4,36 & 4,52 & 4,89 & 4,63 \\
$\mathbf{3}$ & 5,67 & 7,63 & 7,68 & 7,26 & 6,97 & 7,15 & 7,25 \\
$\mathbf{4}$ & 5,65 & 7,4 & 7,66 & 5,64 & 5,52 & 6,98 & 6,62 \\
$\mathbf{5}$ & 5,19 & 6,91 & 6,72 & 6,28 & 5,32 & 6,74 & 6,21 \\
$\mathbf{6}$ & 6,28 & 8,27 & 7,34 & 6,25 & 5,8 & 7,65 & 6,77 \\
$\mathbf{7}$ & 5,14 & 6,49 & 6,77 & 6,06 & 5,64 & 6,76 & 6,29 \\
\hline Média & 5,5 & 6,9 & 7,0 & 5,9 & 5,6 & 6,6 & 6,4 \\
DP & 0,4 & 1,0 & 0,8 & 0,9 & 0,7 & 0,9 & 0,8 \\
\hline
\end{tabular}




\begin{tabular}{|c|c|c|c|c|c|c|c|}
\hline & \multicolumn{2}{|c|}{ Grupo controle } & \multicolumn{2}{|l|}{ ICEVAP } & \multicolumn{2}{|c|}{$\mathrm{mL} . \mathrm{Kg}^{-1}$} & \multirow[b]{2}{*}{ T6 } \\
\hline ANIMAL & T0 & T1 & T2 & T3 & T4 & T5 & \\
\hline 1 & 8,5 & 8,5 & 8,6 & 8,4 & 8,5 & 11 & 11,4 \\
\hline 2 & 8,9 & 10 & 8,8 & 8,8 & 8,9 & 7,8 & 9,28 \\
\hline 3 & 11,4 & 10,3 & 11,5 & 10,5 & 11,1 & 8,9 & 7,94 \\
\hline 4 & 8,4 & 8,3 & 8,5 & 8,5 & 8,2 & 8,6 & 7,8 \\
\hline 5 & 8,5 & 9 & 9,7 & 10,2 & 11 & 10,3 & 10 \\
\hline 6 & 8,6 & 9,7 & 9,9 & 11,2 & 11,5 & 11,5 & 10,5 \\
\hline 7 & 9,7 & 9,5 & 9,4 & 10,4 & 11,4 & 10,7 & 10,6 \\
\hline Média & 9,1 & 9,3 & 9,5 & 9,7 & 10,1 & 9,8 & 9,6 \\
\hline DP & 1,1 & 0,8 & 1,0 & 1,1 & 1,5 & 1,4 & 1,4 \\
\hline
\end{tabular}

Grupo HES ICEVAP

\begin{tabular}{cccccccc}
\hline ANIMAL & T0 & T1 & T2 & T3 & T4 & T5 & T6 \\
\hline $\mathbf{1}$ & 10,5 & 10,9 & 9,8 & 11,3 & 10,7 & 9,4 & 11 \\
$\mathbf{2}$ & 8,8 & 8,9 & 8,5 & 8,8 & 9,1 & 9,6 & 8,9 \\
$\mathbf{3}$ & 8,1 & 8,8 & 8,7 & 9,4 & 10 & 10 & 8,9 \\
$\mathbf{4}$ & 10,1 & 10,7 & 11,3 & 9,9 & 9,7 & 10,3 & 11 \\
$\mathbf{5}$ & 8,1 & 8,4 & 9 & 9 & 8,7 & 9 & 9,2 \\
$\mathbf{6}$ & 10,1 & 10,5 & 11,1 & 11,6 & 11,6 & 13,7 & 13,2 \\
$\mathbf{7}$ & 10,1 & 9,9 & 10,2 & 9,6 & 10 & 9,4 & 9,4 \\
\hline Média & 9,4 & 9,7 & 9,8 & 9,9 & 10,0 & 10,2 & 10,2 \\
DP & 1,0 & 1,0 & 1,1 & 1,1 & 1,0 & 1,6 & 1,6 \\
\hline
\end{tabular}

Grupo Solução salina ICEVAP

\begin{tabular}{cccccccc}
\hline ANIMAL & T0 & T1 & T2 & T3 & T4 & T5 & T6 \\
\hline $\mathbf{1}$ & 9,2 & 9 & 8,6 & 7,9 & 7,9 & 9 & 9,1 \\
$\mathbf{2}$ & 9,9 & 9,3 & 9,6 & 8,9 & 8,2 & 8,4 & 8,5 \\
$\mathbf{3}$ & 10,1 & 8,9 & 10,2 & 10,5 & 10,7 & 11,2 & 10,5 \\
$\mathbf{4}$ & 10,7 & 10,2 & 9,8 & 9,5 & 9,4 & 10,7 & 11,2 \\
$\mathbf{5}$ & 10,2 & 10,1 & 10,2 & 10 & 10 & 12,1 & 12,4 \\
$\mathbf{6}$ & 12,8 & 13,5 & 13,4 & 13,4 & 13 & 14,4 & 15,5 \\
$\mathbf{7}$ & 8,5 & 8,5 & 8 & 8,7 & 9,1 & 9,1 & 8,4 \\
\hline Média & 10,2 & 9,9 & 10,0 & 9,8 & 9,8 & 10,7 & 10,8 \\
DP & 1,4 & 1,7 & 1,7 & 1,8 & 1,7 & 2,1 & 2,5 \\
\hline
\end{tabular}




\begin{tabular}{cccccccc}
\multicolumn{1}{c}{ Grupo controle } & IVSIT & \multicolumn{3}{c}{ $L . \mathbf{m}^{-\mathbf{2}}$} \\
\hline ANIMAL & T0 & T1 & T2 & T3 & T4 & T5 & T6 \\
\hline $\mathbf{1}$ & 822 & 850 & 764 & 790 & 741 & 847 & 872 \\
$\mathbf{2}$ & 932 & 987 & 861 & 814 & 801 & 881 & 969 \\
$\mathbf{3}$ & 973 & 1051 & 953 & 1037 & 941 & 1033 & 829 \\
$\mathbf{4}$ & 925 & 898 & 910 & 834 & 793 & 758 & 719 \\
$\mathbf{5}$ & 787 & 777 & 762 & 762 & 772 & 728 & 761 \\
$\mathbf{6}$ & 791 & 780 & 763 & 775 & 773 & 773 & 757 \\
$\mathbf{7}$ & 823 & 764 & 731 & 715 & 702 & 708 & 678 \\
\hline Média & 864,7 & 872,4 & 820,6 & 818,1 & 789,0 & 818,3 & 797,9 \\
DP & 76,3 & 112,2 & 86,7 & 103,8 & 74,9 & 113,2 & 99,5 \\
\hline
\end{tabular}

\section{Grupo HES} IVSIT

\begin{tabular}{cccccccc}
\hline ANIMAL & T0 & T1 & T2 & T3 & T4 & T5 & T6 \\
\hline $\mathbf{1}$ & 927 & 988 & 1100 & 886 & 847 & 1016 & 872 \\
$\mathbf{2}$ & 835 & 872 & 907 & 805 & 847 & 900 & 848 \\
$\mathbf{3}$ & 724 & 777 & 819 & 722 & 691 & 736 & 681 \\
$\mathbf{4}$ & 871 & 925 & 947 & 805 & 753 & 846 & 798 \\
$\mathbf{5}$ & 819 & 874 & 956 & 864 & 802 & 878 & 789 \\
$\mathbf{6}$ & 850 & 922 & 975 & 858 & 857 & 907 & 880 \\
$\mathbf{7}$ & 802 & 934 & 922 & 794 & 797 & 862 & 827 \\
\hline Média & 832,6 & 898,9 & 946,6 & 819,1 & 799,1 & 877,9 & 813,6 \\
DP & 62,7 & 66,6 & 84,5 & 55,5 & 60,2 & 83,5 & 67,8 \\
\hline
\end{tabular}

Grupo Solução salina

IVSIT

\begin{tabular}{cccccccc}
\hline ANIMAL & T0 & T1 & T2 & T3 & T4 & T5 & T6 \\
\hline $\mathbf{1}$ & 741 & 760 & 779 & 691 & 684 & 725 & 739 \\
$\mathbf{2}$ & 703 & 734 & 713 & 637 & 632 & 687 & 677 \\
$\mathbf{3}$ & 827 & 805 & 798 & 795 & 777 & 818 & 810 \\
$\mathbf{4}$ & 818 & 807 & 823 & 692 & 677 & 813 & 789 \\
$\mathbf{5}$ & 849 & 815 & 774 & 750 & 675 & 845 & 839 \\
$\mathbf{6}$ & 958 & 975 & 799 & 797 & 828 & 907 & 888 \\
$\mathbf{7}$ & 859 & 852 & 742 & 680 & 627 & 721 & 728 \\
\hline Média & 822,1 & 821,1 & 775,4 & 720,3 & 700,0 & 788,0 & 781,4 \\
DP & 83,0 & 77,9 & 37,3 & 61,3 & 74,9 & 79,2 & 72,0 \\
\hline
\end{tabular}


Grupo controle

Freq. resp.

\begin{tabular}{cccccccc}
\hline ANIMAL & T0 & T1 & T2 & T3 & T4 & T5 & T6 \\
\hline $\mathbf{1}$ & 17 & 17 & 17 & 17 & 17 & 18 & 18 \\
$\mathbf{2}$ & 15 & 15 & 15 & 15 & 15 & 15 & 15 \\
$\mathbf{3}$ & 15 & 15 & 15 & 15 & 15 & 15 & 15 \\
$\mathbf{4}$ & 19 & 19 & 19 & 19 & 19 & 19 & 19 \\
$\mathbf{5}$ & 22 & 22 & 22 & 22 & 22 & 22 & 22 \\
$\mathbf{6}$ & 15 & 15 & 15 & 18 & 18 & 18 & 18 \\
$\mathbf{7}$ & 15 & 15 & 15 & 15 & 15 & 15 & 15 \\
\hline Média & 17 & 17 & 17 & 17 & 17 & 17 & 17 \\
DP & 3 & 3 & 3 & 3 & 3 & 3 & 3 \\
\hline
\end{tabular}

Grupo HES

Freq. resp.

\begin{tabular}{cccccccc}
\hline ANIMAL & T0 & T1 & T2 & T3 & T4 & T5 & T6 \\
\hline $\mathbf{1}$ & 15 & 20 & 15 & 15 & 15 & 15 & 15 \\
$\mathbf{2}$ & 18 & 18 & 18 & 18 & 18 & 20 & 18 \\
$\mathbf{3}$ & 17 & 17 & 17 & 19 & 19 & 19 & 19 \\
$\mathbf{4}$ & 18 & 18 & 18 & 18 & 18 & 18 & 18 \\
$\mathbf{5}$ & 15 & 15 & 15 & 15 & 15 & 15 & 15 \\
$\mathbf{6}$ & 23 & 23 & 23 & 23 & 23 & 21 & 21 \\
$\mathbf{7}$ & 20 & 18 & 18 & 18 & 18 & 18 & 20 \\
\hline Média & 18 & 18 & 18 & 18 & 18 & 18 & 18 \\
DP & 3 & 3 & 3 & 3 & 3 & 2 & 2 \\
\hline
\end{tabular}

Grupo Solução salina

Freq. resp.

\begin{tabular}{cccccccc}
\hline ANIMAL & T0 & T1 & T2 & T3 & T4 & T5 & T6 \\
\hline $\mathbf{1}$ & 18 & 18 & 18 & 18 & 18 & 18 & 18 \\
$\mathbf{2}$ & 18 & 18 & 18 & 18 & 18 & 18 & 18 \\
$\mathbf{3}$ & 20 & 20 & 20 & 20 & 20 & 20 & 20 \\
$\mathbf{4}$ & 15 & 15 & 15 & 15 & 15 & 15 & 15 \\
$\mathbf{5}$ & 20 & 20 & 20 & 18 & 18 & 18 & 18 \\
$\mathbf{6}$ & 15 & 15 & 15 & 15 & 15 & 15 & 15 \\
$\mathbf{7}$ & 21 & 21 & 21 & 21 & 21 & 21 & 21 \\
\hline Média & 18 & 18 & 18 & 18 & 18 & 18 & 18 \\
DP & 2 & 2 & 2 & 2 & 2 & 2 & 2 \\
\hline
\end{tabular}




\begin{tabular}{cccccccc} 
& \multicolumn{1}{c}{ Grupo controle } & EtCO2 & \multicolumn{3}{c}{ mmHg } \\
\hline ANIMAL & T0 & T1 & T2 & T3 & T4 & T5 & T6 \\
\hline $\mathbf{1}$ & 42 & 41 & 43 & 43 & 37 & 36 & 36 \\
$\mathbf{2}$ & 46 & 43 & 45 & 50 & 41 & 45 & 42 \\
$\mathbf{3}$ & 37 & 38 & 38 & 39 & 41 & 42 & 43 \\
$\mathbf{4}$ & 39 & 40 & 43 & 44 & 36 & 37 & 33 \\
$\mathbf{5}$ & 43 & 43 & 41 & 40 & 38 & 41 & 40 \\
$\mathbf{6}$ & 43 & 44 & 40 & 42 & 41 & 40 & 39 \\
$\mathbf{7}$ & 43 & 43 & 40 & 45 & 43 & 45 & 46 \\
\hline Média & 42 & 42 & 41 & 43 & 40 & 41 & 40 \\
DP & 3 & 2 & 2 & 4 & 3 & 4 & 4 \\
\hline
\end{tabular}

\section{Grupo HES} EtCO2

\begin{tabular}{cccccccc}
\hline ANIMAL & T0 & T1 & T2 & T3 & T4 & T5 & T6 \\
\hline $\mathbf{1}$ & 40 & 39 & 40 & 38 & 39 & 30 & 42 \\
$\mathbf{2}$ & 39 & 39 & 38 & 37 & 39 & 39 & 38 \\
$\mathbf{3}$ & 41 & 43 & 44 & 41 & 39 & 40 & 40 \\
$\mathbf{4}$ & 44 & 43 & 43 & 46 & 47 & 47 & 48 \\
$\mathbf{5}$ & 39 & 42 & 42 & 41 & 42 & 44 & 40 \\
$\mathbf{6}$ & 41 & 40 & 39 & 36 & 37 & 40 & 37 \\
$\mathbf{7}$ & 37 & 42 & 43 & 46 & 42 & 45 & 40 \\
\hline Média & 40 & 41 & 41 & 41 & 41 & 41 & 41 \\
DP & 2 & 2 & 2 & 4 & 3 & 6 & 4 \\
\hline
\end{tabular}

Grupo Solução salina EtCO2

\begin{tabular}{cccccccc}
\hline ANIMAL & T0 & T1 & T2 & T3 & T4 & T5 & T6 \\
\hline $\mathbf{1}$ & 47 & 43 & 40 & 44 & 44 & 47 & 49 \\
$\mathbf{2}$ & 42 & 42 & 36 & 42 & 42 & 44 & 41 \\
$\mathbf{3}$ & 44 & 42 & 39 & 41 & 41 & 43 & 43 \\
$\mathbf{4}$ & 41 & 44 & 42 & 42 & 44 & 46 & 43 \\
$\mathbf{5}$ & 42 & 45 & 40 & 44 & 43 & 44 & 41 \\
$\mathbf{6}$ & 37 & 37 & 39 & 37 & 37 & 38 & 37 \\
$\mathbf{7}$ & 43 & 39 & 38 & 42 & 40 & 42 & 39 \\
\hline Média & 42 & 42 & 39 & 42 & 42 & 43 & 42 \\
DP & 3 & 3 & 2 & 2 & 3 & 3 & 4 \\
\hline
\end{tabular}




\begin{tabular}{cccccccc}
\multicolumn{2}{l}{ Grupo controle } & $\mathbf{p H}$ \\
\hline ANIMAL & T0 & T1 & T2 & T3 & T4 & T5 & T6 \\
\hline $\mathbf{1}$ & 7,351 & 7,362 & 7,372 & 7,369 & 7,418 & 7,432 & 7,488 \\
$\mathbf{2}$ & 7,397 & 7,4 & 7,393 & 7,394 & 7,407 & 7,318 & 7,35 \\
$\mathbf{3}$ & 7,439 & 7,44 & 7,441 & 7,435 & 7,417 & 7,42 & 7,45 \\
$\mathbf{4}$ & 7,446 & 7,439 & 7,403 & 7,388 & 7,447 & 7,456 & 7,469 \\
$\mathbf{5}$ & 7,389 & 7,437 & 7,437 & 7,433 & 7,454 & 7,471 & 7,479 \\
$\mathbf{6}$ & 7,425 & 7,445 & 7,417 & 7,437 & 7,474 & 7,476 & 7,492 \\
$\mathbf{7}$ & 7,41 & 7,42 & 7,406 & 7,385 & 7,402 & 7,38 & 7,41 \\
\hline Média & 7,41 & 7,42 & 7,41 & 7,41 & 7,43 & 7,42 & 7,45 \\
DP & 0,03 & 0,03 & 0,02 & 0,03 & 0,03 & 0,06 & 0,05 \\
\hline
\end{tabular}

\begin{tabular}{cccccccc} 
Grupo HES & \multicolumn{2}{c}{ pH } & \multicolumn{7}{c}{ T5 } & T6 \\
\hline ANIMAL & T0 & T1 & T2 & T3 & T4 & T5 \\
\hline $\mathbf{1}$ & 7,383 & 7,377 & 7,341 & 7,374 & 7,385 & 7,364 & 7,372 \\
$\mathbf{2}$ & 7,42 & 7,368 & 7,345 & 7,366 & 7,383 & 7,398 & 7,417 \\
$\mathbf{4}$ & 7,312 & 7,3 & 7,259 & 7,327 & 7,36 & 7,381 & 7,409 \\
$\mathbf{7}$ & 7,424 & 7,413 & 7,373 & 7,395 & 7,359 & 7,355 & 7,378 \\
$\mathbf{8}$ & 7,501 & 7,447 & 7,421 & 7,466 & 7,461 & 7,451 & 7,476 \\
$\mathbf{9}$ & 7,461 & 7,458 & 7,45 & 7,469 & 7,462 & 7,425 & 7,448 \\
$\mathbf{1 0}$ & 7,466 & 7,405 & 7,414 & 7,413 & 7,421 & 7,431 & 7,468 \\
\hline Média & 7,42 & 7,40 & 7,37 & 7,40 & 7,40 & 7,40 & 7,42 \\
DP & 0,06 & 0,05 & 0,06 & 0,05 & 0,04 & 0,04 & 0,04 \\
\hline
\end{tabular}

\begin{tabular}{cccccccc}
\multicolumn{2}{l}{ Grupo Solução salina } & \multicolumn{2}{c}{ pH } \\
\hline ANIMAL & T0 & T1 & T2 & T3 & T4 & T5 & T6 \\
\hline $\mathbf{1}$ & 7,337 & 7,272 & 7,244 & 7,294 & 7,286 & 7,279 & 7,275 \\
$\mathbf{2}$ & 7,365 & 7,309 & 7,293 & 7,332 & 7,308 & 7,356 & 7,394 \\
$\mathbf{3}$ & 7,412 & 7,339 & 7,25 & 7,325 & 7,338 & 7,335 & 7,377 \\
$\mathbf{4}$ & 7,419 & 7,363 & 7,271 & 7,358 & 7,366 & 7,348 & 7,409 \\
$\mathbf{5}$ & 7,375 & 7,203 & 7,271 & 7,242 & 7,292 & 7,32 & 7,323 \\
$\mathbf{6}$ & 7,402 & 7,31 & 7,19 & 7,28 & 7,308 & 7,318 & 7,346 \\
$\mathbf{7}$ & 7,417 & 7,413 & 7,348 & 7,411 & 7,421 & 7,42 & 7,445 \\
\hline Média & 7,39 & 7,32 & 7,27 & 7,32 & 7,33 & 7,34 & 7,37 \\
DP & 0,03 & 0,07 & 0,05 & 0,06 & 0,05 & 0,04 & 0,06 \\
\hline
\end{tabular}




\begin{tabular}{cccccccc}
\multicolumn{2}{l}{ Grupo controle } & pCO2 & \multicolumn{2}{c}{$\mathbf{m m g}$} \\
\hline ANIMAL & T0 & T1 & T2 & T3 & T4 & T5 & T6 \\
\hline $\mathbf{1}$ & 45,8 & 44 & 43,5 & 44,7 & 43 & 42,5 & 37,3 \\
$\mathbf{2}$ & 43,1 & 45 & 43,2 & 43,5 & 45,4 & 54,7 & 45 \\
$\mathbf{3}$ & 38,1 & 37,1 & 37,6 & 38,9 & 40,3 & 41,2 & 44 \\
$\mathbf{4}$ & 41,3 & 42,9 & 47,2 & 50,3 & 40,3 & 42,1 & 40,1 \\
$\mathbf{5}$ & 47,9 & 43,6 & 43,9 & 45,9 & 44,9 & 42,6 & 41,3 \\
$\mathbf{6}$ & 45,8 & 44 & 48,1 & 46,9 & 43,2 & 42,5 & 41,3 \\
$\mathbf{7}$ & 46 & 46,2 & 48,9 & 51,7 & 49,2 & 52,1 & 49,7 \\
\hline Média & 44,0 & 43,3 & 44,6 & 46,0 & 43,8 & 45,4 & 42,7 \\
DP & 3,4 & 2,9 & 3,9 & 4,3 & 3,1 & 5,5 & 4,0 \\
\hline
\end{tabular}

\begin{tabular}{|c|c|c|c|c|c|c|c|}
\hline Grupo HE & & $\mathrm{CO2}$ & & & & & \\
\hline ANIMAL & T0 & T1 & T2 & T3 & T4 & T5 & T6 \\
\hline 1 & 46,4 & 42,9 & 45,5 & 44,8 & 45,4 & 46,7 & 50,3 \\
\hline 2 & 40 & 45 & 45 & 44,6 & 43,1 & 42,5 & 43,9 \\
\hline 4 & 44,4 & 47,6 & 51,3 & 49,5 & 47,1 & 43,7 & 44,8 \\
\hline 7 & 46,4 & 46,2 & 50,3 & 49,6 & 55,1 & 55,4 & 53,2 \\
\hline 8 & 39,1 & 43 & 43,2 & 41,5 & 42,8 & 43,9 & 42,4 \\
\hline 9 & 42,1 & 40,5 & 40 & 38,4 & 40 & 44,3 & 41,2 \\
\hline 10 & 40,3 & 45,3 & 42,2 & 46,1 & 44,5 & 44,3 & 41,6 \\
\hline Média & 42,7 & 44,4 & 45,4 & 44,9 & 45,4 & 45,8 & 45,3 \\
\hline DP & 3,1 & 2,4 & 4,1 & 4,1 & 4,8 & 4,4 & 4,6 \\
\hline
\end{tabular}

\begin{tabular}{cccccccc}
\multicolumn{2}{c}{ Grupo Solução salina } & \multicolumn{7}{c}{ pCO2 } \\
\hline ANIMAL & T0 & T1 & T2 & T3 & T4 & T5 & T6 \\
\hline $\mathbf{1}$ & 47,5 & 47,5 & 46,8 & 47,9 & 51,2 & 53,3 & 57,5 \\
$\mathbf{2}$ & 44,9 & 46,4 & 47,2 & 46,6 & 54,1 & 48,5 & 46,2 \\
$\mathbf{3}$ & 45,4 & 48 & 48,5 & 46,2 & 46,7 & 48,1 & 46,8 \\
$\mathbf{4}$ & 43,3 & 40,7 & 43 & 43,4 & 45 & 48,6 & 44,3 \\
$\mathbf{5}$ & 50,8 & 68,8 & 51,2 & 59,6 & 52,4 & 52,1 & 54 \\
$\mathbf{6}$ & 39,9 & 40,5 & 51,4 & 44,4 & 44,6 & 46,3 & 46,8 \\
$\mathbf{7}$ & 43,4 & 42,2 & 45 & 44,1 & 45,1 & 45,9 & 43,5 \\
\hline Média & 45,0 & 47,7 & 47,6 & 47,5 & 48,4 & 49,0 & 48,4 \\
DP & 3,5 & 9,8 & 3,1 & 5,6 & 4,0 & 2,8 & 5,2 \\
\hline
\end{tabular}




\begin{tabular}{cccccccc}
\multicolumn{2}{l}{ Grupo controle } & pO2 & $\mathbf{m m H g}$ & & & \\
\hline ANIMAL & T0 & T1 & T2 & T3 & T4 & T5 & T6 \\
\hline $\mathbf{1}$ & 128,3 & 130,1 & 131,5 & 126,9 & 124,2 & 132 & 113,6 \\
$\mathbf{2}$ & 174,4 & 170,3 & 167,5 & 171,3 & 162,5 & 180,3 & 177,3 \\
$\mathbf{3}$ & 129,4 & 134,4 & 139,4 & 149,8 & 160,1 & 152,1 & 155,1 \\
$\mathbf{4}$ & 142,2 & 146,4 & 126,6 & 149,2 & 149,4 & 143,5 & 149,7 \\
$\mathbf{5}$ & 155,4 & 149,6 & 150,4 & 148,3 & 138,9 & 134,8 & 122 \\
$\mathbf{6}$ & 162,2 & 153,7 & 150,8 & 150 & 159,3 & 162,6 & 171 \\
$\mathbf{7}$ & 144,1 & 143,6 & 116,2 & 108,6 & 104,7 & 102,5 & 100,2 \\
\hline Média & 148,0 & 146,9 & 140,3 & 143,4 & 142,7 & 144,0 & 141,3 \\
DP & 17,0 & 13,2 & 17,3 & 20,0 & 21,7 & 24,8 & 29,6 \\
\hline
\end{tabular}

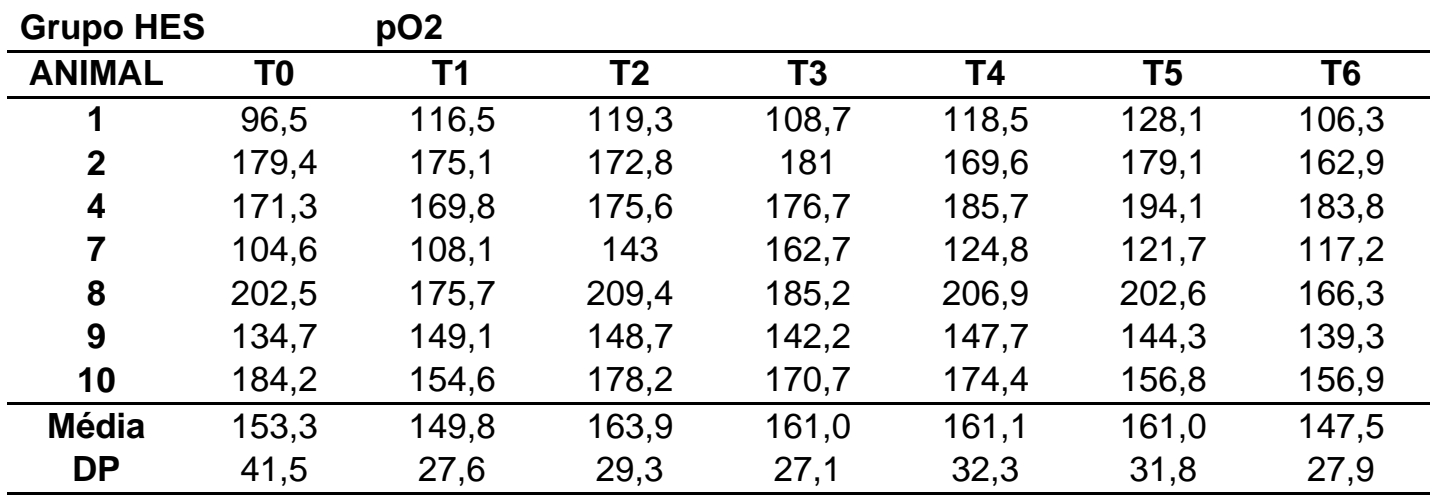

\begin{tabular}{cccccccc}
\multicolumn{2}{c}{ Grupo Solução salina } & \multicolumn{2}{c}{ pO2 } \\
\hline ANIMAL & T0 & T1 & T2 & T3 & T4 & T5 & T6 \\
\hline $\mathbf{1}$ & 130,9 & 136,3 & 163,3 & 138,7 & 138,1 & 137,2 & 142,1 \\
$\mathbf{2}$ & 160,2 & 137,9 & 140 & 145,9 & 156,1 & 133,9 & 128,8 \\
$\mathbf{3}$ & 153,2 & 171,2 & 167,8 & 183,7 & 167,1 & 169,2 & 148,3 \\
$\mathbf{4}$ & 194,3 & 202,4 & 202,4 & 191,6 & 198,3 & 200,4 & 173,2 \\
$\mathbf{5}$ & 98,9 & 76,5 & 90,9 & 88 & 96,1 & 101,6 & 87,6 \\
$\mathbf{6}$ & 77,7 & 75,7 & 67,1 & 86,2 & 93,2 & 75,2 & 70 \\
$\mathbf{7}$ & 195,4 & 195,1 & 172,9 & 189 & 196,3 & 185,1 & 181,5 \\
\hline Média & 144,4 & 142,2 & 143,5 & 146,2 & 149,3 & 143,2 & 133,1 \\
DP & 44,9 & 51,7 & 48,2 & 45,4 & 43,0 & 45,1 & 41,5 \\
\hline
\end{tabular}




\begin{tabular}{cccccccc}
\multicolumn{2}{l}{ Grupo controle } & HCO3 & mmol. $^{-1}$ \\
ANIMAL & T0 & T1 & T2 & T3 & T4 & T5 & T6 \\
\hline $\mathbf{1}$ & 24,3 & 24,5 & 24,5 & 24,9 & 26,7 & 27,1 & 27,9 \\
$\mathbf{2}$ & 26 & 25,7 & 25,1 & 25,1 & 27,2 & 27,2 & 27,5 \\
$\mathbf{3}$ & 25,4 & 24,6 & 25,1 & 25,8 & 26,2 & 25,5 & 26 \\
$\mathbf{4}$ & 27,9 & 28,3 & 28,4 & 29 & 26,9 & 28,7 & 28,2 \\
$\mathbf{5}$ & 27,9 & 28,6 & 28,6 & 29,5 & 30,4 & 30,1 & 29,7 \\
$\mathbf{6}$ & 29,3 & 29,5 & 30 & 30,6 & 30,7 & 30,4 & 30,8 \\
$\mathbf{7}$ & 28,3 & 29 & 29,6 & 29,6 & 29,3 & 30 & 30,3 \\
\hline Média & 27,0 & 28,0 & 27,3 & 27,8 & 28,2 & 28,4 & 29,4 \\
DP & 1,8 & 2,0 & 2,3 & 2,4 & 1,9 & 1,9 & 1,3 \\
\hline
\end{tabular}

\begin{tabular}{cccccccc} 
Grupo HES & \multicolumn{7}{c}{ HCO3 } \\
\hline ANIMAL & T0 & T1 & T2 & T3 & T4 & T5 & T6 \\
\hline $\mathbf{1}$ & 26,6 & 24,3 & 23,7 & 25,5 & 26,2 & 25,7 & 28,1 \\
$\mathbf{2}$ & 25,5 & 24,9 & 23,5 & 24,5 & 24,6 & 25,3 & 27,2 \\
$\mathbf{4}$ & 21,7 & 22,3 & 21,8 & 24,6 & 25,3 & 24,9 & 27,3 \\
$\mathbf{7}$ & 29,8 & 28,7 & 28,2 & 29,3 & 29,7 & 29,6 & 29,9 \\
$\mathbf{8}$ & 30,2 & 29,1 & 27,5 & 29,5 & 30 & 30,1 & 30,8 \\
$\mathbf{9}$ & 29,1 & 27,8 & 27,1 & 27,3 & 27,9 & 28,3 & 27,9 \\
$\mathbf{1 0}$ & 28,5 & 27,5 & 26 & 28,3 & 27,8 & 28,5 & 29,3 \\
\hline Média & 27,3 & 26,4 & 25,4 & 27,0 & 27,4 & 27,5 & 28,6 \\
DP & 3,0 & 2,6 & 2,4 & 2,1 & 2,1 & 2,1 & 1,4 \\
\hline
\end{tabular}

\begin{tabular}{cccccccc}
\multicolumn{2}{c}{ Grupo Solução salina } & \multicolumn{1}{l}{ HCO3 } \\
\hline ANIMAL & T0 & T1 & T2 & T3 & T4 & T5 & T6 \\
\hline $\mathbf{1}$ & 24,2 & 20,7 & 19 & 21,8 & 22,7 & 23,2 & 24,7 \\
$\mathbf{2}$ & 24,8 & 22,3 & 21,7 & 23,4 & 25,2 & 25,9 & 27 \\
$\mathbf{3}$ & 26 & 23,9 & 22,6 & 23,3 & 25,1 & 25,5 & 27,9 \\
$\mathbf{4}$ & 27,5 & 22,4 & 19,2 & 23,6 & 24,8 & 25,7 & 27,1 \\
$\mathbf{5}$ & 28,5 & 25,6 & 22,5 & 24,4 & 24,2 & 26 & 27 \\
$\mathbf{6}$ & 24,4 & 19,7 & 19,2 & 20,2 & 21,5 & 22,8 & 24,6 \\
$\mathbf{7}$ & 26,8 & 25,9 & 23,6 & 26,9 & 28,3 & 28,9 & 29,1 \\
\hline Média & 26,0 & 22,9 & 21,1 & 23,4 & 24,5 & 25,4 & 26,8 \\
DP & 1,7 & 2,3 & 1,9 & 2,1 & 2,1 & 2,0 & 1,6 \\
\hline
\end{tabular}




\begin{tabular}{cccccccc}
\multicolumn{2}{l}{ Grupo controle } & ABE & \multicolumn{7}{c}{ mmol.L $^{-1}$} \\
\hline ANIMAL & T0 & T1 & T2 & T3 & T4 & T5 & T6 \\
\hline $\mathbf{1}$ & $-0,7$ & 1,2 & $-0,3$ & 0,2 & 2,9 & 3,8 & 4,9 \\
$\mathbf{2}$ & 1,3 & 1,1 & 1,2 & 1,5 & 3,3 & 0,4 & 0,7 \\
$\mathbf{3}$ & 2 & 1,3 & 1,7 & 2,4 & 2,8 & 2 & 2,3 \\
$\mathbf{4}$ & 4 & 4,3 & 3,8 & 4,1 & 3,7 & 5,3 & 5,2 \\
$\mathbf{5}$ & 3 & 4,5 & 4,8 & 5,5 & 6,6 & 6,7 & 6,6 \\
$\mathbf{6}$ & 4,7 & 5,4 & 5,2 & 6,3 & 7,3 & 7,1 & 7,6 \\
$\mathbf{7}$ & 3,7 & 4,6 & 4,8 & 4,5 & 4,7 & 4,9 & 5,5 \\
\hline Média & 2,6 & 3,2 & 3,0 & 3,5 & 4,5 & 4,3 & 4,7 \\
DP & 1,9 & 1,9 & 2,2 & 2,2 & 1,8 & 2,4 & 2,4 \\
\hline
\end{tabular}

\begin{tabular}{cccccccc} 
Grupo HES & \multicolumn{2}{c}{ ABE } & \multicolumn{7}{l}{} \\
\hline ANIMAL & T0 & T1 & T2 & T3 & T4 & T5 & T6 \\
\hline $\mathbf{1}$ & 1,9 & $-0,2$ & $-1,5$ & 0,4 & 1,6 & 0,6 & 2,7 \\
$\mathbf{2}$ & 1,5 & 0,2 & $-1,4$ & $-0,1$ & 0,4 & 1,2 & 3,3 \\
$\mathbf{4}$ & -4 & $-3,5$ & $-4,9$ & $-0,8$ & 0,6 & 0,6 & 3,1 \\
$\mathbf{7}$ & 5 & 4,1 & 2,9 & 4,2 & 3,9 & 3,7 & 4,6 \\
$\mathbf{8}$ & 7 & 5 & 3,1 & 5,7 & 6 & 5,7 & 6,9 \\
$\mathbf{9}$ & 5,6 & 4,5 & 3,7 & 4,2 & 4,6 & 4 & 4,2 \\
$\mathbf{1 0}$ & 5 & 3 & 2,2 & 4,1 & 3,9 & 4,5 & 5,8 \\
\hline Média & 3,1 & 1,9 & 0,6 & 2,5 & 3,0 & 2,9 & 4,4 \\
DP & 3,7 & 3,1 & 3,2 & 2,6 & 2,1 & 2,1 & 1,5 \\
\hline
\end{tabular}

\begin{tabular}{cccccccc}
\multicolumn{2}{l}{ Grupo Solução salina } & \multicolumn{7}{c}{ ABE } \\
\hline ANIMAL & T0 & T1 & T2 & T3 & T4 & T5 & T6 \\
\hline $\mathbf{1}$ & $-0,9$ & $-5,3$ & $-7,3$ & $-3,7$ & -3 & $-2,7$ & $-1,6$ \\
$\mathbf{2}$ & $-0,1$ & $-3,3$ & $-4,1$ & $-1,7$ & $-0,3$ & 0,8 & 2,7 \\
$\mathbf{3}$ & 1,4 & $-1,6$ & $-3,4$ & -2 & 0,3 & 0,4 & 3,6 \\
$\mathbf{4}$ & 3,1 & $-2,1$ & $-7,1$ & $-1,3$ & 0 & 0,3 & 2,9 \\
$\mathbf{5}$ & 3,2 & $-3,5$ & $-4,2$ & $-3,4$ & $-2,3$ & $-0,4$ & 0,6 \\
$\mathbf{6}$ & 0,2 & $-5,6$ & $-8,9$ & $-5,8$ & $-4,1$ & $-2,8$ & $-0,7$ \\
$\mathbf{7}$ & 3,1 & 2,2 & $-1,2$ & 2,9 & 4,2 & 4,4 & 5,1 \\
\hline Média & 1,4 & $-2,7$ & $-5,2$ & $-2,1$ & $-0,7$ & 0,0 & 1,8 \\
DP & 1,7 & 2,6 & 2,7 & 2,7 & 2,7 & 2,4 & 2,4 \\
\hline
\end{tabular}




\begin{tabular}{cccccccc}
\multicolumn{6}{l}{ Grupo controle } & SO2 & $\%$ \\
\hline ANIMAL & T0 & T1 & T2 & T3 & T4 & T5 & T6 \\
\hline $\mathbf{1}$ & 98,5 & 99 & 98,6 & 98,4 & 98,4 & 98,6 & 98,5 \\
$\mathbf{2}$ & 99,4 & 99 & 99,2 & 99,2 & 99,1 & 94,6 & 97,2 \\
$\mathbf{3}$ & 98,8 & 98,9 & 99 & 99,1 & 99,2 & 99,1 & 98,7 \\
$\mathbf{4}$ & 99 & 99,1 & 98,5 & 98,9 & 99,1 & 99 & 99,1 \\
$\mathbf{5}$ & 99,1 & 99,1 & 99,1 & 99 & 98,8 & 98,8 & 98,5 \\
$\mathbf{6}$ & 99,3 & 99,2 & 99,1 & 99,1 & 99,2 & 99,2 & 99,3 \\
$\mathbf{7}$ & 99 & 98,9 & 98 & 97,5 & 97,2 & 96,9 & 97,1 \\
\hline Média & 99 & 99 & 99 & 99 & 99 & 98 & 99 \\
DP & 0 & 0 & 0 & 1 & 1 & 2 & 1 \\
\hline
\end{tabular}

\begin{tabular}{|c|c|c|c|c|c|c|c|}
\hline Grupo HE & & 02 & & & & & \\
\hline ANIMAL & T0 & T1 & T2 & T3 & T4 & T5 & T6 \\
\hline 1 & 96,7 & 98,1 & 98,1 & 97,9 & 98,2 & 98,5 & 97,5 \\
\hline 2 & 99,4 & 99,3 & 99,2 & 99,3 & 99,2 & 99,4 & 99,2 \\
\hline 4 & 99,2 & 99,1 & 99,1 & 99,2 & 99,4 & 99,5 & 99,4 \\
\hline 7 & 97,9 & 97,8 & 98,8 & 99,2 & 98,2 & 98 & 97,9 \\
\hline 8 & 99,6 & 99,4 & 99,6 & 99,5 & 99,6 & 99,6 & 99,4 \\
\hline 9 & 98,8 & 99,1 & 99,1 & 99 & 99,1 & 99 & 99 \\
\hline 10 & 99,5 & 99,1 & 99,4 & 99,3 & 99,3 & 99,2 & 99,2 \\
\hline Média & 99 & 99 & 99 & 99 & 99 & 99 & 99 \\
\hline DP & 1 & 1 & 0 & 1 & 1 & 1 & 1 \\
\hline
\end{tabular}

\begin{tabular}{cccccccc}
\multicolumn{2}{l}{ Grupo Solução salina } & \multicolumn{2}{c}{ SO2 } \\
\hline ANIMAL & T0 & T1 & T2 & T3 & T4 & T5 & T6 \\
\hline $\mathbf{1}$ & 98,3 & 98,3 & 98,9 & 98,4 & 98,2 & 98,1 & 98,2 \\
$\mathbf{2}$ & 99,1 & 98,6 & 98,6 & 98,8 & 98,7 & 98,5 & 98,4 \\
$\mathbf{3}$ & 99,4 & 99,2 & 99,1 & 99,3 & 99,2 & 99,2 & 98,9 \\
$\mathbf{4}$ & 99,5 & 99,5 & 99,5 & 99,4 & 99,5 & 99,5 & 99,3 \\
$\mathbf{5}$ & 96,8 & 90,3 & 95,3 & 94,3 & 96,1 & 97,1 & 95,4 \\
$\mathbf{6}$ & 95,3 & 93,3 & 88,1 & 95 & 96 & 93,1 & 91,9 \\
$\mathbf{7}$ & 99,5 & 99,5 & 99,2 & 99,4 & 99,5 & 99,4 & 99,4 \\
\hline Média & 98 & 97 & 97 & 98 & 98 & 98 & 97 \\
DP & 2 & 4 & 4 & 2 & 2 & 2 & 3 \\
\hline
\end{tabular}




\begin{tabular}{cccccccc}
\multicolumn{2}{l}{ Grupo controle } & $\mathbf{K}$ & mmol. $^{-\mathbf{1}}$ & & & \\
\hline ANIMAL & T0 & T1 & T2 & T3 & T4 & T5 & T6 \\
\hline $\mathbf{1}$ & 3,3 & 3,5 & 3,8 & 4,1 & 4,4 & 4,6 & 4,8 \\
$\mathbf{2}$ & 4,1 & 4,2 & 4,5 & 4,4 & 4,4 & 4,4 & 4,3 \\
$\mathbf{3}$ & 4 & 3,9 & 4 & 4,2 & 4,3 & 4,3 & 4,4 \\
$\mathbf{4}$ & 3,8 & 3,8 & 3,9 & 3,8 & 3,5 & 3,9 & 3,9 \\
$\mathbf{5}$ & 3,7 & 4,1 & 4,1 & 4,2 & 4,4 & 4 & 4 \\
$\mathbf{6}$ & 3,7 & 3,8 & 3,8 & 4,2 & 4 & 4 & 4,2 \\
$\mathbf{7}$ & 3,8 & 4,1 & 4,2 & 4,5 & 4,4 & 4,3 & 4,2 \\
\hline Média & 3,8 & 3,9 & 4,0 & 4,2 & 4,2 & 4,2 & 4,2 \\
DP & 0,3 & 0,2 & 0,3 & 0,2 & 0,3 & 0,3 & 0,3 \\
\hline
\end{tabular}

\begin{tabular}{|c|c|c|c|c|c|c|c|}
\hline Grupo HES & & & & & & & \\
\hline ANIMAL & T0 & T1 & T2 & T3 & T4 & T5 & T6 \\
\hline 1 & 3,8 & 3,6 & 3,7 & 4,3 & 4,5 & 4,3 & 4,4 \\
\hline 2 & 3,8 & 3,8 & 3,8 & 3,8 & 3,9 & 3,7 & 4,2 \\
\hline 4 & 3,2 & 3,4 & 3,3 & 3,7 & 3,8 & 3,3 & 3,6 \\
\hline 7 & 3,5 & 3,4 & 3,6 & 3,9 & 4 & 4 & 4,2 \\
\hline 8 & 3,5 & 3,6 & 3,6 & 4,1 & 4 & 3,8 & 4 \\
\hline 9 & 4,2 & 3,7 & 3,7 & 4,2 & 4,4 & 4,3 & 4,4 \\
\hline 10 & 3,8 & 3,5 & 3,5 & 3,7 & 3,6 & 3,6 & 3,7 \\
\hline Média & 3,7 & 3,6 & 3,6 & 4,0 & 4,0 & 3,9 & 4,1 \\
\hline DP & 0,3 & 0,1 & 0,2 & 0,2 & 0,3 & 0,4 & 0,3 \\
\hline
\end{tabular}

\begin{tabular}{cccccccc}
\multicolumn{2}{l}{ Grupo Solução salina } & \multicolumn{2}{c}{$\mathbf{K}$} & \multicolumn{2}{l}{} \\
\hline ANIMAL & T0 & T1 & T2 & T3 & T4 & T5 & T6 \\
\hline $\mathbf{1}$ & 4,3 & 3,6 & 3,7 & 4 & 4,3 & 4,2 & 4,2 \\
$\mathbf{2}$ & 4 & 3,6 & 3,9 & 4 & 4,3 & 4 & 4,2 \\
$\mathbf{3}$ & 3,6 & 3,4 & 3,5 & 3,6 & 3,9 & 3,7 & 4,2 \\
$\mathbf{4}$ & 3,4 & 2,9 & 3 & 3,6 & 3,9 & 3,7 & 3,8 \\
$\mathbf{5}$ & 3,6 & 3,5 & 3,4 & 4 & 4,2 & 4,1 & 4 \\
$\mathbf{6}$ & 3,5 & 2,9 & 3,2 & 3,5 & 4 & 3,9 & 4 \\
$\mathbf{7}$ & 3,74 & 3,2 & 3,1 & 3,5 & 3,6 & 3,3 & 3,5 \\
\hline Média & 3,7 & 3,3 & 3,4 & 3,7 & 4,0 & 3,8 & 4,0 \\
DP & 0,3 & 0,3 & 0,3 & 0,2 & 0,3 & 0,3 & 0,3 \\
\hline
\end{tabular}




\begin{tabular}{cccccccc}
\multicolumn{2}{l}{ Grupo controle } & $\mathbf{N a}$ & mmol. $^{-\mathbf{1}}$ & & & \\
\hline ANIMAL & T0 & T1 & T2 & T3 & T4 & T5 & T6 \\
\hline $\mathbf{1}$ & 140 & 140 & 141 & 141 & 137 & 136 & 134 \\
$\mathbf{2}$ & 142 & 143 & 147 & 146 & 138 & 146 & 145 \\
$\mathbf{3}$ & 139 & 140 & 138 & 137 & 137 & 137 & 136 \\
$\mathbf{4}$ & 135 & 133 & 134 & 134 & 136 & 134 & 134 \\
$\mathbf{5}$ & 140 & 139 & 138 & 137 & 137 & 137 & 138 \\
$\mathbf{6}$ & 137 & 136 & 135 & 135 & 134 & 134 & 133 \\
$\mathbf{7}$ & 137 & 137 & 136 & 136 & 136 & 135 & 137 \\
\hline Média & 138,6 & 137,0 & 138,4 & 138,0 & 136,4 & 137,0 & 135,2 \\
DP & 2,4 & 2,7 & 4,4 & 4,2 & 1,3 & 4,2 & 2,2 \\
\hline
\end{tabular}

\begin{tabular}{|c|c|c|c|c|c|c|c|}
\hline Grupo HE & & la & & & & & \\
\hline ANIMAL & TO & T1 & T2 & T3 & T4 & T5 & T6 \\
\hline 1 & 142 & 142 & 143 & 142 & 142 & 144 & 140 \\
\hline 2 & 133 & 140 & 140 & 140 & 140 & 141 & 141 \\
\hline 4 & 140 & 138 & 138 & 137 & 137 & 137 & 138 \\
\hline 7 & 138 & 137 & 136 & 138 & 137 & 138 & 137 \\
\hline 8 & 138 & 138 & 137 & 139 & 139 & 139 & 139 \\
\hline 9 & 138 & 137 & 138 & 137 & 137 & 138 & 137 \\
\hline 10 & 139 & 137 & 137 & 137 & 138 & 139 & 138 \\
\hline Média & 138,3 & 138,4 & 138,4 & 138,6 & 138,6 & 139,4 & 138,6 \\
\hline DP & 2,8 & 1,9 & 2,4 & 1,9 & 1,9 & 2,4 & 1,5 \\
\hline
\end{tabular}

\begin{tabular}{cccccccc}
\multicolumn{2}{l}{ Grupo Solução salina } & \multicolumn{2}{c}{ Na } \\
\hline Porco & T0 & T1 & T2 & T3 & T4 & T5 & T6 \\
\hline $\mathbf{1}$ & 138 & 140 & 140 & 135 & 136 & 139 & 137 \\
$\mathbf{2}$ & 144 & 146 & 145 & 140 & 139 & 140 & 139 \\
$\mathbf{3}$ & 139 & 139 & 139 & 139 & 138 & 139 & 136 \\
$\mathbf{4}$ & 137 & 138 & 138 & 137 & 137 & 137 & 136 \\
$\mathbf{5}$ & 139 & 141 & 141 & 138 & 139 & 140 & 138 \\
$\mathbf{6}$ & 139 & 140 & 140 & 140 & 138 & 138 & 138 \\
$\mathbf{7}$ & 138,5 & 142 & 143 & 142 & 141 & 141 & 141 \\
\hline Média & 139,2 & 140,9 & 140,9 & 138,7 & 138,3 & 139,1 & 137,9 \\
DP & 2,2 & 2,6 & 2,4 & 2,3 & 1,6 & 1,3 & 1,8 \\
\hline
\end{tabular}




\begin{tabular}{cccccccc}
\multicolumn{2}{l}{ Grupo controle } & LACTATO & \multicolumn{7}{c}{ mmol. $^{-1}$} \\
\hline ANIMAL & T0 & T1 & T2 & T3 & T4 & T5 & T6 \\
\hline $\mathbf{1}$ & 1,1 & 1,2 & 1,1 & 1,3 & 1,3 & 1,1 & 1 \\
$\mathbf{2}$ & 0,9 & 0,8 & 0,6 & 0,5 & 0,5 & 0,4 & 0,6 \\
$\mathbf{3}$ & 0,7 & 0,7 & 0,7 & 0,9 & 0,6 & 0,6 & 0,5 \\
$\mathbf{4}$ & 1,3 & 1,3 & 1,3 & 1 & 0,8 & 0,8 & 0,7 \\
$\mathbf{5}$ & 0,8 & 0,9 & 1 & 1,1 & 0,8 & 0,6 & 0,5 \\
$\mathbf{6}$ & 1,4 & 1,5 & 1,4 & 1,5 & 1 & 1,3 & 1,2 \\
$\mathbf{7}$ & 1,7 & 1,6 & 1,6 & 1,6 & 1,2 & 1 & 0,7 \\
\hline Média & 1,13 & 1,14 & 1,10 & 1,13 & 0,89 & 0,83 & 0,74 \\
DP & 0,36 & 0,35 & 0,37 & 0,38 & 0,30 & 0,32 & 0,26 \\
\hline
\end{tabular}

\begin{tabular}{cccccccc} 
Grupo HES & \multicolumn{7}{c}{ LACTATO } \\
\hline ANIMAL & T0 & T1 & T2 & T3 & T4 & T5 & T6 \\
\hline $\mathbf{1}$ & 1,6 & 1,6 & 1,6 & 1,5 & 1 & 1 & 1,2 \\
$\mathbf{2}$ & 1,5 & 1,2 & 1,2 & 1 & 0,9 & 1 & 0,9 \\
$\mathbf{4}$ & 1,9 & 1,8 & 1,6 & 1,7 & 1,7 & 1,7 & 1,2 \\
$\mathbf{7}$ & 1,4 & 1,4 & 1,3 & 1 & 1,3 & 1,3 & 1,2 \\
$\mathbf{8}$ & 1,8 & 2,1 & 1,7 & 1,8 & 1,4 & 1,5 & 1,2 \\
$\mathbf{9}$ & 2 & 1,4 & 1,2 & 1,2 & 1,4 & 1,4 & 1 \\
$\mathbf{1 0}$ & 1,5 & 1,2 & 1,1 & 0,9 & 0,8 & 0,8 & 0,9 \\
\hline Média & 1,67 & 1,53 & 1,39 & 1,30 & 1,21 & 1,24 & 1,09 \\
DP & 0,23 & 0,33 & 0,24 & 0,37 & 0,32 & 0,32 & 0,15 \\
\hline
\end{tabular}

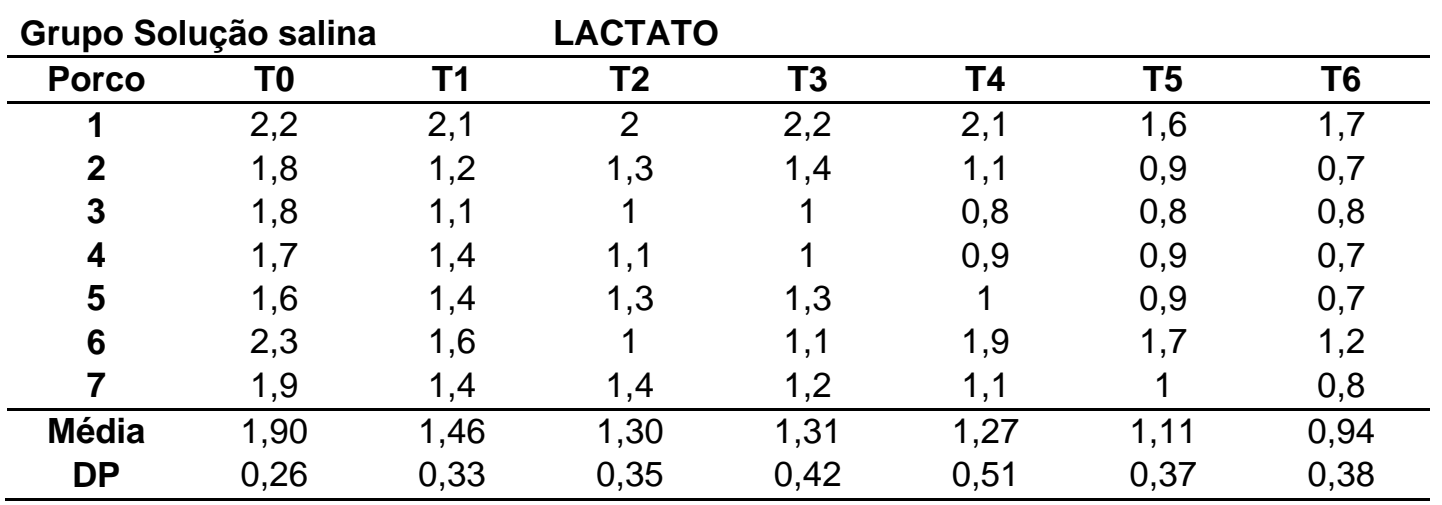




\begin{tabular}{cccccccc}
\multicolumn{2}{l}{ Grupo controle } & \multicolumn{2}{l}{ HEMOGLOBINA } & g.dL $^{-\mathbf{1}}$ & & \\
\hline ANIMAL & T0 & T1 & T2 & T3 & T4 & T5 & T6 \\
\hline $\mathbf{1}$ & 10 & 9,8 & 9,3 & 10 & 10,9 & 10,9 & 11 \\
$\mathbf{2}$ & 8,7 & 8,6 & 8,5 & 8,5 & 9,3 & 11,3 & 11,4 \\
$\mathbf{3}$ & 8,6 & 8,6 & 9,3 & 8,6 & 9,3 & 9,7 & 9,8 \\
$\mathbf{4}$ & 8 & 8,6 & 9 & 8 & 7,3 & 9 & 10 \\
$\mathbf{5}$ & 8,3 & 9 & 8,6 & 8,6 & 9 & 9,3 & 9,3 \\
$\mathbf{6}$ & 10,6 & 10,6 & 11,3 & 11,3 & 11,3 & 11,6 & 11,6 \\
$\mathbf{7}$ & 9 & 9,3 & 9,6 & 9,6 & 9,6 & 10 & 10 \\
\hline Média & 9,0 & 9,2 & 9,4 & 9,2 & 9,5 & 10,3 & 10,4 \\
DP & 0,9 & 0,8 & 0,9 & 1,1 & 1,3 & 1,0 & 0,9 \\
\hline
\end{tabular}

\begin{tabular}{cccccccc} 
Grupo HES & \multicolumn{7}{c}{ HEMOGLOBINA } \\
\hline ANIMAL & T0 & T1 & T2 & T3 & T4 & T5 & T6 \\
\hline $\mathbf{1}$ & 9 & 6 & 5 & 4,6 & 6,3 & 7 & 8 \\
$\mathbf{2}$ & 9 & 6,3 & 5 & 5,6 & 5,6 & 7,3 & 8 \\
$\mathbf{4}$ & 8,3 & 6,3 & 4,3 & 5,3 & 7 & 7 & 8,3 \\
$\mathbf{7}$ & 9,3 & 6,3 & 5 & 7 & 7,6 & 7,6 & 9 \\
$\mathbf{8}$ & 9,3 & 6 & 4,6 & 5,6 & 6 & 7,3 & 8,3 \\
$\mathbf{9}$ & 9,3 & 6,6 & 5 & 6 & 7,3 & 8,3 & 8,6 \\
$\mathbf{1 0}$ & 9,3 & 6 & 5 & 5,3 & 5,3 & 6,6 & 7,6 \\
\hline Média & 9,1 & 6,2 & 4,8 & 5,6 & 6,4 & 7,3 & 8,3 \\
DP & 0,4 & 0,2 & 0,3 & 0,7 & 0,9 & 0,5 & 0,5 \\
\hline
\end{tabular}

\begin{tabular}{cccccccc}
\multicolumn{2}{c}{ Grupo Solução salina } & \multicolumn{7}{c}{ HEMOGLOBINA } \\
\hline Porco & T0 & T1 & T2 & T3 & T4 & T5 & T6 \\
\hline $\mathbf{1}$ & 8,3 & 7 & 5 & 6 & 6 & 6 & 7 \\
$\mathbf{2}$ & 8,3 & 6 & 5,3 & 8 & 10 & 8,3 & 8,6 \\
$\mathbf{3}$ & 8,8 & 6,3 & 5 & 6 & 7 & 7,6 & 9 \\
$\mathbf{4}$ & 9,3 & 6,3 & 4,6 & 5,6 & 5,6 & 6,6 & 7,3 \\
$\mathbf{5}$ & 9,3 & 7 & 5 & 7,3 & 7,3 & 8 & 8,3 \\
$\mathbf{6}$ & 10 & 6 & 5 & 7 & 7,3 & 9 & 9,3 \\
$\mathbf{7}$ & 8 & 6,3 & 5 & 6,3 & 6,6 & 7,3 & 7,6 \\
\hline Média & 8,9 & 6,4 & 5,0 & 6,6 & 7,1 & 7,5 & 8,2 \\
DP & 0,7 & 0,4 & 0,2 & 0,9 & 1,4 & 1,0 & 0,9 \\
\hline
\end{tabular}


Grupo controle HEMATÓCRITO \%

\begin{tabular}{cccccccc}
\hline ANIMAL & T0 & T1 & T2 & T3 & T4 & T5 & T6 \\
\hline $\mathbf{1}$ & 30 & 29 & 29 & 30 & 32 & 32 & 33 \\
$\mathbf{2}$ & 26 & 26 & 26 & 25 & 25 & 28 & 34 \\
$\mathbf{3}$ & 26 & 26 & 28 & 26 & 28 & 29 & 30 \\
$\mathbf{4}$ & 24 & 26 & 27 & 24 & 22 & 27 & 30 \\
$\mathbf{5}$ & 25 & 27 & 26 & 26 & 27 & 28 & 28 \\
$\mathbf{6}$ & 32 & 32 & 34 & 34 & 34 & 35 & 35 \\
$\mathbf{7}$ & 27 & 28 & 29 & 29 & 29 & 30 & 30 \\
\hline Média & 27 & 28 & 28 & 28 & 28 & 30 & 32 \\
DP & 3 & 2 & 3 & 3 & 4 & 3 & 3 \\
\hline
\end{tabular}

\begin{tabular}{cccccccc} 
Grupo HES & \multicolumn{7}{c}{ HEMATÓCRITO } \\
\hline ANIMAL & T0 & T1 & T2 & T3 & T4 & T5 & T6 \\
\hline $\mathbf{1}$ & 27 & 18 & 15 & 14 & 19 & 20 & 24 \\
$\mathbf{2}$ & 26 & 19 & 15 & 17 & 17 & 22 & 24 \\
$\mathbf{4}$ & 25 & 19 & 13 & 16 & 20 & 21 & 25 \\
$\mathbf{7}$ & 28 & 19 & 15 & 21 & 23 & 23 & 28 \\
$\mathbf{8}$ & 28 & 18 & 14 & 17 & 18 & 22 & 25 \\
$\mathbf{9}$ & 28 & 20 & 15 & 18 & 22 & 25 & 26 \\
$\mathbf{1 0}$ & 28 & 18 & 15 & 16 & 16 & 20 & 23 \\
\hline Média & 27 & 19 & 15 & 17 & 19 & 22 & 25 \\
DP & 1 & 1 & 1 & 2 & 3 & 2 & 2 \\
\hline
\end{tabular}

\begin{tabular}{cccccccc}
\multicolumn{7}{c}{ Grupo Solução salina } & \multicolumn{7}{c}{ HEMATÓCRITO } \\
\hline ANIMAL & T0 & T1 & T2 & T3 & T4 & T5 & T6 \\
\hline $\mathbf{1}$ & 25 & 20 & 15 & 18 & 18 & 18 & 20 \\
$\mathbf{2}$ & 25 & 18 & 16 & 23 & 29 & 25 & 26 \\
$\mathbf{3}$ & 27 & 19 & 15 & 18 & 21 & 23 & 27 \\
$\mathbf{4}$ & 28 & 19 & 14 & 17 & 17 & 20 & 22 \\
$\mathbf{5}$ & 28 & 21 & 15 & 22 & 22 & 24 & 25 \\
$\mathbf{6}$ & 30 & 18 & 15 & 21 & 22 & 27 & 28 \\
$\mathbf{7}$ & 24 & 19 & 15 & 19 & 20 & 22 & 23 \\
\hline Média & 27 & 19 & 15 & 20 & 21 & 23 & 24 \\
DP & 2 & 1 & 1 & 2 & 4 & 3 & 3 \\
\hline
\end{tabular}




\begin{tabular}{cccccccc}
\multicolumn{2}{l}{ Grupo controle } & \multicolumn{2}{l}{ SVO2 } & VENOSA MISTA & \% & \\
\hline ANIMAL & T0 & T1 & T2 & T3 & T4 & T5 & T6 \\
\hline $\mathbf{1}$ & 79,8 & 78 & 75,1 & 75 & 74,2 & 71,8 & 70,3 \\
$\mathbf{2}$ & 70,8 & 69 & 67,1 & 65,6 & 71,2 & 72,7 & 73,7 \\
$\mathbf{3}$ & 81,3 & 81 & 80,9 & 81,3 & 79,4 & 78,6 & 78,7 \\
$\mathbf{4}$ & 75,6 & 79,3 & 77,3 & 77,4 & 76,6 & 78 & 82,6 \\
$\mathbf{5}$ & 76,9 & 76,3 & 72,6 & 71,5 & 72,3 & 76,1 & 75 \\
$\mathbf{6}$ & 84 & 82,2 & 82,1 & 81,6 & 79,8 & 81,9 & 81,5 \\
$\mathbf{7}$ & 82,7 & 78,4 & 78 & 76,2 & 78,3 & 78,7 & 76,2 \\
\hline Média & 78,7 & 77,7 & 76,2 & 75,5 & 76,0 & 76,8 & 76,9 \\
DP & 4,6 & 4,3 & 5,1 & 5,6 & 3,5 & 3,6 & 4,4 \\
\hline
\end{tabular}

\begin{tabular}{cccccccc} 
Grupo HES & \multicolumn{7}{c}{ SVO2 VENOSA MISTA } \\
\hline ANIMAL & T0 & T1 & T2 & T3 & T4 & T5 & T6 \\
\hline $\mathbf{1}$ & 80,5 & 77,2 & 77,2 & 76,8 & 78 & 76,9 & 80,8 \\
$\mathbf{2}$ & 79,9 & 78 & 70,3 & 69,1 & 69,1 & 76,7 & 77,1 \\
$\mathbf{4}$ & 81,2 & 79,6 & 71,9 & 72,3 & 74,8 & 77,2 & 80,1 \\
$\mathbf{7}$ & 80,1 & 75,1 & 74,2 & 74,8 & 77,3 & 78,8 & 79,5 \\
$\mathbf{8}$ & 81,3 & 84 & 82,7 & 83,2 & 80,9 & 84,6 & 83,1 \\
$\mathbf{9}$ & 74,4 & 72,3 & 72,7 & 71,6 & 72,1 & 78,1 & 75,5 \\
$\mathbf{1 0}$ & 81,8 & 73,9 & 69,4 & 69,3 & 70 & 78 & 80,6 \\
\hline Média & 79,9 & 77,2 & 74,1 & 73,9 & 74,6 & 78,6 & 79,5 \\
DP & 2,5 & 3,9 & 4,6 & 5,0 & 4,4 & 2,7 & 2,5 \\
\hline
\end{tabular}

Grupo Solução salina

SVO2 VENOSA MISTA

\begin{tabular}{cccccccc}
\hline ANIMAL & T0 & T1 & T2 & T3 & T4 & T5 & T6 \\
\hline $\mathbf{1}$ & 77,1 & 73,5 & 70,9 & 63,9 & 60,8 & 67,8 & 69,8 \\
$\mathbf{2}$ & 80,4 & 75,2 & 69,9 & 65,6 & 69 & 72,3 & 71,8 \\
$\mathbf{3}$ & 79,6 & 80,8 & 78,7 & 81,8 & 78,1 & 84,5 & 83,5 \\
$\mathbf{4}$ & 88 & 86,6 & 82,6 & 76,9 & 74,9 & 83 & 83,7 \\
$\mathbf{5}$ & 76,5 & 68,7 & 67,5 & 66,3 & 67,5 & 79,3 & 74,8 \\
$\mathbf{6}$ & 79,2 & 75,5 & 67 & 71,4 & 72 & 77,5 & 75,8 \\
$\mathbf{7}$ & 71 & 76,8 & 76,3 & 73,5 & 76,1 & 81,7 & 79,7 \\
\hline Média & 78,8 & 76,7 & 73,3 & 71,3 & 71,2 & 78,0 & 77,0 \\
DP & 5,1 & 5,7 & 6,0 & 6,6 & 6,0 & 6,0 & 5,5 \\
\hline
\end{tabular}




\begin{tabular}{cccccccc}
\multicolumn{2}{l}{ Grupo controle } & PvO2 & mmHg & & & \\
\hline ANIMAL & T0 & T1 & T2 & T3 & T4 & T5 & T6 \\
\hline $\mathbf{1}$ & 49,9 & 45 & 44,2 & 45,4 & 45 & 43,9 & 35,8 \\
$\mathbf{2}$ & 43,4 & 42,4 & 41,6 & 41,7 & 44,7 & 53,1 & 54,3 \\
$\mathbf{3}$ & 45,9 & 45,7 & 46,2 & 47,3 & 44,1 & 47,9 & 49 \\
$\mathbf{4}$ & 41,7 & 46,2 & 46,8 & 48,2 & 45,2 & 45,2 & 50,5 \\
$\mathbf{5}$ & 48,1 & 44,7 & 43,8 & 43,6 & 43,4 & 45 & 43,5 \\
$\mathbf{6}$ & 50,3 & 49,5 & 50 & 50,8 & 47,7 & 49,7 & 48,2 \\
$\mathbf{7}$ & 51,1 & 48,2 & 48,3 & 47,7 & 49,8 & 50,5 & 47,4 \\
\hline Média & 47,2 & 46,9 & 45,8 & 46,4 & 45,7 & 47,9 & 45,1 \\
DP & 3,6 & 2,0 & 2,9 & 3,1 & 2,3 & 3,4 & 5,8 \\
\hline
\end{tabular}

\begin{tabular}{|c|c|c|c|c|c|c|c|}
\hline Grupo HE & & vO2 & & & & & \\
\hline ANIMAL & T0 & T1 & T2 & T3 & T4 & T5 & T6 \\
\hline 1 & 50,6 & 47,1 & 48 & 47,1 & 47,2 & 46,7 & 50,5 \\
\hline 2 & 50 & 48 & 43,7 & 43 & 42,8 & 47,2 & 47,3 \\
\hline 4 & 53,3 & 54,2 & 48,3 & 47,7 & 48,6 & 49,2 & 49,4 \\
\hline 7 & 46,8 & 43,2 & 44,8 & 46 & 49,2 & 50,8 & 51,9 \\
\hline 8 & 43,9 & 48,8 & 47,5 & 47,9 & 44,7 & 50,2 & 46,7 \\
\hline 9 & 42,8 & 41,4 & 41,7 & 39 & 40 & 44,7 & 41,5 \\
\hline 10 & 47 & 43 & 40,8 & 42,1 & 42,1 & 46,5 & 46,4 \\
\hline Média & 47,8 & 46,5 & 45,0 & 44,7 & 44,9 & 47,9 & 47,7 \\
\hline DP & 3,8 & 4,4 & 3,1 & 3,4 & 3,5 & 2,2 & 3,4 \\
\hline
\end{tabular}

\begin{tabular}{cccccccc}
\multicolumn{2}{c}{ Grupo Solução salina } & \multicolumn{7}{c}{ PvO2 } & & & \\
\hline ANIMAL & T0 & T1 & T2 & T3 & T4 & T5 & T6 \\
\hline $\mathbf{1}$ & 52,2 & 51,5 & 51,3 & 45,3 & 44,2 & 48,6 & 51,1 \\
$\mathbf{2}$ & 50,4 & 48,3 & 46,1 & 42,8 & 45,7 & 46,1 & 44,8 \\
$\mathbf{3}$ & 50,2 & 55 & 52,4 & 55,1 & 51,3 & 57,9 & 55,6 \\
$\mathbf{4}$ & 55,8 & 59,2 & 53,3 & 46,8 & 46,1 & 54 & 52,5 \\
$\mathbf{5}$ & 47,7 & 47,8 & 44,6 & 43,7 & 43,8 & 50,2 & 46,3 \\
$\mathbf{6}$ & 45,3 & 44 & 43,7 & 43,7 & 44,3 & 48,5 & 45,5 \\
$\mathbf{7}$ & 44,2 & 47,3 & 48,1 & 44 & 46,3 & 49,3 & 46,4 \\
\hline Média & 49,4 & 50,4 & 48,5 & 45,9 & 46,0 & 50,7 & 48,9 \\
DP & 4,0 & 5,2 & 3,9 & 4,3 & 2,6 & 4,0 & 4,2 \\
\hline
\end{tabular}




\begin{tabular}{cccccccc}
\multicolumn{2}{l}{ Grupo controle } & CaO2 & mL.dL $^{-1}$ & & & \\
\hline ANIMAL & T0 & T1 & T2 & T3 & T4 & T5 & T6 \\
\hline $\mathbf{1}$ & 13,991 & 13,070 & 13,062 & 13,973 & 15,186 & 15,241 & 15,304 \\
$\mathbf{2}$ & 12,475 & 12,270 & 12,155 & 12,167 & 13,222 & 15,001 & 15,030 \\
$\mathbf{3}$ & 12,127 & 12,154 & 13,138 & 12,226 & 13,228 & 13,737 & 13,800 \\
$\mathbf{4}$ & 11,370 & 12,215 & 12,626 & 11,381 & 10,446 & 12,741 & 14,140 \\
$\mathbf{5}$ & 11,833 & 12,772 & 12,227 & 12,209 & 12,702 & 13,098 & 13,020 \\
$\mathbf{6}$ & 15,028 & 14,987 & 15,921 & 15,919 & 15,963 & 16,384 & 16,426 \\
$\mathbf{7}$ & 12,743 & 13,138 & 13,343 & 13,253 & 13,202 & 13,690 & 13,710 \\
\hline Média & 12,80 & 12,94 & 13,21 & 13,02 & 13,42 & 14,27 & 14,49 \\
DP & 1,29 & 0,99 & 1,28 & 1,53 & 1,78 & 1,31 & 1,16 \\
\hline
\end{tabular}

\begin{tabular}{|c|c|c|c|c|c|c|c|}
\hline Grupo HE & & $\mathrm{aO2}$ & & & & & \\
\hline ANIMAL & T0 & T1 & T2 & T3 & T4 & T5 & T6 \\
\hline 1 & 12,309 & 8,484 & 7,139 & 6,552 & 8,905 & 9,912 & 11,094 \\
\hline 2 & 12,902 & 9,176 & 7,380 & 8,235 & 8,192 & 10,569 & 11,457 \\
\hline 4 & 11,893 & 9,142 & 6,425 & 7,803 & 10,178 & 10,213 & 11,955 \\
\hline 7 & 12,889 & 8,838 & 7,261 & 10,087 & 10,686 & 10,656 & 12,523 \\
\hline 8 & 13,410 & 8,775 & 6,972 & 8,263 & 8,888 & 10,662 & 11,901 \\
\hline 9 & 13,098 & 9,488 & 7,299 & 8,638 & 10,441 & 11,787 & 12,181 \\
\hline 10 & 13,341 & 8,685 & 7,411 & 7,792 & 7,803 & 9,521 & 10,890 \\
\hline Média & 12,83 & 8,94 & 7,13 & 8,20 & 9,30 & 10,47 & 11,71 \\
\hline DP & 0,55 & 0,34 & 0,34 & 1,06 & 1,14 & 0,72 & 0,59 \\
\hline
\end{tabular}

\begin{tabular}{cccccccc}
\multicolumn{2}{c}{ Grupo Solução salina } & \multicolumn{7}{c}{ CaO2 } \\
\hline ANIMAL & T0 & T1 & T2 & T3 & T4 & T5 & T6 \\
\hline $\mathbf{1}$ & 11,665 & 9,918 & 7,330 & 8,577 & 8,559 & 8,548 & 9,927 \\
$\mathbf{2}$ & 11,848 & 8,592 & 7,646 & 11,360 & 14,105 & 11,697 & 12,077 \\
$\mathbf{3}$ & 12,546 & 9,155 & 7,358 & 8,792 & 10,101 & 10,929 & 12,743 \\
$\mathbf{4}$ & 13,372 & 9,278 & 6,944 & 8,276 & 8,304 & 9,684 & 10,540 \\
$\mathbf{5}$ & 12,730 & 8,960 & 6,857 & 9,773 & 9,979 & 11,035 & 11,199 \\
$\mathbf{6}$ & 13,392 & 7,960 & 6,287 & 9,444 & 9,960 & 11,796 & 12,011 \\
$\mathbf{7}$ & 11,591 & 9,255 & 7,381 & 9,228 & 9,671 & 10,587 & 10,988 \\
\hline Média & 12,45 & 9,02 & 7,11 & 9,35 & 10,10 & 10,61 & 11,36 \\
DP & 0,77 & 0,61 & 0,45 & 1,03 & 1,91 & 1,15 & 0,98 \\
\hline
\end{tabular}




\begin{tabular}{cccccccc}
\multicolumn{2}{l}{ Grupo controle } & CvO2 & mL.dL $^{-1}$ & & & \\
\hline ANIMAL & T0 & T1 & T2 & T3 & T4 & T5 & T6 \\
\hline $\mathbf{1}$ & 11,167 & 10,160 & 9,775 & 10,491 & 11,301 & 10,936 & 10,783 \\
$\mathbf{2}$ & 8,635 & 8,430 & 8,000 & 7,824 & 9,276 & 11,501 & 10,300 \\
$\mathbf{3}$ & 9,791 & 9,755 & 10,526 & 9,795 & 10,327 & 10,670 & 10,470 \\
$\mathbf{4}$ & 8,476 & 9,555 & 9,746 & 8,694 & 7,857 & 9,828 & 11,555 \\
$\mathbf{5}$ & 8,957 & 9,615 & 8,752 & 8,621 & 9,114 & 9,906 & 9,760 \\
$\mathbf{6}$ & 12,443 & 12,178 & 12,958 & 12,882 & 12,592 & 13,265 & 13,196 \\
$\mathbf{7}$ & 10,430 & 10,211 & 10,483 & 10,243 & 10,528 & 11,017 & 10,663 \\
\hline Média & 9,99 & 9,99 & 10,03 & 9,79 & 10,14 & 11,02 & 10,96 \\
DP & 1,46 & 1,13 & 1,58 & 1,67 & 1,56 & 1,16 & 1,12 \\
\hline
\end{tabular}

\begin{tabular}{|c|c|c|c|c|c|c|c|}
\hline Grupo HE & & $\mathrm{vO2}$ & & & & & \\
\hline ANIMAL & T0 & T1 & T2 & T3 & T4 & T5 & T6 \\
\hline 1 & 10,155 & 6,538 & 5,476 & 5,021 & 6,928 & 7,573 & 9,077 \\
\hline 2 & 10,079 & 6,930 & 4,986 & 5,473 & 5,473 & 7,873 & 8,658 \\
\hline 4 & 9,466 & 7,088 & 4,416 & 5,436 & 7,376 & 7,610 & 9,328 \\
\hline 7 & 10,425 & 6,663 & 5,259 & 7,368 & 8,260 & 8,422 & 10,035 \\
\hline 8 & 10,570 & 7,106 & 5,397 & 6,578 & 6,837 & 8,678 & 9,663 \\
\hline 9 & 9,681 & 6,713 & 5,146 & 6,049 & 7,387 & 9,084 & 9,089 \\
\hline 10 & 10,644 & 6,252 & 4,915 & 5,199 & 5,250 & 7,248 & 8,597 \\
\hline Média & 10,15 & 6,76 & 5,08 & 5,88 & 6,79 & 8,07 & 9,21 \\
\hline DP & 0,45 & 0,31 & 0,36 & 0,85 & 1,08 & 0,67 & 0,52 \\
\hline
\end{tabular}

\begin{tabular}{cccccccc}
\multicolumn{2}{l}{ Grupo Solução salina } & \multicolumn{7}{c}{ CvO2 } \\
\hline ANIMAL & T0 & T1 & T2 & T3 & T4 & T5 & T6 \\
\hline $\mathbf{1}$ & 8,993 & 7,260 & 5,051 & 5,431 & 5,171 & 5,765 & 6,901 \\
$\mathbf{2}$ & 9,365 & 6,376 & 5,255 & 7,375 & 9,664 & 8,424 & 8,660 \\
$\mathbf{3}$ & 9,822 & 7,195 & 5,593 & 6,944 & 7,703 & 9,042 & 10,543 \\
$\mathbf{4}$ & 11,467 & 7,713 & 5,409 & 6,088 & 5,931 & 7,727 & 8,595 \\
$\mathbf{5}$ & 9,966 & 6,785 & 4,796 & 6,815 & 6,936 & 8,910 & 8,711 \\
$\mathbf{6}$ & 11,070 & 6,388 & 4,758 & 7,033 & 7,391 & 9,776 & 9,869 \\
$\mathbf{7}$ & 7,975 & 6,824 & 5,414 & 6,526 & 7,075 & 8,383 & 8,503 \\
\hline Média & 9,81 & 6,93 & 5,18 & 6,60 & 7,12 & 8,29 & 8,83 \\
DP & 1,20 & 0,49 & 0,32 & 0,66 & 1,42 & 1,28 & 1,15 \\
\hline
\end{tabular}




\begin{tabular}{cccccccc}
\multicolumn{2}{l}{ Grupo controle } & C(a-v)O2 & \multicolumn{7}{l}{} \\
\hline ANIMAL & T0 & T1 & T2 & T3 & T4 & T5 & T6 \\
\hline $\mathbf{1}$ & 2,824 & 2,930 & 3,287 & 3,482 & 3,886 & 4,304 & 4,522 \\
$\mathbf{2}$ & 3,840 & 3,900 & 4,156 & 4,343 & 3,946 & 3,499 & 3,600 \\
$\mathbf{3}$ & 2,336 & 2,399 & 2,612 & 2,430 & 2,901 & 3,067 & 3,080 \\
$\mathbf{4}$ & 2,895 & 2,660 & 2,880 & 2,687 & 2,590 & 2,913 & 2,585 \\
$\mathbf{5}$ & 2,875 & 3,157 & 3,475 & 3,588 & 3,587 & 3,192 & 3,259 \\
$\mathbf{6}$ & 2,585 & 2,810 & 2,963 & 3,036 & 3,371 & 3,119 & 3,230 \\
$\mathbf{7}$ & 2,313 & 2,927 & 2,860 & 3,011 & 2,674 & 2,673 & 3,048 \\
\hline Média & 2,81 & 2,97 & 3,18 & 3,23 & 3,28 & 3,25 & 3,33 \\
DP & 0,52 & 0,47 & 0,52 & 0,64 & 0,56 & 0,53 & 0,61 \\
\hline
\end{tabular}

\begin{tabular}{cccccccc} 
Grupo HES & \multicolumn{7}{c}{ C(a-v)O2 } \\
\hline ANIMAL & T0 & T1 & T2 & T3 & T4 & T5 & T6 \\
\hline $\mathbf{1}$ & 2,154 & 1,946 & 1,663 & 1,530 & 1,977 & 2,339 & 2,017 \\
$\mathbf{2}$ & 2,823 & 2,246 & 2,394 & 2,762 & 2,719 & 2,696 & 2,798 \\
$\mathbf{4}$ & 2,428 & 2,054 & 2,009 & 2,367 & 2,801 & 2,603 & 2,627 \\
$\mathbf{7}$ & 2,464 & 2,175 & 2,002 & 2,719 & 2,426 & 2,233 & 2,488 \\
$\mathbf{8}$ & 2,840 & 1,669 & 1,575 & 1,685 & 2,051 & 1,984 & 2,238 \\
$\mathbf{9}$ & 3,416 & 2,775 & 2,153 & 2,589 & 3,054 & 2,703 & 3,092 \\
$\mathbf{1 0}$ & 2,697 & 2,433 & 2,496 & 2,593 & 2,553 & 2,273 & 2,293 \\
\hline Média & 2,69 & 2,19 & 2,04 & 2,32 & 2,51 & 2,40 & 2,51 \\
DP & 0,40 & 0,35 & 0,34 & 0,50 & 0,39 & 0,27 & 0,37 \\
\hline
\end{tabular}

\begin{tabular}{cccccccc}
\multicolumn{2}{c}{ Grupo Solução salina } & \multicolumn{7}{c}{ C(a-v)O2 } \\
\hline ANIMAL & T0 & T1 & T2 & T3 & T4 & T5 & T6 \\
\hline $\mathbf{1}$ & 2,672 & 2,659 & 2,279 & 3,146 & 3,388 & 2,784 & 3,026 \\
$\mathbf{2}$ & 2,482 & 2,215 & 2,390 & 3,985 & 4,441 & 3,273 & 3,417 \\
$\mathbf{3}$ & 2,724 & 1,960 & 1,765 & 1,848 & 2,397 & 1,887 & 2,200 \\
$\mathbf{4}$ & 1,905 & 1,565 & 1,535 & 2,188 & 2,373 & 1,957 & 1,946 \\
$\mathbf{5}$ & 2,764 & 2,176 & 2,062 & 2,958 & 3,043 & 2,124 & 2,488 \\
$\mathbf{6}$ & 2,322 & 1,572 & 1,528 & 2,412 & 2,569 & 2,020 & 2,142 \\
$\mathbf{7}$ & 3,615 & 2,432 & 1,967 & 2,701 & 2,596 & 2,204 & 2,485 \\
\hline Média & 2,64 & 2,08 & 1,93 & 2,75 & 2,97 & 2,32 & 2,53 \\
DP & 0,52 & 0,41 & 0,34 & 0,70 & 0,74 & 0,51 & 0,52 \\
\hline
\end{tabular}




\begin{tabular}{cccccccc}
\multicolumn{2}{c}{ Grupo controle } & IDO2 & mL. $\mathbf{m i n}^{-\mathbf{1}} \cdot \mathbf{m}^{-\mathbf{2}}$ \\
\hline ANIMAL & T0 & T1 & T2 & T3 & T4 & T5 & T6 \\
\hline $\mathbf{1}$ & 685,55 & 597,30 & 548,60 & 614,79 & 577,08 & 533,42 & 566,27 \\
$\mathbf{2}$ & & & & & & & \\
$\mathbf{3}$ & 582,08 & 559,09 & 551,79 & 562,38 & 555,56 & 563,22 & 580,30 \\
$\mathbf{4}$ & 625,37 & 586,32 & 681,81 & 603,20 & 574,56 & 649,77 & 735,27 \\
$\mathbf{5}$ & 638,96 & 613,06 & 599,14 & 586,03 & 596,97 & 681,09 & 677,02 \\
$\mathbf{6}$ & 736,39 & 689,42 & 811,98 & 732,26 & 702,38 & 720,90 & 722,75 \\
$\mathbf{7}$ & 662,61 & 538,66 & 573,76 & 636,17 & 726,09 & 670,81 & 644,39 \\
\hline Média & 655,2 & 597,3 & 627,8 & 622,5 & 622,1 & 636,5 & 654,3 \\
DP & 53,0 & 52,4 & 102,6 & 59,3 & 72,9 & 72,7 & 70,8 \\
\hline
\end{tabular}

\begin{tabular}{cccccccc} 
Grupo HES & \multicolumn{1}{c}{ IDO2 } & \multicolumn{7}{l}{ T3 } & T4 & T5 & T6 \\
\hline ANIMAL & T0 & T1 & T2 & T3 & 678,92 \\
\hline $\mathbf{1}$ & 713,94 & 653,25 & 678,18 & 504,48 & 650,05 & 654,21 & $820,96,49$ \\
$\mathbf{2}$ & 735,39 & 605,61 & 538,78 & 452,93 & 532,48 & 718,68 & 664,49 \\
$\mathbf{4}$ & 642,24 & 612,52 & 501,15 & 476,00 & 539,42 & 582,16 & 609,71 \\
$\mathbf{7}$ & 824,88 & 689,35 & 675,23 & 766,62 & 758,71 & 681,95 & 851,53 \\
$\mathbf{8}$ & 724,16 & 631,80 & 634,43 & 619,76 & 604,40 & 788,97 & 749,75 \\
$\mathbf{9}$ & 589,39 & 692,64 & 627,70 & 578,75 & 689,12 & 872,22 & 718,69 \\
$\mathbf{1 0}$ & 720,41 & 590,56 & 659,58 & 607,77 & 561,85 & 790,26 & 827,68 \\
\hline Média & 707,2 & 639,4 & 616,4 & 572,3 & 619,4 & 726,9 & 749,0 \\
DP & 74,4 & 40,5 & 69,4 & 107,6 & 84,4 & 97,7 & 90,7 \\
\hline
\end{tabular}

Grupo Solução salina

IDO2

\begin{tabular}{cccccccc}
\hline ANIMAL & T0 & T1 & T2 & T3 & T4 & T5 & T6 \\
\hline $\mathbf{1}$ & 618,25 & 525,67 & 586,43 & 454,61 & 470,75 & 538,52 & 714,72 \\
$\mathbf{2}$ & 639,77 & 463,94 & 405,22 & 488,47 & 620,60 & 526,38 & 519,33 \\
$\mathbf{3}$ & 702,58 & 732,41 & 551,86 & 650,57 & 646,45 & 754,07 & 866,53 \\
$\mathbf{4}$ & 842,45 & 807,18 & 597,16 & 496,54 & 489,94 & 745,64 & 779,99 \\
$\mathbf{5}$ & 611,04 & 672,01 & 486,88 & 664,54 & 518,91 & 761,40 & 716,72 \\
$\mathbf{6}$ & 736,57 & 644,75 & 471,52 & 528,88 & 478,08 & 825,73 & 876,84 \\
$\mathbf{7}$ & 498,39 & 536,81 & 509,27 & 572,12 & 686,64 & 815,23 & 703,21 \\
\hline Média & 691,78 & 641,00 & 516,51 & 547,27 & 537,45 & 691,96 & 745,69 \\
DP & 88,83 & 127,75 & 74,74 & 88,75 & 76,64 & 126,82 & 131,16 \\
\hline
\end{tabular}




\begin{tabular}{cccccccc}
\multicolumn{2}{l}{ Grupo controle } & IVO2 & \multicolumn{7}{c}{${\text { mL. } \mathbf{m i n}^{-\mathbf{1}} \cdot \mathbf{m}^{-\mathbf{2}}}^{\text {ANIMAL }}$} & T0 & T1 & T2 & T3 & T4 & T5 & T6 \\
\hline $\mathbf{1}$ & 138,36 & 127,80 & 138,04 & 153,20 & 147,66 & 150,65 & 167,31 \\
$\mathbf{2}$ & & & & & & & \\
$\mathbf{3}$ & 112,12 & 110,37 & 109,70 & 111,79 & 121,83 & 125,75 & 133,50 \\
$\mathbf{4}$ & 159,22 & 127,70 & 155,54 & 142,40 & 142,43 & 148,56 & 134,40 \\
$\mathbf{5}$ & 155,27 & 151,53 & 170,30 & 172,24 & 168,61 & 165,97 & 169,49 \\
$\mathbf{6}$ & 126,66 & 129,25 & 151,14 & 139,68 & 148,33 & 137,25 & 142,12 \\
$\mathbf{7}$ & 120,26 & 120,00 & 122,98 & 144,51 & 147,07 & 130,97 & 143,25 \\
\hline Média & 135,3 & 127,8 & 141,3 & 144,0 & 146,0 & 143,2 & 151,3 \\
DP & 19,1 & 15,2 & 22,3 & 19,7 & 14,9 & 14,8 & 16,0 \\
\hline
\end{tabular}

\begin{tabular}{cccccccc} 
Grupo HES & \multicolumn{1}{c}{ IVO2 } & \multicolumn{7}{l}{} \\
\hline ANIMAL & T0 & T1 & T2 & T3 & T4 & T5 & T6 \\
\hline $\mathbf{1}$ & 124,95 & 149,82 & 158,00 & 117,84 & 144,34 & 154,37 & 149,23 \\
$\mathbf{2}$ & 160,91 & 148,22 & 174,78 & 151,89 & 176,75 & 183,31 & 162,30 \\
$\mathbf{4}$ & 131,09 & 137,60 & 156,68 & 144,41 & 148,47 & 148,39 & 133,99 \\
$\mathbf{7}$ & 157,67 & 169,63 & 186,17 & 206,63 & 172,27 & 142,94 & 169,16 \\
$\mathbf{8}$ & 153,38 & 120,13 & 143,30 & 126,40 & 139,48 & 146,78 & 140,98 \\
$\mathbf{9}$ & 153,74 & 202,56 & 185,18 & 173,44 & 201,55 & 200,00 & 182,44 \\
$\mathbf{1 0}$ & 145,63 & 165,41 & 222,14 & 202,24 & 183,83 & 188,64 & 174,29 \\
\hline Média & 146,8 & 156,2 & 175,2 & 160,4 & 166,7 & 166,3 & 158,9 \\
DP & 13,8 & 26,3 & 26,1 & 35,0 & 23,1 & 23,5 & 18,0 \\
\hline
\end{tabular}

Grupo Solução salina

IVO2

\begin{tabular}{cccccccc}
\hline ANIMAL & T0 & T1 & T2 & T3 & T4 & T5 & T6 \\
\hline $\mathbf{1}$ & 141,63 & 140,90 & 182,34 & 166,75 & 186,33 & 175,36 & 217,84 \\
$\mathbf{2}$ & 134,04 & 119,63 & 126,68 & 171,35 & 195,40 & 147,29 & 146,94 \\
$\mathbf{3}$ & 152,53 & 156,79 & 132,40 & 136,73 & 153,42 & 130,19 & 149,60 \\
$\mathbf{4}$ & 120,03 & 136,19 & 132,01 & 131,26 & 140,00 & 150,66 & 143,98 \\
$\mathbf{5}$ & 132,67 & 163,16 & 146,38 & 201,15 & 158,25 & 146,59 & 159,20 \\
$\mathbf{6}$ & 127,72 & 127,34 & 114,63 & 135,04 & 123,33 & 141,42 & 156,38 \\
$\mathbf{7}$ & 155,45 & 141,04 & 135,72 & 167,48 & 184,34 & 169,71 & 159,04 \\
\hline Média & 134,8 & 140,7 & 139,1 & 157,0 & 159,5 & 148,6 & 162,3 \\
DP & 11,3 & 16,8 & 23,5 & 27,6 & 27,3 & 14,9 & 27,8 \\
\hline
\end{tabular}




\begin{tabular}{cccccccc}
\multicolumn{6}{l}{ Grupo controle } & TEO2 & $\%$ \\
\hline ANIMAL & T0 & T1 & T2 & T3 & T4 & T5 & T6 \\
\hline $\mathbf{1}$ & 20,182 & 22,000 & 25,162 & 24,919 & 25,587 & 28,243 & 29,547 \\
$\mathbf{2}$ & 20,000 & 22,000 & 23,000 & 23,000 & 24,000 & 23,328 & 24,000 \\
$\mathbf{3}$ & 19,261 & 19,741 & 19,881 & 19,878 & 21,929 & 22,328 & 23,000 \\
$\mathbf{4}$ & 25,460 & 21,780 & 22,813 & 23,607 & 24,790 & 22,863 & 18,278 \\
$\mathbf{5}$ & 24,301 & 24,718 & 28,424 & 29,390 & 28,243 & 24,368 & 25,034 \\
$\mathbf{6}$ & 17,201 & 18,748 & 18,613 & 19,075 & 21,119 & 19,039 & 19,665 \\
$\mathbf{7}$ & 18,150 & 22,277 & 21,435 & 22,716 & 20,256 & 19,524 & 22,230 \\
\hline Média & 20,7 & 21,6 & 22,8 & 23,2 & 23,7 & 22,8 & 23,1 \\
DP & 3,1 & 1,9 & 3,3 & 3,4 & 2,8 & 3,1 & 3,7 \\
\hline
\end{tabular}

\begin{tabular}{|c|c|c|c|c|c|c|c|}
\hline Grupo HE & & 02 & & & & & \\
\hline ANIMAL & T0 & T1 & T2 & T3 & T4 & T5 & T6 \\
\hline 1 & 17,502 & 22,934 & 23,297 & 23,359 & 22,204 & 23,596 & 18,179 \\
\hline 2 & 21,881 & 24,475 & 32,441 & 33,536 & 33,194 & 25,506 & 24,424 \\
\hline 4 & 20,411 & 22,464 & 31,264 & 30,338 & 27,525 & 25,490 & 21,976 \\
\hline 7 & 19,115 & 24,607 & 27,571 & 26,953 & 22,706 & 20,961 & 19,866 \\
\hline 8 & 21,180 & 19,014 & 22,587 & 20,394 & 23,077 & 18,604 & 18,803 \\
\hline 9 & 26,084 & 29,245 & 29,502 & 29,968 & 29,248 & 22,929 & 25,385 \\
\hline 10 & 20,216 & 28,009 & 33,679 & 33,276 & 32,718 & 23,871 & 21,058 \\
\hline Média & 20,9 & 24,4 & 28,6 & 28,3 & 27,2 & 23,0 & 21,4 \\
\hline DP & 2,7 & 3,5 & 4,4 & 5,0 & 4,7 & 2,5 & 2,7 \\
\hline
\end{tabular}

Grupo Solução salina TEO2

\begin{tabular}{cccccccc}
\hline ANIMAL & T0 & T1 & T2 & T3 & T4 & T5 & T6 \\
\hline $\mathbf{1}$ & 22,908 & 26,805 & 31,093 & 36,679 & 39,582 & 32,563 & 30,479 \\
$\mathbf{2}$ & 20,952 & 25,784 & 31,263 & 35,079 & 31,485 & 27,982 & 28,295 \\
$\mathbf{3}$ & 21,711 & 21,408 & 23,992 & 21,016 & 23,733 & 17,264 & 17,265 \\
$\mathbf{4}$ & 14,248 & 16,873 & 22,107 & 26,435 & 28,575 & 20,206 & 18,460 \\
$\mathbf{5}$ & 21,713 & 24,280 & 30,065 & 30,269 & 30,497 & 19,252 & 22,213 \\
$\mathbf{6}$ & 17,340 & 19,750 & 24,311 & 25,534 & 25,797 & 17,127 & 17,835 \\
$\mathbf{7}$ & 31,190 & 26,274 & 26,650 & 29,273 & 26,846 & 20,818 & 22,616 \\
\hline Média & 21,4 & 23,0 & 27,1 & 29,2 & 29,5 & 22,2 & 22,5 \\
DP & 5,3 & 3,8 & 3,8 & 5,5 & 5,2 & 5,9 & 5,2 \\
\hline
\end{tabular}




\begin{tabular}{cccccccc}
\multicolumn{2}{l}{ Grupo controle } & \multicolumn{2}{l}{ FE ecocardiograma } & \multicolumn{2}{c}{$\%$} \\
\hline ANIMAL & T0 & T1 & T2 & T3 & T4 & T5 & T6 \\
\hline $\mathbf{1}$ & 65,16 & 62,7 & 58,03 & 62,3 & 61,76 & 57,86 & 65,36 \\
$\mathbf{2}$ & 72,2 & 70,1 & 66,4 & 63,56 & 68,36 & 70 & 67,3 \\
$\mathbf{3}$ & 59,46 & 57,9 & 61,23 & 69,63 & 62,3 & 67 & 63 \\
$\mathbf{4}$ & 66,93 & 66,6 & 69,4 & 63,7 & 65,67 & 69,9 & 62,73 \\
$\mathbf{5}$ & 72,23 & 70,16 & 69,33 & 65,86 & 68 & 65,06 & 66,96 \\
$\mathbf{6}$ & 67,26 & 72,2 & 70,7 & 69,1 & 68,2 & 68,9 & 70,76 \\
$\mathbf{7}$ & 66,83 & 65,7 & 64,46 & 62,7 & 60,13 & 60,2 & 63,26 \\
\hline Média & 67,15 & 66,48 & 65,65 & 65,26 & 64,92 & 65,56 & 65,62 \\
DP & 4,37 & 4,96 & 4,70 & 3,02 & 3,47 & 4,83 & 2,94 \\
\hline
\end{tabular}

\begin{tabular}{cccccccc} 
Grupo HES & \multicolumn{7}{c}{ FE ecocardiograma } \\
\hline ANIMAL & T0 & T1 & T2 & T3 & T4 & T5 & T6 \\
\hline $\mathbf{1}$ & 76,36 & 81,83 & 79,7 & 77,63 & 72,33 & 73,43 & 75,1 \\
$\mathbf{2}$ & 71,76 & 75,86 & 70,06 & 70,36 & 74,46 & 72,7 & 70,3 \\
$\mathbf{3}$ & 70,73 & 71,87 & 69,8 & 71,4 & 71,37 & 71,87 & 69 \\
$\mathbf{4}$ & 71,3 & 71,36 & 72,1 & 62,7 & 61,7 & 64,23 & 62,7 \\
$\mathbf{5}$ & 72,4 & 73,83 & 72,8 & 74,53 & 71,06 & 70,56 & 68,33 \\
$\mathbf{6}$ & 66,6 & 68,23 & 72,16 & 72,1 & 70,4 & 61,8 & 62,96 \\
$\mathbf{7}$ & 67,23 & 67 & 67,86 & 68,13 & 70,03 & 67,23 & 68,93 \\
\hline Média & 70,91 & 72,85 & 70,80 & 70,98 & 70,19 & 68,83 & 68,19 \\
DP & 3,29 & 4,99 & 1,88 & 4,75 & 4,02 & 4,50 & 4,30 \\
\hline
\end{tabular}

Grupo Solução salina

FE ecocardiograma

\begin{tabular}{cccccccc}
\hline ANIMAL & T0 & T1 & T2 & T3 & T4 & T5 & T6 \\
\hline $\mathbf{1}$ & 68,7 & 75,23 & 75,23 & 75,83 & 71,96 & 71,8 & 75,63 \\
$\mathbf{2}$ & 68,6 & 62,5 & 61,86 & 62,56 & 63,93 & 58,83 & 64,3 \\
$\mathbf{3}$ & 68,8 & 73,76 & 73,03 & 66,93 & 48,3 & 65,46 & 60,6 \\
$\mathbf{4}$ & 67,3 & 76,83 & 74,96 & 73,1 & 71,1 & 74,3 & 73,33 \\
$\mathbf{5}$ & 68,46 & 63,23 & 69,03 & 67,56 & 63,36 & 73,76 & 74,93 \\
$\mathbf{6}$ & 70,56 & 71,9 & 65,45 & 68,06 & 73,7 & 67,56 & 69,66 \\
$\mathbf{7}$ & 65,2 & 73,4 & 68,76 & 66,5 & 62,26 & 69,3 & 70,3 \\
\hline Média & 68,76 & 70,86 & 70,14 & 68,98 & 65,31 & 68,66 & 70,17 \\
DP & 2,13 & 5,34 & 4,75 & 4,20 & 8,10 & 5,02 & 5,28 \\
\hline
\end{tabular}




\begin{tabular}{cccccccc}
\multicolumn{2}{l}{ Grupo controle } & \multicolumn{2}{l}{ EDV ecocardiograma } & \multicolumn{2}{c}{$\mathbf{~}$} \\
\hline ANIMAL & T0 & T1 & T2 & T3 & T4 & T5 & T6 \\
\hline $\mathbf{1}$ & 44,3 & 45,3 & 48,3 & 59,8 & 55,86 & 51,1 & 55,16 \\
$\mathbf{2}$ & 41,23 & 40,2 & 42,93 & 42,4 & 39,7 & 31,8 & 34,3 \\
$\mathbf{3}$ & 48,03 & 47,5 & 48,33 & 42,66 & 39,53 & 44,9 & 43,9 \\
$\mathbf{4}$ & 52,8 & 44,16 & 36,83 & 35 & 27,57 & 27,7 & 31,13 \\
$\mathbf{5}$ & 32,56 & 30 & 29,43 & 30,66 & 28,76 & 23,3 & 29,06 \\
$\mathbf{6}$ & 34,2 & 38,3 & 29,63 & 36,13 & 35,06 & 28,63 & 34,3 \\
$\mathbf{7}$ & 44 & 38,83 & 41,83 & 36,1 & 34,56 & 34,36 & 34,16 \\
\hline Média & 42,45 & 40,61 & 39,61 & 40,39 & 37,29 & 34,54 & 37,43 \\
DP & 7,20 & 5,82 & 7,94 & 9,54 & 9,45 & 9,98 & 9,09 \\
\hline
\end{tabular}

\begin{tabular}{cccccccc} 
Grupo HES & \multicolumn{7}{c}{ EDV ecocardiograma } \\
\hline ANIMAL & T0 & T1 & T2 & T3 & T4 & T5 & T6 \\
\hline $\mathbf{1}$ & 27,96 & 30,66 & 33,7 & 35,23 & 36,2 & 50,13 & 43,23 \\
$\mathbf{2}$ & 37,26 & 36,26 & 42,86 & 32,13 & 38,16 & 54,6 & 45 \\
$\mathbf{3}$ & 45,87 & 47,73 & 47,37 & 36,23 & 40,93 & 40,23 & 36,6 \\
$\mathbf{4}$ & 30,16 & 31,66 & 35,1 & 23,9 & 18,33 & 29,63 & 22,33 \\
$\mathbf{5}$ & 39,5 & 40,96 & 49,16 & 45,33 & 38,23 & 46,43 & 44,4 \\
$\mathbf{6}$ & 32,8 & 33,73 & 39,66 & 34,63 & 30,5 & 37,53 & 36,63 \\
$\mathbf{7}$ & 58,73 & 58,26 & 61,26 & 63,03 & 65,73 & 60,4 & 58,43 \\
\hline Média & 38,90 & 39,89 & 45,90 & 38,64 & 38,30 & 45,56 & 40,95 \\
DP & 10,63 & 10,03 & 9,09 & 12,47 & 14,28 & 10,57 & 10,98 \\
\hline
\end{tabular}

\begin{tabular}{cccccccc}
\multicolumn{2}{c}{ Grupo Solução salina } & \multicolumn{7}{c}{ EDV ecocardiograma } \\
\hline \multicolumn{1}{l}{ ANIMAL } & T0 & T1 & T2 & T3 & T4 & T5 & T6 \\
\hline $\mathbf{1}$ & 34,5 & 35,3 & 34,2 & 33,8 & 28,23 & 40,36 & 41,5 \\
$\mathbf{2}$ & 38,06 & 39,53 & 34,3 & 30,53 & 33,23 & 35,43 & 33,83 \\
$\mathbf{3}$ & & & & & & & \\
$\mathbf{4}$ & 45,93 & 48,96 & 33,46 & 27,56 & 30,23 & 48,56 & 40,26 \\
$\mathbf{5}$ & 27,4 & 17,3 & 17,5 & 17,33 & 13 & 32,8 & 33,4 \\
$\mathbf{6}$ & 51,7 & 52,8 & 32,8 & 32,43 & 39,33 & 48,1 & 50,76 \\
$\mathbf{7}$ & 38,53 & 39,4 & 30,33 & 26,06 & 15,56 & 36,86 & 32,63 \\
\hline Média & 39,35 & 38,88 & 30,43 & 27,95 & 26,60 & 40,35 & 39,95 \\
DP & 8,54 & 12,45 & 6,50 & 5,96 & 10,28 & 6,65 & 7,07 \\
\hline
\end{tabular}




\begin{tabular}{cccccccc}
\multicolumn{2}{l}{ Grupo controle } & isoflurano & exp \% & & & \\
\hline ANIMAL & T0 & T1 & T2 & T3 & T4 & T5 & T6 \\
\hline $\mathbf{1}$ & & & & & & & \\
$\mathbf{2}$ & 0,8 & 1,3 & 1 & 1 & 1,1 & 1,3 & 1,2 \\
$\mathbf{3}$ & & & & & & & \\
$\mathbf{4}$ & 1,2 & 0,9 & 1 & 1 & 1 & 1,1 & 1,1 \\
$\mathbf{5}$ & 1,3 & 1,4 & 1,3 & 1,3 & 1,3 & 1,3 & 1,4 \\
$\mathbf{6}$ & 1,5 & 1,4 & 1,6 & 1,7 & 1,7 & 1,6 & 1,6 \\
$\mathbf{7}$ & 1,4 & 1,5 & 1,5 & 1,7 & 1,7 & 1,7 & 1,7 \\
\hline Média & 1,2 & 1,3 & 1,3 & 1,3 & 1,4 & 1,4 & 1,5 \\
DP & 0,2 & 0,3 & 0,3 & 0,4 & 0,3 & 0,3 & 0,3 \\
\hline
\end{tabular}

\begin{tabular}{cccccccc} 
Grupo HES & & isoflurano & exp \% & & & & \\
\hline ANIMAL & T0 & T1 & T2 & T3 & T4 & T5 & T6 \\
\hline $\mathbf{1}$ & 1,1 & 1,1 & 1,1 & 1,1 & 1,1 & 1,2 & 1,2 \\
$\mathbf{2}$ & 1 & 1 & 1 & 1,1 & 1,1 & 1 & 1,1 \\
$\mathbf{3}$ & 1,3 & 1,3 & 1,4 & 1,4 & 1,5 & 1,5 & 1,5 \\
$\mathbf{4}$ & 1,3 & 1,4 & 1,5 & 1,5 & 1,4 & 1,5 & 1,5 \\
$\mathbf{5}$ & 1,5 & 1,7 & 1,4 & 1,5 & 1,6 & 1,6 & 1,6 \\
$\mathbf{6}$ & 1,4 & 1,5 & 1,5 & 1,5 & 1,4 & 1,4 & 1,2 \\
$\mathbf{7}$ & 1,4 & 1,2 & 1,6 & 1,5 & 1,4 & 1,3 & 1,4 \\
\hline Média & 1,3 & 1,3 & 1,4 & 1,4 & 1,4 & 1,4 & 1,4 \\
DP & 0,2 & 0,2 & 0,2 & 0,2 & 0,2 & 0,2 & 0,2 \\
\hline
\end{tabular}

\begin{tabular}{|c|c|c|c|c|c|c|c|}
\hline Grupo S & lo sa & & flura & $\%$ & & & \\
\hline ANIMAL & T0 & T1 & T2 & T3 & T4 & T5 & T6 \\
\hline 1 & 1,1 & 1,1 & 1,2 & 1,2 & 1,2 & 1,2 & 1,2 \\
\hline 2 & 1,2 & 1,3 & 1,3 & 1,3 & 1,4 & 1,4 & 1,4 \\
\hline 3 & 1,3 & 1,4 & 1,4 & 1,4 & 1,4 & 1,4 & 1,5 \\
\hline 4 & 1,4 & 1,4 & 1,4 & 1,5 & 1,5 & 1,5 & 1,4 \\
\hline 5 & 1,6 & 1,6 & 1,7 & 1,3 & 1,2 & 1,3 & 1,3 \\
\hline 6 & 1,3 & 1,3 & 1,4 & 1,4 & 1,4 & 1,4 & 1,4 \\
\hline 7 & 1,6 & 1,5 & 1,6 & 1,4 & 1,4 & 1,4 & 1,4 \\
\hline Média & 1,4 & 1,4 & 1,4 & 1,4 & 1,4 & 1,4 & 1,4 \\
\hline DP & 0,2 & 0,2 & 0,2 & 0,1 & 0,1 & 0,1 & 0,1 \\
\hline
\end{tabular}




\section{REFERÊNCIAS BIBLIOGRÁFICAS}




\section{REFERÊNCIAS BIBLIOGRÁFICAS}

1. Grocott MP, Mythen MG, Gan TJ. Perioperative fluid management and clinical outcomes in adults. Anesth Analg. 2005 Apr;100(4):1093-106.

2. Michard F, Teboul JL. Predicting fluid responsiveness in ICU patients: a critical analysis of the evidence. Chest. 2002 Jun;121(6):2000-8.

3. Michard F, Boussat S, Chemla D, Anguel N, Mercat A, Lecarpentier $\mathrm{Y}$, Richard C, Pinsky MR, Teboul JL. Relation between respiratory changes in arterial pulse pressure and fluid responsiveness in septic patients with acute circulatory failure. Am J Respir Crit Care Med. 2000 Jul;162(1):134-8.

4. Carlos RV, Bittar CS, Lopes MR, Auler Jr. JOC. Variação da pressão sistólica como método diagnóstico da hipovolemia durante anestesia para cirurgia cardíaca. Revista brasileira de Anestesiologia. 2005;55(1):3-18.

5. Solus-Biguenet $\mathrm{H}$, Fleyfel M, Tavernier B, Kipnis E, Onimus J, Robin E, Lebuffe G, Decoene C, Pruvot FR, Vallet B. Non-invasive prediction of fluid responsiveness during major hepatic surgery. $\mathrm{Br} \mathrm{J}$ Anaesth. 2006 Dec;97(6):808-16.

6. Jamnicki $M K R$, Vanderlinden $P$, Zaugg $M$, Sphan $D R$. Acute normovolemic hemodilution: Physiology, limitations, and clinical use. Journal of Cardiothoracic and Vascular Anesthesia. 2003;17:747-54.

7. Weber RS, Jabbour N, Martin RC, 2nd. Anemia and transfusions in patients undergoing surgery for cancer. Ann Surg Oncol. 2008 Jan;15(1):34-45.

8. Hebert PC, Wells G, Blajchman MA, Marshall J, Martin C, Pagliarello G, Tweeddale M, Schweitzer I, Yetisir E. A multicenter, randomized, controlled clinical trial of transfusion requirements in critical care. Transfusion Requirements in Critical Care Investigators, Canadian Critical Care Trials Group. N Engl J Med. 1999 Feb 11;340(6):409-17.

9. Fraga Ade O, Fantoni DT, Otsuki DA, Pasqualucci CA, Abduch MC, Auler Jr. JOC. Evidence for myocardial defects under extreme acute normovolemic 
hemodilution with hydroxyethyl starch and lactated ringer's solution. Shock. 2005 Oct;24(4):388-95.

10. Margarido CB, Margarido NF, Otsuki DA, Fantoni DT, Marumo CK, Kitahara FR, Magalhaes AA, Pasqualucci CA, Auler Jr. JOC, Pulmonary function is better preserved in pigs when acute normovolemic hemodilution is achieved with hydroxyethyl starch versus lactated Ringer's solution. Shock. 2007 Apr;27(4):390-6.

11. Otsuki DA, Fantoni DT, Margarido CB, Marumo CK, Intelizano T, Pasqualucci CA, Costa Auler Jr. JOC, Hydroxyethyl starch is superior to lactated Ringer as a replacement fluid in a pig model of acute normovolaemic haemodilution. Br J Anaesth. 2007 Jan;98(1):29-37.

12. Fantoni DT, Otsuki DA, Ambrosio AM, Tamura EY, Auler Jr. JOC A comparative evaluation of inhaled halothane, isoflurane, and sevoflurane during acute normovolemic hemodilution in dogs. Anesth Analg. 2005 Apr;100(4):1014-9.

13. Hollenberg S M AT, Annane D, Astiz M E, Chalfin D B, Dasta J F, Heard SO, Martin C, Napolitano LM, Susla G M, Tortaro R, Vcent J L, ZannotiCavazzon S. Practice Parameters for hemodynamic support of sepsis in adult patients: 2004 update. Critical Care Medicine. 2004;32:1928-48.

14. Hofer CK, Muller SM, Furrer L, Klaghofer R, Genoni M, Zollinger A. Stroke volume and pulse pressure variation for prediction of fluid responsiveness in patients undergoing off-pump coronary artery bypass grafting. Chest. 2005 Aug;128(2):848-54.

15. Kern J W SWC. Meta-analysis of hemodynamic optimization in high-risk patients. Critical Care Medicine. 2002;30:1686-92.

16. Reuter DA, Goetz AE. Differentiating "volumetric preload monitoring" and assessing "fluid responsiveness". Anesth Analg. 2006 Feb;102(2):651-2; author reply 2 .

17. Pinsky MR, Teboul JL. Assessment of indices of preload and volume responsiveness. Curr Opin Crit Care. 2005 Jun;11(3):235-9. 
18. Magder S. Clinical usefulness of respiratory variations in arterial pressure. American Journal of Respiratory and Critical Care Medicine. 2004;15:151-5.

19. Perel A, Pizov R, Cotev S. Systolic blood pressure variation is a sensitive indicator of hypovolemia in ventilated dogs subjected to graded hemorrhage. Anesthesiology. 1987 Oct;67(4):498-502.

20. Nascimento P, Jr., de Paiva Filho O, de Carvalho LR, Braz JR. Early hemodynamic and renal effects of hemorrhagic shock resuscitation with lactated Ringer's solution, hydroxyethyl starch, and hypertonic saline with or without 6\% dextran-70. J Surg Res. 2006 Nov;136(1):98-105.

21. Michard F. Changes in arterial pressure during mechanical ventilation. Anesthesiology. 2005 Aug;103(2):419-28; quiz 49-5.

22. Bendjelid K, Romand JA. Fluid responsiveness in mechanically ventilated patients: a review of indices used in intensive care. Intensive Care Med. 2003 Mar;29(3):352-60.

23. Mitchell JP, Schuller D, Calandrino FS, Schuster DP. Improved outcome based on fluid management in critically ill patients requiring pulmonary artery catheterization. Am Rev Respir Dis. 1992 May;145(5):990-8.

24. Vieillard-Baron A, Chergui K, Augarde R, Prin S, Page B, Beauchet A, Jardin F. Cyclic changes in arterial pulse during respiratory support revisited by Doppler echocardiography. Am J Respir Crit Care Med. 2003 Sep 15;168(6):671-6.

25. Feissel M, Michard F, Faller JP, Teboul JL. The respiratory variation in inferior vena cava diameter as a guide to fluid therapy. Intensive Care Med. 2004 Sep;30(9):1834-7.

26. Cottis R, Magee N, Higgins DJ. Haemodynamic monitoring with pulseinduced contour cardiac output (PiCCO) in critical care. Intensive Crit Care Nurs. 2003 Oct;19(5):301-7.

27. Salukhe TV WD. Volumetric haemodynamic monitoring and continous pulse contour analysis - an untapped resource for coronary and high dependency 
care units? The British Journal of Cardiology (Acute and Interventional Cardiology). 2002;9:20-5.

28. Lichtwarck-Ascoff M ZJ, Pfeffer U J . Intrathoracic blood volume accurately reflects circulatory volume status in critically ill patients whith mechanical ventilation. Intensive Care Med. 1992;18:142-7.

29. Gross JB. Estimating allowable blood loss: corrected for dilution. Anesthesiology. 1983 Mar;58(3):277-80.

30. Shoemaker WCA, S.; Grenvik, A. Holbrook, P.R.; Thompson, W.L. . Diagnosis and treatment of the shock syndromes. Textbook of Critical Care, 2ed WB Saunders Company, Philadelphia,. 1995.:p. 85-102.

31. Rosner B. FUNDAMENTALS OF BIOSTATISTICS. $4^{\mathrm{a}}$ ed. New York: Duxbury Press 1994.

32. Watters JM, Brundage SI, Todd SR, Zautke NA, Stefater JA, Lam JC, Muller $P J$, Malinoski D, Schreiber MA. Resuscitation with lactated ringer's does not increase inflammatory response in a Swine model of uncontrolled hemorrhagic shock. Shock. 2004 Sep;22(3):283-7.

33. Perel P, Roberts I. Colloids versus crystalloids for fluid resuscitation in critically ill patients. Cochrane database of systematic reviews (Online). 2007(4):CD000567.

34. Franz A, Braunlich P, Gamsjager T, Felfernig M, Gustorff B, KozekLangenecker SA. The effects of hydroxyethyl starches of varying molecular weights on platelet function. Anesth Analg. 2001 Jun;92(6):1402-7.

35. Gandhi SD, Weiskopf RB, Jungheinrich C, Koorn R, Miller D, Shangraw RE, Prough DS, Baus D, Bepperling F, Warltier DC. Volume replacement therapy during major orthopedic surgery using Voluven (hydroxyethyl starch 130/0.4) or hetastarch. Anesthesiology. 2007 Jun;106(6):1120-7.

36. Bendjelid K, Suter PM, Romand JA. The respiratory change in preejection period: a new method to predict fluid responsiveness. J Appl Physiol. 2004 Jan;96(1):337-42. 
37. Auler Jr. JOC, Galas F, Hajjar L, Santos L, Carvalho T, Michard F. Online monitoring of pulse pressure variation to guide fluid therapy after cardiac surgery. Anesth Analg. 2008 Apr;106(4):1201-6, table of contents.

38. Consensus Conference:Perioperative red blood cell transfusion. J Am Med Assoc. 1988;260:2700-3.

39. American Society of Anesthesiologists Task Force on Blood Component Therapy: Practice guidelines for blood component therapy: a report by the American Society of Anesthesiologists Task Force on Blood Component Therapy. Anesthesiology. 1996;84:732-47.

40. Weiskopf RB. Efficacy of acute normovolemic hemodilution assessed as a function of fraction of blood volume lost. Anesthesiology. 2001 Mar;94(3):439-46.

41. Otsuki DA. Hemodiluição normovolêmica aguda: estudo experimental comparativo utilizando amido hidroxietílico a $6 \%$ ou solução de Ringer lactato [tese] [doutorado]. São Paulo: Universidade de São Paulo; 2004.

42. Auler Jr. JOC. Haemodilution in clinical anesthesia. Minerva anestesiologica. 2001 May;67(5):355-8.

43. Van Woerkens EC, Trouwborst A, van Lanschot JJ. Profound hemodilution: what is the critical level of hemodilution at which oxygen delivery-dependent oxygen consumption starts in an anesthetized human? Anesth Analg. 1992 Nov;75(5):818-21.

44. Boldt J, Lenz M, Kumle B, Papsdorf $M$. Volume replacement strategies on intensive care units: results from a postal survey. Intensive Care Med. 1998 Feb;24(2):147-51.

45. Michard F, Teboul JL. Using heart-lung interactions to assess fluid responsiveness during mechanical ventilation. Crit Care. 2000;4(5):282-9.

46. Hansen RM, Viquerat CE, Matthay MA, Wiener-Kronish JP, DeMarco T, Bahtia S, Marks JD, Botvinick EH, Chatterjee K. Poor correlation between pulmonary arterial wedge pressure and left ventricular end-diastolic volume 
after coronary artery bypass graft surgery. Anesthesiology. 1986 Jun;64(6):764-70.

47. Fontes ML, Bellows W, Ngo L, Mangano DT. Assessment of ventricular function in critically ill patients: limitations of pulmonary artery catheterization. Institutions of the McSPI Research Group. J Cardiothorac Vasc Anesth. 1999 Oct;13(5):521-7.

48. Pinsky MR. Functional hemodynamic monitoring. Intensive Care Med. 2002 Apr;28(4):386-8.

49. Michard F, Lopes MR, Auler Jr. JOC. Pulse pressure variation: beyond the fluid management of patients with shock. Crit Care. 2007;11(3):131.

50. Bruckner UB, Kefalianakis F, Krieter H, Messmer K. Organ blood supply and tissue oxygenation after limited normovolemic hemodilution with 3\% versus 6\% Dextran-60. Infusionstherapie und Transfusionsmedizin. 1993 Aug;20(4):130-9.

51. Colantuoni A, Bertuglia S, Intaglietta M. Quantitation of rhythmic diameter changes in arterial microcirculation. The American journal of physiology. 1984 Apr;246(4 Pt 2):H508-17.

52. Tsai AG, Friesenecker B, McCarthy $M$, Sakai $H$, Intaglietta M. Plasma viscosity regulates capillary perfusion during extreme hemodilution in hamster skinfold model. The American journal of physiology. 1998 Dec;275(6 Pt 2):H2170-80.

53. Cabrales P, Tsai AG. Plasma viscosity regulates systemic and microvascular perfusion during acute extreme anemic conditions. American journal of physiology. 2006 Nov;291(5):H2445-52.

54. Tavernier B, Makhotine O, Lebuffe G, Dupont J, Scherpereel P. Systolic pressure variation as a guide to fluid therapy in patients with sepsis-induced hypotension. Anesthesiology. 1998 Dec;89(6):1313-21.

55. Paiva Filho $\mathrm{O}$ BJ, Silva FP et al. Variação da pressão sistólica como indicador precoce de hipovolemia e guia de reposição volêmica com solução hiperosmótica e hiperoncótica no cão. Rev Bras Anestesiol. 2003;53:361-76. 
56. Paiva F. Importância da variação da pressão sistólica em comparação com parâmetros hemodinâmicos e de oxigenação na avaliação da reposição volêmica com solução de pequenos e grande volumes. Estudo experimental no cão. Botucatu: UNESP. 2005.

57. Perel A. Automated assessment of fluid responsiveness in mechanically ventilated patients. Anesth Analg. 2008 Apr;106(4):1031-3. 